Cite this article: Akhlaq A, Sheikh A, Pagliari C. Defining health information exchange: scoping review of published definitions. J Innov Health Inform. 2016;23(4):684-764

http://dx.doi.org/10.14236/jhi.v23i4.838

Copyright (C) 2016 The Author(s). Published by BCS, The Chartered Institute for IT under Creative Commons license http://creativecommons.org/ licenses/by/4.0/

Author address for correspondence Dr. Ather Akhlaq Assistant Professor of eHealth, Health and Hospital Management, Institute of Business Management, Korangi Creek, Karachi, Pakistan Email: ather.akhlaq@iobm.edu.pk

Accepted November 2016

\section{Defining health information exchange: scoping review of published definitions}

\author{
Ather Akhlaq \\ Department of Health and Hospital Management, Institute of Business Management \\ Korangi Creek, Karachi, Pakistan \\ Centre for Medical Informatics, Usher Institute of Population Health Sciences and Informatics \\ Medical School, University of Edinburgh, United Kingdom

\section{Aziz Sheikh} \\ Centre for Medical Informatics, Usher Institute of Population Health Sciences and Informatics, \\ Medical School, University of Edinburgh, United Kingdom

\section{Claudia Pagliari} \\ Usher Institute of Population Health Sciences and Informatics, Medical School, University of Edinburgh \\ United Kingdom
}

\section{ABSTRACT}

Objective The term health information exchange (HIE) is often used in health informatics, yet uncertainties remain about its precise meaning. This scoping review aimed to capture and analyse existing definitions in order to map variations in the use of this term and the concepts associated with it.

Methods Systematic literature search to identify published definitions of HIE and related terms. Medline, Web of Science, Library Information Science and Technology Abstracts, EMBASE and CINAHL Plus were searched to identify relevant papers, and Google to identify grey literature. Searches were not limited by language or date of publication. Both explicit and equivalent conceptual definitions were included. Included definitions were summarised and thematically analysed in order to identify and map the underpinning constructs.

Results Our searches revealed 603 scientific articles and 5981 website links. From these, a total of 268 unique definitions of HIE were identified and extracted: 103 from scientific databases and 165 from Google. Eleven constructs emerged from the thematic analysis. Contextual factors influenced the emphasis of the definitions and the overall framing of HIE as an organisational entity that facilitates and or as a process of sharing health information between diverse stakeholders.

Conclusions HIE is a complex and evolving construct, for which multiple definitions exist. In order to encourage consensus, the authors propose this broad definition, which encompasses the key attributes of HIE described in the literature: "HIE is the electronic mobilisation of clinical and administrative information within or across organisations in a region or community and, potentially, internationally between various systems according to locally and/or nationally recognised standards while maintaining the authenticity and accuracy of the information being exchanged, enabling stakeholders to make informed decisions to enhance healthcare quality of a patient and population. HIEs are multi-stakeholder organisations that oversee the business, operational and legal issues involved in the exchange of information."

Keywords: eHealth, health informatics, health information exchange, scoping review 


\section{INTRODUCTION}

Effectively delivering integrated patient care and business transactions within the healthcare ecosystem requires the exchange of patient-related data between stakeholders within and across organisations, in order to support clinical decision making and organisational functions such an insurer reimbursement. ${ }^{1}$ The process of transferring or sharing this information using information and communications technologies is often referred to as electronic health information exchange (HIE). ${ }^{2,3}$

Much of the current narrative around HIE has been centred around the United States (US) policy context, arising from the Health Information Technology for Economic and Clinical Health (HITECH) Act. This aims to improve the efficiency, consistency, safety and equity of healthcare services through better information sharing between relevant stakeholders, contingent upon the 'meaningful use' of electronic health records (EHRs), and is backed by a programme of financial incentives and penalties to encourage change.,4 These developments have drawn particular attention to HIE in the US, although the same activities are being undertaken internationally. The screenshot in Figure 1 shows the number of global Google searches, since 2004, for the specific term 'HIE' (lower line) and the topic more generally, which is computed from several indicators (upper line).

Much of the web traffic shown in Figure 1 is driven from the US and closely reflects the 'meaningful use' agenda, which has important but somewhat different implications for clinicians, healthcare delivery organisations, insurers and healthcare information technology providers. It also reflects the explicit use of the search term 'HIE', which is close-coupled with the US policy and business environment.

Although the term HIE is widely used, the way in which it is used varies in significant ways across stakeholders and contexts, including policymakers, academic institutions, professional organisations and funding bodies. Examples of diversity include 'occupational' $\mathrm{HIE},{ }^{4} \mathrm{HIE}$ as a feature of the $\mathrm{EHR}^{5}$ and HIE as a corporate network of data warehouses serving a health organisation. ${ }^{6}$ This raises the potential for ambiguous or inconsistent definitions to hinder meaningful dialogue around health informatics policy, research and practice. For this reason, we decided to undertake a detailed scoping review in order to analyse the ways in which the term is being interpreted in the literature and how these vary across different organisations and contexts. The research therefore set out to answer the question "What is HIE?" with reference to the ways in which it has been defined and the concepts underpinning these definitions. The approach was inspired by earlier scoping reviews of eHealth ${ }^{7,8}$ and aimed to clarify the different uses of the term to in order aid the sharing of information about HIE across stakeholders and inform emerging taxonomies in health informatics. ${ }^{9,10}$

\section{METHODS}

\section{Design}

Systematic scoping review of the published and grey literature. ${ }^{11}$

\section{Inclusion criteria}

Definitions appearing in print or digital format were searched that explicitly refer to HIE or equivalent terms such as clinical information exchange and healthcare information exchange, as well as related concepts such as electronic data interchange (EDI). 'Definition' was taken to include explicit statements of the meaning of the term with reference to key concepts (e.g. 'HIE is the movement of patient information across nodes of a health system using health information technologies and standards for data exchange') and conceptual definitions, in the form of functional descriptions of HIE (e.g. 'organisations exchange health information

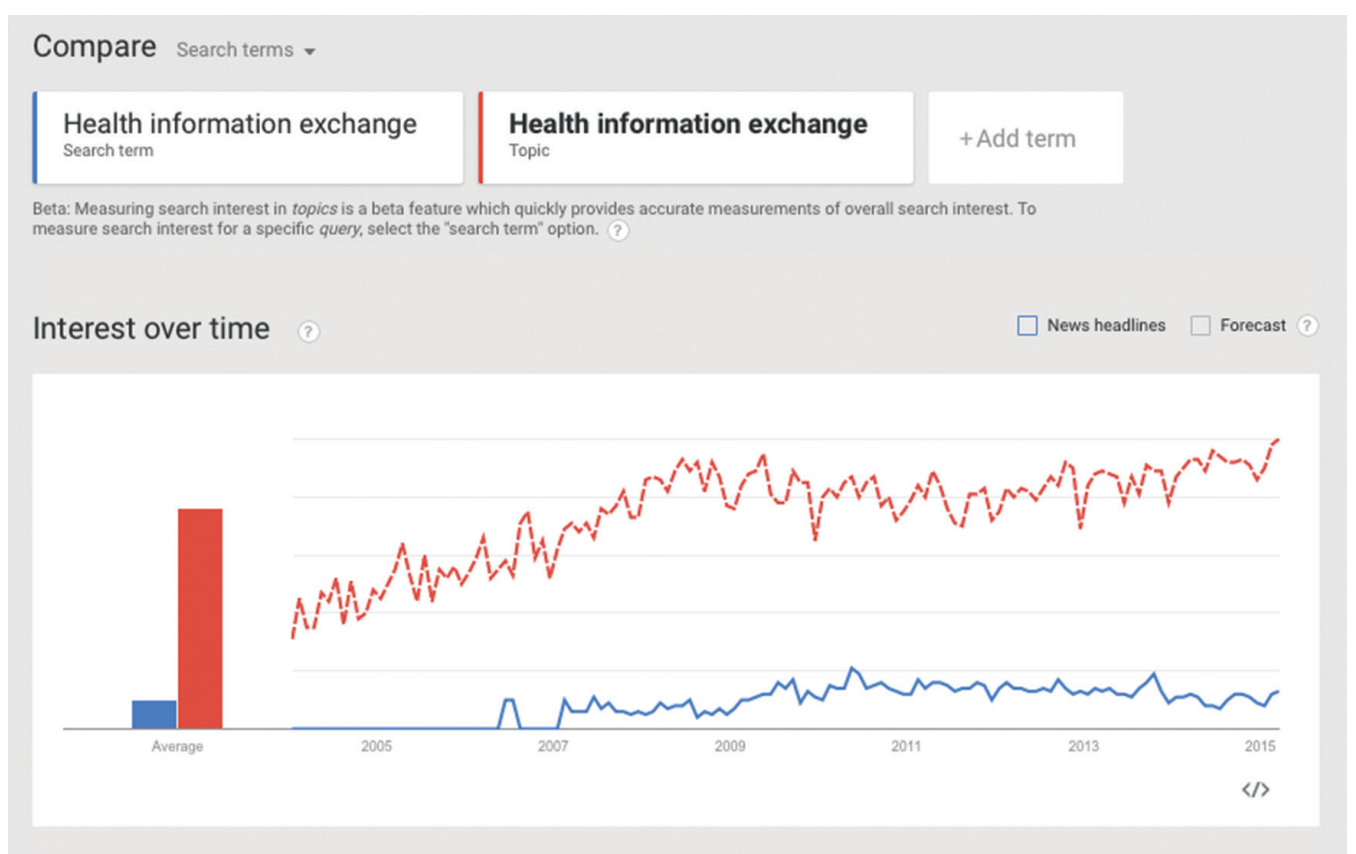

Figure 1 Google searches for HIE as a search string and a topic, since 2005.

Source: Google Trends. Computed on 22 March 2015 
using middleware systems and interoperability standards such as HL7').

\section{Exclusion criteria}

References to HIE not accompanied by either an explicit or an implied definition (as above) were excluded.

\section{Search strategy}

Searches were not limited by language. The searches covered the period from 1900 to February 2014.

Web searches: AA and CP first conducted a Google search using the narrative terms shown in Table 1.

\section{Scientific databases}

The explicit search terms and structured queries shown in Table 2 were used to interrogate Medline; Web of Science; Library Information Science and Technology Abstracts; EMBASE; and CINAHL Plus. There was no medical subject heading $(\mathrm{MeSH})$ term available for HIE, clinical information exchange or healthcare information exchange and hence the reliance on key words.

Query strings were adapted as necessary for each academic database and for the purposes of Google searching. Titles, abstracts and text of the citations identified in the academic database were independently reviewed by two authors (AA and $C P$ ) before articles were retrieved for further analysis. Any disagreements between the investigators were resolved by a third reviewer (AS).

Reference lists of the included articles were also scrutinised for any relevant sources and cited work. Websites included reports, encyclopaedias, dictionaries, blogs, hospitals, vendors, letters, presentations, documents, white papers, articles and references. Duplicate citations and definitions were excluded from the results.
Table 1 Summary of Google searches

\begin{tabular}{|c|c|c|c|}
\hline Search string & Hits & $\begin{array}{l}\text { Total } \\
\text { results } \\
\text { viewed }\end{array}$ & $\begin{array}{c}\text { Unique } \\
\text { definitions } \\
\text { included }\end{array}$ \\
\hline $\begin{array}{l}\text { What is health } \\
\text { information exchange } \\
\text { definition }\end{array}$ & $33,000,000$ & $\begin{array}{c}1000 \\
\text { (100 pages) }\end{array}$ & 61 \\
\hline HIE definition & $32,200,000$ & $\begin{array}{c}1000 \\
\text { (100 pages) }\end{array}$ & 13 \\
\hline What is HIE? & $38,900,000$ & $\begin{array}{c}997 \\
\text { (100 pages) }\end{array}$ & 35 \\
\hline $\begin{array}{l}\text { What is HIE (health } \\
\text { information } \\
\text { exchange) }\end{array}$ & $2,160,000$ & $\begin{array}{c}991 \\
\text { (100 pages) }\end{array}$ & 42 \\
\hline $\begin{array}{l}\text { Clinical information } \\
\text { exchange }\end{array}$ & $20,300,000$ & $\begin{array}{c}993 \\
\text { (100 pages) }\end{array}$ & 10 \\
\hline $\begin{array}{l}\text { Health care information } \\
\text { exchange }\end{array}$ & $111,000,000$ & $\begin{array}{c}1000 \\
\text { (100 pages) }\end{array}$ & 13 \\
\hline
\end{tabular}

\section{Data extraction}

Data extraction tables were prepared to include details of the definition, author, source, year and country of origin. Duplicates appearing in more than one database were excluded prior to further analysis.

\section{Analysis}

As this was a scoping review of definitions, rather than a systematic review of research evidence, analysis did not involve appraisal of study quality. $8,12-14$

All potentially relevant articles identified by the search were analysed by hand in order to determine whether they contained an explicit definition of the term or an equivalent conceptual definition. Definitions were descriptively

Table 2 Summary of academic database searches

\begin{tabular}{|c|c|c|c|c|c|}
\hline Database & Search string & Keyword/topic & Hits & $\begin{array}{l}\text { Results after } \\
\text { duplication } \\
\text { removed }\end{array}$ & $\begin{array}{l}\text { Unique definitions } \\
\text { included }\end{array}$ \\
\hline Medline & $\begin{array}{l}\text { 'Health information exchange' or } \\
\text { 'clinical information exchange' or } \\
\text { 'health care information exchange' }\end{array}$ & Keyword & 400 & 23 & 14 \\
\hline Web of science & $\begin{array}{l}\text { 'Health information exchange' or } \\
\text { 'clinical information exchange' or } \\
\text { 'health care information exchange' }\end{array}$ & Topic & 408 & 50 & 30 \\
\hline $\begin{array}{l}\text { Library information } \\
\text { science and } \\
\text { technology } \\
\text { abstracts }\end{array}$ & $\begin{array}{l}\text { 'Health information exchange' or } \\
\text { 'clinical information exchange' or } \\
\text { 'health care information exchange' }\end{array}$ & Keyword & 81 & 70 & 2 \\
\hline EMBASE & $\begin{array}{l}\text { 'Health information exchange' or } \\
\text { 'clinical information exchange' or } \\
\text { 'health care information exchange' }\end{array}$ & Keyword & 460 & 293 & 69 \\
\hline CINAHL plus & $\begin{array}{l}\text { 'Health information exchange' or } \\
\text { 'clinical information exchange' or } \\
\text { 'health care information exchange' }\end{array}$ & Keyword & 230 & 167 & 9 \\
\hline
\end{tabular}


analysed according to publication date, geographical origin, provenance, and terminology used. AA undertook a high-level pass through the data to create a draft coding framework, which was refined in consultation with CP before beginning the coding process. ${ }^{8,12}$ Definitions were then thematically analysed, with each definition coded according to its various qualitative attributes. The codes were iteratively adapted in order to achieve the best fit with the data, equivalent to qualitative research, where coding proceeds until the point of 'saturation' where no new codes were required to describe the data.

\section{RESULTS}

The Google search yielded 5981 Web pages referring to the term HIE, whilst the searches of scientific databases revealed 603 articles referring to HIE (see Figure 2). These were analysed in order to determine whether they contained an explicit or conceptual definition.
Table 3 shows the number of definitions (see also Appendix A) extracted from Google and scientific databases.

Definitions dated back to the year $1957 .{ }^{15}$ Definitions ranged in length from 1116 to 146 words. ${ }^{17}$ Most of the definitions were from the US. See Table 4 for the number of definitions from various countries and regions. Other terms closely related to HIE found were 'electronic data interchange', 18-20 'clinical information exchange', ${ }^{21-26}$ 'clinical document exchange', 27 'clinical HIE', 28,29 'healthcare information exchange', ${ }^{30-35}$ 'medical data exchange,36 and 'information exchange. 37

\section{KEY THEMES IDENTIFIED}

The objective of facilitating exchange of health information among different systems or stakeholders was represented in the majority of definitions (See Appendices A and B).

The analysis of the included definitions revealed 11 main conceptual themes. In Table 5, these are arranged according to their frequency in the corpus of included definitions, while

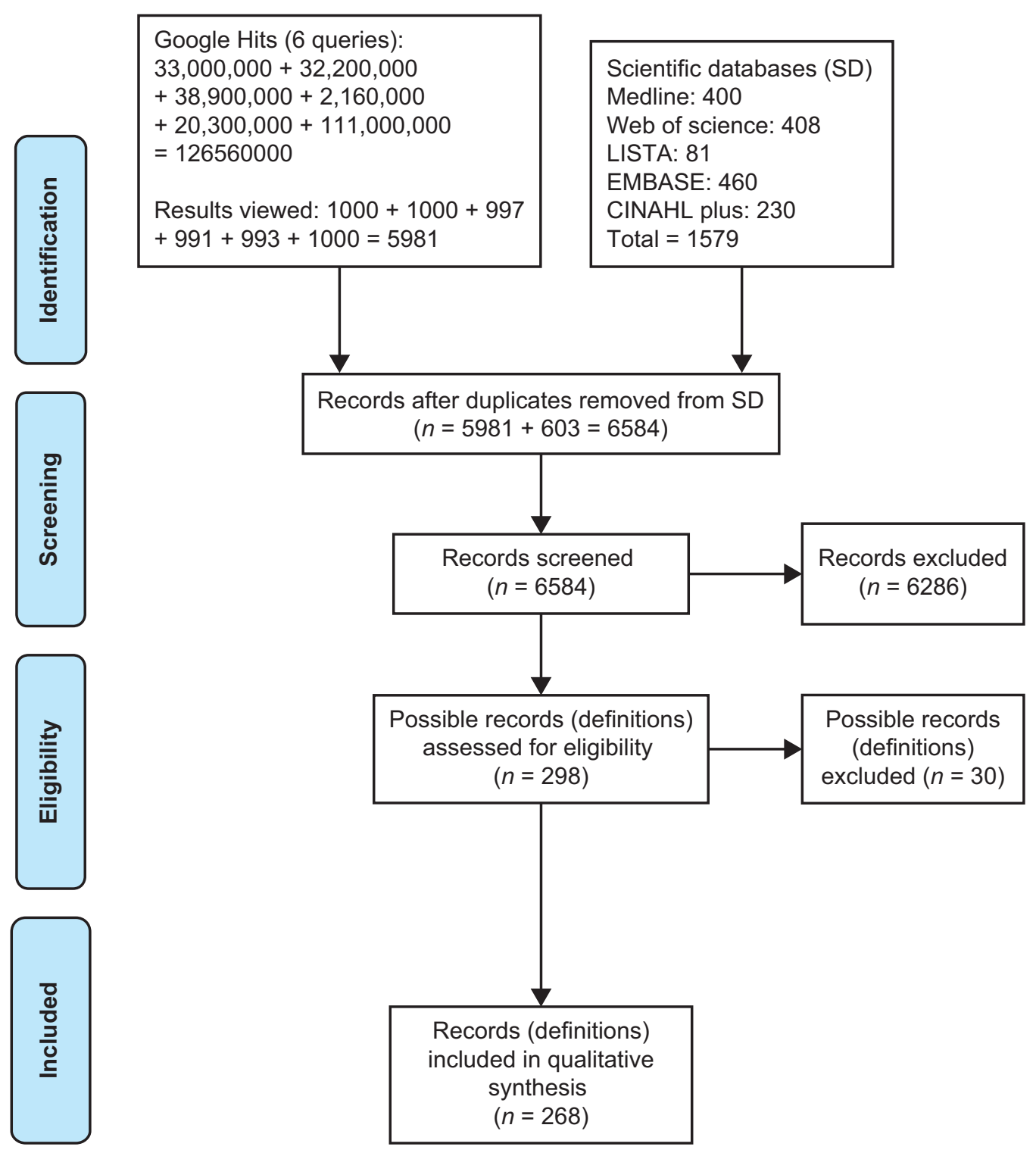

Figure 2 PRISMA diagram of HIE scoping review 
Table 3 The number of definitions retrieved and extracted

\begin{tabular}{lccc}
\hline & Google & $\begin{array}{c}\text { Scientific } \\
\text { databases }\end{array}$ & Total \\
\hline $\begin{array}{l}\text { Eligible definitions } \\
\begin{array}{l}\text { Ineligible } \\
\text { descriptions }\end{array}\end{array}$ & 165 & 103 & 268 \\
\hline Total & 9 & 21 & 30 \\
\hline
\end{tabular}

Table 4 The number of HIE definitions from different countries and regions

\begin{tabular}{ll}
\hline Country & Number of definitions \\
\hline United States & $n=240$ \\
United Kingdom & $n=5^{22,38-41}$ \\
Australia & $n=3^{20,42,43}$ \\
The Netherlands & $n=3^{5,19,44}$ \\
Canada & $n=2^{45,46}$ \\
Denmark & $n=1^{47}$ \\
European Union & $n=1^{48}$ \\
Germany & $n=1^{49}$ \\
Israel & $n=1^{50}$ \\
New Zealand & $n=1^{49}$ \\
Portugal & $n=1^{51}$ \\
Sweden/Finland & $n=1^{52}$ \\
Switzerland & $n=1^{53}$ \\
(Unknown) & $n=7$ \\
\hline
\end{tabular}

Figure 3 represents them in terms of themes and sub-themes (not in the order of frequency). A more detailed breakdown of subthemes and their frequencies may be found in Appendix $B$.

(Many definitions embedded a range of concepts and were thus tagged with more than one code or theme.)

The concepts represented within each higher order theme are described below.

\section{Usage of data and information}

The theme 'data and information' was divided into sub-categories that included 'healthcare information', 'health records', 'claims and administrative' and 'demographic data.'

\section{Health care information}

Definitions referring to the types of information that could be shared, accessed or exchanged are given in Box 1.
Table 5 Themes evolved and the frequency of their occurrence

\begin{tabular}{lc}
\hline Themes & $\begin{array}{c}\text { Frequency of occurrence } \\
\text { in \% }\end{array}$ \\
\hline Usage of 'data and information' & $91 \%$ \\
'Information transfer as a function' & $89 \%$ \\
'Stakeholders' involved in HIE & $75 \%$ \\
'Potential benefits' of HIE & $69 \%$ \\
'Technology' required for HIE & $40 \%$ \\
'Focus'/ 'Context of use' & $34 \%$ \\
'Data standards' & $30 \%$ \\
Connectivity between different & $26 \%$ \\
'locations' & \\
'Public health' outcomes & $12.6 \%$ \\
'Business'solutions/objectives of HIE & $8 \%$ \\
'Sectoral or Regional Scope' & $3 \%$ \\
\hline
\end{tabular}

\section{Box 1 Terms used to represent healthcare information}

'Health information', 2,5,45,49,54-98 'clinical information', 21,3 $3,37,46,61,71,88,97,99-120$ 'clinical data', $23,30,33,87,110,111,121-131$ 'health data', 17,24,43,69,70,76,100,132-140 'patient data', ${ }^{31,33,55,107,141-145 \text { 'healthcare information', } 35,72,84,11}$ 0,115,126,146-148 'clinical patient data', 1,149 'patient clinical data', ${ }^{150}$ 'patient health information', ${ }^{151-158}$ 'patient information', 16,26,29,40,41,47,66,97,116,159-165 'medical information', 21,28, 34,97,107,129,166-171 'health-related information', 16,81,82,121,163, 172-182 'patient-medical information', 183,184 'personal health information', 185 'clinical and demographic data', 186,187 'healthcare-related data', ${ }^{188-190}$ 'health surveillance data', 191 'clinical and other patient data', 145 'surgical procedures', 42 'International Classification of Diseases'42 and 'diagnostic codes and record episodes.' 42

\section{Health records}

Healthcare information was categorised into various types of health records as given in Box 2.

\section{Box 2 Terms used to represent types of health records}

'Paper records', 161 'provider health records' and 'state registries', 79 'digital medical records', 192 'electronic medical record' (EMR), ${ }^{113,165,191,193-198}$ 'EHR' , 5,57,67,81,107,136,162,169,179,199-209 'health information records', ${ }^{130}$ 'care records', 210 'personal health record' (PHR), ${ }^{114,194}$ 'patient health records', 211 'electronic patient records'212and 'patient clinical records.' 51

Further, the contents of healthcare information and health records included are given in Box 3 . 


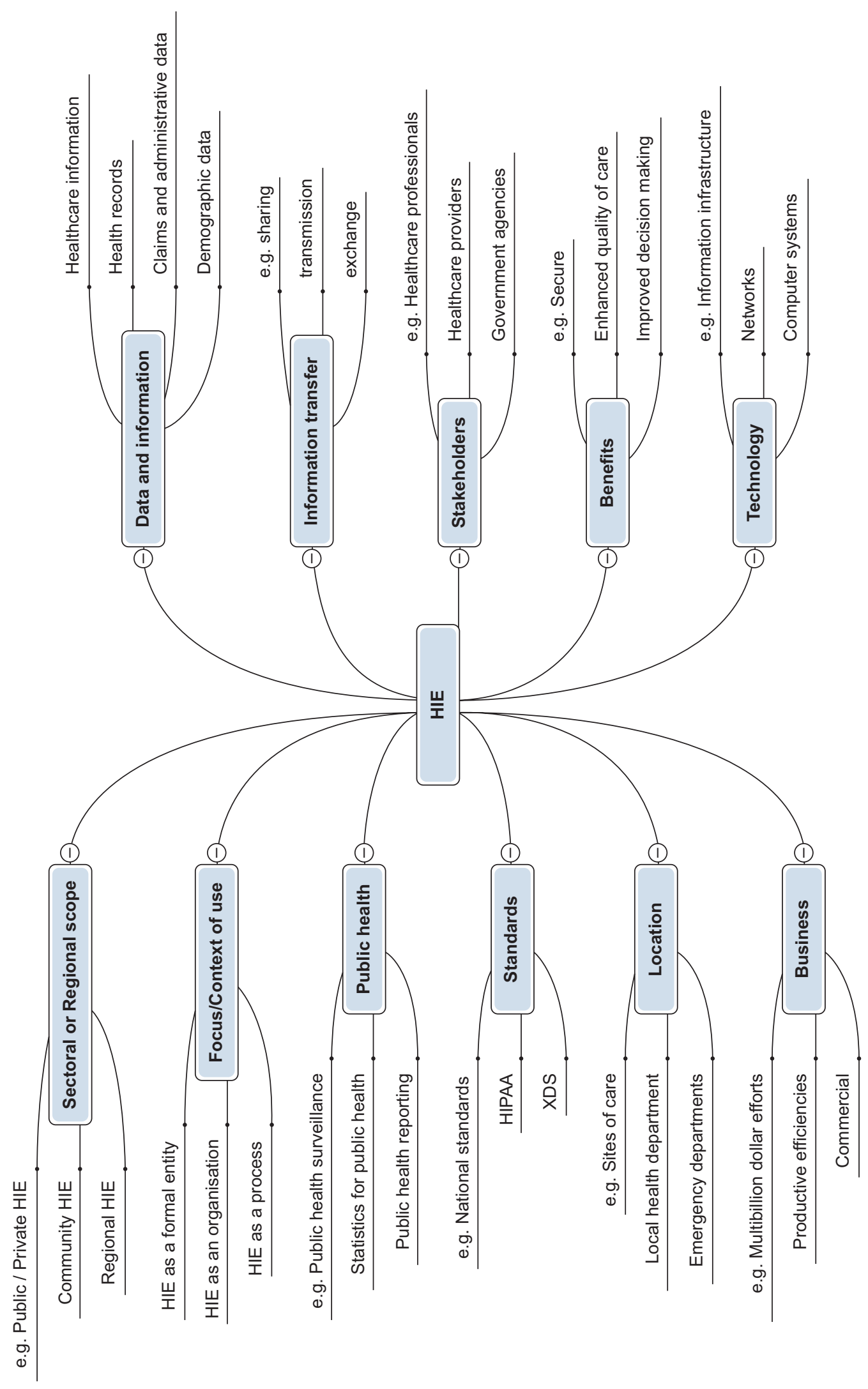

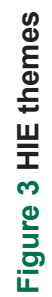




$\begin{aligned} & \text { Box } 3 \text { Terms used to represent 'contents' of healthcare } \\ & \text { information and health records }\end{aligned}$
'Problem lists', 41,105 'illness', 96,185 'injuries', 96
'procedures', ${ }^{42,105,126,158,184}$ 'test results', $88,96,105,185,213,214$
'radiology reports', $88,207,215,216$ 'allergies', $88,96,105,131,185$
'images' and prescriptions, ${ }^{41,184}$ 'medicines', 96,185
'medications' lists, ${ }^{41,88,105,126,131}$
'hospitalization summaries' ${ }^{88}$ and 'discharge
summaries. ${ }^{.94,105,195}$

\section{Claims and administrative data}

Other health information involved claims and administrative data (see Box 4).

\section{Box 4 Terms used to represent claims and administrative data}

Claims related to 'health insurance and payments', ${ }^{20}$ 'claims information'217 and 'health encounter claims', ${ }^{79}$ whereas administrative data were labelled as 'administrative health data', ${ }^{69}$ administrative data, ${ }^{168}$ 'administrative information'117,198 and 'orders.' 165

\section{Demographic data}

Demographic items such as name, date of birth, address and insurance of the patients were exchanged along with the clinical information. ${ }^{27,42,88,186,187}$

\section{Information transfer as a function}

Nearly, all the included definitions referred to health information are being exchanged through electronic processes. Words and expressions used to describe this process or its direction are given in Box 5.

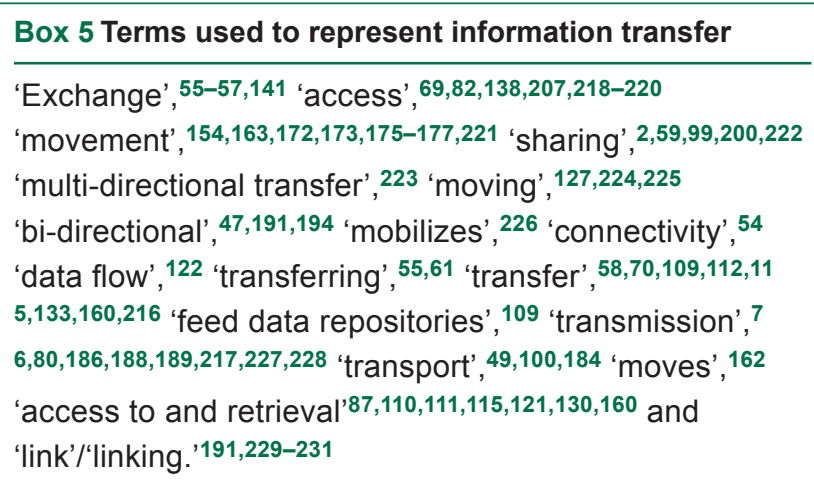

Further, health information was being exchanged and transferred through different mediums and methods such as 'e-transfer', 55 'application-level communication', 49 'email'195,210 and 'secure messaging.' 196

\section{Stakeholders involved in HIE}

The third theme related to the range of stakeholders engaged in HIE, as shown in Box 6:

\section{Box 6 Terms used to represent different types of stakeholders involved in HIE}

'Doctors' $67,69,85,96,142,156,166,179,184,185,232-234$ or

'physicians', 21,77-79,123,144,162,235-237

'clinicians' , 1,56,87,97,99,109,131,135,161,213,218,238-240 'nurses', ${ }^{85,166,184,234,241,242}$ 'patients', 5,38,90,109,133,138,206 'pharmacists', 66,166,184 'health educators', 66 public health officials', 133 'healthcare professionals', ${ }^{29,33,36}$ 'medical professionals', 90 'insurers', ${ }^{142}$

'payers', ${ }^{69,77,175,217,243}$ 'policy makers', 233 'social workers', ${ }^{66}$ 'healthcare providers', 25,58,90,105,126,141,152,154,170,196,197,205, 208,222,230,244 'customers', 44 'hospitals', 19,23,99,101,120,123,222,245 'health plans', $21,67,78,79,171$ 'laboratories', 67,77,85,246 ، radiology centres', 88 'pharmacies', 33,67,85,131,142,162,163,183,235,246,247 'hospitals', 21,32,124,184,195,215,222,237 'organizations', 134,22 3,226,248,249 'institutions', 34,36,44,99,141,250-252 'emergency departments', ${ }^{25,128,142,213}$ 'nursing homes', 101,123 'clinics' (ambulatory/community), ${ }^{32,79,114,120,124,216}$ 'quality assurance groups', 114 'government agencies', ${ }^{18,188-190}$

'state agencies', 79 'public health agencies', ${ }^{84,101,174,175,237,243}$ 'public health facilities', ${ }^{79}$ 'public health entities', ${ }^{114}$ 'health information organizations', ${ }^{188-190}$ 'insurance companies', ${ }^{18,33}$ 'research groups', 114 'public health departments' ${ }^{\prime 23}$ and 'federal reporting entities.'253

Some descriptions of stakeholders were more general, as shown in Box 7:

\section{Box 7 Other terms for stakeholders}

'Stakeholders', 52,68,74,84,106,117,126,127,181,254 "multi-stakeholders", 69,92,195,241 'public-private stakeholders', ${ }^{91}$ 'public health stakeholders', 254 "other or third parties"17,143 and "other healthcare providers." 25,154

\section{Potential benefits of HIE}

Many definitions emphasised the benefits and potential benefits of using HIE, although these were framed in different ways. The dominant benefit was being able to transfer information in a secure way (see Box 8). Other themes related to healthcare quality, timeliness, improved decision making and patient centredness.

Box 8 Terms used to represent potential benefits of HIE

Information security, ${ }^{25,30,43,60,70,89,95,98,100,127,153,157,164,}$ 173,182,191,198,227,234,238,255 'coded', 41,195 improved capacity for informed 'decisions', 17,27,38,47,65,69,83,109, 116,152,229,245,247,253 'effective',21,71,103,115,121,129,160,234,252 'efficient', 33,84,95,121,148,160,233-235 'safer', 37,71,87,110, 111,121,233 'coordinated', 81,234 'equitable', 37,71,110, 111,115,121,129,130 'timelier', 103,121 patient-centred healthcare, ${ }^{37,71,84,110,111,115,121,129,130}$ 'real-time' information transfer, ${ }^{27,99,139,164,198,256}$ enhance 'quality' of healthcare, ${ }^{53,59,106,132,141,213,257}$ reduce costs, ${ }^{2,146,177,216,250,258}$ deliver 'accurate', 97,107,213,247 'seamless', 47,99,140,255 'empowerment' 93 and 'complete'27,97,118,144,150,161,212,222,240,259 health information.

\section{Technology required for HIE}

Almost all of the definitions referred to 'electronic' or 'digital' data exchange. Most referred to the information infrastructure, systems or technology platforms used to host, transfer 
or exchange these data. Other related terms included those in Box 9.

\begin{tabular}{|c|}
\hline Box 9 Terms used to repre \\
\hline 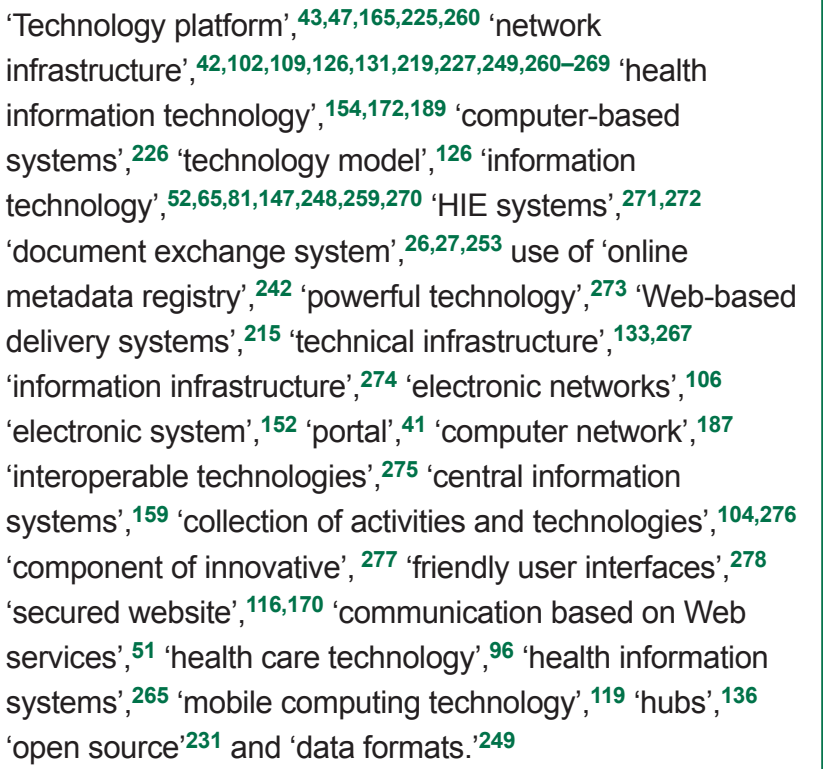 \\
\hline
\end{tabular}

\section{Focus/context of use}

Different definitions also considered HIE with reference to its different contexts of use (see Box 10).

\section{Box 10 Examples of HIE in different contexts of use/focus}

- For instance, 'nursing interventions', 279 'registered nurses' working in home healthcare improving the quality of care through HIE, ${ }^{241}$ contributing to 'emergency departments'24,119,124,142,161,213,239 and focusing on preventive and outcome-based medicines. ${ }^{158}$

- HIE was also used for occupational health, ${ }^{15}$ mental health, ${ }^{280}$ HIV $^{66,230}$ cancer care ${ }^{31}$ and cardiac surgery. ${ }^{119}$

- It is also referred as a 'set of activities' to transfer information from one place to another, ${ }^{201,281}$ as a component of eHealth, ${ }^{43,246}$ as a method of utilising $\mathrm{EHR}^{81,144,169,204,209}$ or electronic patient record ${ }^{212}$ and as an important requirement of Stage 1 'meaningful use' for hospitals. ${ }^{75,105,203,209,282}$

HIE was seen as an entity, organisation as well as a process as described below.

\section{HIE as an entity or organisation}

In many definitions, HIE was labelled as an 'ent ity' $60,70,73,74,77,83,143,227,283$ that was established to exchange health information in a secure manner. It has also been prefixed as 'legal entity' 60 and 'person or governmental entity.' ${ }^{227}$ In some definitions, HIEs were conceived as 'organisations'186,284 or sets of organisational 'services' and infrastructures to support or streamline the exchange of health information. ${ }^{72,149,165,197,206,285,75,263,267,130}$ Other organisational concepts/terms used under this theme are given in Box 11).
Box 11 Examples of HIE used as an entity or organisation

- Here HIE was used as a 'noun', to describe an entity responsible for the exchange of health information. ${ }^{186,217,284}$ The term HIE was synonymously interchanged with local health information organisations, ${ }^{181}$ regional health information organisations (RHIOs) ${ }^{23,45,50,63,72,102,181,207,286}$ and sub-network organisations, ${ }^{181}$ though different names but serving the same purpose. Others perceived that HIEs were run and controlled by $\mathrm{RHIOs},{ }^{63}$ and were funded by healthcare delivery organisations. ${ }^{117}$

- HIEs were occasionally recognised as multi-stakeholder organisations responsible for managing business and legal matters involved in the exchange of information. ${ }^{69}$ Moreover, HIEs were even regarded as the foundational part of accountable care organisations (ACOs) ${ }^{113}$ because they allowed EMRs to exchange information between various types of medical records. The other way around, HIEs were important for organisations that are transitioning into ACOs. ${ }^{94}$

\section{HIE as a process}

Here HIE was considered as the procedure involved in transferring electronic or digital health data in a protected environment among multi-stakeholders. ${ }^{68,81,145,178,180,200,223,224,235,261,283,284}$ Moreover, it was the process of utilising technology and systems to capture, store, share and retrieve the data electronically. ${ }^{35,48}$ In a few definitions, HIE was also reflected as a 'verb', an action, a process responsible for transmitting health information ${ }^{17,186,217,284}$ and collaboration among providers. ${ }^{21,32,94,117,171}$

\section{Data standards}

Incompatibility between systems and software has presented a barrier to HIE, and for this reason, the importance of interoperability standards is emphasised in the majority of definitions. Keywords and phrases related to this are given in Box 12.

\section{Box 12 Terms used to represent the use of data standards}

'Standards', 180,201 'nationally recognized standards', 100,121,13 7,154,173,175-177,228,283 'nationally recognized vocabularies', 195 'policies and standards', 133 'standardise', 149,152,208,254 'standardized content', 49 'standard procedures', 129 'nationwide standards', 283 'national standards', 153,188-190 standards-based infrastructure', 30 'standard-based exchange/solution', 36,238 'cross enterprise document sharing (XDS)'186 to 'Health Insurance Portability and Accountability Act (HIPAA) standards.' ${ }^{89}$

\section{Connectivity between different 'locations'}

Definitions falling within this theme emphasised the exchange of information among facilities or organisations located in different areas (see Box 13). 


\section{Box 13 Terms used to represent different locations}

Such as within a 'region', 2,21,52,69,72,76,87,110,115,145,147, $162,163,171,231,233,257$ or at a 'regional level', $5,40,44,55,75,82$, $83,271,283$ 'community', 2,31,69,82,102,109,124,165,247,287 'non-clinical settings', 66 'metropolitan regions', 124 'sites of care', 150 'delivery settings', ${ }^{54,59}$ 'city', 233 'state/statewide', 21,40,82,131,156,164,168,191,203,205,233 'member sites', 267 'geographic area', 51,68,72,74,138,145 'provincial', 40 'local level/local health departments', 32,75,139,271 'national level', 40,44,75,271 'across countries', 76 'neighbourhood/neighbouring states', 168,283 'multi-state'83 and 'nationwide. '57,256,283

\section{Public health outcomes}

The uses of HIE in population health were stressed in a number of definitions. ${ }^{97,127,165,255,288}$ Relevant concepts covered the uses of HIE for supporting public and community health practices, population health monitoring and research (see Box 14).

\section{Box 14 Terms used to represent public health functions of HIE}

Concepts included connecting 'public health departments', 122,139 supporting 'public health practices', 254 undertaking 'public heath activities', ${ }^{150}$ linking 'public health surveillance data', ${ }^{191}$ 'public health reporting', ${ }^{148,253}$ evaluation and planning of 'public health services', ${ }^{148}$ 'public health event-monitoring capability', 256 'public health situational awareness', 256 analysing the 'health of the population', ${ }^{110}$ 'countrywide population health', ${ }^{115}$ 'statistics for public health', ${ }^{72}$ assist 'public well-being', ${ }^{283}$ protecting 'public interest', 91 'public health organizations', 32 promote 'population health', ${ }^{65,109,141,148,243}$ health data on a 'population level', 139 'track population health', 253 refine 'US health care system', ${ }^{56,74}$ HIE for a '16-county region'171 and 'community betterment'. 87,168

\section{Business solutions/objectives of HIE}

A number of definitions conceived of HIE as either a commercial solution facilitating information exchange or in terms of its business objectives. ${ }^{289}$ It was suggested that HIE will come up with their viable business plans when stimulus funding from the government will end. ${ }^{290}$ See more examples of HIE business solutions/objectives are given in Box 15.

\section{Box 15 Examples of HIE as business solutions/ objectives}

- One vendor represented itself as a national HIE 'company', ${ }^{291}$ while other definitions emphasised issues like scalability and efficiency. ${ }^{258}$

- Siemens offered a four-dimensional HIE 'solution', organised according to 'economics', 'care', 'service' and 'technology.'198
- Transactions between providers or between services or suppliers often involve secure data exchange. ${ }^{17,100}$ This may be conceived in terms of 'data supplier and data receiver' 17 but also 'competitors' 43 and 'customers. 44

- HIE has been spoken about in terms of exchanging health information across traditional business boundaries ${ }^{62}$ and also characterized as 'large multi-billion dollar efforts. 252

- HIE has also been characterised as an infrastructure that includes business models, technology model and the legal model to facilitate the exchange of information among various organisations. ${ }^{91,126}$

- The 'primary business focus' of networks involved in building HIEs was to create value for the provider community by forming 'productive efficiencies.'102

- One of the authors termed HIE as entities built on 'bilateral legal agreements' between proprietary information systems to be able to share different kinds of data. ${ }^{177}$ HIE is seen as an administrative as well as a clinical tool, ${ }^{27,117,168}$ and as a tool for exchanging insurance information. ${ }^{292}$

\section{Sectoral or regional scope}

Many definitions conceived HIE in terms of sectoral, geographic or occupational scope (see Box 16)

\section{Box 16 Terms used to represent sectoral or regional scope of HIE}

'Public' HIE, 165 'private' HIE,, 165 'local' HIE, 133,150 'regional' $\mathrm{HIE},{ }^{133,215}$ 'national' HIE, ${ }^{291}$ 'nationally accessible electronic record', ${ }^{39}$ and 'community' HIE. ${ }^{214}$

\section{DISCUSSION}

The analysis has revealed considerable variability among existing definitions of HIE and insights into the range of concepts that are embedded within these definitions.

Many definitions included not only the exchange of patient information but also other types of information such as demographics, claims and administrative data. Definitions also described health information sharing and connectivity at the level of organisations, states or regions and nations, possibly reflecting the different ways in which care is organised in different national contexts.

While the US government has provided incentives for the adoption of HIE, securing long-term funding is a major challenge for sustainability. Implementing HIE represents an investment and not simply a cost and this is accompanied by a realisation that policy makers must find approaches to assure that all stakeholders who benefit from HIE pay to support it and specifically that efforts should be made to engage private payers. ${ }^{293}$

A wide variety of stakeholders are engaged in $\mathrm{HIE}$, not only including healthcare professionals and patients but also policy makers, researchers, and insurance companies, 
for example. Although infrastructural, economic, technical and cultural barriers have impeded the participation of some stakeholder groups in HIE efforts, it remains the core of the vision for a connected, coordinated and learning health system. ${ }^{294}$

Not surprisingly, the majority of definitions highlighted the importance of secure information exchange and many pointed to the requirement for common data standards necessary for the quality of data content, clinical documentation, data mapping and, most importantly, interoperability between disparate systems. ${ }^{201}$ Common standards will also ensure data protection, confidentiality, interoperability and privacy. $^{29,129,189,228}$

The value of HIE for supporting public health is referred to in many of the definitions, with the ability to meaningfully move clinical data across disparate systems being seen as vital for effective health surveillance and management of care delivered outside health facilities. ${ }^{295}$

\section{Strengths and limitations}

This review aimed to identify, classify and map definitions of HIE and was not intended as an in-depth analysis of the challenges and opportunities presented by HIE, although such issues were inevitably reflected in many of the definitions. The search terms used to identify definitions of HIE were limited to those that we considered most relevant and it is possible that extending them to include other pertinent concepts in health informatics might have revealed further descriptions of HIE and HIE-like concepts. However, we have followed previous authors in prioritising the search for explicit definitions, as a means of generating insights into how the concepts has evolved and how it is being discussed. ${ }^{8,12}$ Using both research databases and a general web search engine gave us greater scope to reach both academic and non-academic literature. Internet searches were restricted to Google and while it is possible that additional definitions may have been found using other search engines, the very large number of hits produced by our queries suggests that it was an effective means of identifying the majority of relevant content in the online grey literature.

The included definitions reflect the dominance of the US literature pertaining to HIE and, to some extent, the language used to describe information sharing concepts in that national context. We acknowledge this limitation, interpreting it as a consequence of the major investment in HIE in the US over recent years, with its inevitable knock-on effects on the volume of literature emerging from that part of the world. As the term gains wider international currency and other countries prioritise investments in HIE, this national balance may change. Nevertheless, a post-hoc analysis excluding the US definitions revealed similar themes as found across our entire corpus of 268 definitions, which encompasses research preHIPPA, suggesting that that the concept and function of HIE is similar in different parts of the world and has consistencies over time (Table 6).
Table 6 Weightage of themes excluding US definitions

\begin{tabular}{lc}
\hline Themes & $\begin{array}{c}\text { Frequency of occurrence } \\
\text { in } \%\end{array}$ \\
\hline Usage of 'data and information' & $90 \%$ \\
'Information transfer as a function' & $90 \%$ \\
'Stakeholders' involved in HIE & $71.4 \%$ \\
'Technology' required for HIE & $57 \%$ \\
'Potential benefits' of HIE & $47.6 \%$ \\
'Data standards' & $28.5 \%$ \\
Connectivity between different & $23.8 \%$ \\
'locations' & \\
'Focus'/ 'context of use' & $23.8 \%$ \\
'Business' objectives of HIE & $9.5 \%$ \\
'Public health' outcomes & $4.7 \%$ \\
\hline
\end{tabular}

\section{Implications for research, practice and policy}

The definitions of HIE appearing in the literature vary on a number of philosophical and conceptual attributes, and many are context specific. The ways in which different authors choose to describe concepts is inevitably influenced by their backgrounds and vested interests and it is therefore not surprising to observe the differences in emphasis. However, we were struck by the clear alignment of the HIE concept with specific technologies or business opportunities in some definitions, where HIE is used describe a service, an organisation or a technology, rather than a process or concept. Such a language was often used by businesses promoting their products or in the context of state information systems, where 'the exchange' is pictured as an operational entity. This creates important obstacles to communication about HIE, particularly for policymakers involved in making decisions about strategy and procurements.

We have not found a single definition that encompasses all of the key attributes of HIE identified by our thematic analysis. The one suggested by Finn ${ }^{69}$ is one of the most comprehensive and differentiates 'HIE' as a concept related to the processes of data transfer and sharing, and 'HIEs' as entities responsible for overseeing effective HIE, while also recognising the benefits, scope and stakeholders involved. ${ }^{69}$ The broad concept of HIE also describes a set of enabling technologies that support these processes, including data standards and public health objectives. The use of HIE as a noun describing organisational entities concerned with mediation and governance of information transfers is largely, although not exclusively, a US phenomenon and also qualitatively different from process-oriented definitions. It has therefore has been placed in separate sentence. Based on our review, we proposed the following integrated definition of HIE shown in Box 17, which is a heavily adapted version of the one proposed by Finn. ${ }^{69}$ 


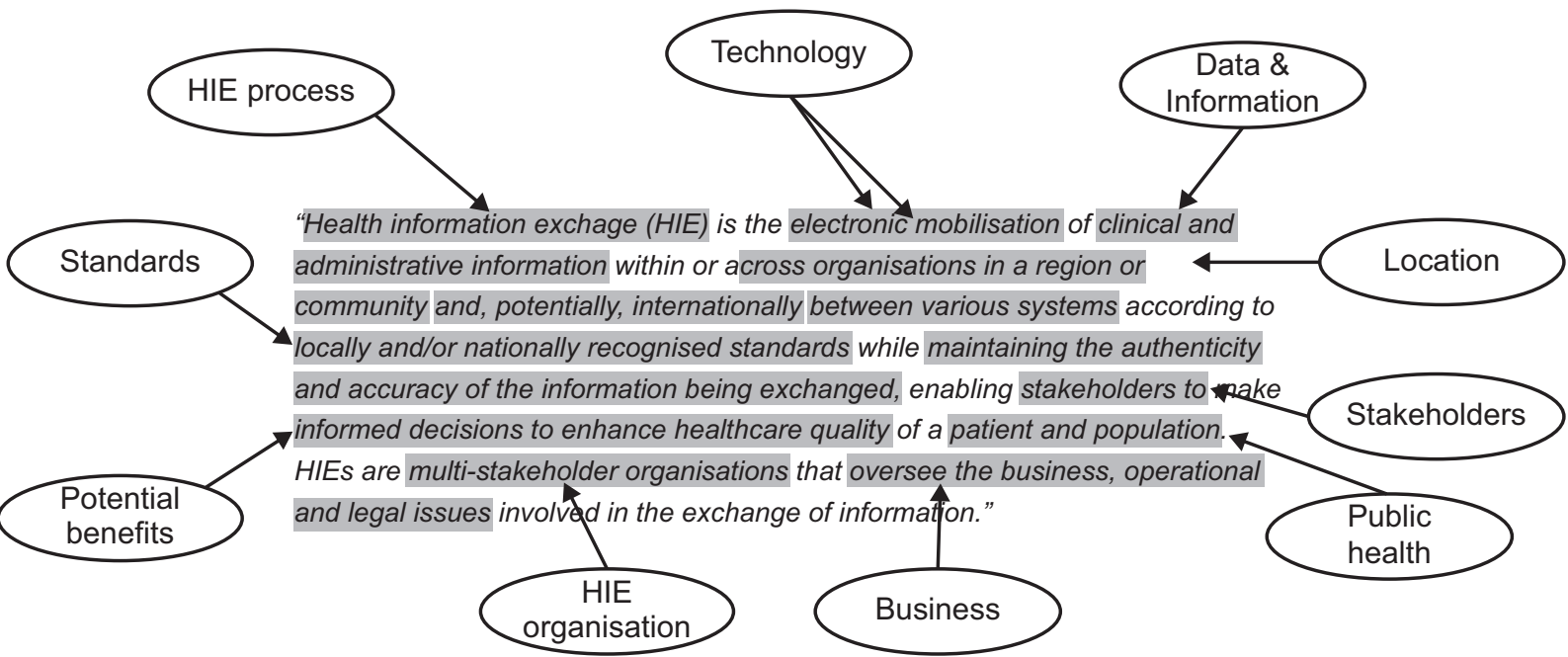

Figure 4 Proposed HIE definition composed of themes identified

Box 17 Adapted and proposed HIE definition
'HIE is the electronic mobilisation of clinical and administra-
tive information within or across organisations in a region
or community and, potentially, internationally between
various systems according to locally and/or nationally
recognised standards while maintaining the authenticity
and accuracy of the information being exchanged, ena-
bling stakeholders to make informed decisions to enhance
healthcare quality of a patient and population. HIEs are
multi-stakeholder organisations that oversee the business,
operational and legal issues involved in the exchange of
information.'

While this definition may not be ideally suited to all contexts (such as HIE for specific diseases and/or community HIE), we believe it provides a good overall fit with the themes identified in the heterogeneous corpus of definitions revealed by our review and therefore propose it for possible wider adoption (see Figure 4).

\section{REFERENCES}

1. Wright A, Soran C, Jenter CA, Volk LA, Bates DW andSimon SR. Physician attitudes toward health information exchange: results of a statewide survey. Journal of the American Medical Informatics Association 2010;17(1):66-70.

2. Overhage JM, Evans L and Marchibroda J. Communities' Readiness for Health Information Exchange: The National Landscape in 2004. Journal of the American Medical Informatics Association 2005;12(2):107-12.

3. Smith PC, Araya-Guerra R, Bublitz C, Parnes B, Dickinson $\mathrm{LM}$, Van Vorst $\mathrm{R}$ et al. Missing clinical information during primary care visits. Journal of the American Medical Association 2005;293(5):565-71.

4. Byers DH. Occupational-Health Information Exchange. Public Health Reports. 1957;72(12):1077-8.

5. Delfan A. Regional Health Information Exchange (RHIE). Thesis. 2013

6. Barton MB, Gabriel GS, Frommer MS, Holt PE and Thompson JF. Surgical procedures for melanoma in public and private New South Wales Hospitals, 2001-2002. ANZ Journal of Surgery 2006;76(5):318-24.

\section{CONCLUSIONS}

This review has shed light on the different facets of HIE. In the spirit of promoting further scholarly discussion and debate, we have proposed a definition that encompasses the key underpinning constructs. We recommend that future authors consider the findings of this review before developing new definitions of $\mathrm{HIE}$, in order to avoid adding further heterogeneity. We also hope the review has value for policymakers involved in planning, procurement and evaluation of HIE.

\section{Funding}

We would like to thank Higher Education Commission Pakistan and The University of Edinburgh for providing funds for this review.

\section{Acknowledgement}

We wish to record our appreciation to Professor Brian McKinstry and the anonymous reviewers for their comments on an earlier draft of this manuscript.

7. Pagliari C, Sloan D, Gregor P, Sullivan F, Detmer D, Kahan JP et al. What is eHealth (4): a scoping exercise to map the field. Journal of Medical Internet Research 2005;7(1).

8. Oh H, Rizo C, Enkin M and Jadad A. What is eHealth (3): a systematic review of published definitions. Journal of Medical Internet Research 2005;7(1).

9. Boonstra A and Broekhuis M. Barriers to the acceptance of electronic medical records by physicians from systematic review to taxonomy and interventions. BMC Health Services Research 2010;10(1):231.

10. Castillo VH, Martínez-García Al and Pulido J. A knowledgebased taxonomy of critical factors for adopting electronic health record systems by physicians: a systematic literature review. BMC Medical Informatics and Decision Making 2010;10(1):60.

11. Arksey $\mathrm{H}$ and O'Malley L. Scoping studies: towards a methodological framework. International Journal of Social Research Methodology 2005;8(1):19-32. 
12. Van De Belt TH, Engelen LJ, Berben $S A$ and Schoonhoven $L$. Definition of Health 2.0 and Medicine 2.0: a systematic review. Journal of Medical Internet Research 2010;12(2).

13. Sood S, Mbarika V, Jugoo S, Dookhy R, Doarn CR, Prakash N et al. What is telemedicine? A collection of 104 peer-reviewed perspectives and theoretical underpinnings. Telemedicine and e-Health 2007;13(5):573-90.

14. Frank JR, Mungroo R, Ahmad $Y$, Wang M, De Rossi $S$ and Horsley T. Toward a definition of competency-based education in medicine: a systematic review of published definitions. Medical Teacher 2010;32(8):631-7.

15. Byers $\mathrm{DH}$. Occupational health information exchange. Public Health Reports. 1957;72(12):1077.

16. AlaskaeHealth. What is health information exchange?: Alaska eHealth Network. Available from: http://ak-ehealth.org/forpatients/what-is-health-information-exchange/. Accessed 30 December 2013.

17. Committee $\mathrm{H}$. HIE Implications in Meaningful Use Stage 1 Requirements. Healthcare Information and Management Systems Society (HIMSS). 2010. Available from: https://www. onehealthport.com/sites/default/files/hie/MU_HIE_Matrix.pdf

18. Braithwaite WR, Ed. The federal role in setting standards for the exchange of health information,1998 Proceedings Pacific, Medical Technology Symposium, IEEE, 1998.

19. Hasman A, Ament A, Arnou P and Van Kesteren A. Interinstitutional information exchange in healthcare. International Journal of Bio-Medical Computing 1992;31(1):5-16.

20. Regan B. Computerised information exchange in health care. Medical Journal of Australia 1991;154(2):140.

21. HealtheLink. About Healthelink. Available from: http://wnyhealthelink.com/WhatWeDo/HEALTHeLINKNews/

Wyoming CountyCommunity Health System Joins HEALTHeLINK. Accessed 20 January 2014.

22. Payne TH, Detmer DE, Wyatt JC and Buchan IE. National-scale clinical information exchange in the United Kingdom: lessons for the United States. Journal of the American Medical Informatics Association 2011;18(1):91-8.

23. Grinspan ZM, Berg L, Onyile A, Kaushal R and Shapiro J. Medical information fragmentation for people with epilepsy in new york city differs by type of visit. Epilepsy Currents 2013;13:315.

24. Onyile A, Shapiro JS and Kuperman G. Patient crossover rates vary by disease in a health information exchange. Annals of Emergency Medicine. 2011;58(1):S294-S5.

25. Onyile A, Vaidya SR, Kuperman G and Shapiro JS. Geographical distribution of patients visiting a health information exchange in New York City. Journal of the American Medical Informatics Association 2013;20:e125-e30.

26. Hazamy PA, Haley V, Tserenpuntsag B and Tsivitis M. Using remote access to audit hospital data submitted to NHSN for reporting of hospital acquired infections in New York State. American Journal of Infection Control 2013;(1):S76.

27. Iatric. Clinical Document Exchange. Available from: http://www. iatric.com/ClinicalDocumentExchange. Accessed 15 January 2014.

28. Utah. Utah Statewide Clinical Health Information Exchange 2011. 2011

29. StateUtah. Utah Clinical Health Information Exchange. 2011.

30. Infor_Clover. Infor Cloverleaf Hosted Healthcare Information Exchange. Available from: http://www.infor.com/product_summary/healthcare/cloverleaf-hies/ Accessed 12 December 2013.

31. Merrill M. Georgia Cancer Coalition uses HIE to share evidence-based medicine 2009. Available from: http:// www. healthcareitnews.com/news/georgia-cancer-coalition-uses-hie-share-evidence-based-medicine. Accessed 5 January 2014.
32. SanDiegoHIE. San Diego Regional Healthcare Information Exchange. Available from: http://www.sandiegobeacon.org/ san-diego-beacon-community.

33. SafeNet. What You Need to Know About Securing Healthcare Information Exchanges. 2010. Available from: https://www. safenet-inc.com/resources/security-guide/data-protection/ What_You_Need_to_Know_About_Securing_Healthcare_ Information_Exchanges___Security_Guide/.

34. Birkle M, Schneider B, Beck T, Deuster T, Fischer M, Flatow F et al. Implementation of an open source provider organization registry service. Studies in Health Technology and Informatics. 2011;169:265-9.

35. Cannoy SD. Consumer Empowerment in Healthcare Information Exchange: An Investigation Using the Grounded Theory Approach. Greensboro:The University of North Carolina, 2008.

36. Cisco. Cisco Medical Data Exchange Solution. Available from: http://www.cisco.com/web/strategy/healthcare/medical_data_ exchange_solution.html. Accessed 15 February 2014.

37. MHiE. Physician Information Exchang. Available from: http:// www.memorialhermann.org/healthcare-professionals/physician-information-exchange. Accessed 15 January 2014.

38. Bugge C, Entwistle VA and Watt IS. The significance for decision-making of information that is not exchanged by patients and health professionals during consultations. Social Science and Medicine 2006;63(8):2065-78.

39. Greenhalgh T, Morris L, Wyatt JC, Thomas G and Gunning K. Introducing a nationally shared electronic patient record: Case study comparison of Scotland, England, Wales and Northern Ireland. International journal of Medical Informatics. 2013.

40. Boyle T. Privacy Lessons Learned from an Operational Health Information Exchange. FairWarnings, Inc., 2011. Available from: http://www.fairwarning.com/privacy-lessons-learned-from-anoperational-health-information-exchange-nhs-scotland/.

41. Excelicare. One of the world's leading Health Information Exchange (HIE) applications. Available from: http://www.axsys. co.uk/excelicare-clinical-portal/. Accessed 15 January 2014.

42. Barton MB, Gabriel GS, Frommer MS, Holt PE and Thompson JF. Surgical procedures for melanoma in public and private New South Wales hospitals, 2001-2002. ANZ Journal of Surgery 2006;76(5):318-24.

43. CSC. Health Information Exchange: CSC. Available from: http://www.isofthealth.com/en-au/Solutions/ANZ\%20HIE.aspx. Accessed 26 December 2013.

44. Forcare. Software solutions for Health Information Exchanges. Available from: http://www.forcare.com/solutions/health-information-exchanges-hie/. Accessed 26 December 2013.

45. Rebryna R. EHR Arithmetic: Extracting Positives from Negatives. The Santa Barbara County Care Data Exchange Project. Healthcare Quarterly. 2009;7(4):82-5.

46. Sicotte $C$ and Pare $G$. Success in health information exchange projects: Solving the implementation puzzle. Social Science and Medicine 2010;70(8):1159-65.

47. InterSystem. Denmark Selects InterSystems HealthShare for Countrywide Health Information Exchange 2012. Available from: http://www.ehealthnews.eu/intersystems/2936-denmarkselects-intersystems-healthshare-for-countrywide-health-information-exchange. Accessed 20 February 2014.

48. Codagnone C. Benchmarking deployment of eHealth Among General Practitioners II. 2013.

49. HISO. Health Information Exchange Architecture Building Blocks, Overview and Glossary. Health Information Standards Organzation 2012 HISO 10040.0.

50. Frankel M, Chinitz D, Salzberg CA andReichman K. Sustainable health information exchanges: the role of institutional factors. Israel Journal of Health Policy Research 2013;2(1):21.

51. Alert. Alert $₫$ Health Information Exchange. Available from: http://www.alert-online.com/hie. Accessed 8 January 2014. 
52. Mäenpää $T$, Asikainen $P$, Gissler $M$, Siponen $K$, Maass $M$, Saranto $\mathrm{K}$ et al. Outcomes Assessment of the Regional Health Information Exchange. Methods of Information in Medicine 2011;50(4):308-18.

53. Geissbuhler A. Lessons learned implementing a regional health information exchange in Geneva as a pilot for the Swiss national eHealth strategy. International Journal of Medical Informatics. 2013.

54. Adler-Milstein J, DesRoches CM and Jha AK. Health information exchange among US hospitals. American Journal of Managed Care 2011;17(11):761.

55. Adler-Milstein J, Ronchi E, Cohen GR, Winn LAP and Jha AK. Benchmarking health IT among OECD countries: better data for better policy. Journal of the American Medical Informatics Association. 2014;21(1):111-6.

56. America loMCoQoHCi. Crossing the Quality Chasm: A New Health System for the 21st Century.Washington, DC:National Academies Press, 2001.

57. Carter J. Electronic Health Records: a Guide for Clinicians and Administrators.Sydney: ACP Press, 2008.

58. DC Behavioral Health Association. Mental Health Information and Primary Care Integration Act of 2011. Washington, DC:DC Behavioral Health Association, 2011.

59. Dullabh P, Hovey L. Large scale health information exchange: implementation experiences from five States. Studies in Health Technology and Informatics 2012;192:613-7.

60. Frisse ME. Health Information Exchange in Memphis: Impact on the Physician-Patient Relationship. Journal of Law, Medicine and Ethics 2010;38(1):50-7.

61. Initiative e. 2011 Report on Health Information Exchange: The Changing Landscape. 2011.

62. Hersh W. A stimulus to define informatics and health information technology. BMC Medical Informatics and Decision Making 2009;9(1):24.

63. Hripcsak G, Kaushal R, Johnson KB, Ash JS, Bates DW, Block $R$ et al. The United Hospital Fund meeting on evaluating health information exchange. Journal of Biomedical Informatics 2007;40(6):S3-S10.

64. Kern LM and Kaushal R. Health information technology and health information exchange in New York State: new initiatives in implementation and evaluation. Journal of Biomedical Informatics 2007;40(6):S17-S20.

65. Lobach DF, Kawamoto K, Anstrom KJ, Kooy KR, Eisenstein EL, Silvey GM et al., Ed. Proactive population health management in the context of a regional health information exchange using standards-based decision support. AMIA Annual Symposium Proceedings, 2007.

66. Myers JJ, Koester KA, Chakravarty D, Pearson C, Maiorana A, Shade SB et al. Perceptions regarding the ease of use and usefulness of health information exchange systems among medical providers, case managers and non-clinical staff members working in HIV care and community settings. International Journal of Medical Informatics. 2012;81(10):e21-9.

67. 360 eP. Patient FAQ 2011. Available from: http://www.ohii. ca.gov/calohi/.

68. Foundation R. Health Information Exchange - An Introduction. Available from: http://www.rchnfoundation.org/?p=1757.

69. Finn N. Health information exchange: a stepping stone toward continuity of care and participatory medicine. Journal of Participatory Medicine 2011;3:e47.

70. Assembly IG. (20 ILCS 3860/) Illinois Health Information Exchange and Technology Act. Available from: http://www.ilga. gov/legislation/ilcs/ilcs3.asp?ActID=3267\&ChapterID=5.

71. Afzal S. Maryland's Health Information Exchange, SOA in Healthcare Conference, the Role of Health Information Exchange in Driving Toward Interoperability. Afzal S, Ed.: Chesapeake Regional Information System for our Patients, 2011.
72. PCmag. Definition of:HIE. Available from: http://www.pcmag. com/encyclopedia/term/61628/hie. Accessed 26 December 2013.

73. Office USGA. Electronic Personal Health Information Exchange Health Care Entities' Reported Disclosure Practices and Effects on Quality of Care 2010. Available from: http://www.gao.gov/ new.items/d10361.pdf. Accessed 8 January 2014.

74. AMA. Health Information Technology: American Medical Association. Available from: https://www.ama-assn.org/ama/ pub/advocacy/topics/health-information-technology.page. Accessed 20 December 2013.

75. Aspelin M. What is a Health Information Exchange (HIE)? An Introduction to HIEs. Available from: http://markaspelin.com/ health-information-exchange-hie-primer-hies/. Accessed 5 January 2014.

76. Thomas J. OpenHIE Architecture 2013. Available from: https:// wiki.ohie.org/display/documents/OpenHIE+Architecture. Accessed 16 December 2013.

77. Kongstvedt PR. Essentials of managed health care. Massachusetts:Jones \& Bartlett Publishers,2012.

78. Infor_IBM. IBM Enterprise Master Person Index (EMPI). Available from: http://www.infor.com/product_summary/healthcare/empi/. Accessed 4 January 2014.

79. HIECC/SEPAP. Meaningful Health Information Exchange. 2009.

80. NDHIN. Introduction/Definitions: North Dakota Health Information Network. Available from: http://www.ndhin.org/resource/policies/ introductiondefinitions. Accessed 5 January 2014.

81. L. Block P. Introduction to Health Informatics. Available from: http://www.philblock.info/hitkb/h/health_informatics.html. Accessed 24 December 2014.

82. MSV. Health Information Exchange (HIE): Medical Society of Virginia. Available from: http://www.msv.org/ MainMenuCategories/MemberCenter/Knowledgebase/HIT/ HIE.aspx. Accessed 20 December 2013.

83. Index NHRSAR. 332-I:1 Medical Records; Definitions 1989. Available from: http://nhrsa.org/law/332-i-1-medical-recordsdefinitions/. Accessed 5 January 2014.

84. iNexx. Available from: http://www.inexx.com/health-informationexchange-primer.html. Accessed 10 January 2014

85. PAeHealth. WhatistheDifferencebetweenHIXandHIE?.Available from: http://www.google.co.uk/url?sa=t\&rct=j\&q=\&esrc=s\&frm $=1 \&$ source $=$ web $\& c d=18 \&$ cad $=$ rja $\&$ ved $=0$ CHMQFjAHOAo\&url $=$ http $\% 3 \mathrm{~A} \% 2 \mathrm{~F} \% 2 \mathrm{Fwww}$.portal.state.pa.us $\% 2 \mathrm{Fportal} \% 2 \mathrm{Fserver}$.

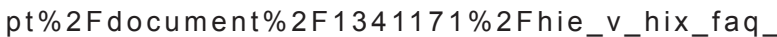
pdf\&ei=ZtaxUre-FOmo0AXDtoGAAQ\&usg=AFQjCNG5hqNTL DGbazT7OziJ1Re7n-D0hA\&sig2=INswc4hAspMfhZNjfjGlgA. Accessed 10 December 2015.

86. HT. Health Information Exchange (HIE). Available from: http://www.cctheart.com/HTPN_HIE_Education.pdf. Accessed 26 December 2013.

87. OnePartnerHIE. What is an HIE? : One Partner HIE. Available from: http://www.onepartnerhie.com/About/WhatisanHIE.aspx. Accessed 10 January 2014.

88. InspiraHN. Health Information Exchange (HIE). Available from: http://www.inspirahealthnetwork.org/?id=5253\&sid=1. Accessed 15 December 2013.

89. LMO. What is HIE? Liquid Medical Office, Inc. Available from: http://www.liquidemr.com/HIE/WhatisHIE.html. Accessed 15 December 2013.

90. Rhodelsland. Health Information Exchange (HIE) Project. Available from: http://www.health.ri.gov/projects/healthinformationexchange/. Accessed 16 December 2013.

91. NV-HIE. Nevada Health Information Exchange. Available from: http://nv-hie.org/. Accessed 24 December 2013.

92. SEMHIE. Southeast Michigan Health Information Exchange. Available from: http://www.semhie.org/. Accessed 23 December 2013. 
93. THHSC. Texas Health And Human Service Commission. Available from: http://www.hhsc.state.tx.us/hhsc_projects/oehc/ StatewideExchange.shtml. Accessed 30 December 2013.

94. Harris. Health Information Exchange Connect community. Provide access. Create collaboration.: Harris Healthcare Solutions. Available from: http://healthcare.harris.com/solutions/hie-healthinformation-exchange.aspx. Accessed 20 December 2013.

95. FloridaHIN. Florida Health Information Exchange Overview. Available from: http://www.fhin.net/content/floridaHie/. Accessed 17 December 2013.

96. Princeton_Health. Frequently Asked Questions About Health Information Exchanges Princeton Healthacre Systems. Available from: http://www.princetonhcs.org/phcs-home/privacy-policy/frequently-asked-questions-about-health-information-exchanges-hie.aspx. Accessed 9 January 2014.

97. OrionHIE. Health Information Exchange. Available from: http:// www.orionhealth.com/health-information-exchange. Accessed 26 December 2014.

98. Matthews T, Leroy L, Rowland T. Indiana and Ohio Health Information Exchanges Connect for Nation's First Live, Multi-Region Clinical Information Exchange 2009. Available from: https://mpcms.blob.core.windows.net/bd985247-f489435f-a7b4-49df92ec868e/docs/4879a229-9f98-4018-ae15ca2bb448c34a/ihie-hb-hl-connection-press-release-final.pdf. Accessed 10 January 2014.

99. Altman R, Shapiro JS, Moore T and Kuperman GJ. Notifications of hospital events to outpatient clinicians using health information exchange: a post-implementation survey. Informatics in Primary Care 2012;20(4):249-55.

100. Bouhaddou O, Bennett J, Teal J, Pugh M, Sands M, Fontaine F et al., Ed. Toward a Virtual Lifetime Electronic Record: The Department of Veterans Affairs Experience with the Nationwide Health Information Network. AMIA Annual Symposium Proceedings, 2012.

101. Daurio NR, Fielding M and Cholewka PA. Implementation of an enterprise-wide Electronic Health Record: a nurse-physician partnership. Studies in Health Technology and Informatics 2009;146:263.

102. De Brantes F, Emery D, Overhage J, Glaser J and Marchibroda J. The potential of HIEs as infomediaries. Journal of Healthcare Information Management 2007;21(1):69.

103. Hincapie AL, Warholak TL, Murcko AC, Slack M and Malone DC. Physicians' opinions of a health information exchange. Journal of the American Medical Informatics Association 2011;18(1):60-5.

104. J. M. Health information exchange. AHA 2011;85(2):22-7.

105. Jones SS, Friedberg MW and Schneider EC, Ed. Health information exchange, Health Information Technology use, and hospital readmission rates. AMIA Annual Symposium Proceedings, 2011.

106. Moore J. Definition of HIE: What's Yours? Chilmark Research, 2011. Available from: http://www.chilmarkresearch. com/2011/01/08/definition-of-hie-whats-yours/. Accessed 26 December 2013.

107. PDN. Health Information Exchange Definition-Defined By Experts. Available from: http://www.medical-record-coding.com/2011/12/health-information-exchange-definition/. Accessed 15 December 2013.

108. Vaidya SR, Shapiro JS, Papa AV, Kuperman G, Ali N, Check $T$ et al. Perceptions of health information exchange in home healthcare. Computers Informatics Nursing 2012;30(9):503-9.

109. Voigt $C$ and Torzewski S. Direct results. An HIE tests simple information exchange using the direct project. Journal of the American Health Information Management Association 2011;82(5):38-41.

110. Wikipedia. Health information exchange. Available from: http://en.wikipedia.org/wiki/Health_information_ exchange_\%28HIE\%29. Accessed 19 December 2013.
111. CDC. Health Information Exchange: A Key Concept for Biosurveillance. Available from: https://spaces.internet2.edu/ download/attachments/12876/NCPHI-RHIE-CDC-HIE-ViewFCC-version1a.pdf?api=v2.

112. SCA. Healthcare Identity Management: The Foundation for a Secure and Trusted National Health Information Network: Smart Card Allaince,2009. Available from: http://www.smartcardalliance.org/pages/publications-healthcare-identity-management. Accessed 16 December 2013.

113. OPTUM. ACO Glossary of Terms. Available from: http://www. optuminsight.com/accountable-care-organizations/resources/ glossary/. Accessed 8 January 2014.

114. mhhealthcare. Available from: http://blog.mhhealthcare. $\mathrm{com} / \mathrm{tag} / \mathrm{himss}$-health-information-exchange/\%20-\%20 sthash.88sUavsh.dpuf. Accessed 26 December 2013.

115. PDN. The vision, mission(s) and goal(s) of health information exchange that was, is and will remain in effect... forever. Professional Dynamic Network. Available from: http://www. pdnseek.com/Blog/Resource-Pages/Health-InformationExchange-History.aspx. Accessed 26 December 2013.

116. HIE_Bridge. Health Information Exchange. Available from: http://www.hiebridge.org/. Accessed 9 January 2014.

117. Gartner. Health Information Exchange (HIE). Available from: http://www.gartner.com/it-glossary/hie. Accessed 8 January 2014.

118. Committee on Enhancing the Internet for Health Applications: Technical Requirements and Implementation Strategies, CSaTB, National Research Council. Networking Health: Prescriptions for the Internet.Washington, DC: The National Academies Press, 2000.

119. Gassert CA. Defining information requirements using holistic models: introduction to a case study. Holistic Nursing Practice 1996;11(1):64-74.

120. Liu S. Enabling Electronic Healthcare Information Exchange. IEEE IT Professional 2007;9(6):17-23.

121. HRSA. What is health information exchange? U.S. Department of Health and Human Services, Health Information Technology and Quality Improvement. Available from: http://www.hrsa.gov/ healthit/toolbox/RuralHealthITtoolbox/Collaboration/whatishie. $\mathrm{html}$. Accessed 15 January 2014.

122. Adler-Milstein J, Bates DW and Jha AK. A survey of health information exchange organizations in the United States: implications for meaningful use. Annals of Internal Medicine 2011;154(10):666-71.

123. Adler-Milstein $\mathrm{J}$ and Jha AK. Sharing clinical data electronically: a critical challenge for fixing the health care system. Journal of the American Medical Association. 2012;307(16):1695-6.

124. Gadd CS, Ho Y-X, Cala CM, Blakemore D, Chen Q, Frisse ME et al. User perspectives on the usability of a regional health information exchange. Journal of the American Medical Informatics Association 2011;18(5):711-6.

125. Kern LM, Dhopeshwarkar R, Barrón $Y$, Wilcox $A$, Pincus $H$ and Kaushal R. Measuring the effects of health information technology on quality of care: a novel set of proposed metrics for electronic quality reporting. Joint Commission Journal on Quality and Patient Safety 2009;35(7):359-69.

126. Lee L, Whitcomb K, Galbreth $M$ and Patterson D. A strong state role in the HIE. Lessons from the South Carolina Health Information Exchange. Journal of AHIMA 2010;81(6): 46-50, quiz 1.

127. Reeder B, Revere D, Hills RA, Baseman JG and Lober WB. Public Health Practice within a Health Information Exchange: Information Needs and Barriers to Disease Surveillance. Online journal of public health informatics 2012;4(3).

128. Shapiro JS. Evaluating public health uses of health information exchange. Journal of Biomedical Informatics 2007;40(6):S46-S9. 
129. Michigan. Michigan Health Information Technology Commission 2007-2008 Report to the Michigan Legislature. 2008.

130. MTBC. HIE, the future of electronic medical records?. Available from: http://www.mtbc.com/learningcenter/index.php/hie-thefuture-of-electronic-medical-records/. Accessed 25 December 2013.

131. Centricity. GE Healthcare to Grow its Health Information Exchange Services by Joining Global eHealth Leader 2009. Available from: http://www.ehealthserver.com/ge/245-gehealthcare-to-grow-its-health-information-exchange-servicesby-joining-global-ehealth-leader. Accessed 15 Februrary 2014.

132. Kuperman GJ. Health-information exchange: why are we doing it, and what are we doing? Journal of the American Medical Informatics Association 2011;18(5):678-82.

133. Sittig DF and Joe JC. Toward a statewide health information technology center (abbreviated version). Southern Medical Journal 2010;103(11):1111-4.

134. Unertl KM, Johnson KB and Lorenzi NM. Health information exchange technology on the front lines of healthcare: workflow factors and patterns of use. Journal of the American Medical Informatics Association 2012;19(3):392-400.

135. Vreeman DJ. Keeping up with changing source system terms in a local health information infrastructure: running to stand still. Studies in Health Technology and Informatics 2007;129 (Pt 1):775.

136. Rowley R. Rethinking health information exchange 2013. Available from: http://robertrowleymd.com/2013/08/21/rethinking-health-information-exchange/. Accessed 10 December 2013.

137. HHS. National Biosurveillance Strategy for Human Health V2.0. US Department of Health and Human Services, 2010.

138. C. Kibbe D. Responses to 'Meaningful Use': a pivotal definition for new-wave medical records systems 2009. Available from: http://e-patients.net/archives/2009/05/meaningful-use-apivotal-definition-for-new-wave-medical-records-systems.html. Accessed 3 February 2014.

139. KC. Health Information Exchange (HIE): Kane County Health Department. Available from: http://kanehealth.com/hie.htm. Accessed 30 December 2013.

140. Oracle. Oracle Health Information Exchange: Secure, Seamless Data Sharing. 2012. Available from: http://www.oracle.com/us/ industries/healthcare/health-information-exchange-br-195127. pdf.

141. Ancker JS, Edwards AM, Miller MC and Kaushal R. Consumer Perceptions of Electronic Health Information Exchange. American Journal of Preventive Medicine 2012;43(1):76-80.

142. Genes N, Shapiro J, Vaidya S and Kuperman G. Adoption of Health Information Exchange by Emergency Physicians at Three Urban Academic Medical Centers. Applied Clinical Informatics 2011;2(3):263.

143. KLAS. Health Information Exchange (HIE). 2014. Available from: http://www.klasresearch.com/segment/198. Accessed 20 December 2013.

144. IHS. Health Information Exchange (HIE) Indian Health Service. Maryland: Indian Health Service. Available from: http://www. ins.gov/hie/index.cfm?module=dsp_hie_faq. Accessed 20 December 2013.

145. Schulte M. Healthcare Delivery in the U.S.A.: An Introduction2012.Boca Raton, Florida: CRC Press, 2012.

146. Hall C. What is a Health Information Exchange?2009. Georgia: Centers for Disease Control and Prevention. Available from: http://www.cap.org/apps/docs/committees/informatics/Health_ Information_Exchange.pdf.

147. STHL. STHL HIE|Frequently Asked Questions (FAQs): Southern tier healthlink New York. Available from: http://www. sthlny.com/FAQs.asp. Accessed 10 January 2014.
148. Merrill JA, Deegan M, Wilson RV, Kaushal R and Fredericks K. A system dynamics evaluation model: implementation of health information exchange for public health reporting. Journal of the American Medical Informatics Association 2013;20(e1):e131-e8.

149. Mearian L. Verizon creates medical information exchange cloud 2010. Available from: http://www.computerworld.com/s/ article/9179182/Verizon_creates_medical_information_ exchange_cloud. Accessed 20 February 2014.

150. Grossman JM, Kushner KL and November EA. Creating sustainable local health information exchanges: can barriers to stakeholder participation be overcome? Research Brief, 2008;(2):1-12.

151. HIE Anwers. Health Information Exchange Models. Available from: http://www.hieanswers.net/hie-fundamentals/hie-models/. Accessed 20 December 2013.

152. NC_HIE. North Carolina Health Information Exchnage. Available from: http://nchie.org/nc-hie/about/. Accessed 25 December 2013

153. FloridaHIE. The Florida HIE. Available from: https://www.floridahie.net/. Accessed 20 December 2013.

154. HIE_Texas. HIE Texas. Available from: http://www.hietexas. org/local-hies/32-providers/providers-accordion/135-what-ishealth-information-exchange-hie. Accessed 10 January 2014.

155. 1PartnerHIE. What is OnePartner HIE? OnePartner HIE. Available from: http://www.onepartnerhie.com/. Accessed 16 December 2013.

156. RIQI. Current Care: Rhode Island Quality Institute. Available from: http://www.riqi.org/matriarch/MultiPiecePage.asp_Q_ PagelD_E_94_A_PageName_E_HIELearn. Accessed 13 December 2013

157. MaineGov. Maine's Health Information Exchange (HIE). Available from: http://www.maine.gov/dhhs/oms/HIT/hie.htm. Accessed 24 December 2013.

158. Xerox. Healthcare Information Exchange (HIE). Available from: http://services.xerox.com/informed-health/healthcare-itsolutions/health-information-exchange/enus.html. Accessed 20 Janurary 2014.

159. Clemens NA. Privacy, consent, and the electronic mental health record: The person vs. the system. Journal of Psychiatric Practice 2012;18(1):46-50.

160. Gaebel H. What is an HIE? 2010. Available from: https://himsshie.pbworks.com/w/page/4775490/HIEDefinition. Accessed 12 Janurary 2014.

161. Corhio. HIPAA and Health Information Exchange. Colorado Regional Health Information Organization.

162. HC. HIE FAQ: Greater Houston Health Connect. Available from: http://ghhconnect.org/index.html\#/what-is-an-hie----faq/. Accessed 15 December 2013.

163. World_Privacy_Forum. Health Information Exchanges in California. Available from: http://www.worldprivacyforum. org/2013/08/hie/. Accessed 14 December 2013.

164. ArkansasOHIT. State Health Alliance for Records Exchange (SHARE). Available from: http://ohit.arkansas.gov/share/Pages/ default.aspx. Accessed 27 December 2013.

165. Accenx. Accenx: A rapid, cost-effective on-ramp to Health Information Exchange: Healthcare Informatics 2009. Available from: http://www.healthcare-informatics.com/article/accenxrapid-cost-effective-ramp-health-information-exchange. Accessed 10 January 2014.

166. HealthIT. Health Information Exchange (HIE). Available from: http://www.healthit.gov/HIE. Accessed 10 January 2014.

167. Joshi JK. Clinical Value-Add for Health Information Exchange (HIE). The Internet Journal of Medical Informatics 2010;6(1).

168. AxolotlCorp. NeHII Completes Demonstration of Statewide Health Information Exchange 2009. Available from: http://finance. paidcontent.org/paidcontent/news/read?GUID=9343105. Accessed 10 January 2014. 
169. Recogniti. Health Information Exchange (HIE). Available from: http://recogniti.com/solutions/hie/. Accessed 10 December 2013.

170. Routzon J. Blog. Available from: http://cihie.org/\#/ blog/4561389177/HIE.-Yes-please/4754309. Accessed 10 January 2014.

171. HIXNY. HIXNY achieves strategic interoperability with community practices. Available from: http://www.intersystems.com/ library/library-item/healthcare-information-xchange-of-newyork-hixny-leverages-intersystems-healthshare-to-increaseprovider-adoption-community-engagement/. Accessed 20 January 2014.

172. NAHIT. Defining Key Health Information Technology Terms. Washigton DC: Office of the National Coordinator for Health Information Technology. National Alliance for Health Information Technology, 2008.

173. HIMSS. Health Information Exchanges Part 2: Putting the HIE into Practice. Available from: https://www.himss.org/sites/himssorg/files/HIMSSorg/Content/files/HIMSS_HIE_Presentation_ PuttingHIEPractice.pdf. Accessed 26 December 2013.

174. OCI. Building a Health Information Exchange for the State of Missouri. Missouri:Object Computing, Inc., 2009.

175. Bostick R, Crayton G, Fishman E, Elaine P and Vern S. Sustaining Health Information Exchange: A State Toolkit. NGA Centre for Best Practices, 2011.

176. AHIMA. HIE. Available from: http://www.ahima.org/resources/ hie.aspx. Accessed 15 December 2013.

177. Pennsylvania. Pennsylvania eHealth Collaborative Strategic Plan for Health Information Exchange 2012. 2012. Available from: https://www.paehealth.org/images/pdf/PA_eHealth_ Strat_Plan_2012_Final.pdf. Accessed 26 December 2013.

178. 1stProvidersChoice. Healthcare Information Exchange Certified EMR Software, Leading Fully Certified EMR Software already ready for the Heathcare Information Exchange Available from: http://www.1stproviderschoice.com/emr-healthcare-information-exchange.php. Accessed 26 December 2013.

179. HINAz. What is Health Information Exchange? : Health Information Network of Arizona Available from: http://www. hinaz.org/what. Accessed 4 January 2014.

180. IGI Global. What is Health Information Exchange (HIE). Available from: www.igi-global.com/dictionary/ health-information-exchange-hie/12859.

181. Amatayakul M. HIEs: survival of the fittest: an estimated 130 health information exchange (HIE) organizations exist today-and many of these fledgling HIEs are at risk. (INSIDE IT) 2008. Available from: http://www.highbeam.com/doc/1G1-192352076. html. Accessed 22 December 2014.

182. Louisiana. What is the Louisiana Health Information Exchange? Available from: http://www.lhcqf.org/lahie-about. Accessed 10 Februrary 2014.

183. Suenaga G. About the State HIE 2012. Available from: https:// www.hawaiihie.org/posts/section.state_hie_about. Accessed 20 December 2013.

184. CareAccord. Health Information Exchange in Oregon. Available from: https://www.careaccord.org/hie-in-oregon/overview. shtml. Accessed 15 December 2013.

185. Virtua. Health Information Exchange (HIE). Available from: https://www.virtua.org/patient-tools/health-informationexchange. Accessed 22 December 2013.

186. SuccessEHS. What is HIE? Fast Facts about Health Information Exchange. Available from: http://successehs.com/item/what-ishie.htm. Accessed 5 January 2014.

187. VITL. HIE Overview. Available from: http://www.vitl.net/healthinformation-exchange/what-is-hie. Accessed 10 January 2014.

188. 4Med. What is HIE and HIPAA Compliance?. Available from: http://www.4medapproved.com/hit108.php. Accessed 16 December 2013.
189. HealthCareIT. Health Information Exchange (HIE). Available from: http://www.healthcareitnews.com/directory/health-information-exchange-hie. Accessed 22 December 2013.

190. Vanguard. Get Connected - Provide Timely, Efficient and Effective Patient-Centered Care. Convergent's OmniMD. Available from: https://www.vanguardsys.com/medical/ehr/ health-information-exchange-hie/. Accessed 20 December 2013.

191. Herwehe J, Wilbright W, Abrams A, Bergson S, Foxhood J, Kaiser $\mathrm{M}$ et al. Implementation of an innovative, integrated electronic medical record (EMR) and public health information exchange for HIVIAIDS. Journal of the American Medical Informatics Association 2012;19(3):448-52.

192. HealthLeaders. Kentucky selects ACS to develop health information exchange 2009. Available from: http://www. healthleadersmedia.com/content/TEC-239380/Kentuckyselects-ACS-to-develop-health-information-exchange.html. Accessed 5 January 2014.

193. Luo JS. Electronic health information exchange: Key trends to watch. Primary Psychiatry. 2006;13(5):19.

194. Mcllwain J, Lassetter K. HIE: decision support. Building sustainable HIEs. In the aftermath of Hurricane Katrina, the need for a true health information exchange in Mississippi cannot be denied. Health Management Technology 2009;30(2):8.

195. Loonsk JW. Not All Health Information Exchange Is Created Equal 2010. Available from: http://www.collaborativegov. org/not-all-health-information-exchange-is-created-equal/. Accessed 20 December 2013.

196. MobileMD. MobileMD Health Information Exchange. Available from: http://usa.healthcare.siemens.com/infrastructure-it/ healthcare-it/care-coordination-connectivity/mobile-md-hie-hs/ mobilemd-hie-hs. Accessed 15 December 2013.

197. Palmetto. Health Information ExchangeBetter care. Everywhere. Palmetto Health. Available from: http://www.palmettohealth. org/body-NoRightMenu.cfm?id=4236. Accessed 23 December 2013.

198. MobileMD_Siemens. MobileMD HIE. Available from: http:// usa.healthcare.siemens.com/infrastructure-it/healthcare-it/ care-coordination-connectivity/mobile-md-hie-hs. Accessed 15 December 2013.

199. Hitech. HITECH Act 2009. Washington, DC: Hitech. 2009.

200. Chaudhary O. Developing the Foundation for Syndromic Surveillance and Health Information Exchange for Yolo County, California. Online Journal of Public Health Informatics 2012;4(2).

201. Morrissey J. HIE: Which Way Will You Go? Hospital and Health Networks. 2013. Available from: www.hhnmag.com/ articles/5965-hie-which-way-will-you-go.

202. Shapiro JS, Genes N, Kuperman G, Chason K and Richardson LD. Health information exchange, biosurveillance efforts, and emergency department crowding during the spring 2009 H1N1 outbreak in New York City. Annals of Emergency Medicine 2010;55(3):274-9.

203. ILHIE. Illinois HIE Strategic and Operational Plan 2010. Illinois:ILHIE, 2010.

204. KeyHIE. Better Outcomes Start With Better Information. Available from: http://www.keyhie.org/providers/benefits_overview.html. Accessed 29 December 2013.

205. HealthInsight. Public Perception and Utah's Clinical Health Information Exchange 2011-2012. Available from: https:// healthinsight.org/Internal/docs/chie_consumer_focus_groups_ report.pdf. Accessed 26 December 2013.

206. UWHealth. Electronic Health Information Exchange. Available from: http://www.uwhealth.org/files/uwhealth/docs/pdf4/EHIE. pdf. Accessed 26 December 2013.

207. Stoten S. Health Policy Issue with the Electronic Health Record. Online Journal of Nursing Informatics. 2009;13(2):2. 
208. NCHICA. Healthcare Information Exchange (HIE): Considerations for Engaging North Carolina Consumers. North California:NCHICA, 2009.

209. NAHP. Electronic Health Records National Association for Health Professionals. 2011. https://www.nahpusa.com/userfiles/file/ CEU/final\%200611.pdf. Accessed on 26 December 2013.

210. Reeder G. Meaningful Use Roadmap for HIE 2011-2012. Available from: http://www.ehrdoctors.com/the-meaningful-useroadmap-for-hie/. Accessed 5 January 2014.

211. Kijsanayotin B, Speedie SM and Connelly DP. Linking patients' records across organizations while maintaining anonymity. AMIA Annual Symposium Proceedings 2007;11:1008.

212. Bresnick J. Does HIE cause more confusion than it prevents? 2013. Available from: http://ehrintelligence.com/2013/05/21/ does-hie-cause-more-confusion-than-it-prevents/. Accessed 22 December 2013.

213. Halamka JD. Health information exchange for emergency department care is on the right trajectory. Annals of Emergency Medicine 2013;62(1):25-7.

214. Ross SE, Schilling LM, Fernald DH, Davidson AJ and West DR. Health information exchange in small-to-medium sized family medicine practices: Motivators, barriers, and potential facilitators of adoption. International Journal of Medical Informatics 2010;79(2):123-9.

215. Abhyankar S, Lloyd-Puryear MA, Goodwin R, Copeland S, Eichwald J, Therrell BL et al., Ed. Standardizing newborn screening results for health information exchange. AMIA Annual Symposium Proceedings, 2010.

216. Bredfeldt C. PS2-10: Economic Impact of Electronic Health Information Exchange. Clinical Medicine and Research 2013;11(3):152.

217. Smith LB. HIE 101: Definition 2012. Available from: https://www. thehitcommunity.org/2012/08/hie-101-definition/. Accessed 22 December 2013.

218. Ozkaynak M and Brennan PF. Revisiting sociotechnical systems in a case of unreported use of health information exchange system in three hospital emergency departments. Journal of Evaluation in Clinical Practice 2013;19(2):370-3.

219. Munoz RT, Fox MD and Gomez MR. Presumed Consent Models and Health Information Exchanges: Hard Nudges and Ambiguous Benefits. The American Journal of Bioethics 2013;13(6):14-5.

220. PrivacyRights. California Medical Privacy Fact Sheet C2: How Is Your Medical Information Used and Disclosed -With and Without Consent? 2012. Available from: https://www.privacyrights.org/fs/ fsC2/CA-medical-uses-disclosures. Accessed 26 December 2013.

221. Lori H. What is HIE? a Federal and State Perspective. California: Object Health LLC. Available from: https://www.acgov.org/ board/district3/documents/2012-10-22WhatlsHIE.pdf.

222. Furukawa MF, Patel V, Charles D, Swain M and Mostashari F. Hospital Electronic Health Information Exchange Grew Substantially In 2008-12. Health Affairs. 2013;32(8):1346-54.

223. Shade SB, Chakravarty D, Koester KA, Steward WT and Myers JJ. Health information exchange interventions can enhance quality and continuity of HIV care. International Journal of Medical Informatics 2012;81(10):e1-e9.

224. Vest JR. Health Information Exchange: National and International Approaches. Advances in Health Care Management 2012;12:3-24.

225. InteliChart. Health Information Exchange (HIE): Inteli Chart. Available from: http://www.intelichart.com/informationexchange-overview. Accessed 10 January 2014.

226. Warholak TL, Murcko A, McKee M and Urbine T. Results of the Arizona Medicaid Health Information Technology Pharmacy Focus Groups. Research in Social and Administrative Pharmacy 2011;7(4):438-43.

227. HIE O. Summary of Law on Health Information Exchanges. 2014 Contract No. 15 January. Available from: http://codes. ohio.gov/orc/3798. Accessed 26 December 2016.
228. Minnesota. Health Information Exchange (HIE) Minnesota Department of Health. Available from: http://www.health.state. mn.us/e-health/hie.html. Accessed 10 December 2013.

229. Kijsanayotin B, Speedie SM and Connelly DP, Ed. Linking patients' records across organizations while maintaining anonymity. AMIA Annual Symposium Proceedings, 2007.

230. GeorgiaGov. HIV Health Information Exchange (HIE). Available from: http://dph.georgia.gov/hiv-health-information-exchangehie. Accessed 16 December 2013.

231. eHealth_Open_Source. Hartford Hospital:Open Source Health Information Exchange (HIE): eHealth Open Source. Available from: http://www.ehealthopensource.com/case-studies/hartford-hospitalopen-source-health-information-exchange-hie/. Accessed 8 January 2014.

232. Murphy K. Health Information Exchange 2012. Available from: http://ehrintelligence.com/glossary/health-informationexchange/. Accessed 5 January 2014.

233. Walker J, Pan E, Johnston D, Adler-Milstein J, Bates DW and Middleton $B$. The value of health care information exchange and interoperability. Health Affairs-Millwood 2005;24:W5.

234. Williams C, Mostashari F, Mertz K, Hogin E and Atwal P. From the office of the national coordinator: the strategy for advancing the exchange of health information. Health Affairs 2012;31(3):527-36.

235. Emdeon. Health Information Exchange. Available from: http:// www.emdeon.com/hie/. Accessed 26 December 2013.

236. eHealth4AZ.Arizona. Frequently Asked Questions: Health Information Newtwork of Arizona. Available from: http://www. hinaz.org/patient-faq\#hie. Accessed 26 December 2013.

237. Kralewski JE, Zink T and Boyle R. Factors influencing electronic clinical information exchange in small medical group practices. Journal of Rural Health 2012;28(1):28-33.

238. Cerner. Clinical Exchange Platform. Available from: https:// store.cerner.com/items/28. Accessed 10 January 2014.

239. Shapiro JS, Kannry J, Kushniruk AW, Kuperman G. Emergency physicians' perceptions of health information exchange. Journal of the American Medical Informatics Association 2007;14(6):700-5.

240. Vest JR. Health Information Exchange and Healthcare Utilization. Journal of Medical Systems 2009;33(3):223-31.

241. Vaidya SR, Shapiro JS, Papa AV, Kuperman G, Ali N, Check $T$ et al. Perceptions of health information exchange in home healthcare. Computers Informatics Nursing 2012;30(9):503-9.

242. Whittenburg L. Nursing point of care documentation for the evaluation of human quality. Studies in Health Technology and Informatics 2008;146:713-4.

243. PRISM. Health Information Exchange: PRISM Health Consulting. Available from: http://www.prismcommtest $1 . c o m / s e r v i c e s / h e a l t h-$ information-exchange/. Accessed 15 December 2013.

244. GulfCoastHIE. What is HIE? Available from: http://www.gulfcoasthie.com/. Accessed 15 December 2013.

245. AHA. Health Information Exchange Projects. What Hospitals and Health Systems Need to Know. Available from: www.aha.org/ content/00-10/AHARHIOfinal.pdf. Accessed 26 December 2013.

246. Marchibroda JM. Health Literacy, eHealth, and Communication: Putting the Consumer First: Workshop Summary. Institute of Medicine (US) Roundtable on Health Literacy.Washington, DC: National Academies Press, 2009.

247. HIE_Neveda. Health HIE Neveda. Available from: http://www. healthienevada.org/. Accessed 17 December 2013.

248. Sicotte C, Paré G. Success in health information exchange projects: Solving the implementation puzzle. Social Science and Medicine 2010;70(8):1159-65.

249. Steward WT, Koester KA, Collins SP, Maiorana A and Myers JJ. The essential role of reconfiguration capabilities in the implementation of HIV-related health information exchanges. International Journal of Medical Informatics. 2012; 81(10):e10-20. 
250. C. Livingood W, Coughlin S and Remo R. Public Health \& Electronic Health Information Exchange: A Guide to Local Agency Leadership. Florida:Duval County Health Department, Institute for Public Health Informatics and Research.

251. Haggstrom DA, Doebbeling BN. Quality Measurement and System Change of Cancer Care Delivery. Medical Care 2011;49:S21-S7.

252. Johnson KB and Gadd C. Playing smallball: approaches to evaluating pilot health information exchange systems. Journal of Biomedical Informatics 2007;40(6):S21-S6.

253. CitiusTech. Health Information Exchange | HIE Reporting Overview. Available from: http://www.citiustech.com/markets/ hie.aspx. Accessed 15 December 2013.

254. Grannis SJ, Stevens KC and Merriwether R. Leveraging health information exchange to support public health situational awareness: the Indiana experience. Online Journal of Public Health Informatics 2010;2(2).

255. HealthUnity. Solutions/Statewide HIE. Available from: http:// healthunity.com/Solutions.mvc.aspx/StatewideHIE. Accessed 18 December 2013.

256. Revere D and Stevens KC. Accelerating Public Health Situational Awareness through Health Information Exchanges: An Annotated Bibliography. Online Journal of Public Health Informatics 2010;2(2).

257. ClinicalConnect. ClinicalConnect: A secure health information exchange. Available from: http://www.clinicalconnecthie.com/. Accessed 15 January 2014.

258. Vest JR, Campion Jr TR and Kaushal R. Challenges, alternatives, and paths to sustainability for health information exchange efforts. Journal of Medical Systems 2013;37(6):1-8.

259. Kaelber DC and Bates DW. Health information exchange and patient safety. Journal of Biomedical Informatics 2007;40(6):S40-S5.

260. Professional Dynamics Networks, Inc. Health Information Exchange Definition-Defined By Experts. Available from: http:// www.medical-record-coding.com/2011/12/health-informationexchange-definition/. Accessed 22 December 2013.

261. Tang PC and Lee TH. Your doctor's office or the Internet? Two paths to personal health records. New England Journal of Medicine 2009;360(13):1276-8.

262. Gordon P, Camhi E, Hesse R, Odlum M, Schnall R, Rodriguez $M$ et al. Processes and outcomes of developing a continuity of care document for use as a personal health record by people living with HIVIAIDS in New York City. International Journal of Medical Informatics 2012;81(10):e63-73.

263. Deas Jr TM, Solomon MR. Health information exchange: foundation for better care. Gastrointestinal Endoscopy 2012;76(1):163-8.

264. Thornewill J, Dowling AF, Cox BA, Esterhay RJ. Information infrastructure for consumer health: a health information exchange stakeholder study. American Journal of Preventive Medicine 2011;40(5):S123-S33.

265. Overhage JM. Health information exchange:'lex parsimoniae.' Health Affairs 2007;26(5):w595-w7.

266. Finnell JT and Overhage JM, Ed. Emergency medical services: the frontier in health information exchange. AMIA Annual Symposium Proceedings, 2010.

267. Shapiro JS, Johnson SA, Angiollilo J, Fleischman W, Onyile $A$ and Kuperman G. Health Information Exchange Improves Identification Of Frequent Emergency Department Users. Health Affairs 2013;32(12):2193-8.

268. Moore J. Definition of HIE: What's Yours? Available from: http://thehealthcareblog.com/blog/2011/01/10/definition-of-hiewhat\%E2\%80\%99s-yours/. Accessed 25 December 2013.

269. Rowley R. Rethinking health information exchange. Available from: http://robertrowleymd.com/2013/08/21/rethinking-healthinformation-exchange/. Accessed 25 December 2013.
270. Vimarlund V, Timpka T and Patel VL, Ed. Information technology and knowledge exchange in health-care organizations. Proceedings of the AMIA Symposium,1999.

271. Lloyd-Puryear MA and Brower A. Long-term follow-up in newborn screening: a systems approach for improving health outcomes. Genetics in Medicine 2010;12:S256-S60.

272. Shapiro JS, Kannry J, Kushniruk AW and Kuperman G. Emergency Physicians' Perceptions of Health Information Exchange. Journal of the American Medical Informatics Association 2007;14(6):700-5.

273. Lee S-i, Park H, Kim J-W, Hwang H, Cho E-Y, Kim Y et al. Physicians' Perceptions and Use of a Health Information Exchange: A Pilot Program in South Korea. Telemedicine and e-Health 2012;18(8):604-12.

274. Ben-Assuli O, Shabtai I, Leshno M. The influence of EHR components on admission decisions. Health and Technology 2012:1-7.

275. Joshi J. Clinical Value-Add for Health Information Exchange (HIE). The Internet Journal of Medical Informatics 2011;6(1).

276. Hub HI. OnePartner and MobileMD Collaborate for Health Information Exchange. Available from: http://www.healthimaginghub.com/news/healthcare-it/2615-onepartner-and-mobilemdcollaborate-for-health-information-exchange.html. Accessed 24 December 2013.

277. iHealthTrust. What is a HIE? 2014. Available from: http://www. ihealthtrust.org/content/whatisahie. Accessed 15 January 2014.

278. Elsevier,2009. health care information exchange.

279. Dimitropoulos L and Rizk S. A state-based approach to privacy and security for interoperable health information exchange. Health Affairs 2009;28(2):428-34.

280. Health Care Incentives Improvement Institute. Health Information Exchange: From Start Up to Sustainability. Foundation for eHealth Initiative.Connecticut: Health Care Incentives Improvement Institute, 2007.

281. Vest JR and Jasperson JS. How are health professionals using health information exchange systems? Measuring usage for evaluation and system improvement. Journal of Medical Systems 2012;36(5):3195-204.

282. marchcarson100. 2011. Available from: http://mandmhitech. blog.com/2011/05/26/healthcare-informationexchange-definition-of-an-hie/.

283. Karl ES. What's in a name? Breaking down health information exchange, one definition at a time. Journal of AHIMA 2012;83(6):62-3.

284. Frisse ME, Johnson KB, Nian H, Davison CL, Gadd CS, Unertl $\mathrm{KM}$ et al. The financial impact of health information exchange on emergency department care. Journal of the American Medical Informatics Association 2012;19(3):328-33.

285. McDonald C. Protecting patients in health information exchange: a defense of the hipaa privacy rule. Health Affairs 2009;28(2):447-9.

286. Carter P, Chrisann L, Debra M,Rita B and Hjort B. Privacy and Security in Health Information Exchange. Journal of AHIMA 2006;77(10).

287. Murphy K. Maine HIE puts focus on population health 2012. Available from: http://ehrintelligence.com/2012/09/04/maine-hieputs-focus-on-population-health/. Accessed 22 December 2013.

288. IHIE. Nationwide Health Information Network (NHIN) Trial Implementations. Indiana:Indiana Health Information Exchange, 2009.

289. Kruse CS, Regier $V$ and Rheinboldt KT. Barriers Over Time to Full Implementation of Health Information Exchange in the United States. JMIR Medical Informatics 2014;2(2):e26.

290. ICA. ICA and AlliedHIE Launch National Health Information Exchange ICA,2012. Available from: http://www.icainformatics. com/2012/05/ica-and-alliedhie-launch-national-health-information-exchange/. Accessed 10 January 2014. 
291. NaviNet. NaviNet HealthCare Communication Network Transactional Portal Information Exchange. Available from: http://ducknetweb.blogspot.co.uk/2009/08/navinet-healthcarecommunication.html. Accessed 15 February 2014.

292. Adler-Milstein J, Bates DW and Jha AK. Operational health information exchanges show substantial growth, but long-term funding remains a concern. Health Affairs. 2013;32(8):1486-92.

293. Medicine CoPSaHITlo. Health IT and Patient Safety: Building Safer Systems for Better Care. Institite of Medicine, Washington, DC: National Academies Press, 2012.

294. Lobach D. Proactive population health management within an HIE network: Duke Center for Health Informatics. Available from: https://www.dchi.duke.edu/about-us/dchi-book/Proactive $\% 20$ population $\% 20$ health $\% 20$ management $\% 20$ within $\% 20$ and $\% 20$ HIE\%20network.pdf. Accessed 20 January 2014.

295. Ancker JS, Edwards AM, Miller MC andKaushal R. Consumer perceptions of electronic health information exchange. American Journal of Preventive Medicine 2012;43(1):76-80.

296. Clisco. Cisco Medical Data Exchange Solution. Available from: http://www.cisco.com/web/strategy/healthcare/medical_data_ exchange_solution.html. Accessed 15 Februray 2014.

297. Dobbs D, Trebatoski M and Revere D. The Northwest Public Health Information Exchange's accomplishments in connecting a health information exchange with public health. Online Journal of Public Health Informatics 2010;2(2).

298. Downing GJ, Zuckerman AE, Coon C and Lloyd-Puryear MA, Ed. Enhancing the quality and efficiency of newborn screening programs through the use of health information technology. Seminars in Perinatology 2010;34(2):156-62.

299. L. Block P. Introduction to Health Informatics. Available from: http://www.philblock.info/hitkb/h/health_informatics.html.

300. MHiE. Physician Information Exchange. Available from: http://www.memorialhermann.org/healthcare-professionals/ physician-information-exchange/.

301. Minnesota. A Practical Guide to Understanding HIE, Assessing Your Readiness and Selecting HIE Options in Minnesota. Minnesota: Minnesota Department of Health.Available from: http://www.health.state.mn.us/divs/hpsc/ohit/hieguidance/intro. html\#hie.

302. Moore T, Shapiro JS, Doles L, Calman N, Camhi E, Check T et al., Ed. Event detection: a clinical notification service on a health information exchange platform. AMIA Annual Symposium Proceedings, 2012.

303. Mount_Sinai. Mount Sinai Health Information Exchange (HIE). Available from: http://www.mountsinai.org/ms-connect/mountsinai-health-information-exchange-hie. Accessed 21 December 2013.

304. Magnus M, Herwehe J, Andrews L, Gibson L, Daigrepont N, De Leon JM et al. Evaluating health information technology: provider satisfaction with an HIV-specific, electronic clinical management and reporting system. AIDS Patient Care and STDs 2009;23(2):85-91.

305. Nakamura MM, Ferris TG, DesRoches CM and Jha AK. Electronic health record adoption by children's hospitals in the United States. Archives of pediatrics and Adolescent Medicine 2010;164(12): 1145 .

306. Ozkaynak M and Brennan PF. Revisiting sociotechnical systems in a case of unreported use of health information exchange system in three hospital emergency departments. Journal of Evaluation in Clinical Practice 2013;19(2):370-3.

307. Tripathi M, Delano D, Lund B and Rudolph L. Engaging patients for health information exchange. Health Affairs 2009;28(2):435-43.

308. Virginia C. Welcome to Virginia's Statewide Health Information Exchange (HIE). Available from: https://www.connectvirginia. org/. Accessed 20 January 2014.

309. Xerox. Healthcare Information Exchange (HIE). Available from: http://services.xerox.com/informed-health/healthcare-itsolutions/health-information-exchange/enus.html. Accessed 20 January 2014. 
$\stackrel{\Re}{\wedge}$

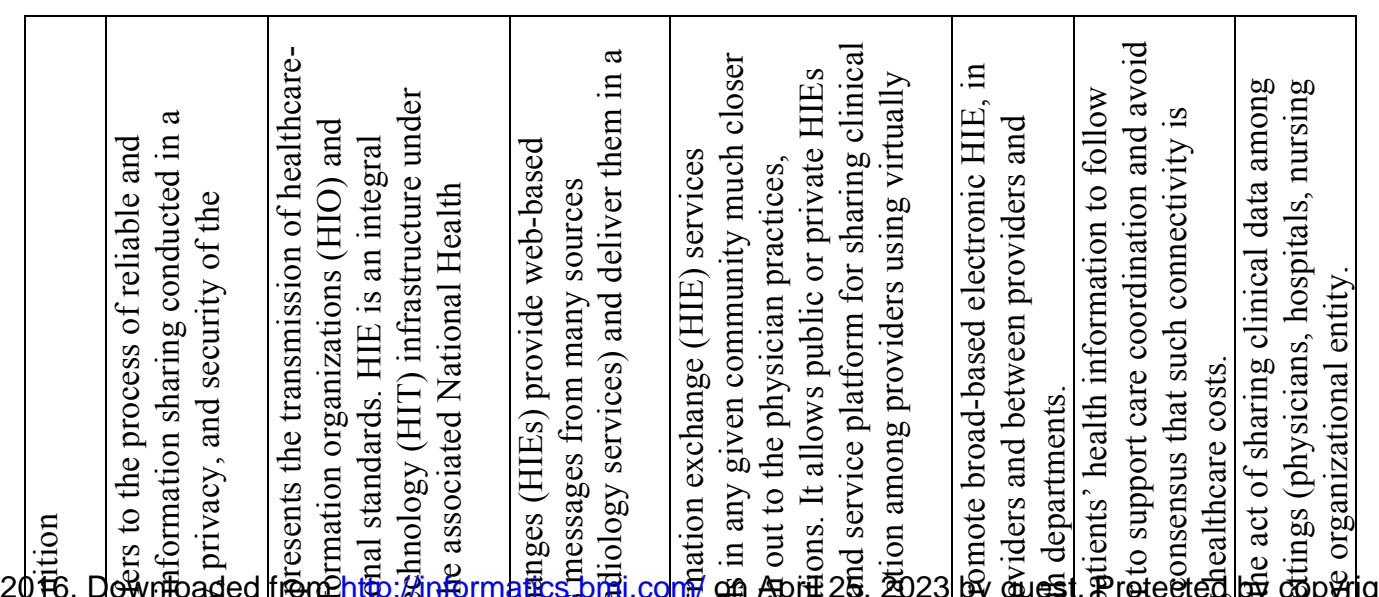
4236/jhi.v23i4.838 on 1 October 20.

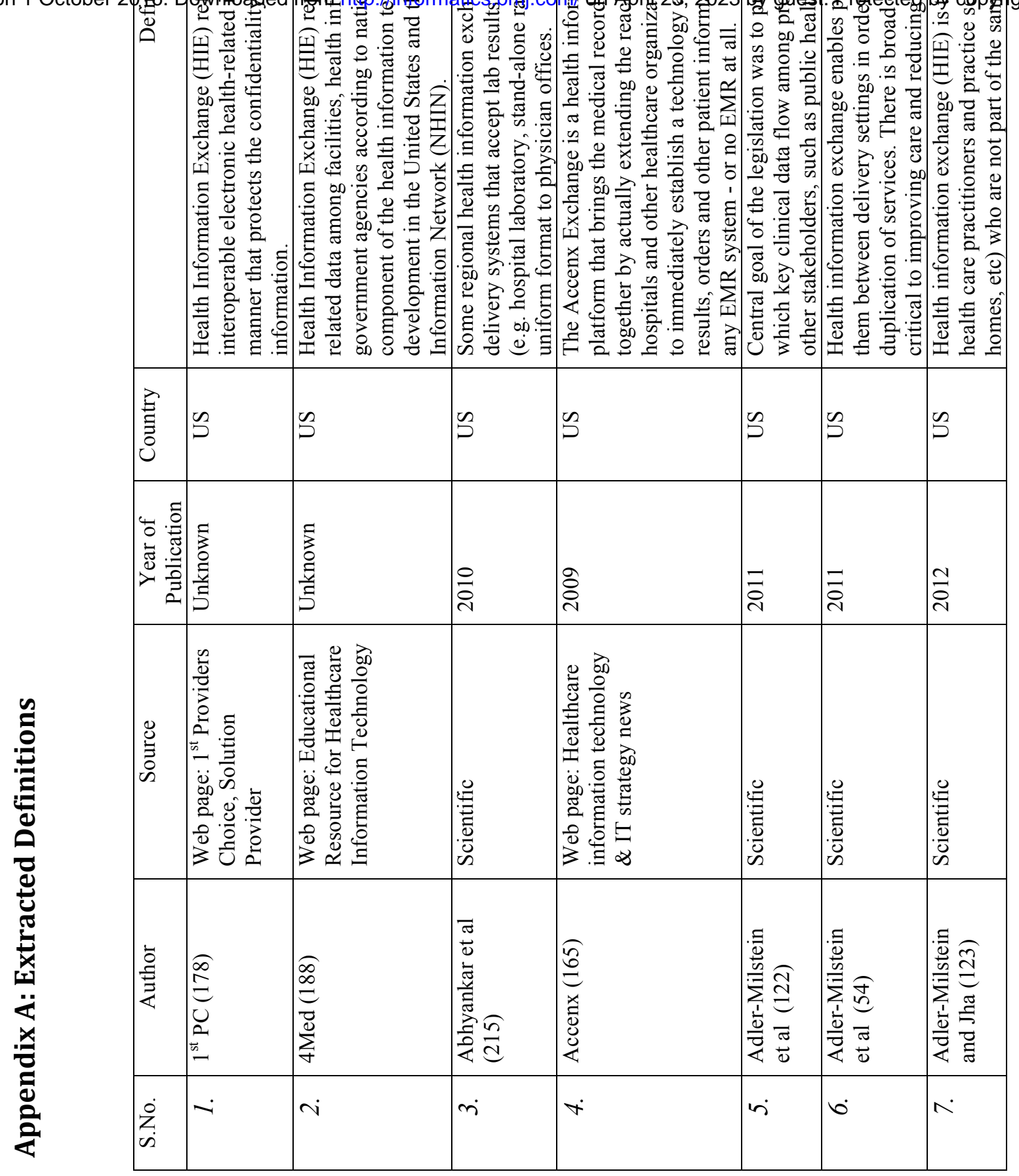


$\stackrel{+}{~}$

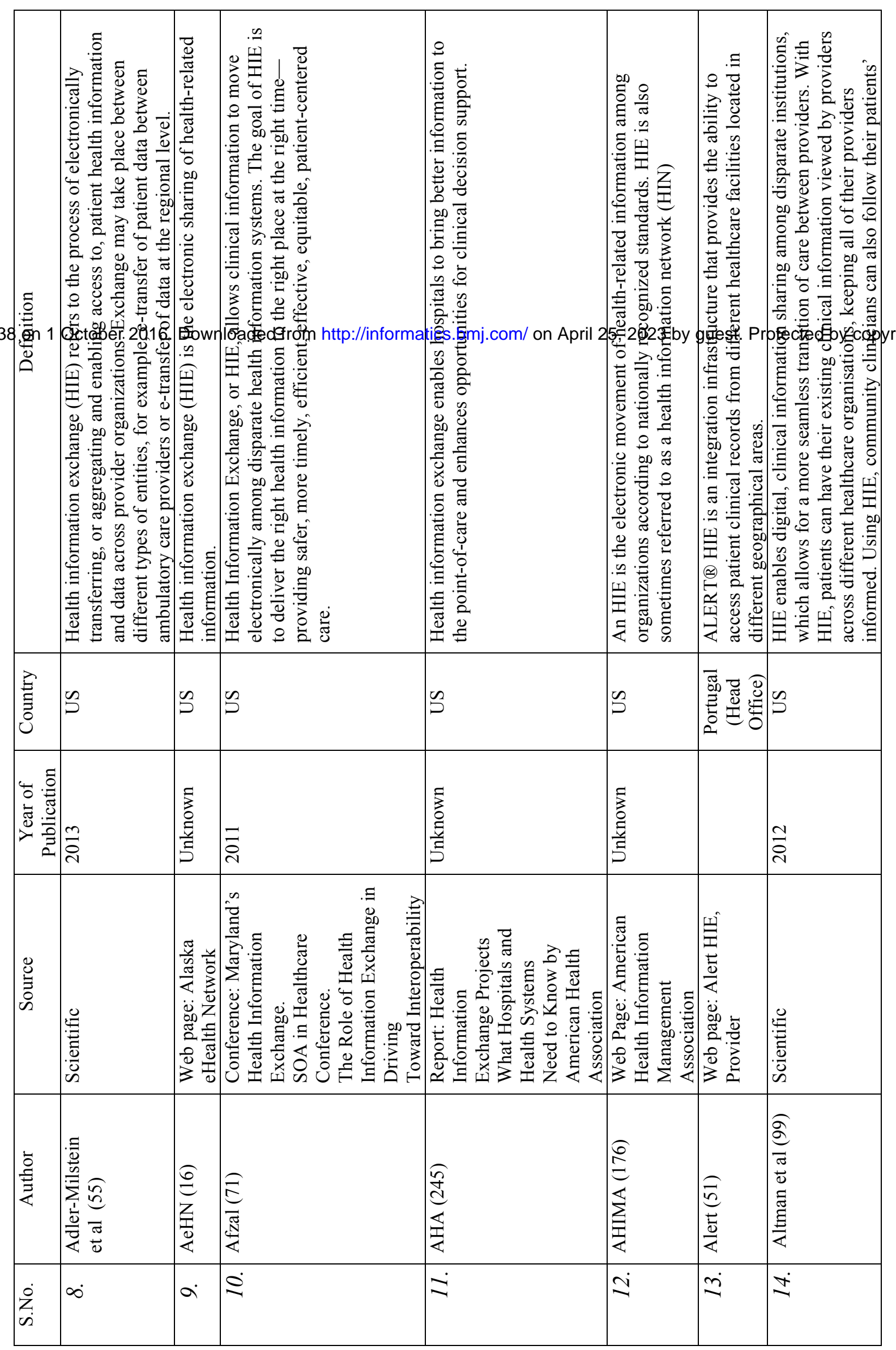




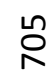

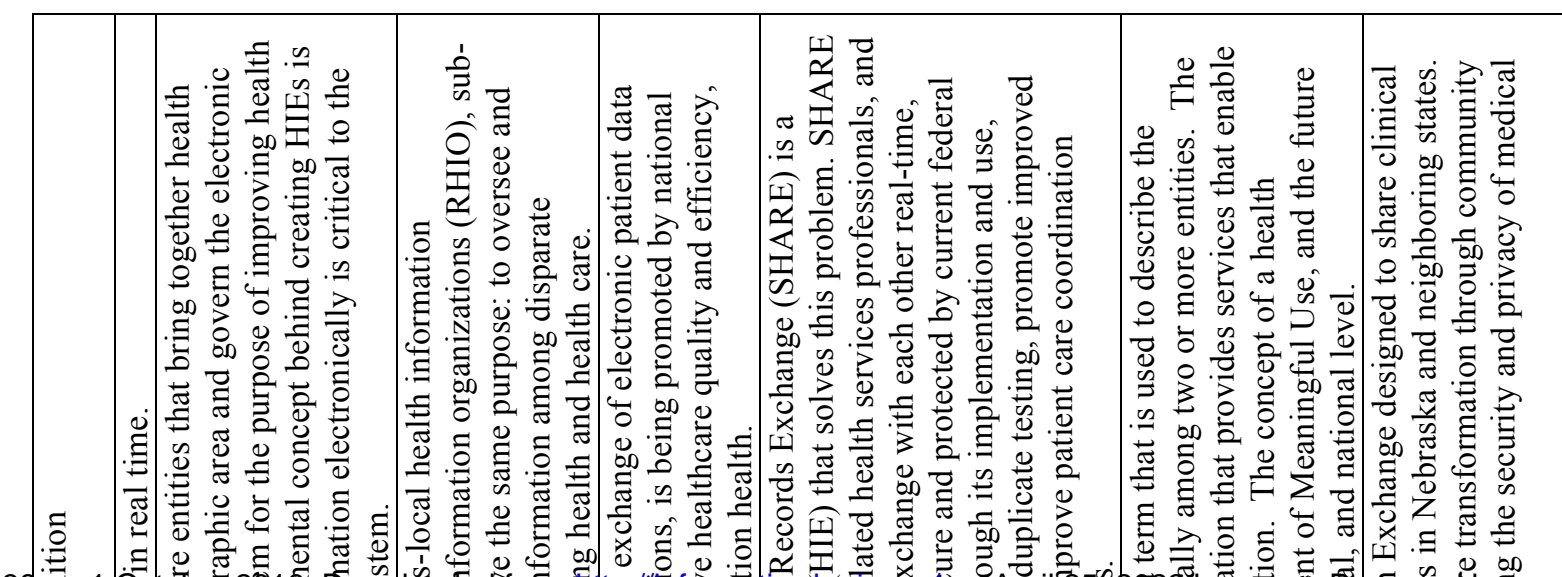

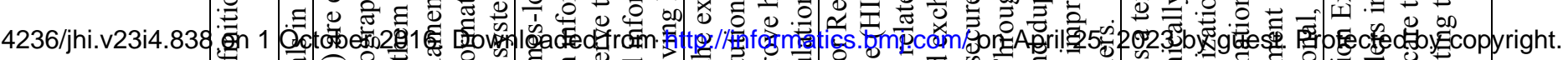

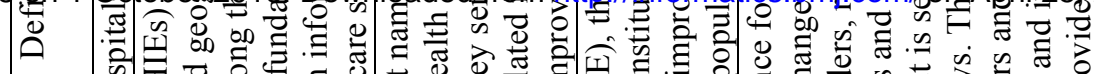

.

\begin{tabular}{|c|c|c|c|c|c|c|}
\hline & 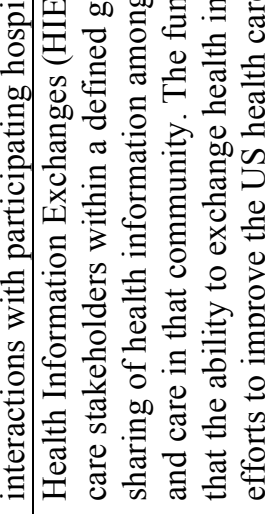 & 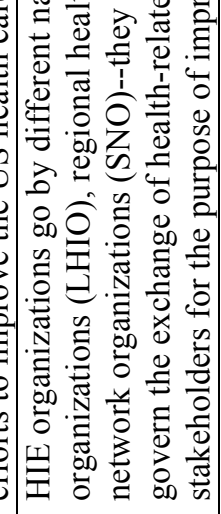 & 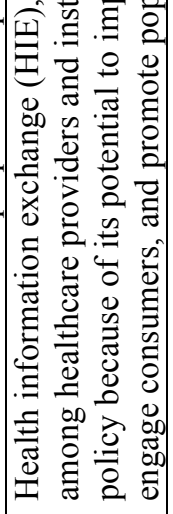 & 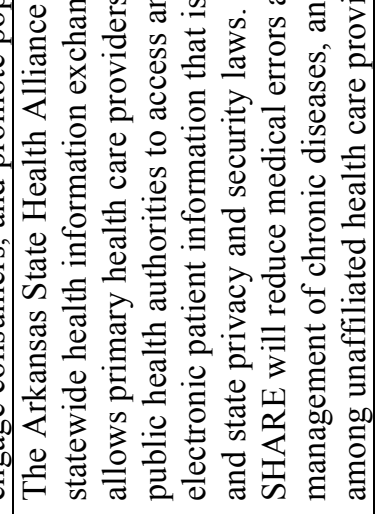 & 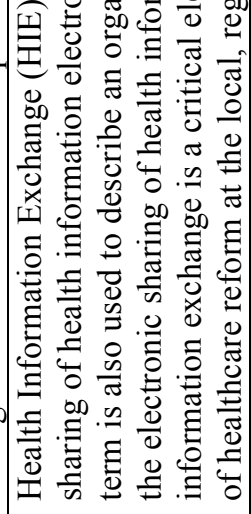 & 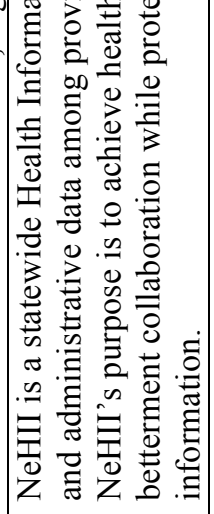 \\
\hline $\begin{array}{l}\vec{E} \\
\stackrel{\Xi}{\Xi} \\
0\end{array}$ & $\tilde{2}$ & 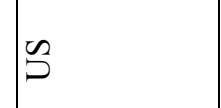 & 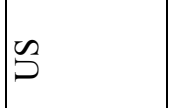 & 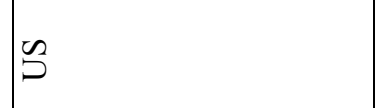 & $\Omega$ & $\Omega$ \\
\hline 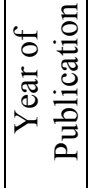 & 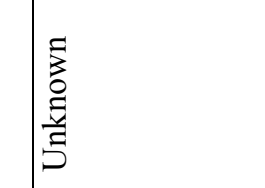 & $\stackrel{\infty}{\stackrel{\aleph}{े}}$ & $\frac{\sim}{\stackrel{\sim}{\sim}}$ & 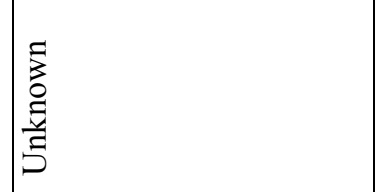 & 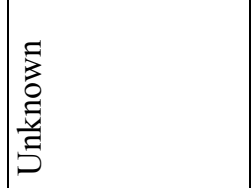 & ஓे \\
\hline 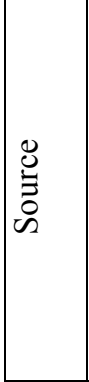 & 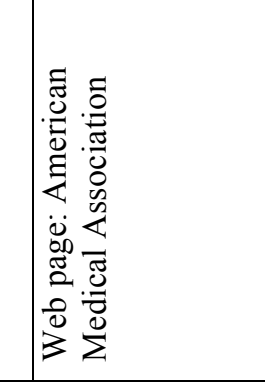 & 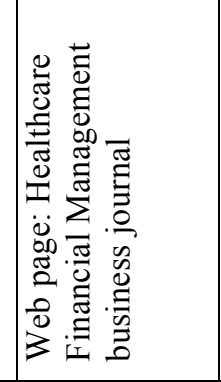 & 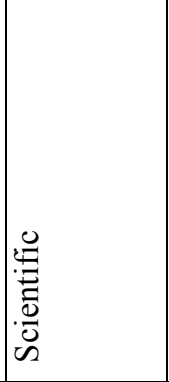 & 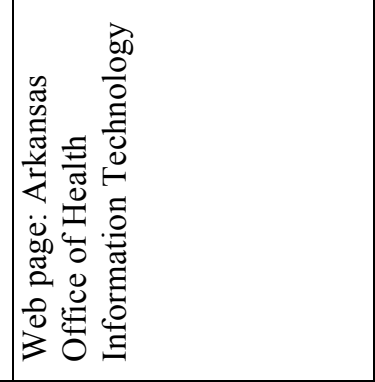 & 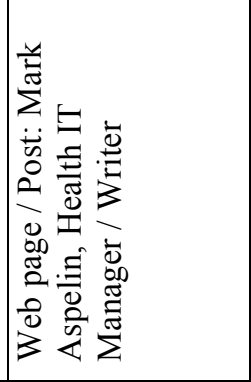 & 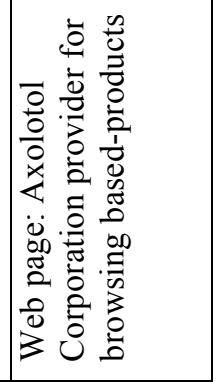 \\
\hline 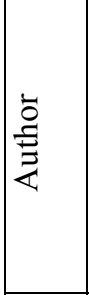 & 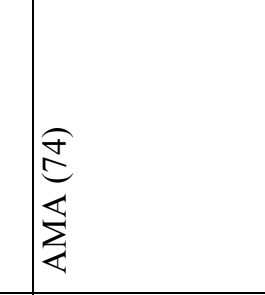 & 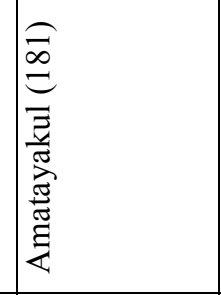 & 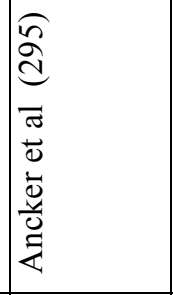 & 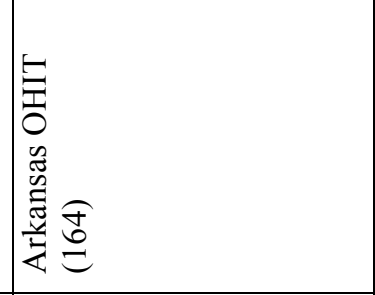 & 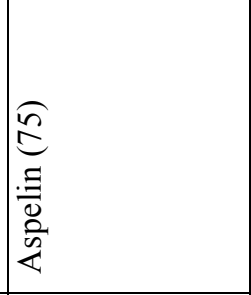 & 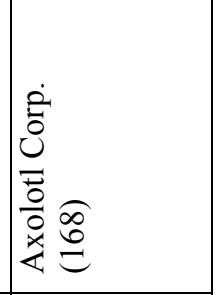 \\
\hline$\dot{8}$ & $\stackrel{2}{=}$ & $\stackrel{0}{=}$ & $\triangleq$ & $\infty$ & 2 & $\dot{\curvearrowright}$ \\
\hline
\end{tabular}


$\stackrel{8}{\circ}$

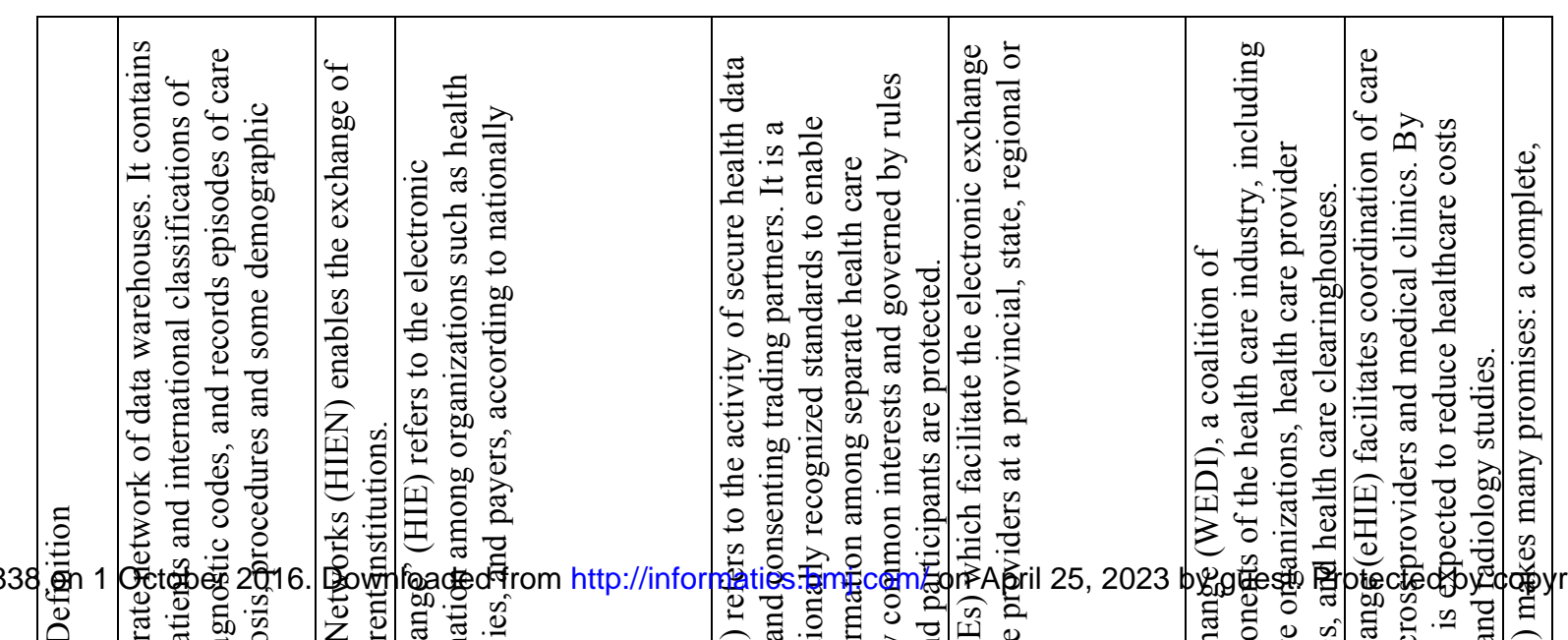

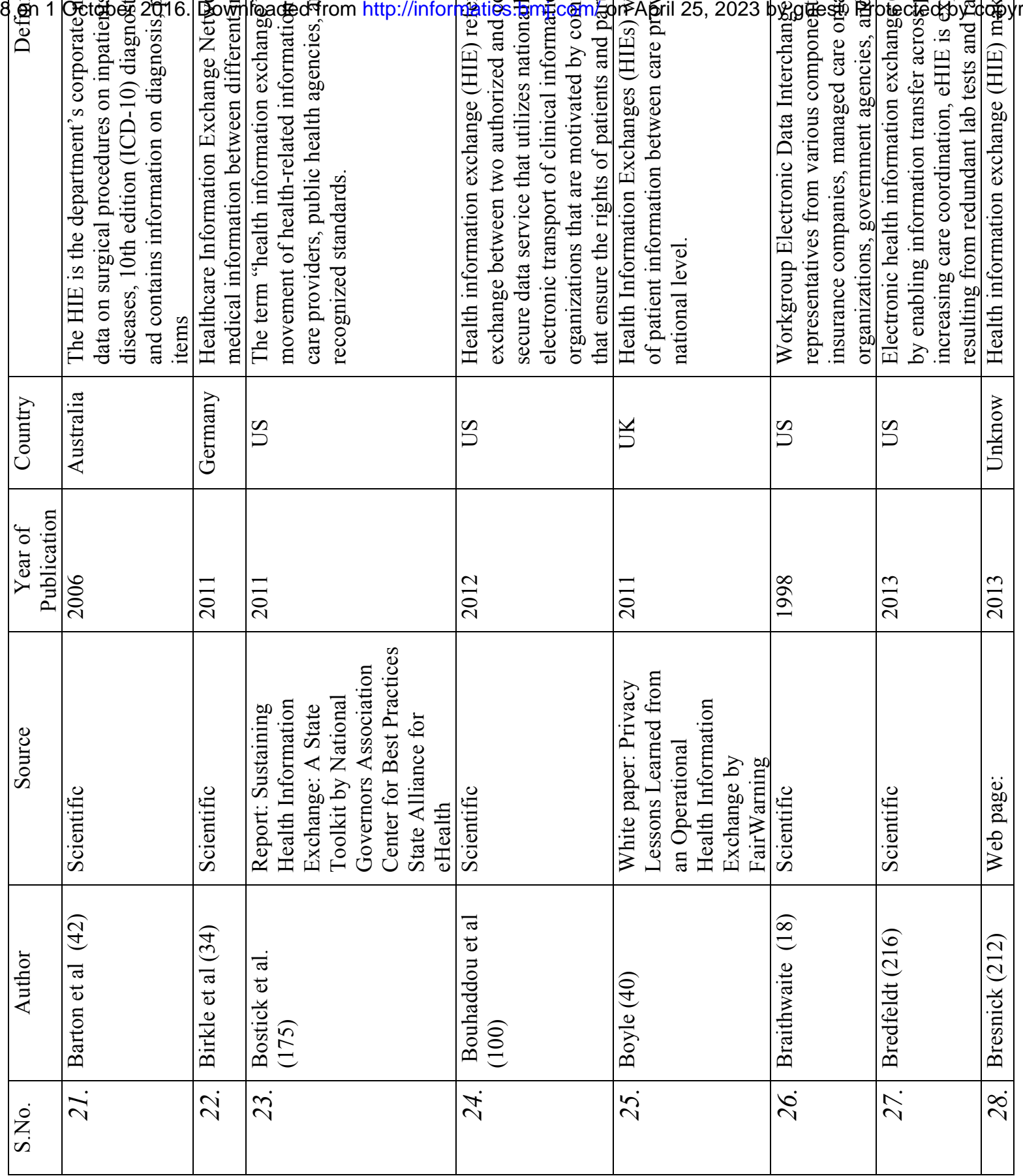


$\hat{2}$

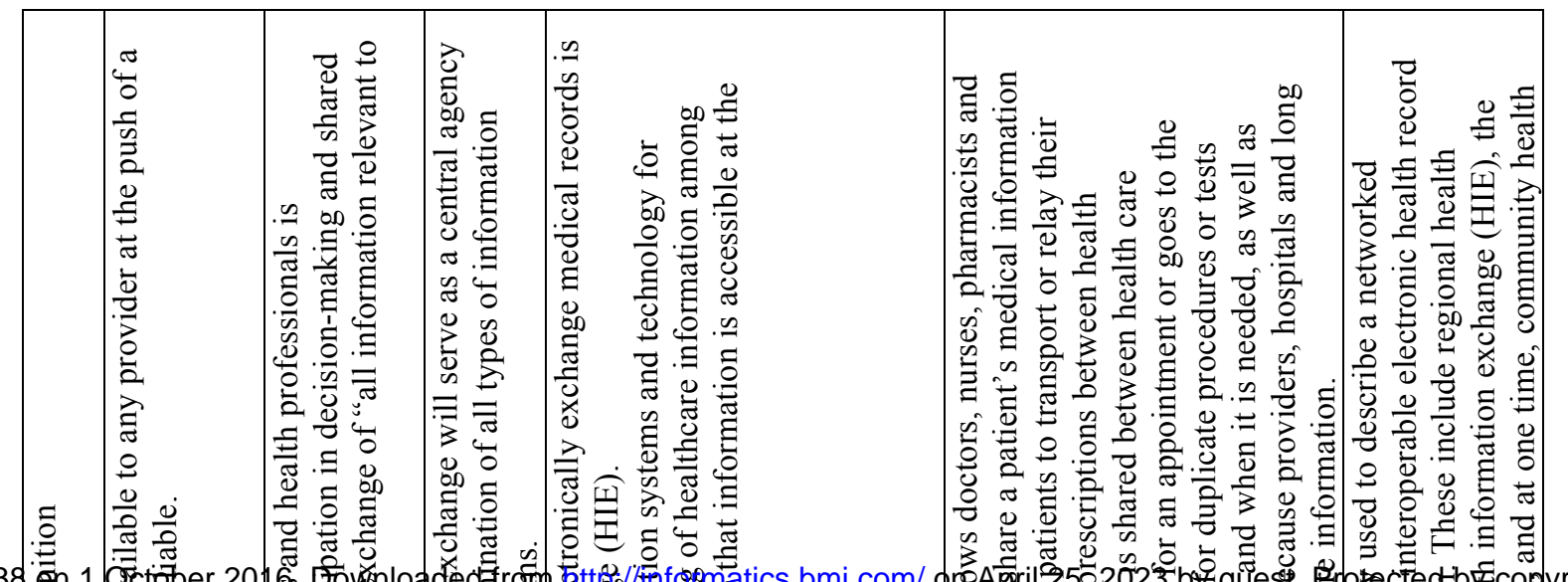

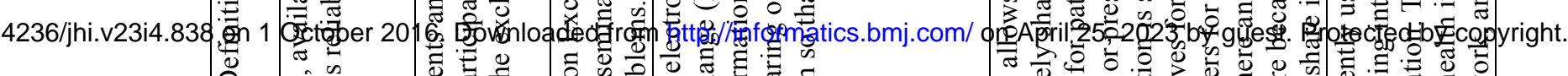

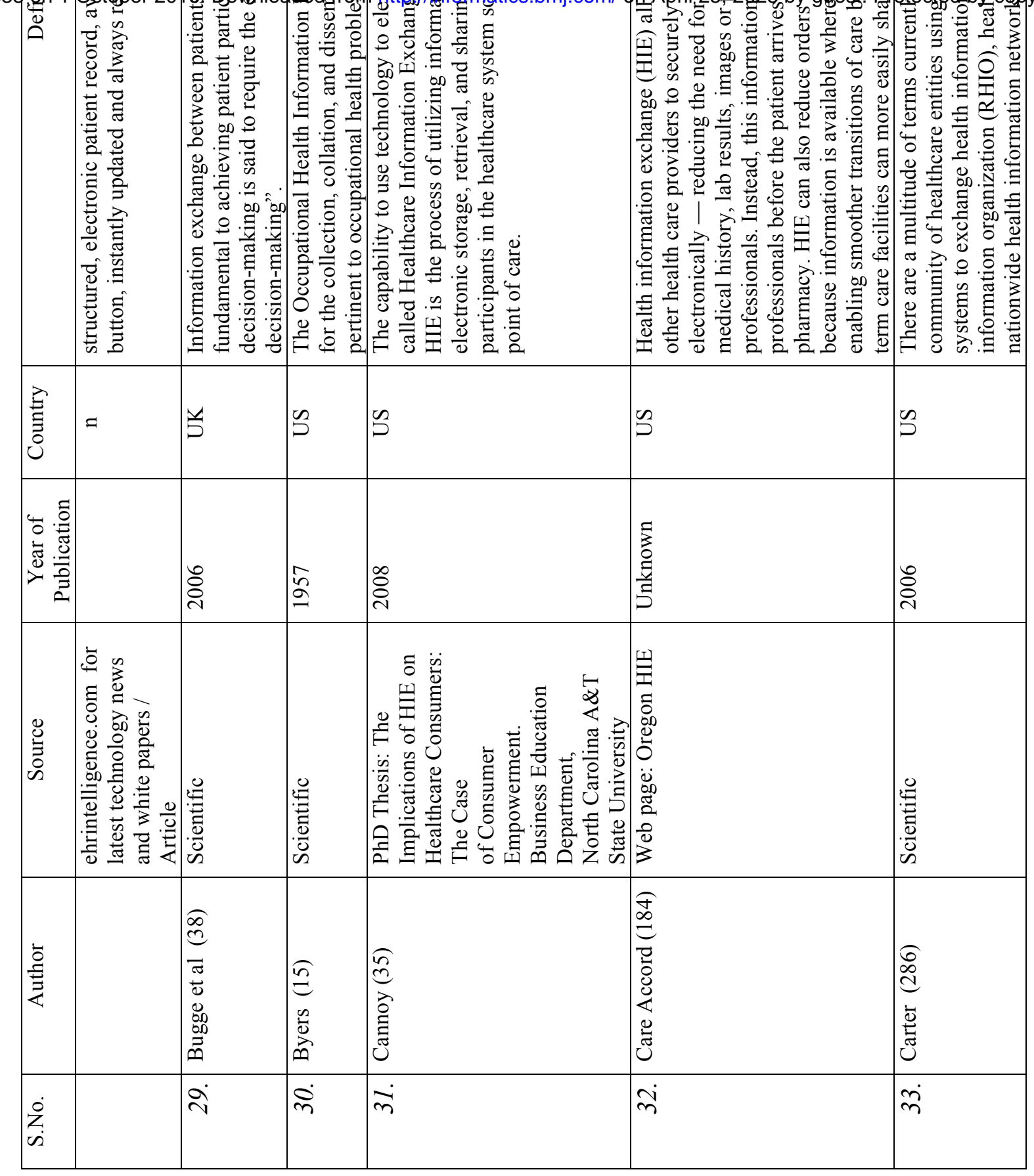


$\stackrel{\infty}{\wedge}$

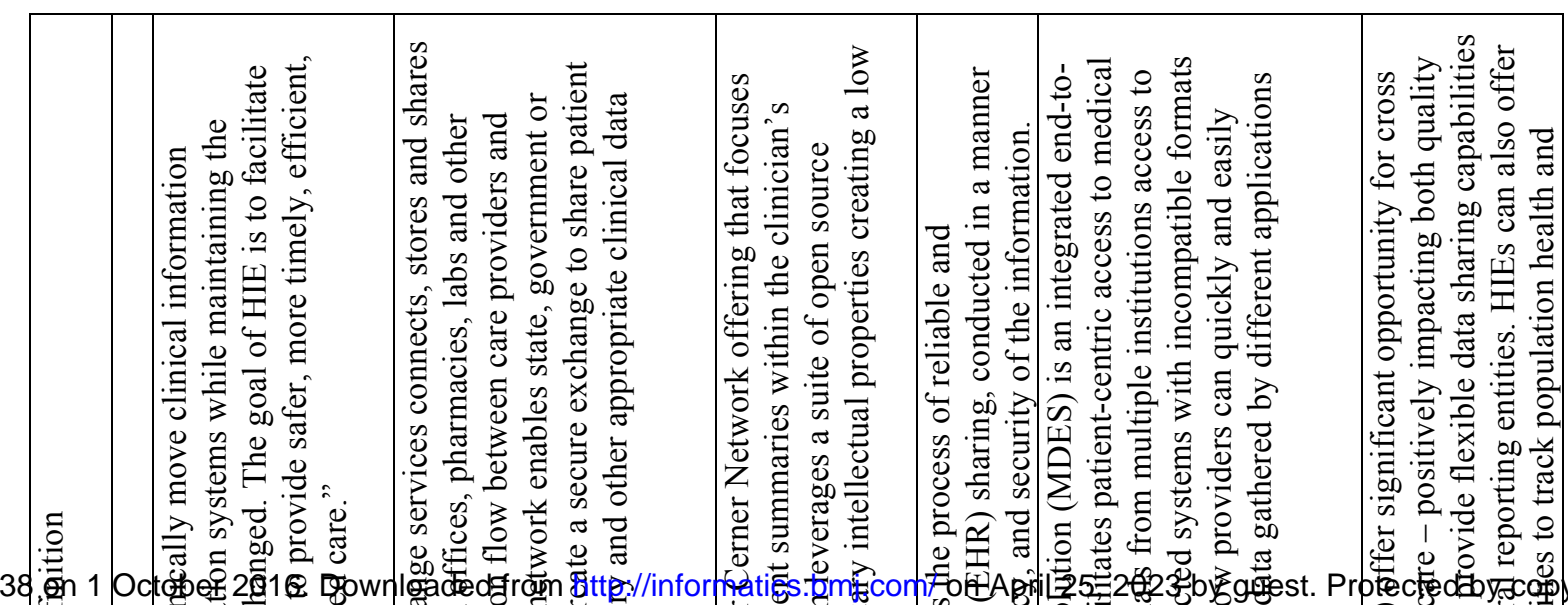

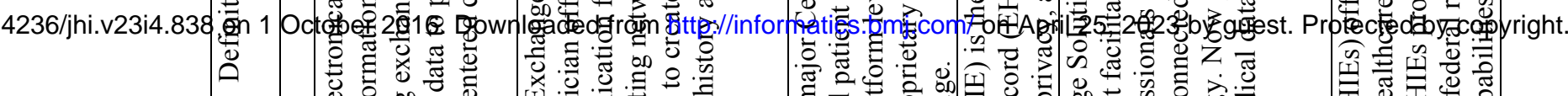

\begin{tabular}{|c|c|c|c|c|c|c|}
\hline ๑ొ & 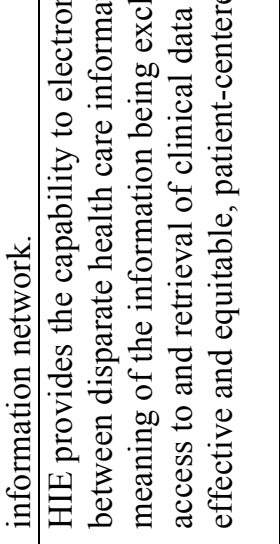 & 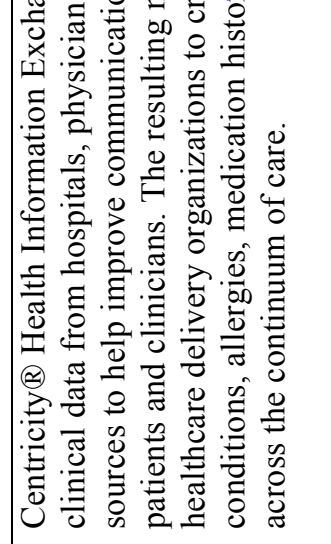 & 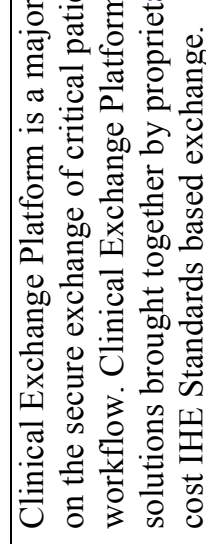 & 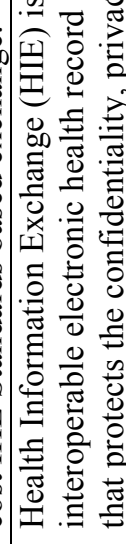 & 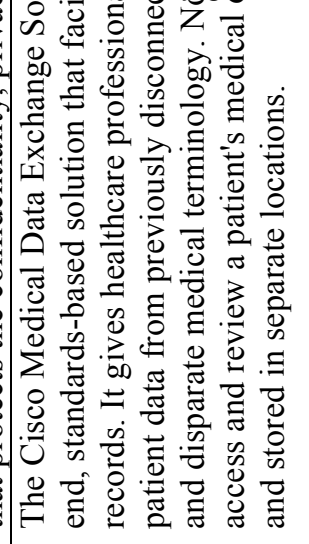 & 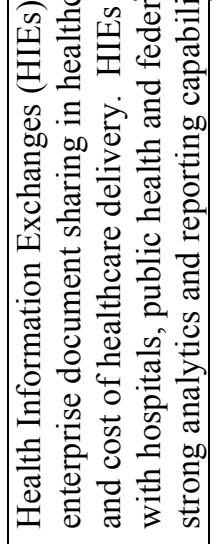 \\
\hline $\begin{array}{l}\text { E } \\
\text { 音 }\end{array}$ & 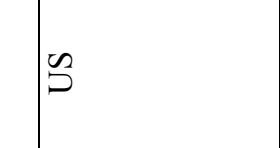 & $\Omega$ & $\tilde{\Omega}$ & $\Omega$ & $\Omega$ & $\tilde{s}$ \\
\hline 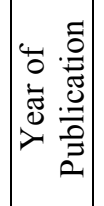 & 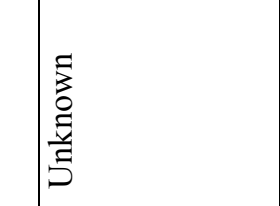 & ஓे & 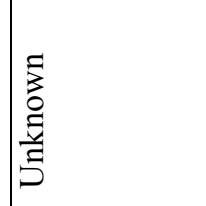 & $\frac{\sim}{\stackrel{\sim}{\sim}}$ & 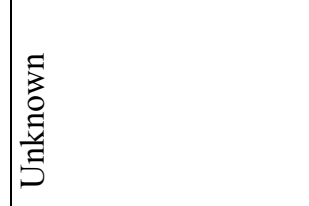 & 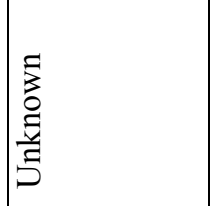 \\
\hline 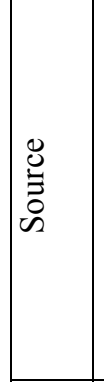 & 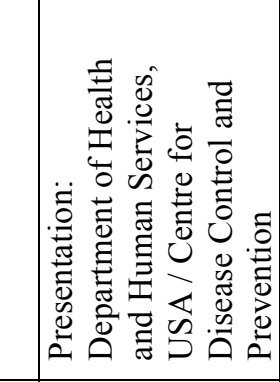 & 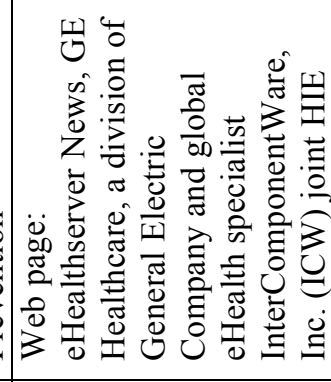 & 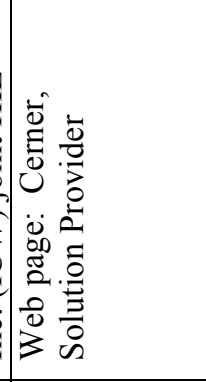 & 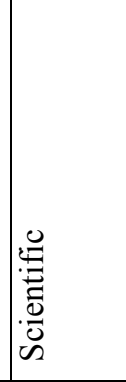 & 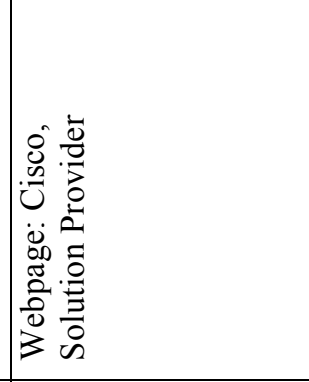 & 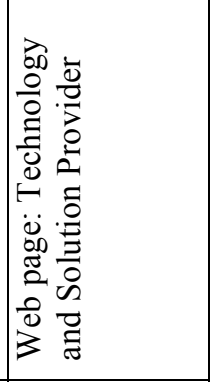 \\
\hline 总 & $\begin{array}{l}\underset{\Xi}{\Xi} \\
己 \\
0 \\
0\end{array}$ & 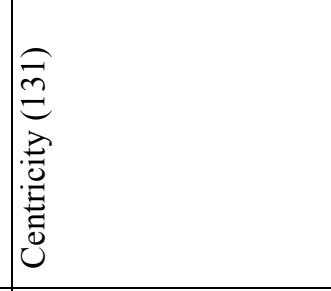 & 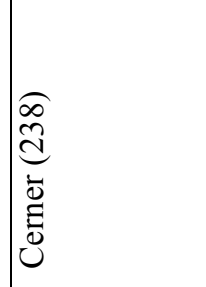 & 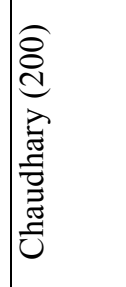 & 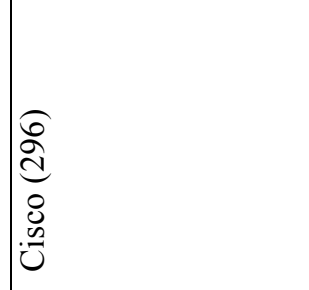 & 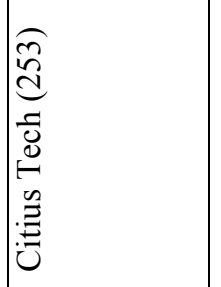 \\
\hline $\begin{array}{l}\dot{z} \\
\dot{m}\end{array}$ & $\ddot{m}$ & $\dot{m}$ & $\ddot{n}$ & $\hat{n}$ & $\infty$ & $\dot{m}$ \\
\hline
\end{tabular}


$\stackrel{8}{2}$

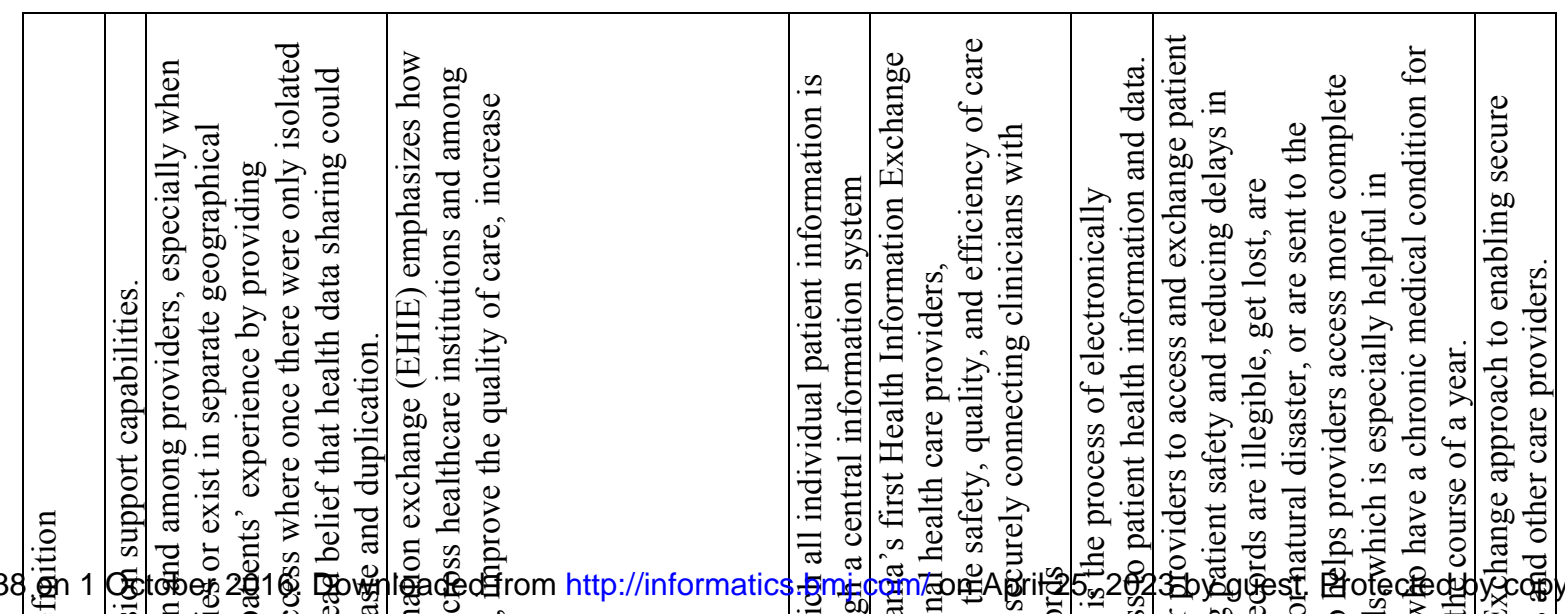

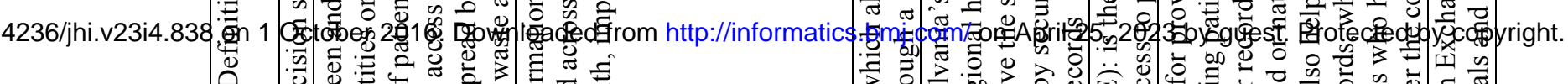

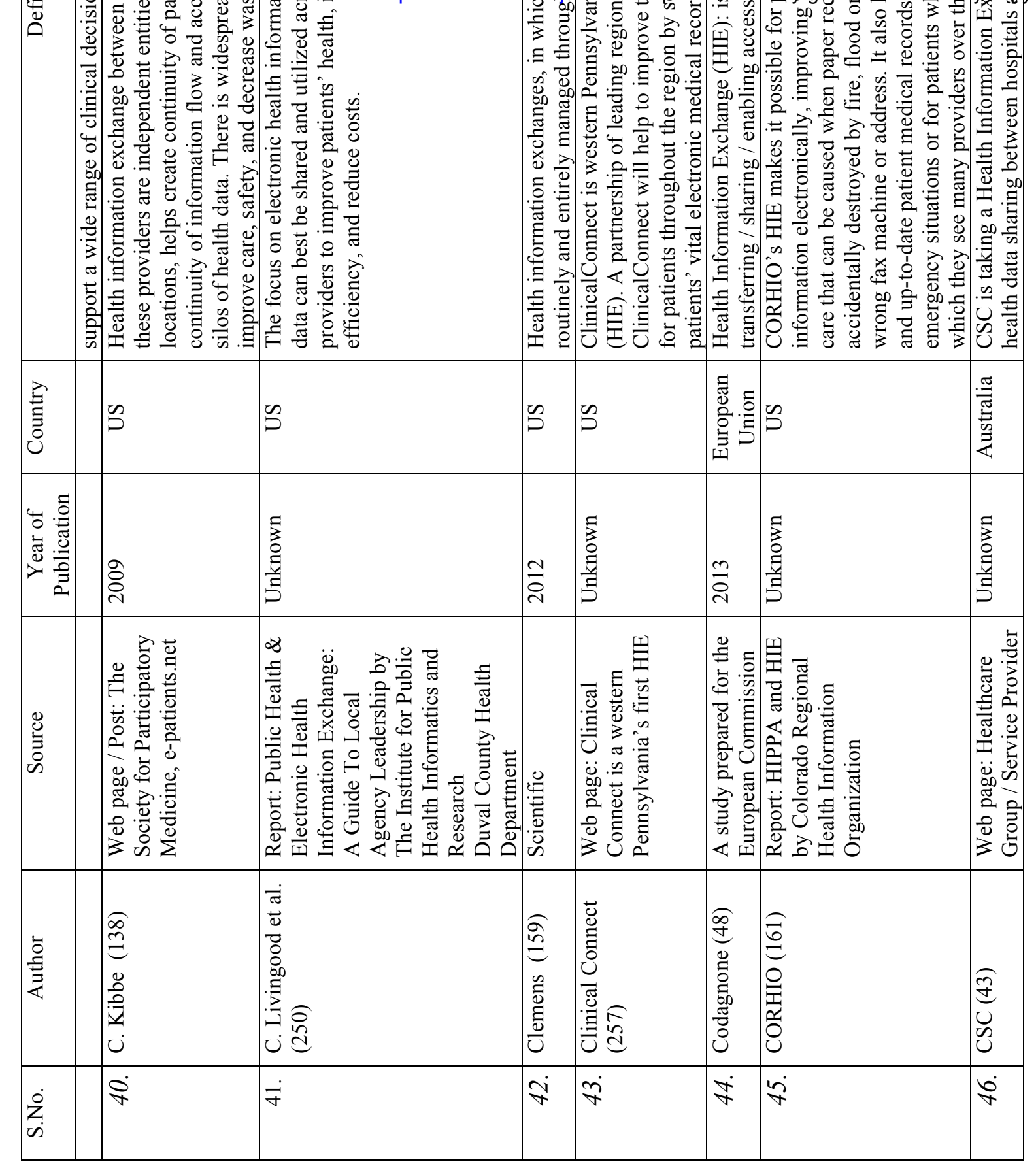


$\stackrel{9}{\wedge}$
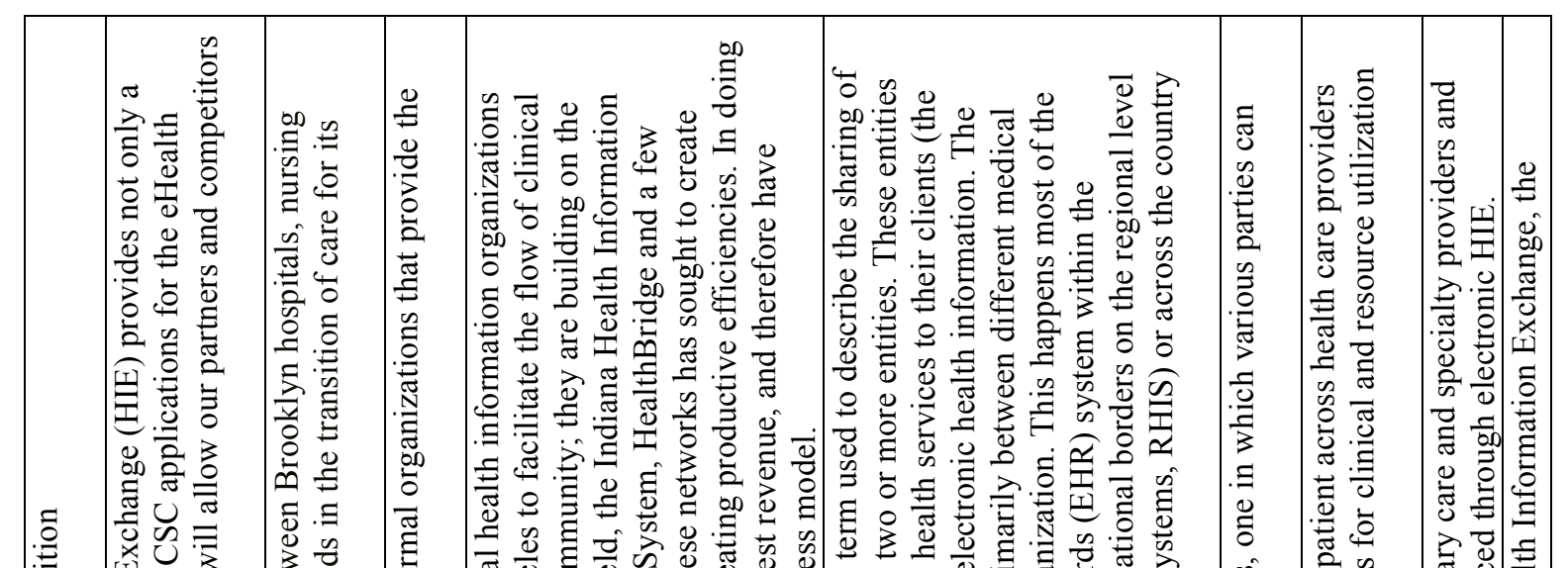

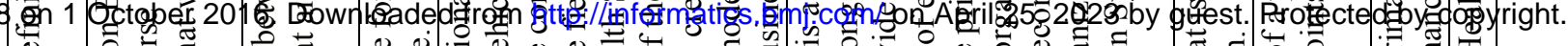

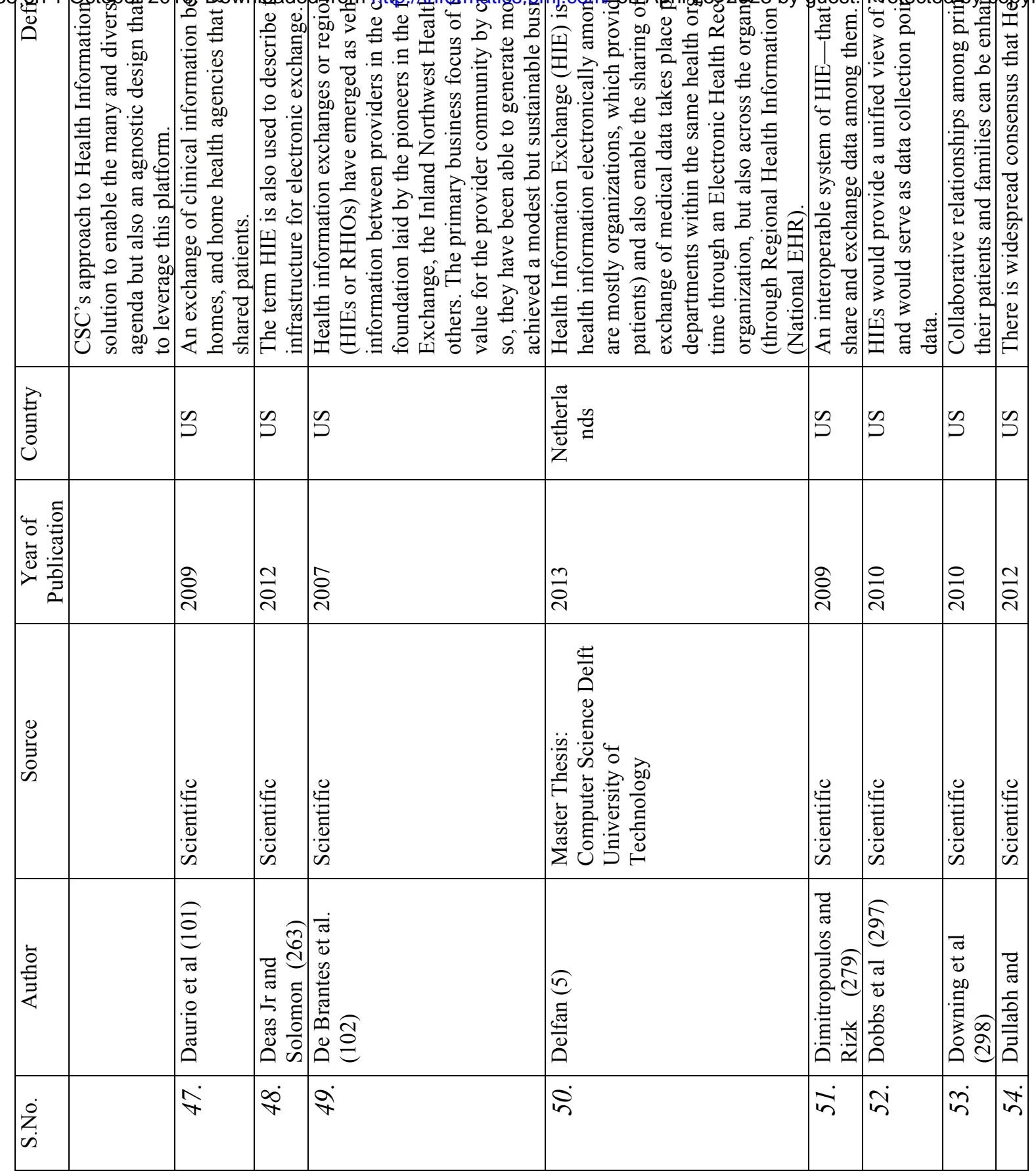


곡

\begin{tabular}{|c|c|c|c|c|}
\hline$\stackrel{\varrho}{\varrho}$ & 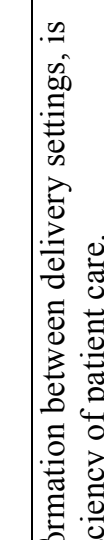 & 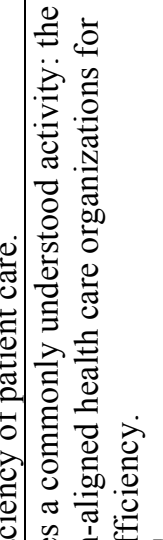 & 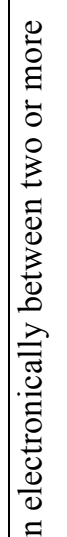 & 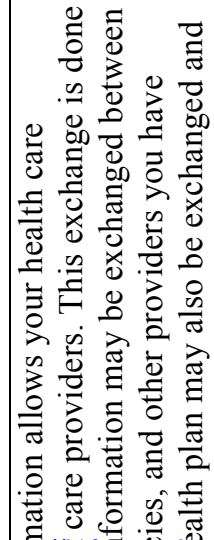 \\
\hline
\end{tabular}

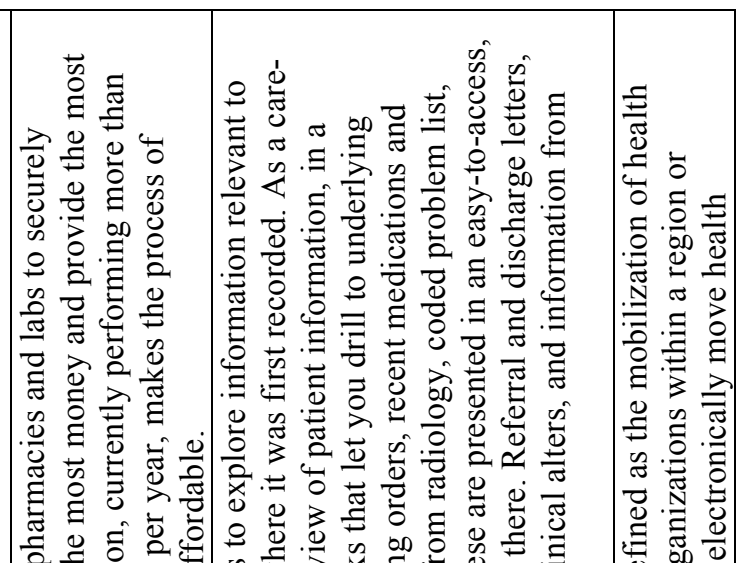

\begin{tabular}{|c|c|c|c|c|c|c|c|}
\hline 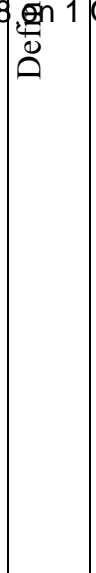 & 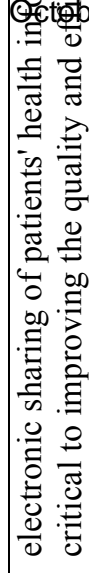 & 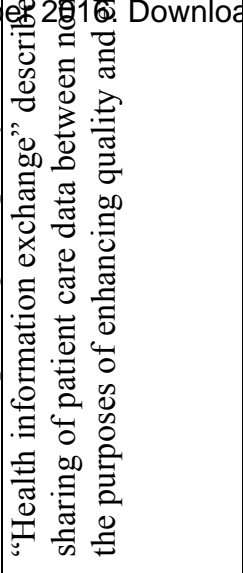 & 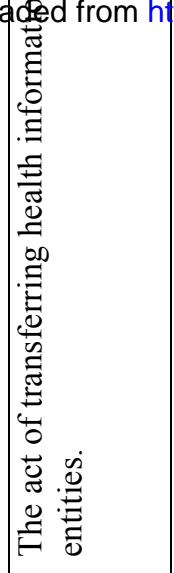 & 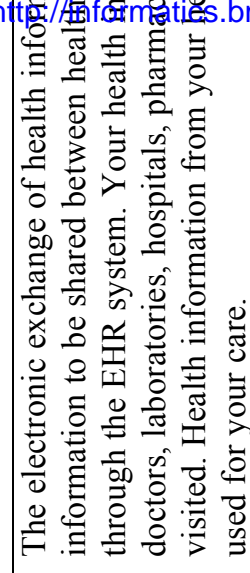 & 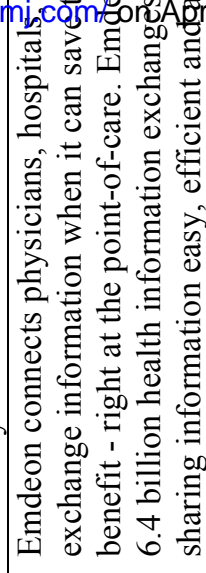 & 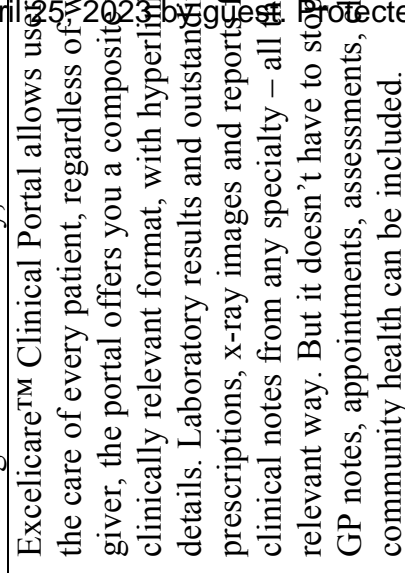 & 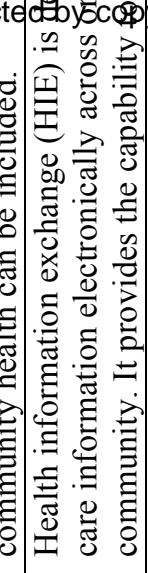 \\
\hline 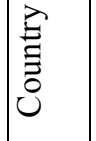 & & 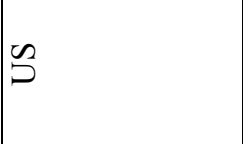 & $\Omega$ & $\tilde{\Omega}$ & $\Omega$ & $\underline{J}$ & $\tilde{\Omega}$ \\
\hline 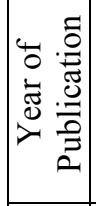 & & సे & $\overline{\bar{\sigma}}$ & $\overrightarrow{\vec{i}}$ & 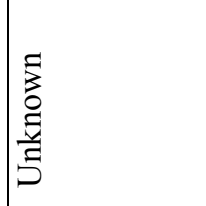 & 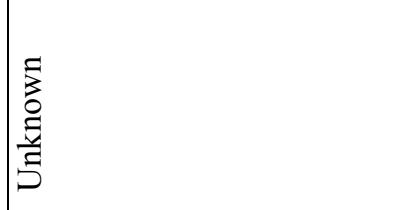 & $\overline{\vec{N}}$ \\
\hline 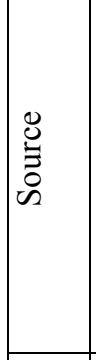 & & 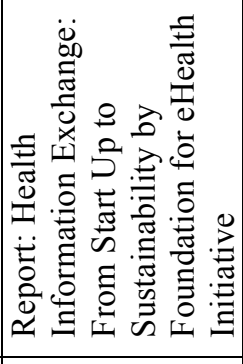 & 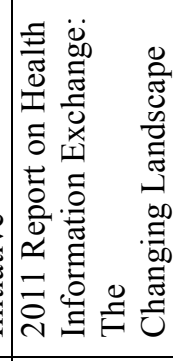 & 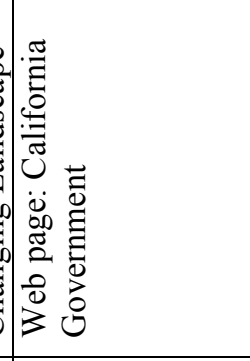 & 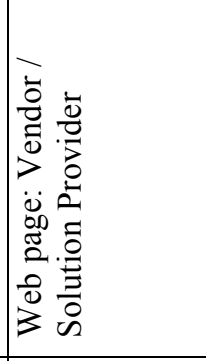 & 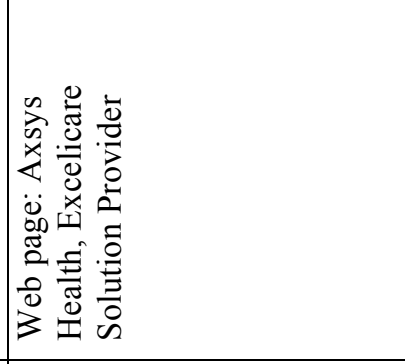 & 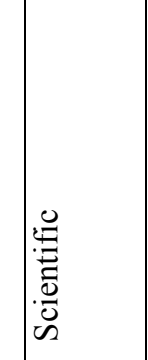 \\
\hline 总 & 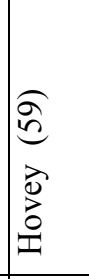 & 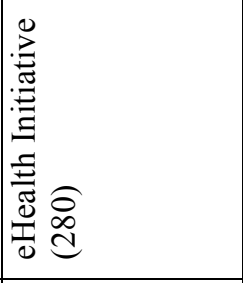 & 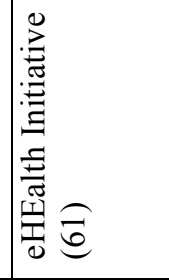 & 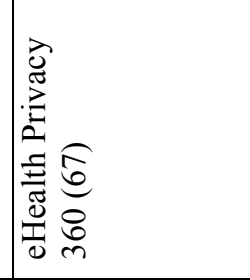 & 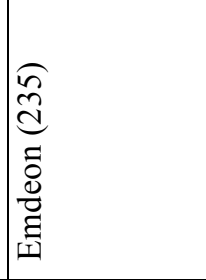 & 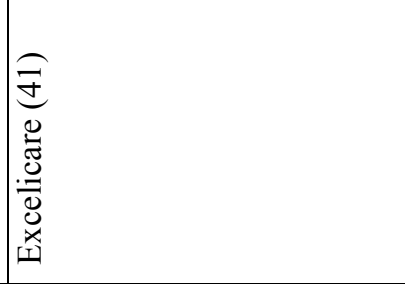 & 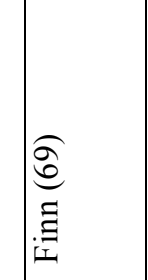 \\
\hline$\ddot{z}$ & & in & $b^{\circ}$ & in & in & in & 6 \\
\hline
\end{tabular}


กี

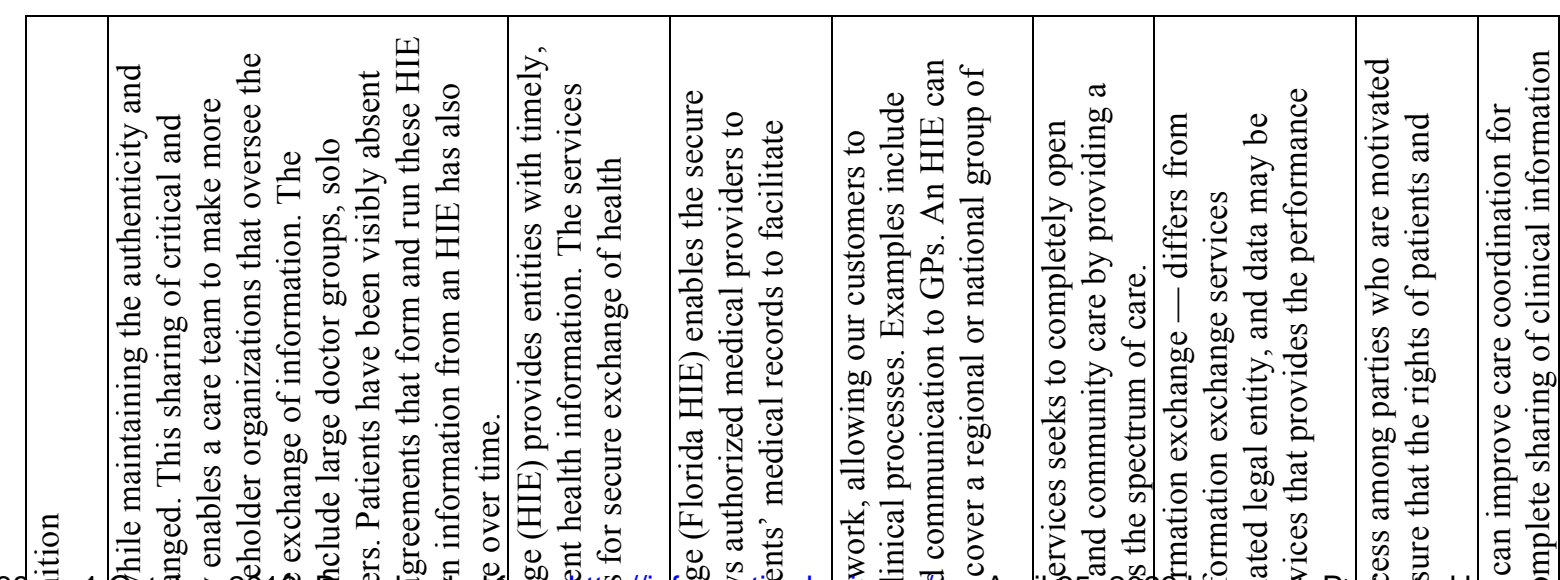

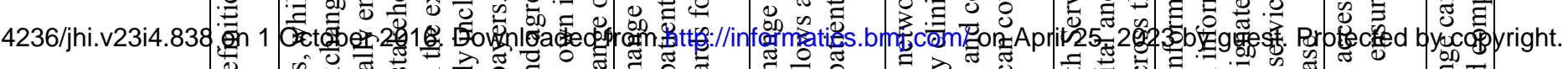

它

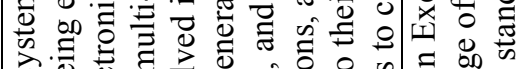

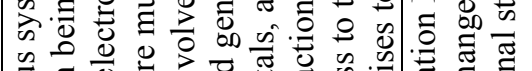

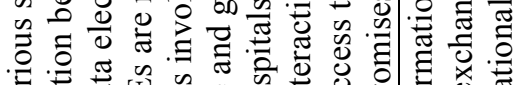

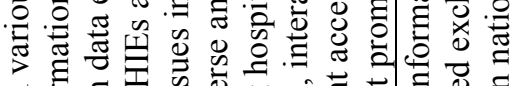

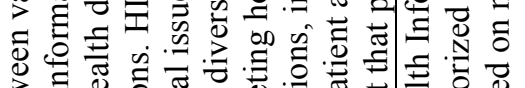

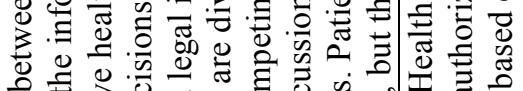

\begin{tabular}{|c|c|c|c|c|c|c|c|}
\hline 䚻 & $s$ & $s$ & 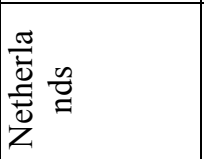 & 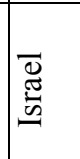 & $s$ & 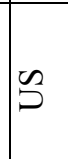 & 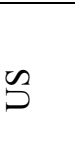 \\
\hline 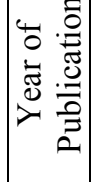 & $\begin{array}{l}\text { 咅 } \\
\text { 咅 } \\
\end{array}$ & 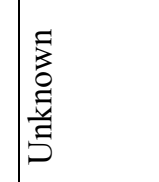 & 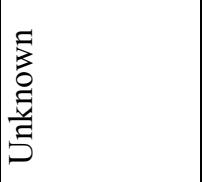 & ते & 商 & $\frac{\bar{a}}{2}$ & 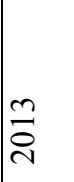 \\
\hline |亏 & 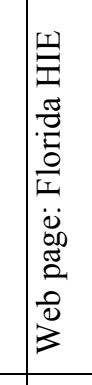 & 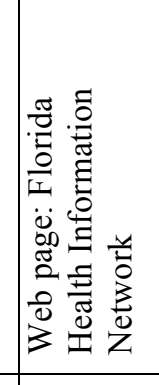 & 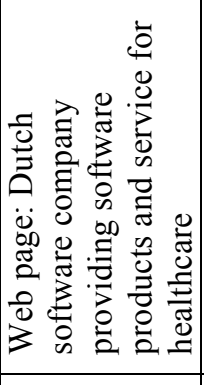 & 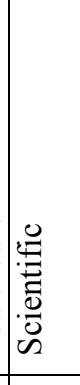 & 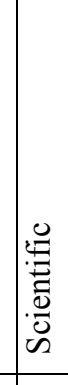 & 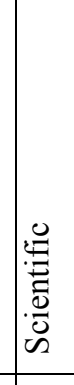 & 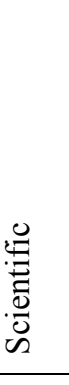 \\
\hline 竞 & 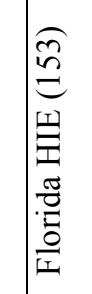 & 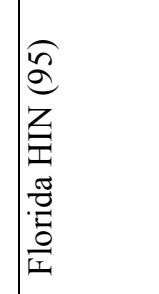 & 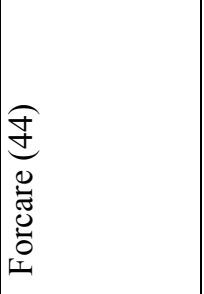 & 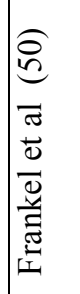 & 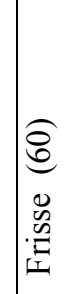 & 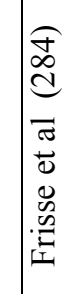 & 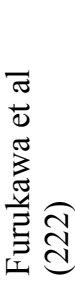 \\
\hline ì & $\dot{\sigma}$ & ชi & ஜ் & I8 & 16 & 80 & 8 \\
\hline
\end{tabular}


$\stackrel{m}{N}$

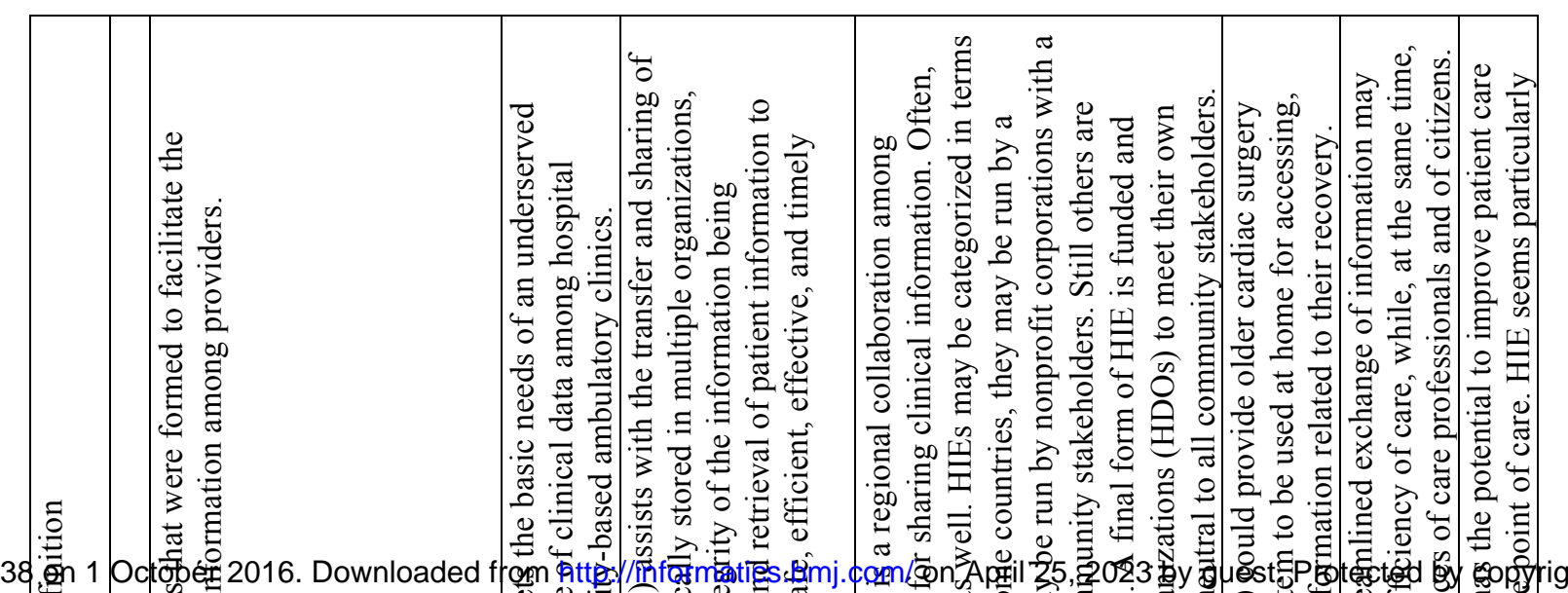

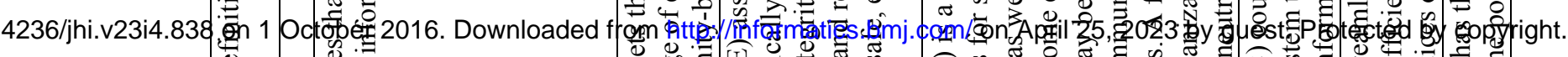

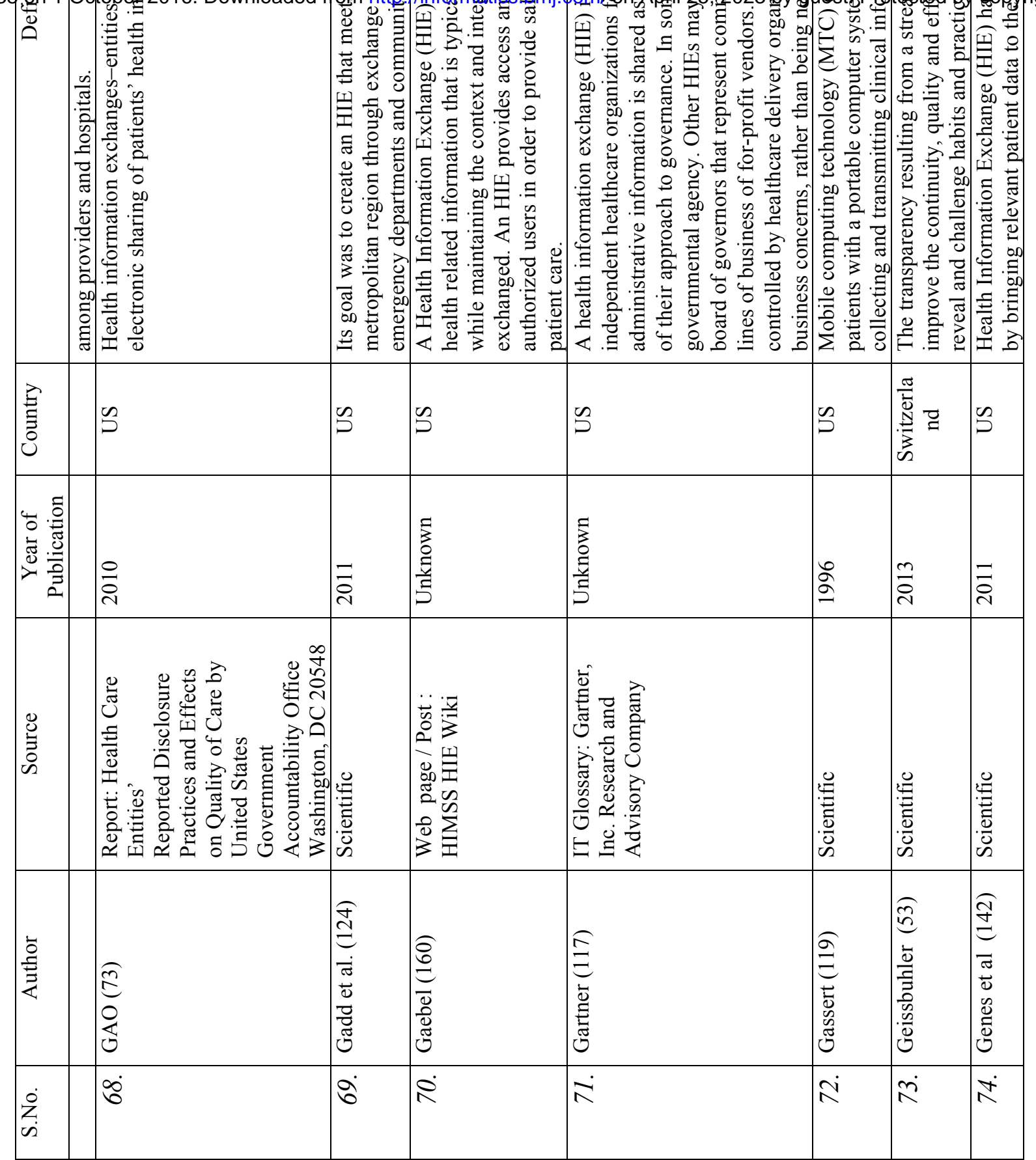


্ㅠ

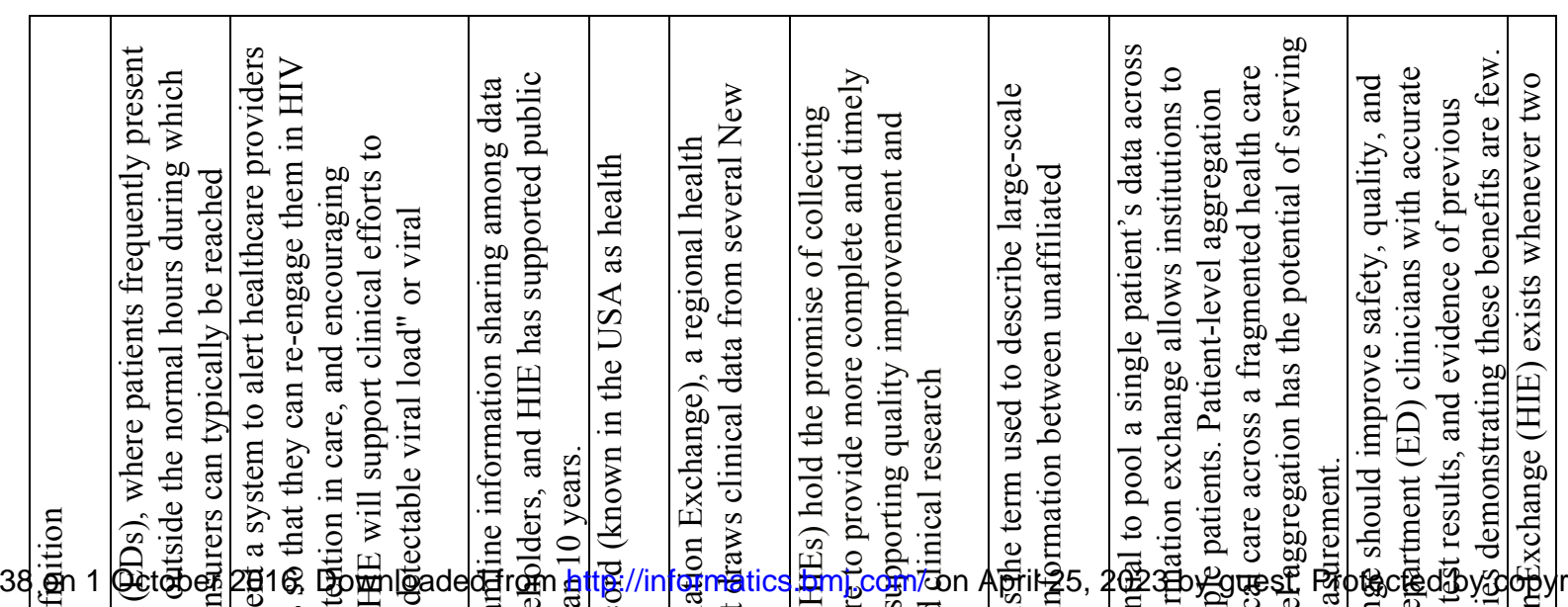

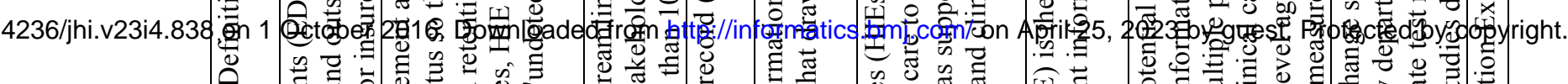

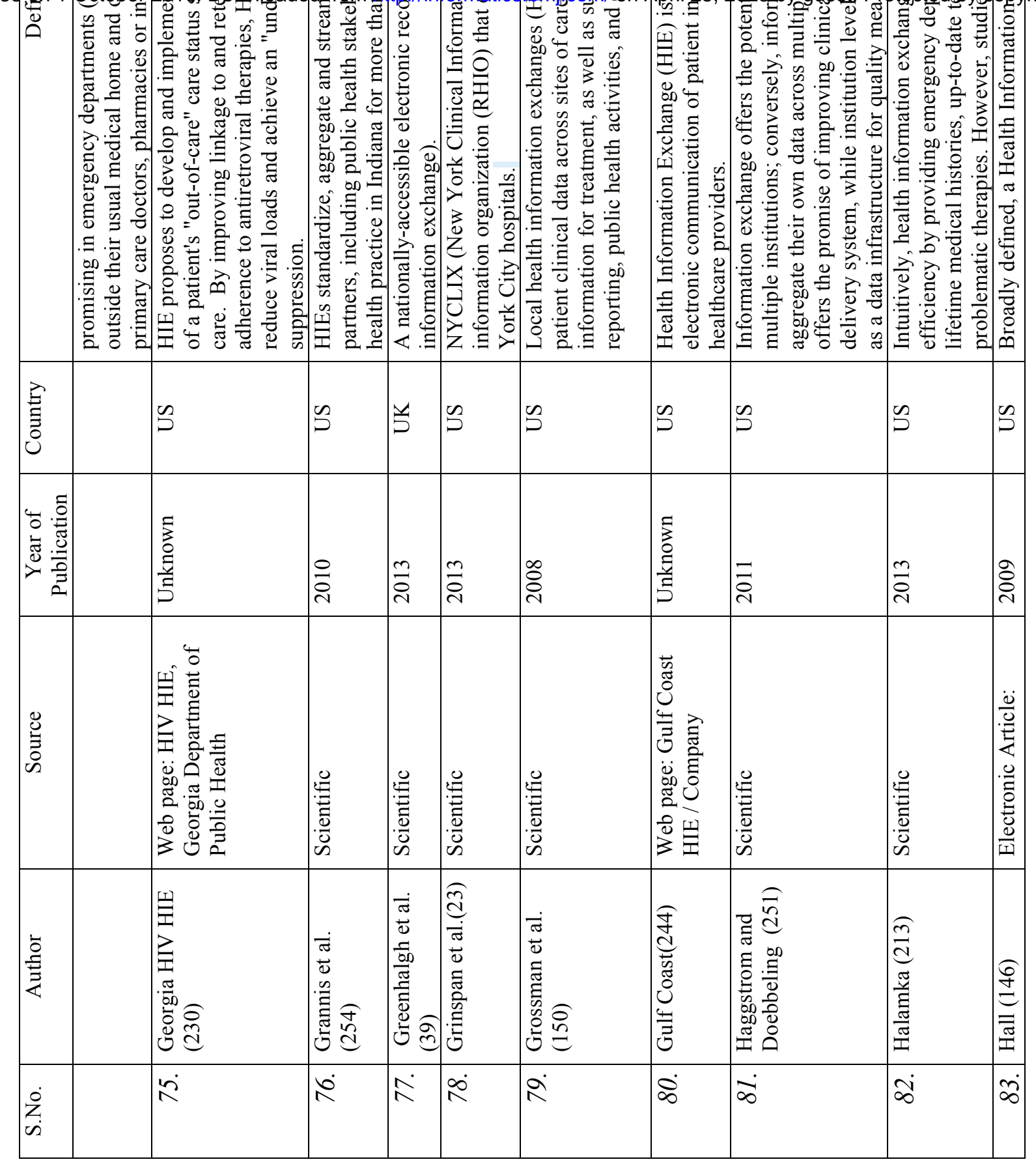


$\stackrel{n}{\Lambda}$

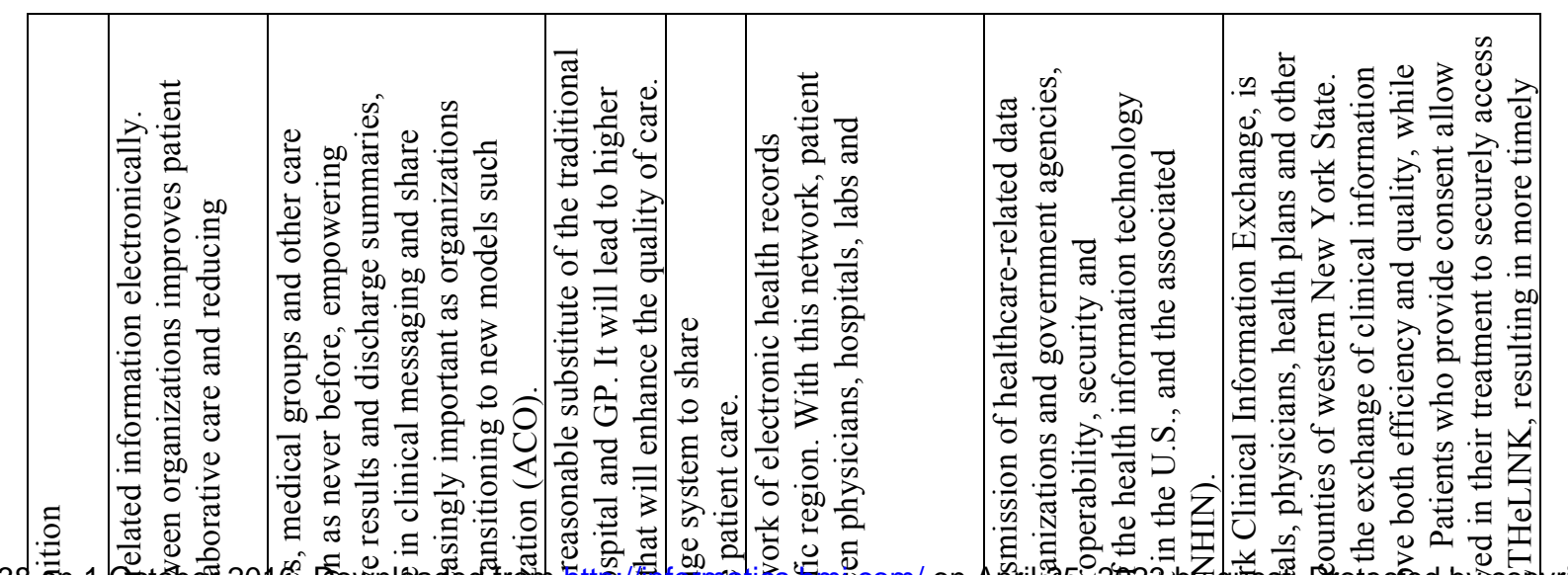

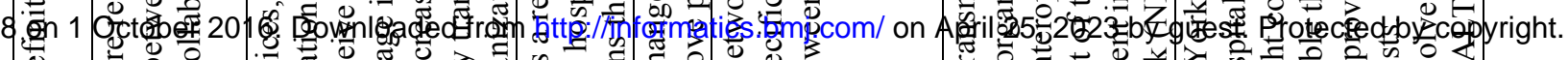

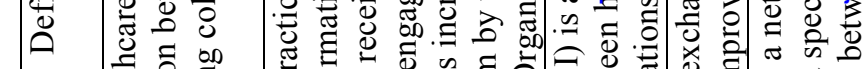

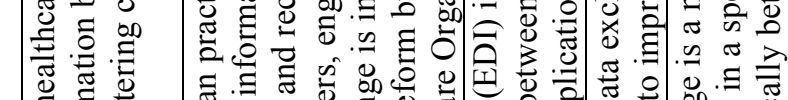

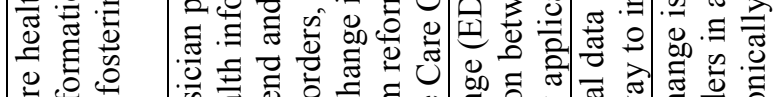

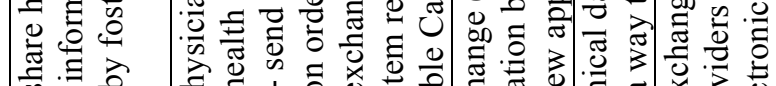
至.

\begin{tabular}{|c|c|c|c|c|c|c|c|}
\hline & 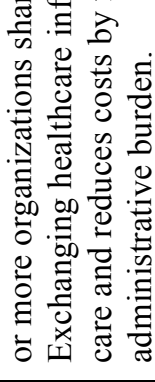 & 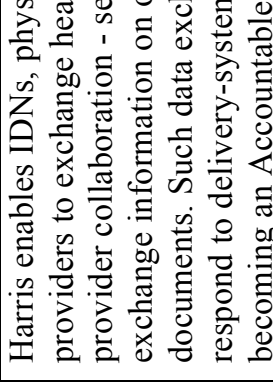 & 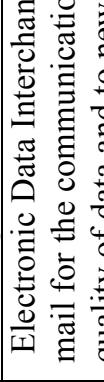 & & 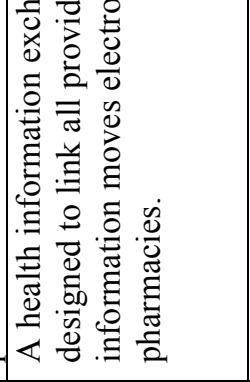 & 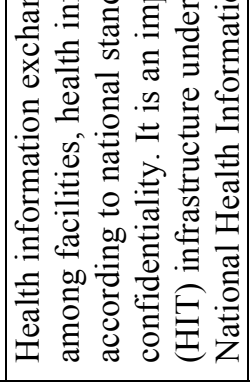 & 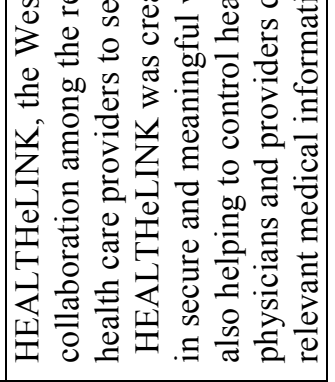 \\
\hline 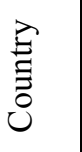 & & 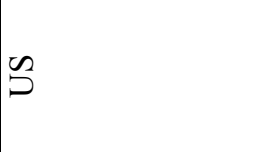 & 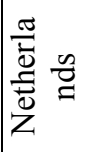 & $\mathscr{\Omega}$ & $\Omega$ & $\tilde{s}$ & $\Omega$ \\
\hline 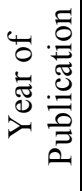 & & 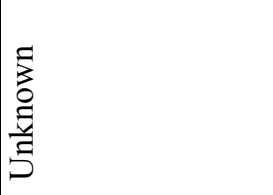 & $\tilde{\partial}$ & $\stackrel{m}{\stackrel{n}{\sim}}$ & 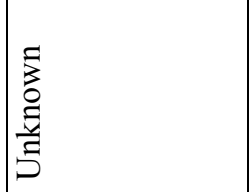 & 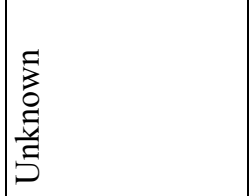 & 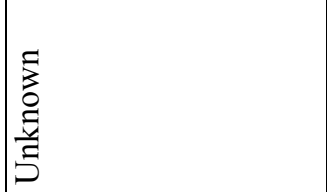 \\
\hline 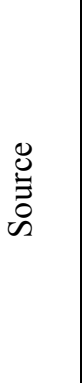 & 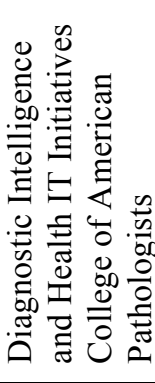 & 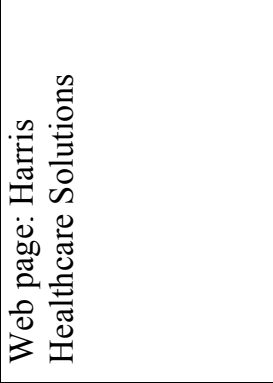 & 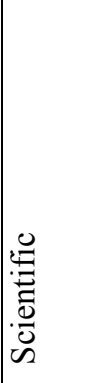 & 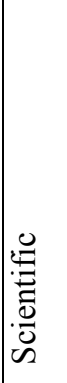 & 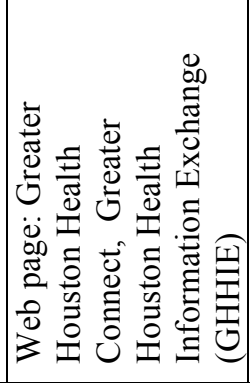 & 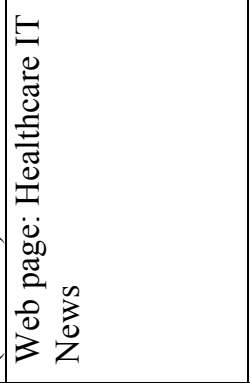 & 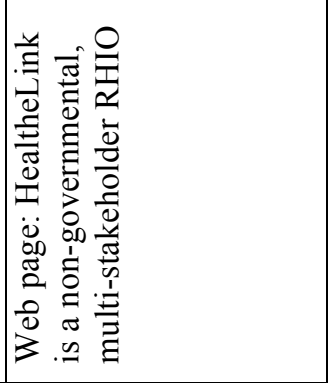 \\
\hline 蒿 & & 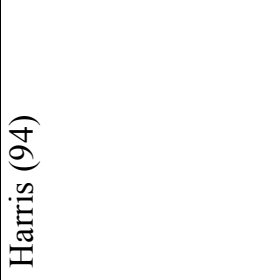 & 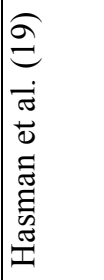 & 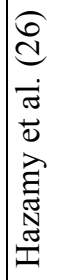 & 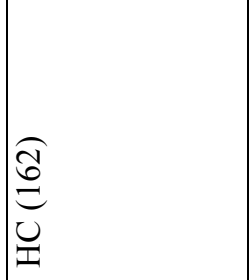 & 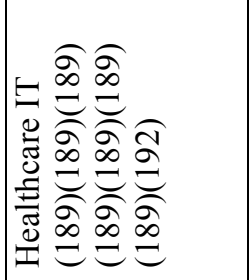 & 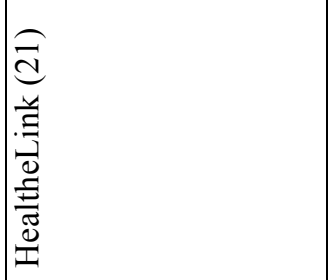 \\
\hline $\begin{array}{l}\dot{0} \\
\dot{z} \\
\dot{s}\end{array}$ & & $\infty_{\infty}$ & $\infty$ & 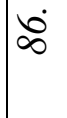 & $\hat{\infty}$ & $\infty$ & $\infty$ \\
\hline
\end{tabular}


$\stackrel{0}{N}$

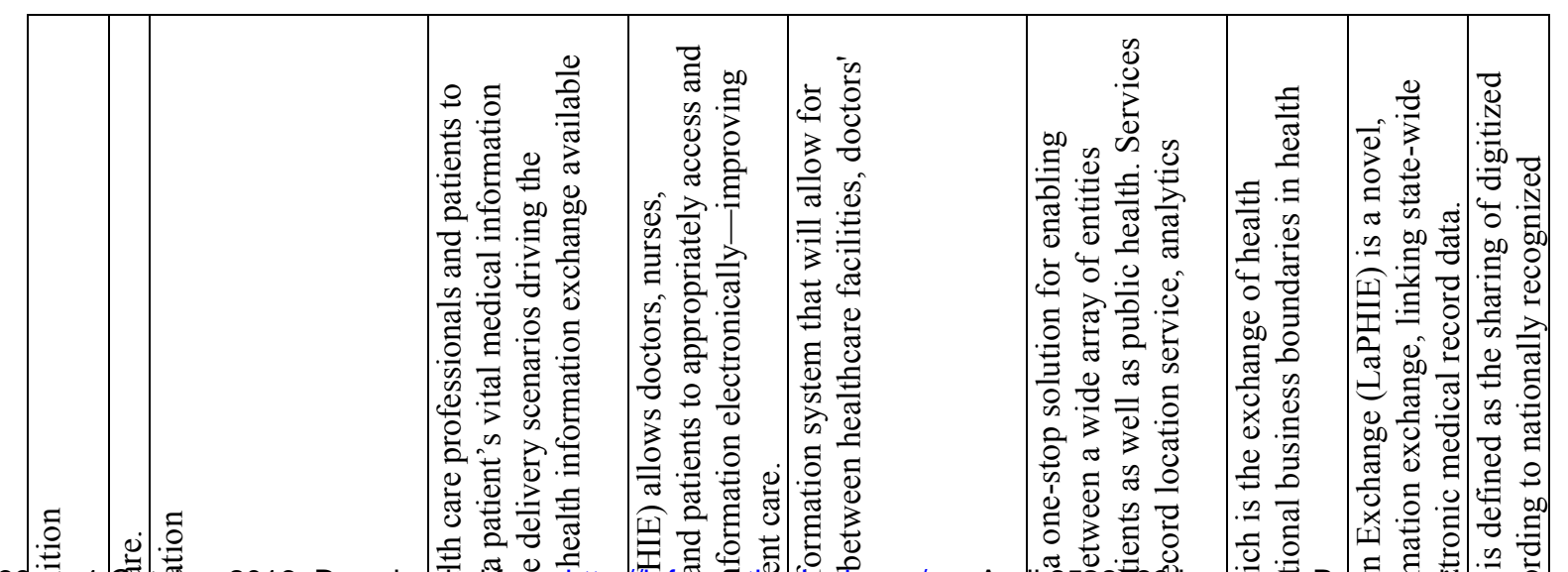

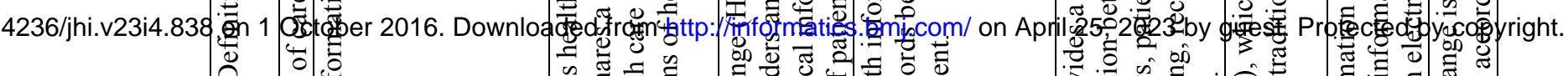

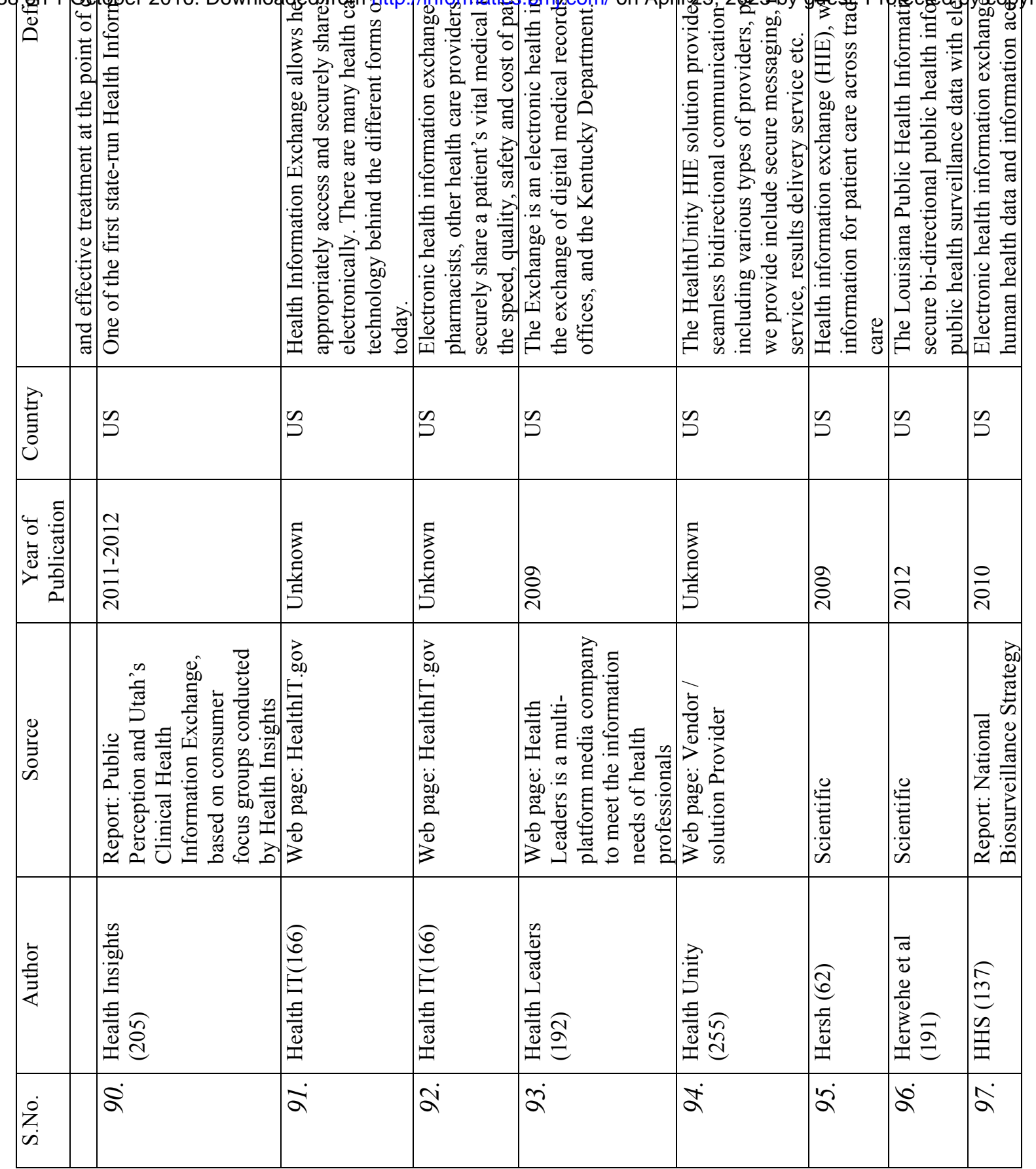


곤

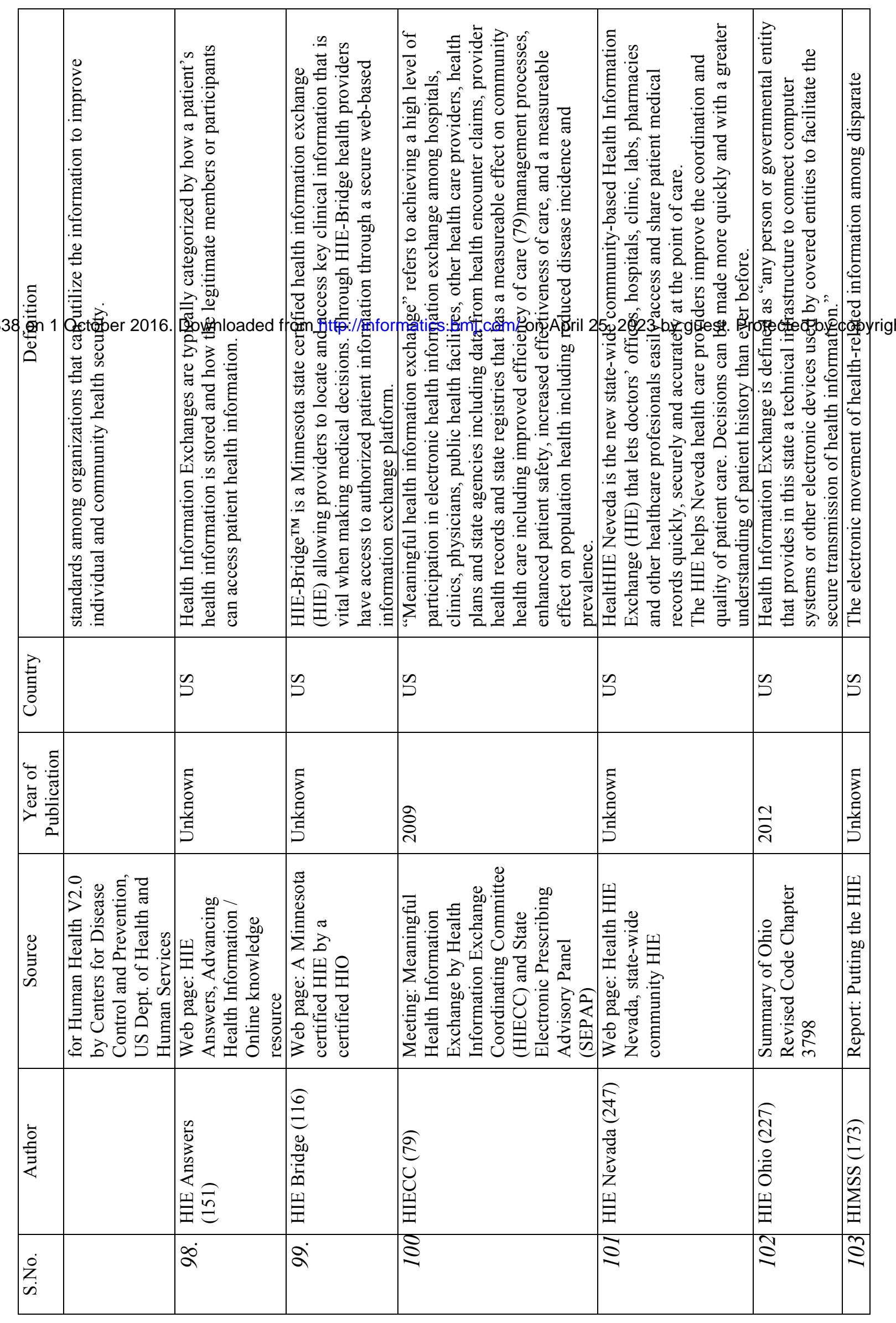


$\stackrel{\infty}{\wedge}$
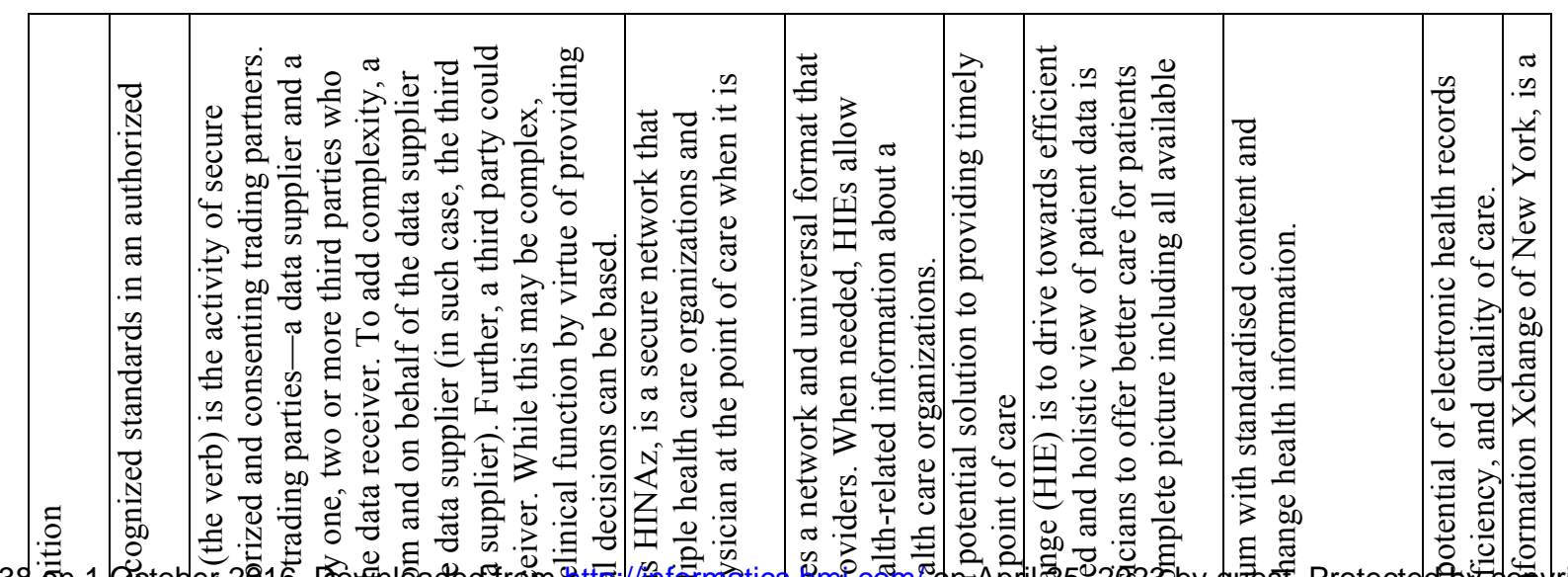

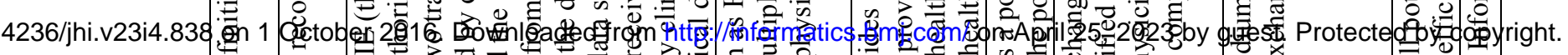

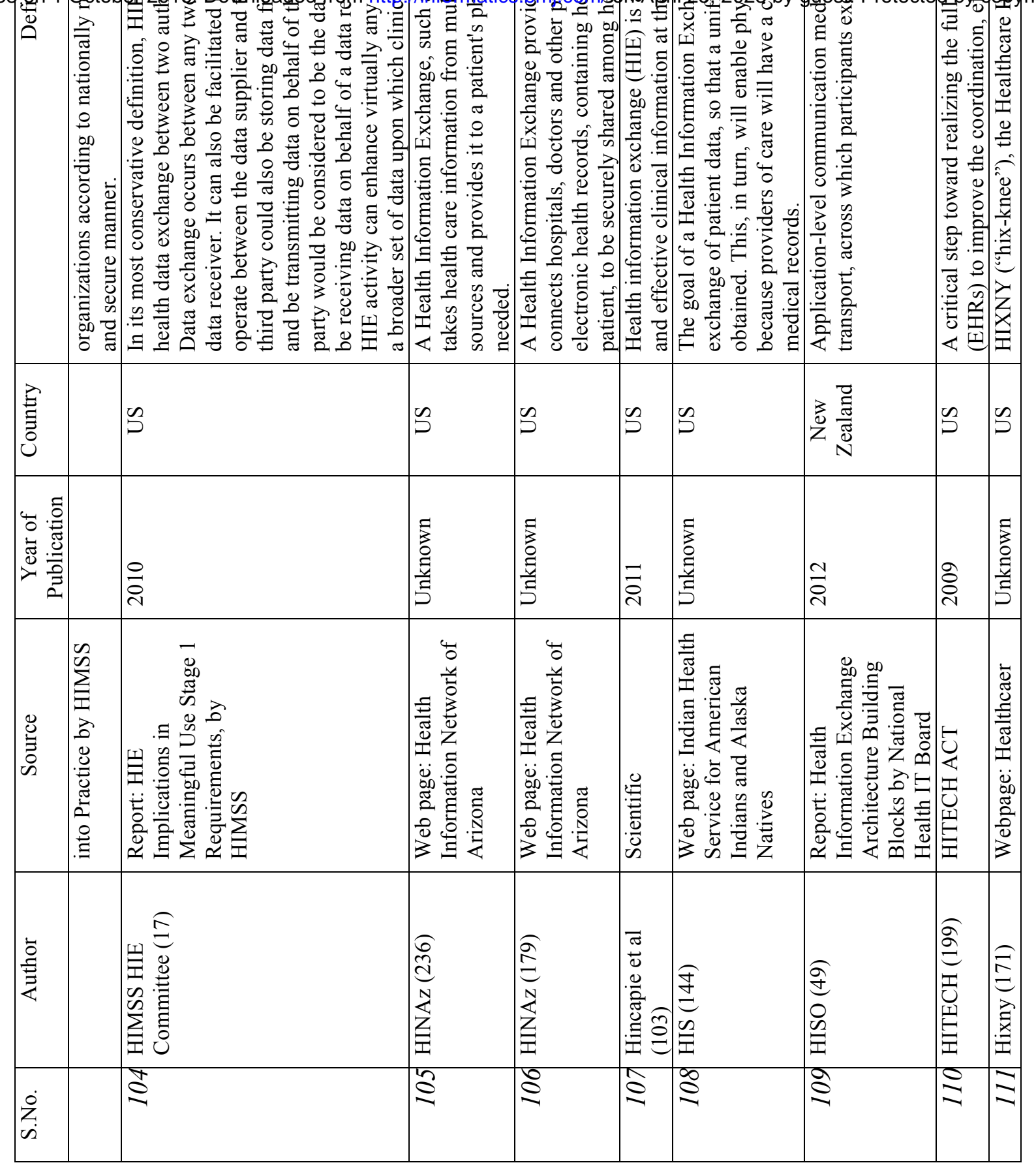


$\stackrel{9}{1}$

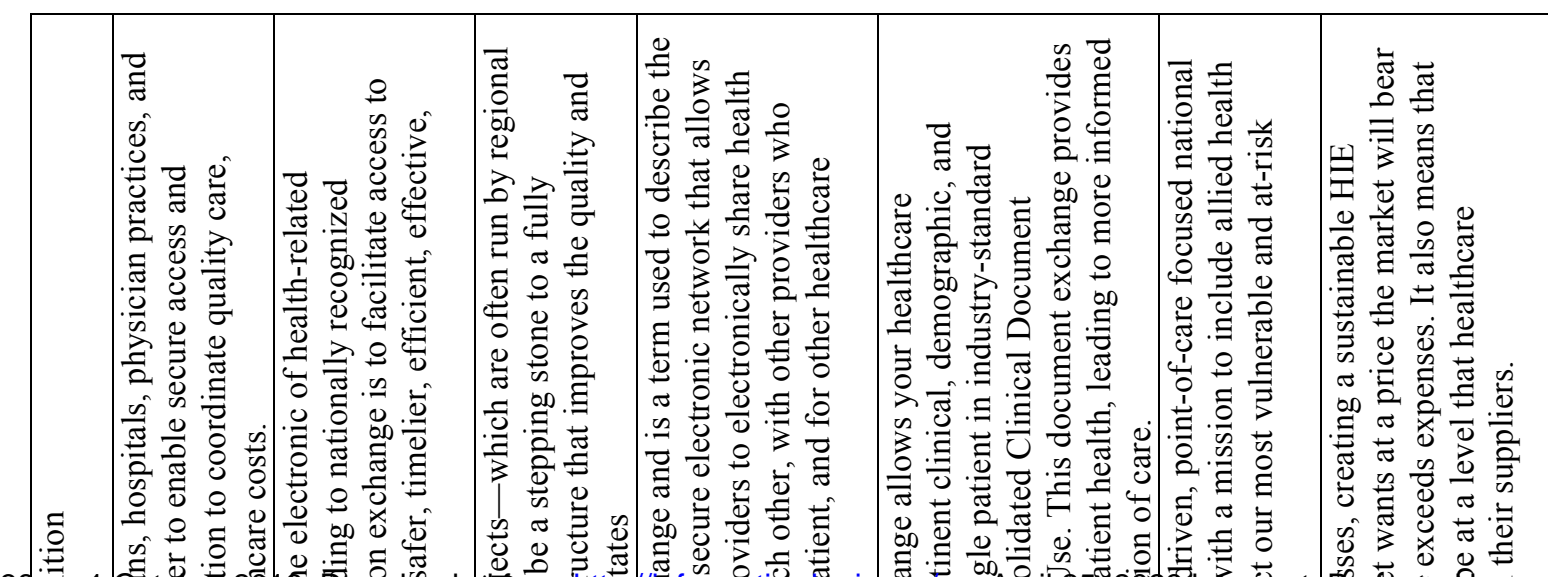

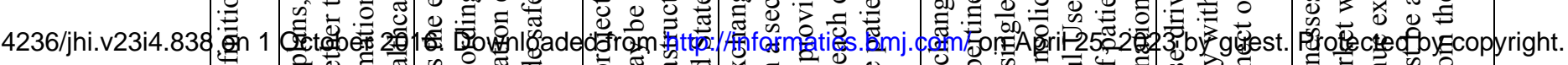

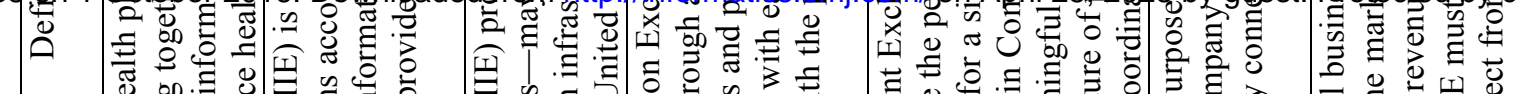

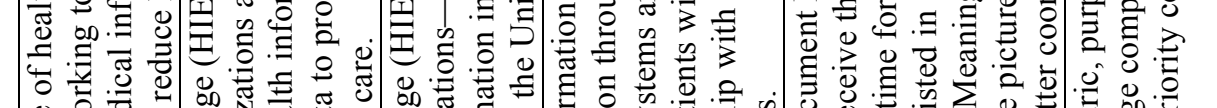

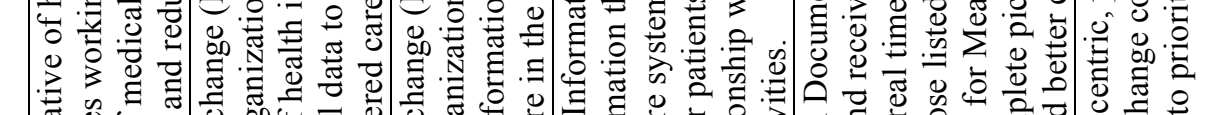

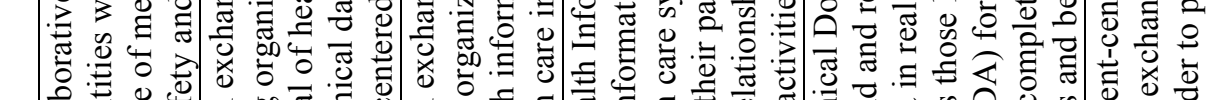

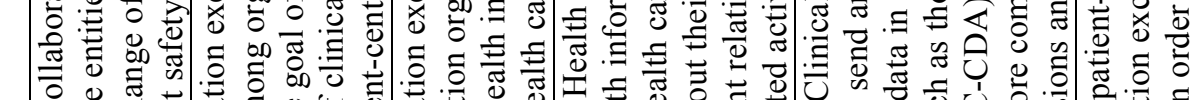

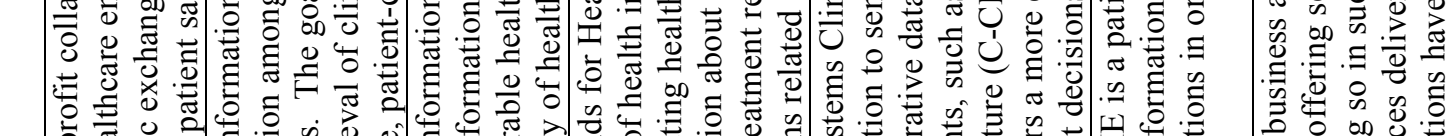

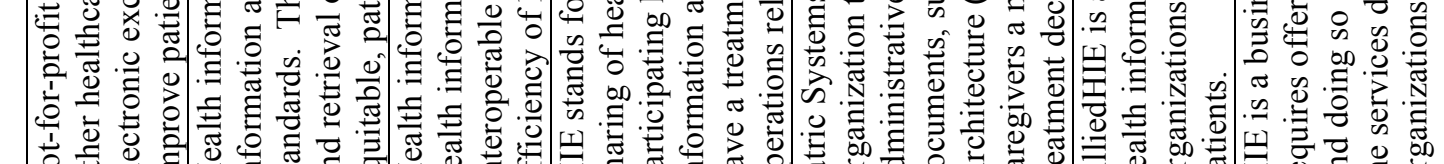

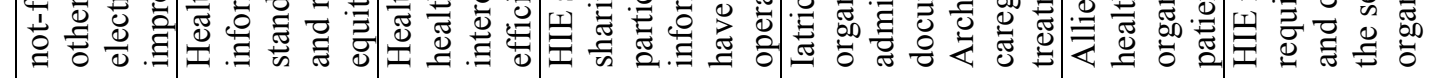

\begin{tabular}{|c|c|c|c|c|c|c|c|}
\hline $\begin{array}{l}\stackrel{\vec{E}}{\Xi} \\
\stackrel{0}{0}\end{array}$ & & $\tilde{\rho}$ & $\tilde{s}$ & $\mathscr{2}$ & $\tilde{s}$ & 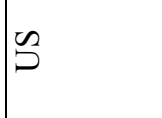 & 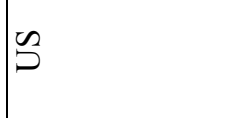 \\
\hline 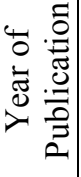 & & 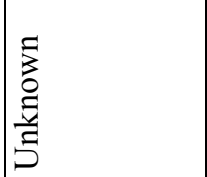 & 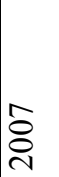 & 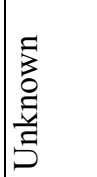 & 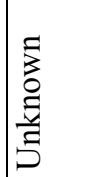 & $\frac{\sim}{\stackrel{\sim}{*}}$ & 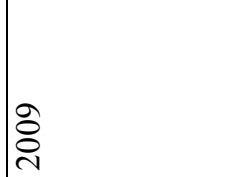 \\
\hline 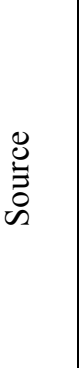 & 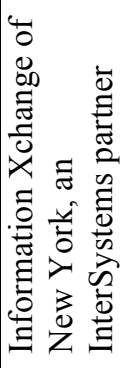 & 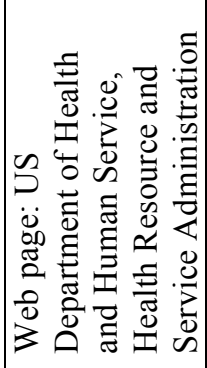 & 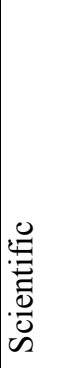 & 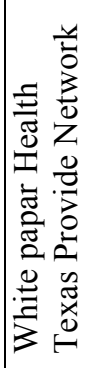 & 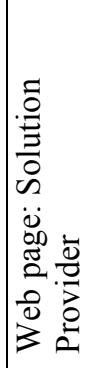 & 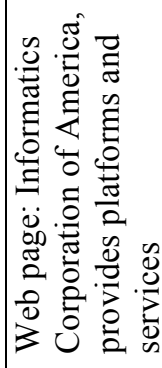 & 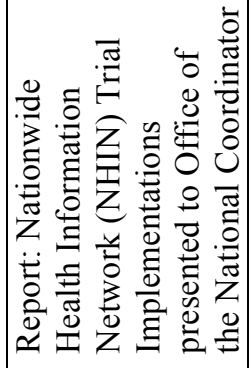 \\
\hline 竧 & & 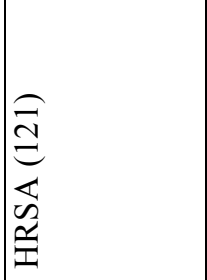 & 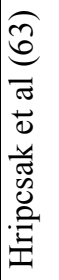 & $\begin{array}{l}\mathscr{0} \\
\infty \\
\Xi \\
\Xi\end{array}$ & $\widehat{\overparen{d}}$ & 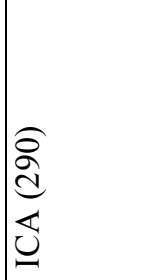 & 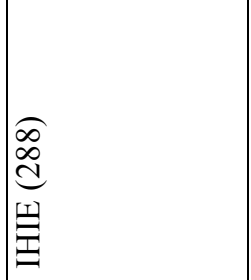 \\
\hline 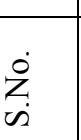 & & $\triangleq$ & 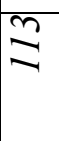 & \pm & 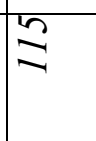 & 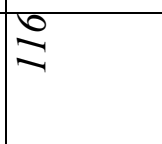 & $\lesssim$ \\
\hline
\end{tabular}


곡

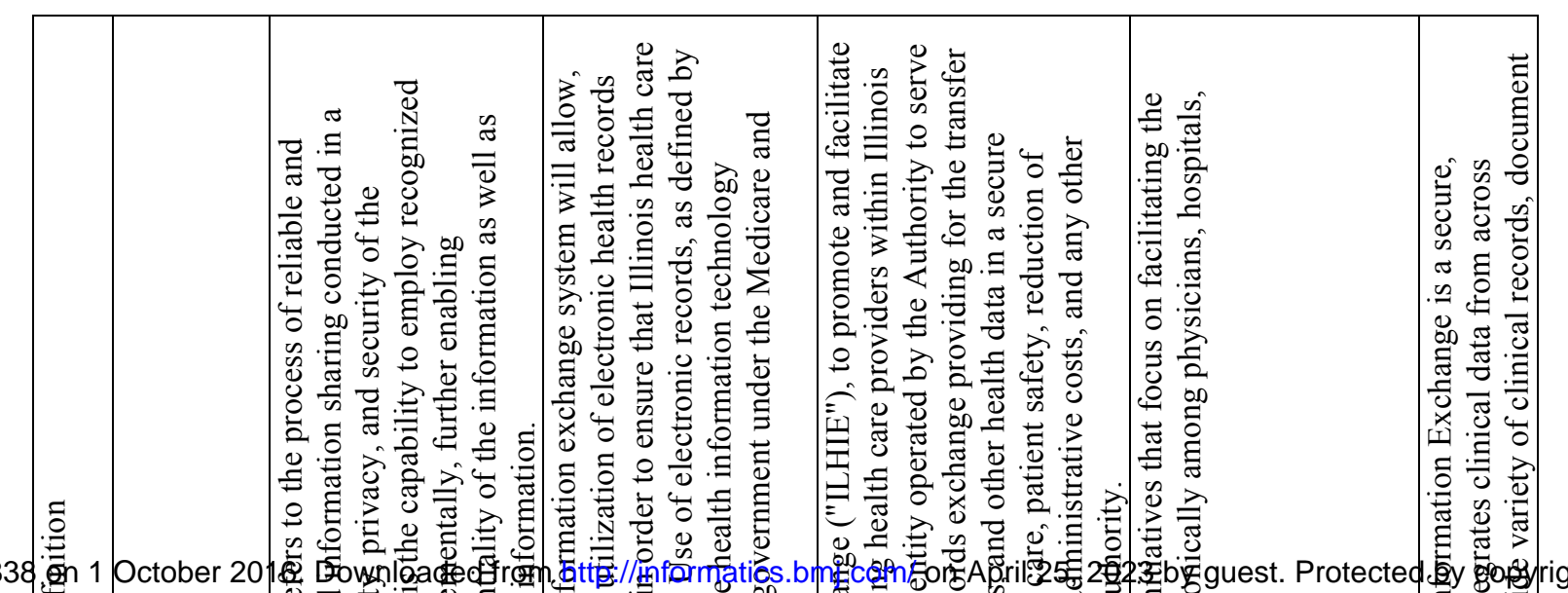

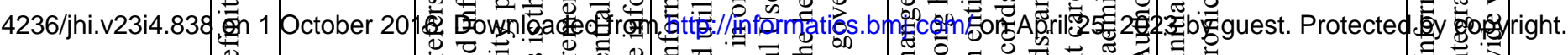

\begin{tabular}{|c|c|c|c|c|c|c|}
\hline & & 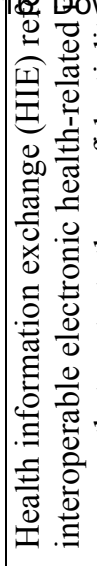 & 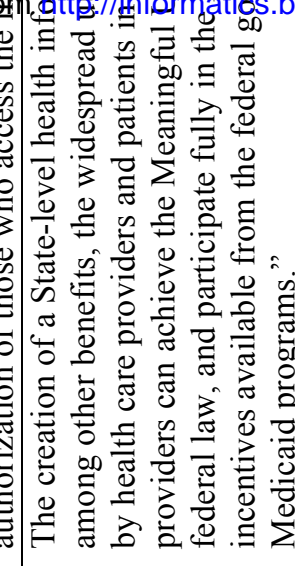 & 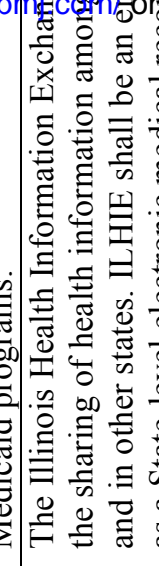 & 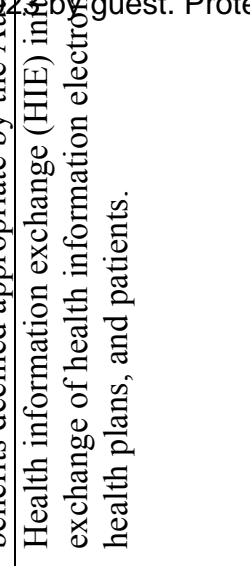 & 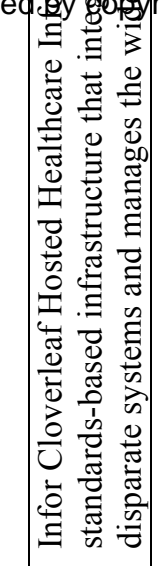 \\
\hline 局 & & $\begin{array}{l}\frac{z}{0} \\
\frac{\underline{y}}{5}\end{array}=$ & s & $\tilde{s}$ & $n$ & 5 \\
\hline 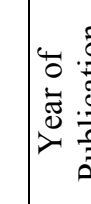 & & 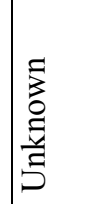 & 高 & 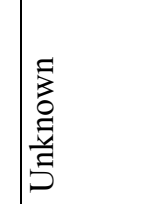 & 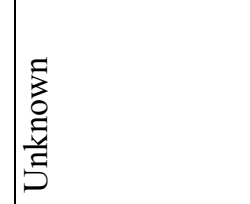 & 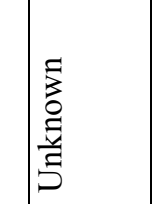 \\
\hline 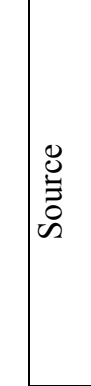 & 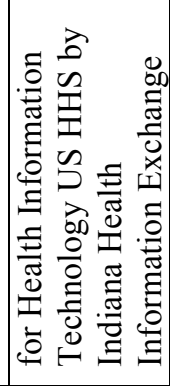 & 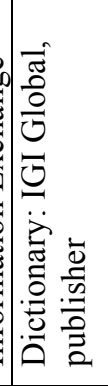 & 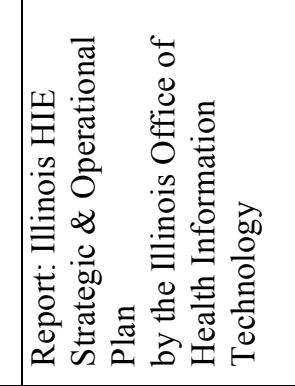 & 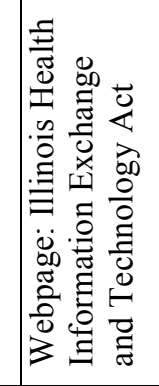 & 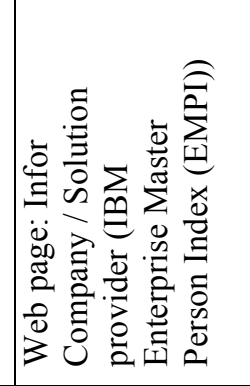 & 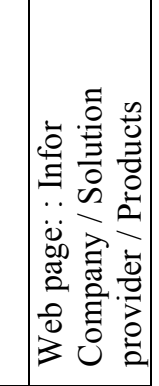 \\
\hline 总 & & 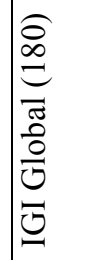 & 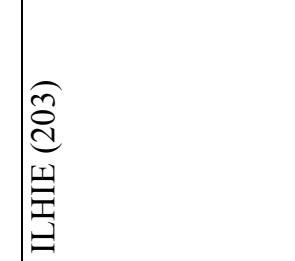 & 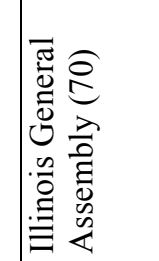 & 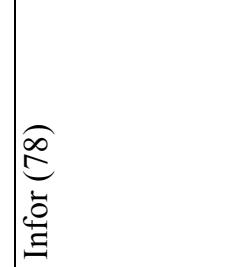 & 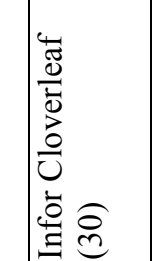 \\
\hline $\begin{array}{l}\dot{s} \\
\dot{z} \\
\dot{c}\end{array}$ & & $\stackrel{\infty}{=}$ & $\stackrel{\nexists}{\beth}$ & 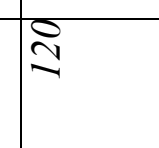 & $\bar{\Xi}$ & 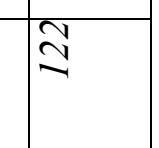 \\
\hline
\end{tabular}




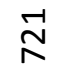

\begin{tabular}{|c|c|c|c|c|c|c|c|}
\hline $\mid$ & 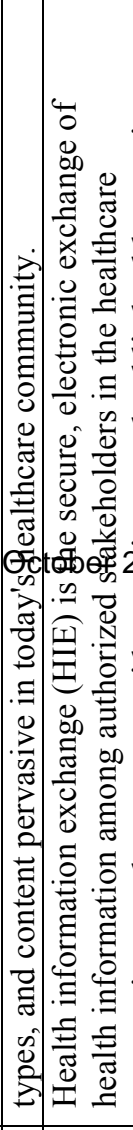 & 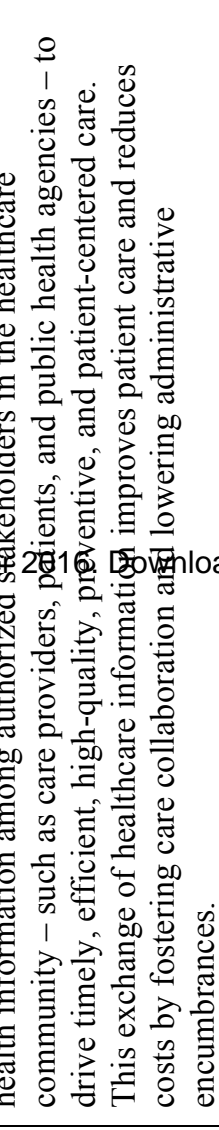 & 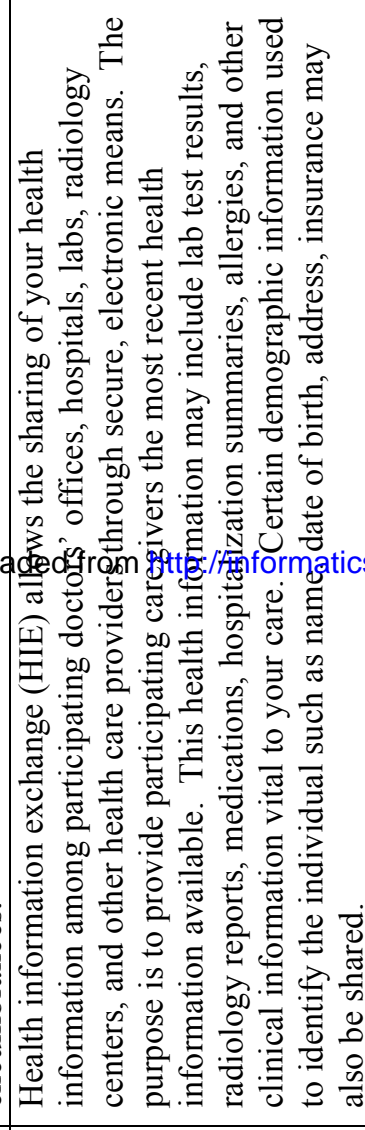 & 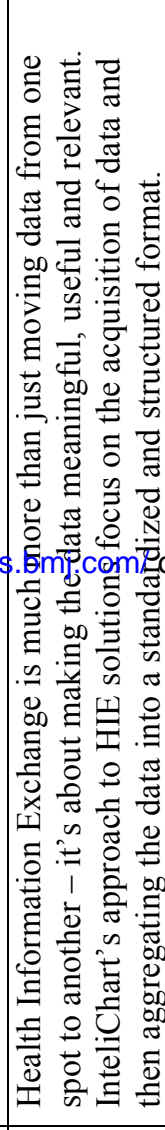 & 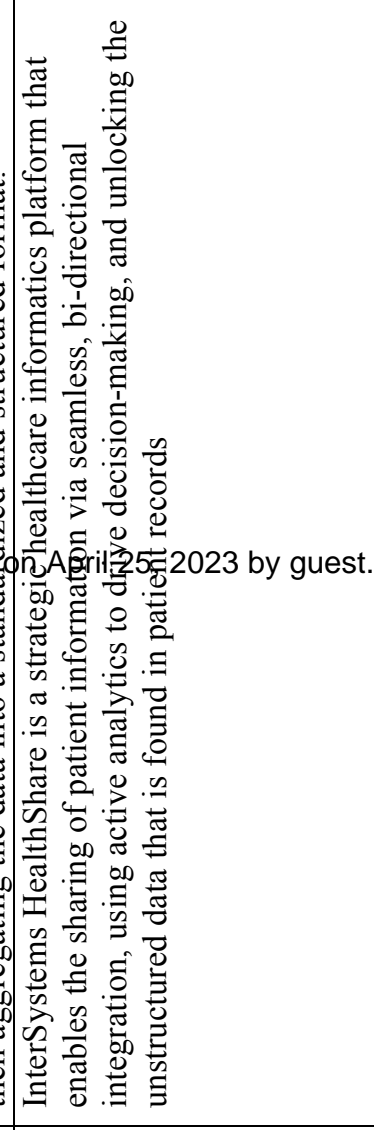 & 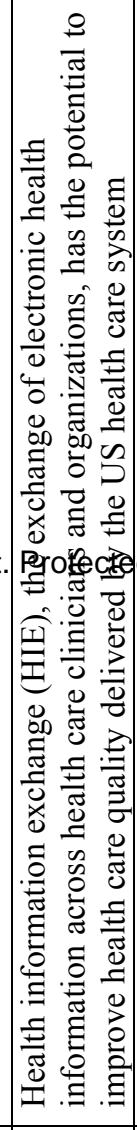 & 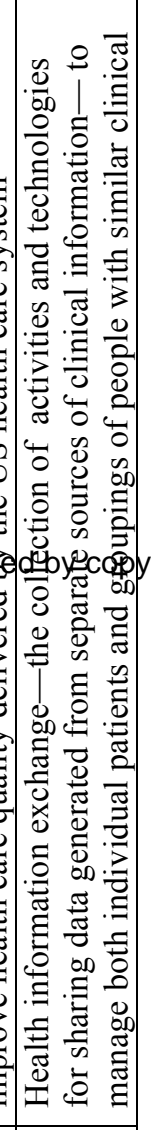 \\
\hline 童 & 12 & & 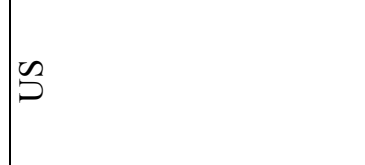 & $\tilde{s}$ & 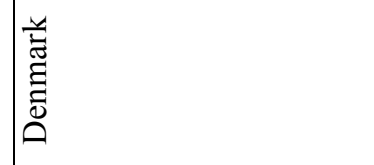 & $\tilde{\rho}$ & $\tilde{\Omega}$ \\
\hline 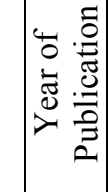 & 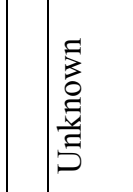 & & 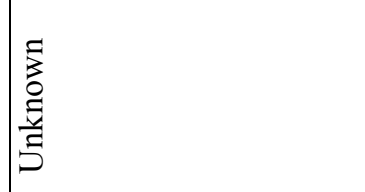 & 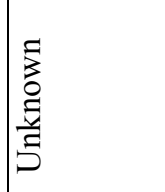 & స̃ & ¿্ণ & $\overrightarrow{\vec{\sim}}$ \\
\hline 䓂 & 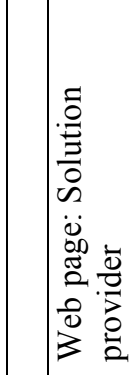 & & 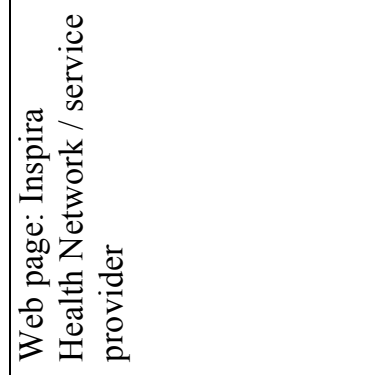 & 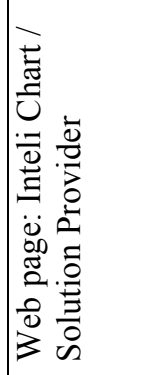 & 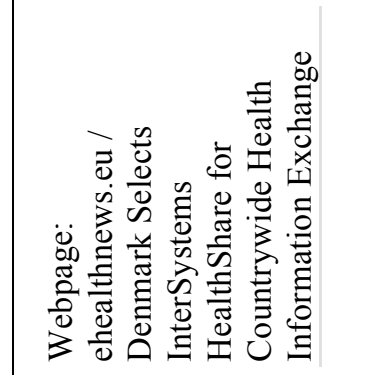 & 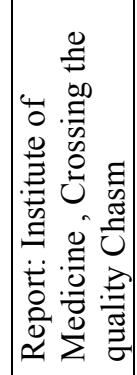 & \\
\hline 芩 & 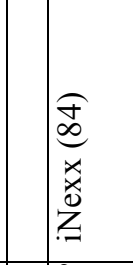 & & 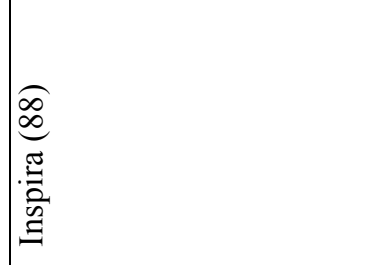 & 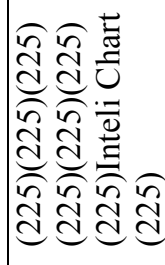 & 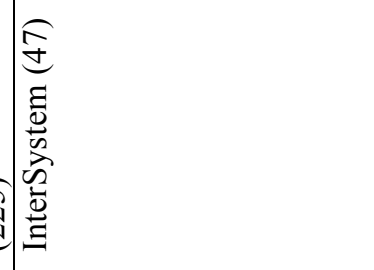 & 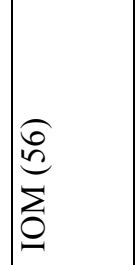 & $\underset{\sim}{\stackrel{d}{d}}$ \\
\hline$\ddot{z}$ & $\stackrel{\cong}{\beth}$ & & $\stackrel{ \pm}{\beth}$ & $\cong$ & $\stackrel{\ominus}{\cong}$ & $\approx$ & $\stackrel{\infty}{\beth}$ \\
\hline
\end{tabular}


กี

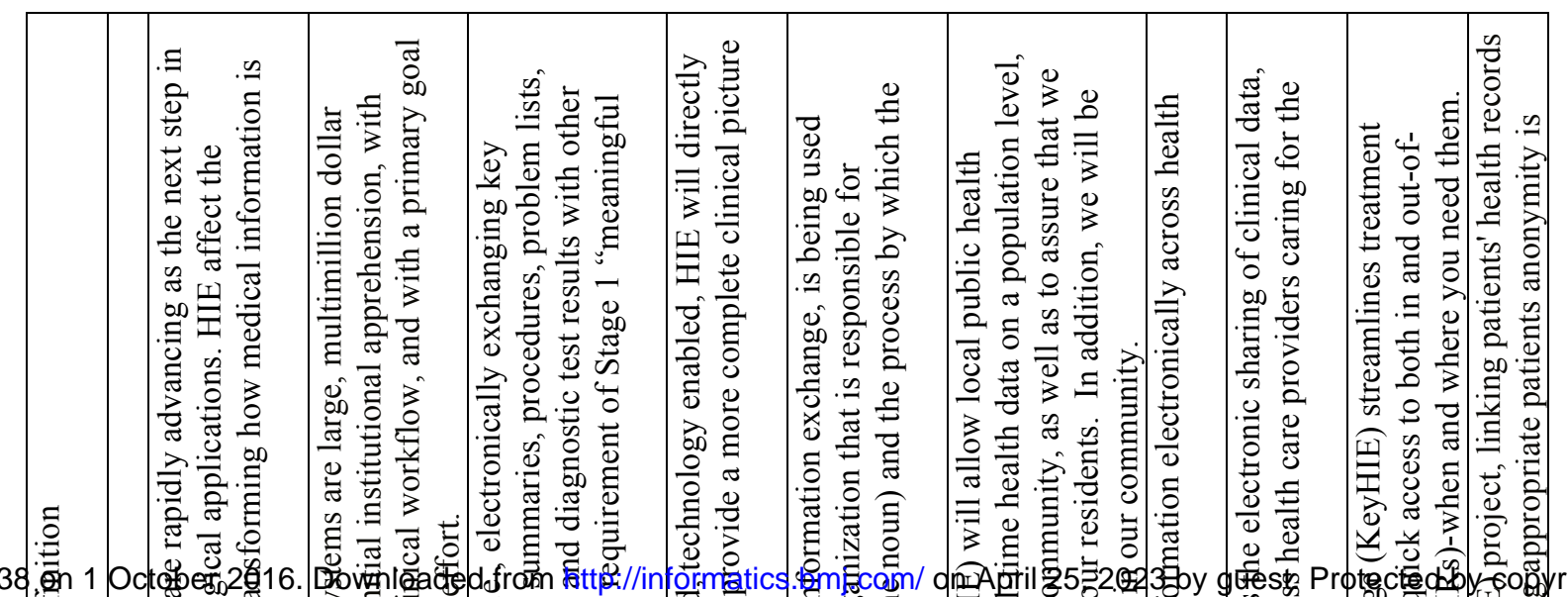

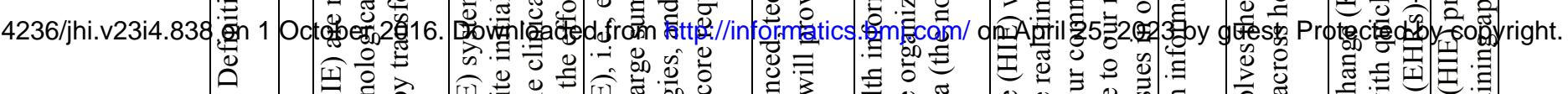

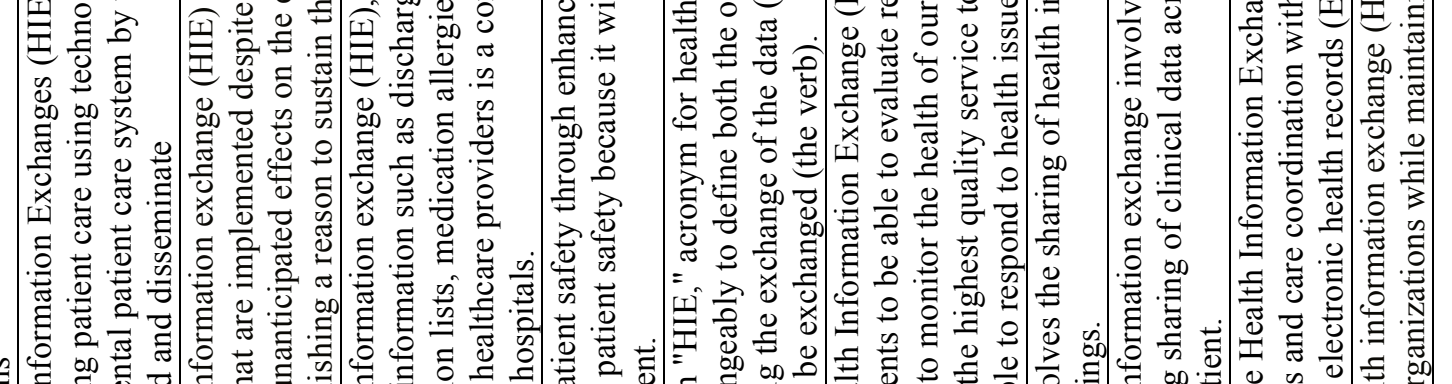

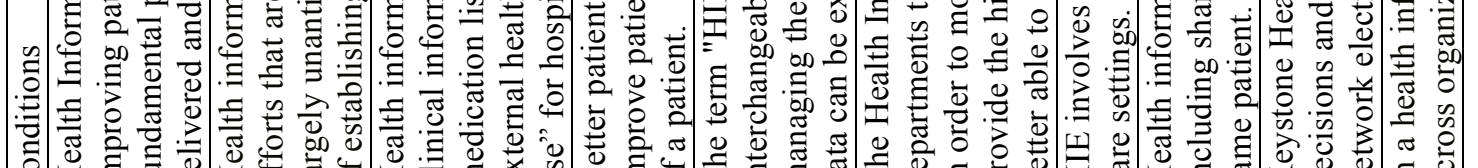

\begin{tabular}{|c|c|c|c|c|c|c|c|c|c|c|}
\hline 壭 & 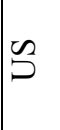 & $\tilde{\Omega}$ & $\tilde{\rho}$ & 2 & 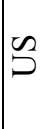 & $\tilde{s}$ & $\tilde{s}$ & $\Omega$ & $\Omega$ & $\tilde{\Omega}$ \\
\hline 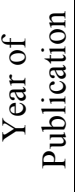 & $\stackrel{\circ}{\stackrel{0}{\sim}}$ & 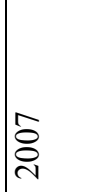 & $\overrightarrow{\vec{d}}$ & $\widehat{\stackrel{े}{े}}$ & $\stackrel{\sim}{\stackrel{\sim}{\sim}}$ & 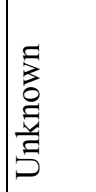 & ڤ్̀ & 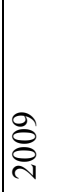 & 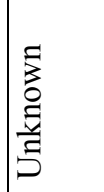 & ڤ్̀ \\
\hline 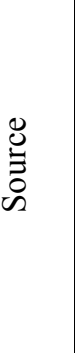 & 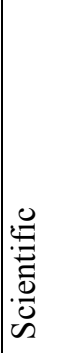 & 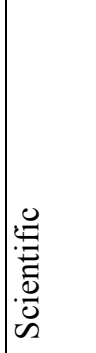 & 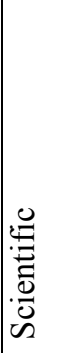 & 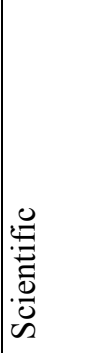 & 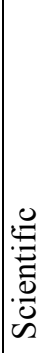 & 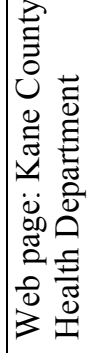 & 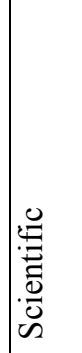 & 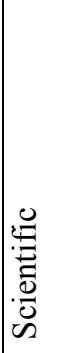 & 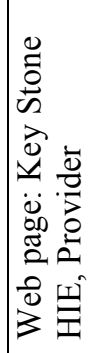 & 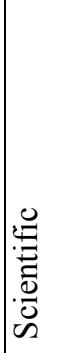 \\
\hline 宔 & 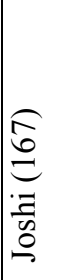 & 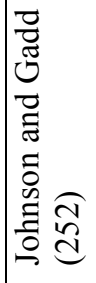 & $\begin{array}{l}\widehat{\sigma} \\
0 \\
\Xi \\
\pi \\
0 \\
0 \\
0 \\
0 \\
0 \\
0\end{array}$ & 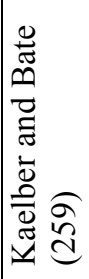 & 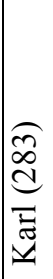 & 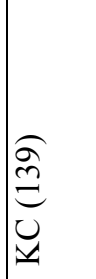 & 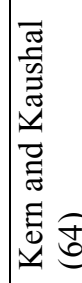 & 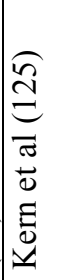 & 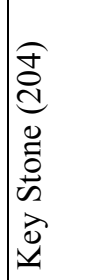 & 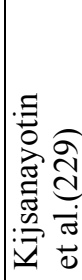 \\
\hline $\begin{array}{l}\dot{z} \\
\dot{z} \\
\text { is }\end{array}$ & $\stackrel{\text { I }}{\beth}$ & $\stackrel{m}{\sim}$ & $\bar{m}$ & $\stackrel{\approx}{\sim}$ & $\ddot{\sim}$ & $\stackrel{m}{2}$ & $\begin{array}{l}\stackrel{\sim}{n} \\
=\end{array}$ & 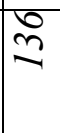 & $\hat{m}$ & $\stackrel{\infty}{m}$ \\
\hline
\end{tabular}


$\stackrel{n}{\Sigma}$
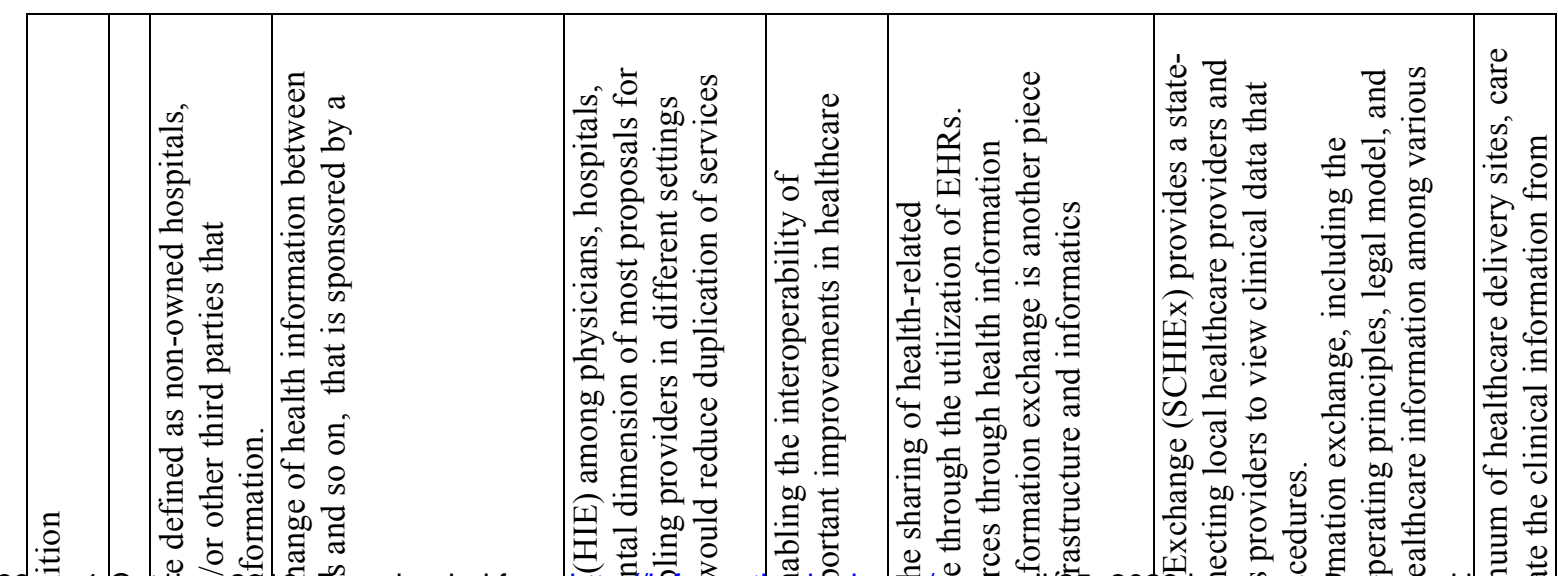

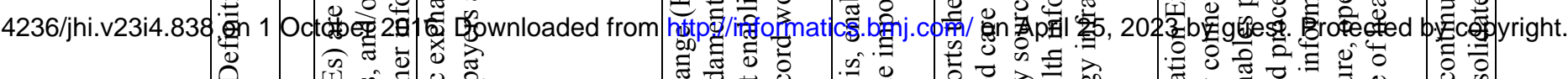

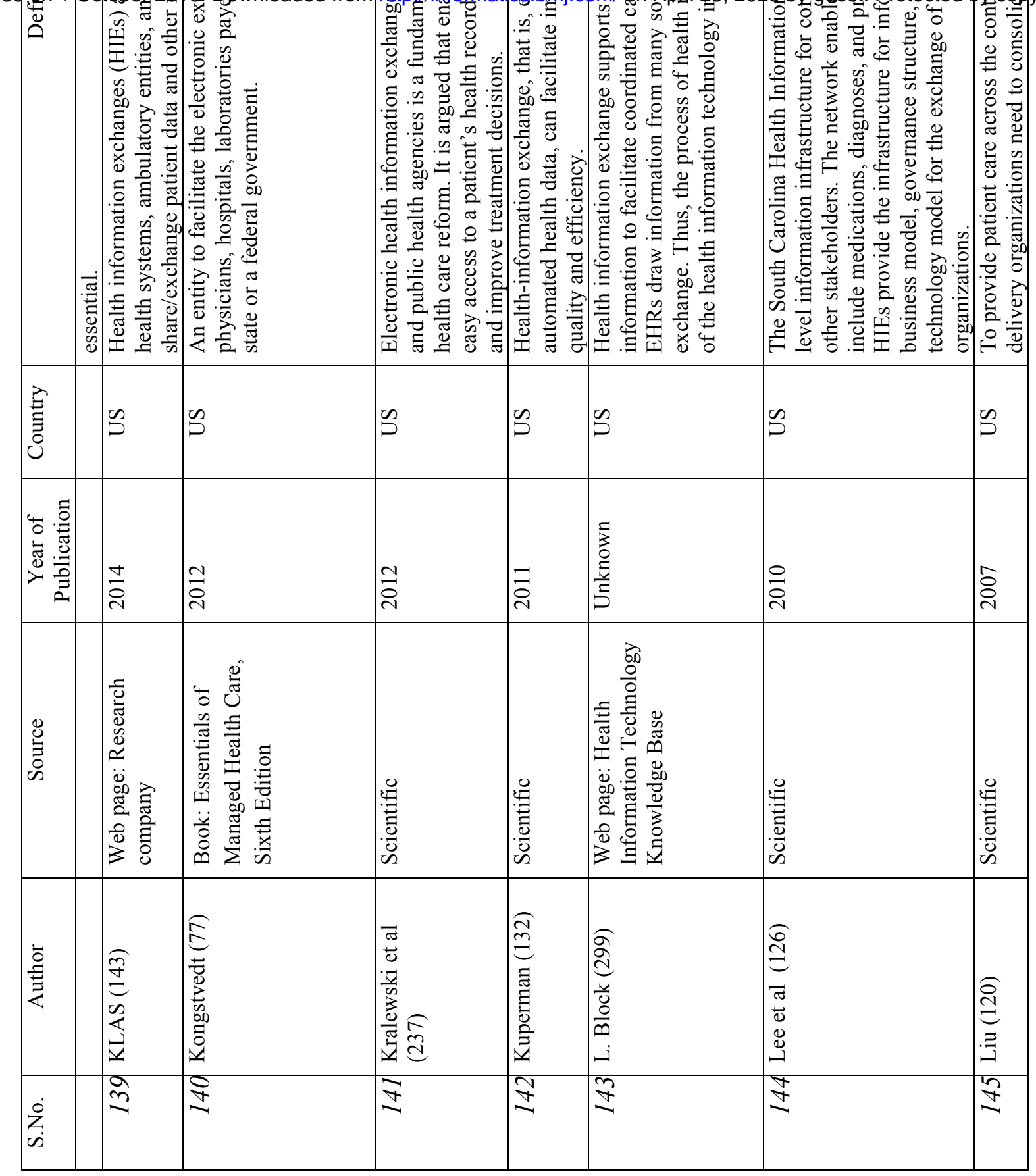


$\stackrel{N}{N}$

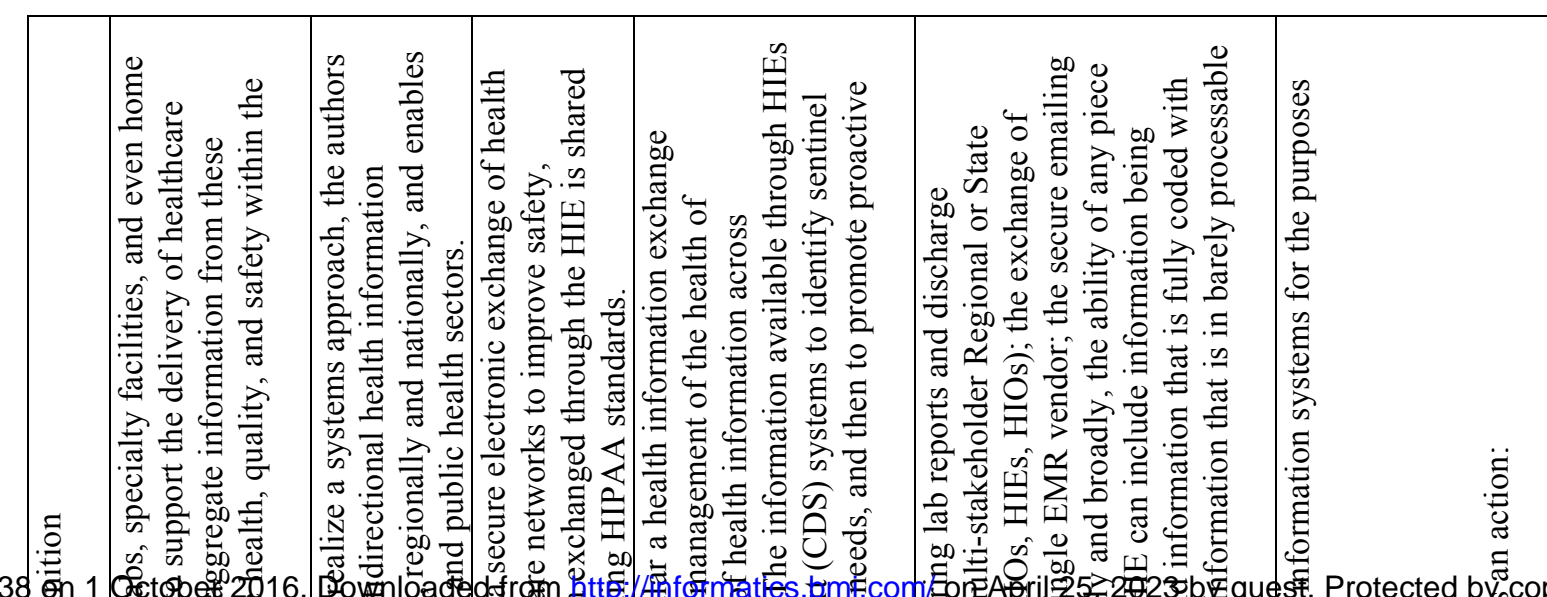

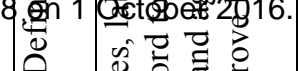

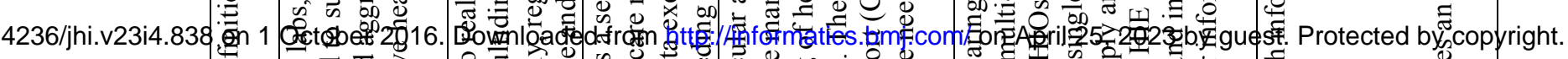

\begin{tabular}{|c|c|c|c|c|c|c|}
\hline صّ & 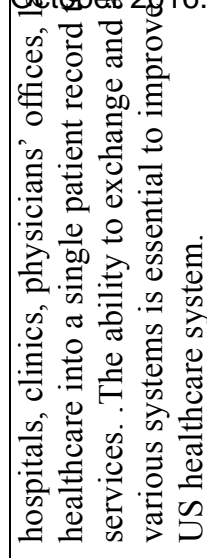 & 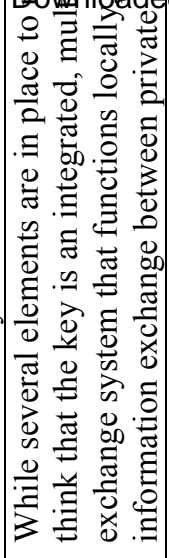 & 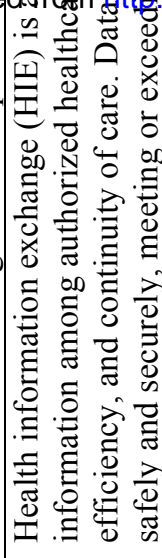 & 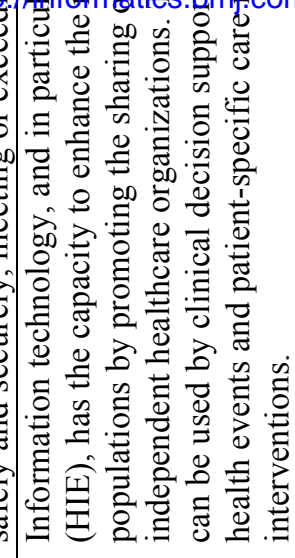 & 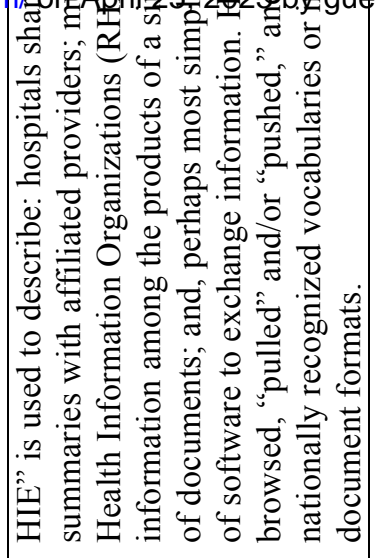 & 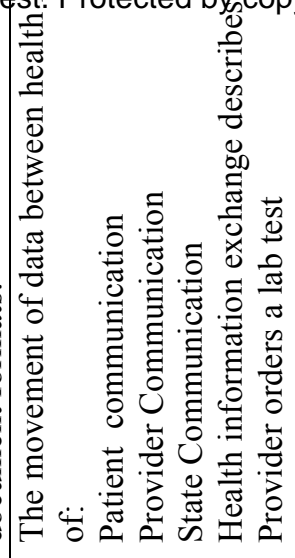 \\
\hline $\begin{array}{l}\hat{E} \\
\text { 音 } \\
0\end{array}$ & & $\tilde{s}$ & $\tilde{s}$ & 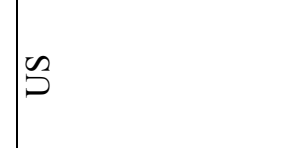 & $\tilde{s}$ & $\Omega$ \\
\hline 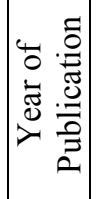 & & $\stackrel{\circ}{\stackrel{i}{\circ}}$ & 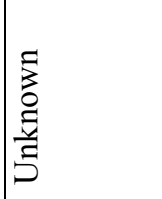 & ¿े & $\stackrel{0}{\stackrel{0}{\circ}}$ & 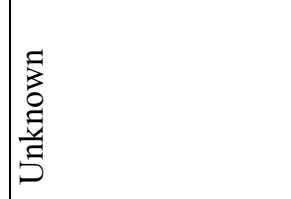 \\
\hline 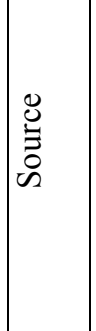 & & 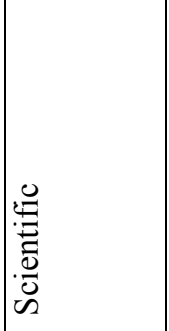 & 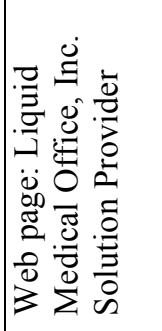 & 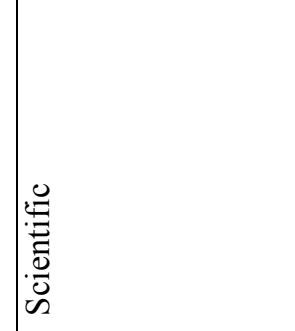 & 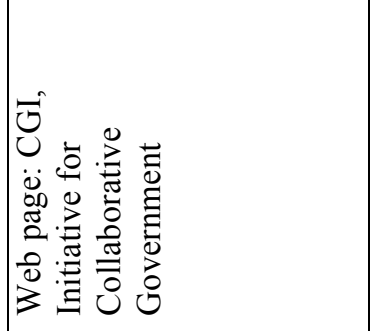 & 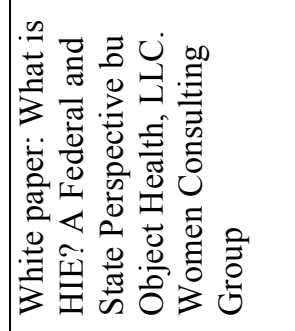 \\
\hline 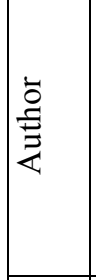 & & 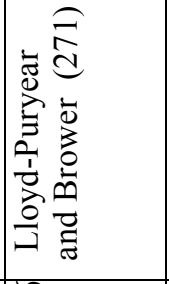 & 同 & $\begin{array}{l}\sqrt{n} \\
0 \\
\frac{\pi}{\pi} \\
0 \\
0 \\
0 \\
\tilde{0} \\
0 \\
0 \\
1\end{array}$ & 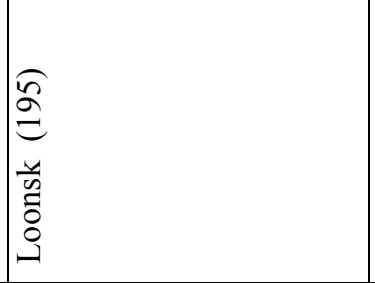 & 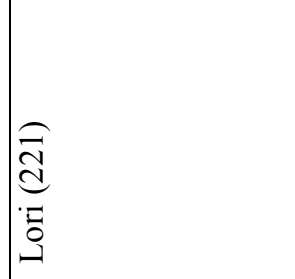 \\
\hline $\begin{array}{l}0 \\
z \\
\dot{n} \\
\dot{n}\end{array}$ & & $\stackrel{\circ}{\sim}$ & $\underset{\nabla}{Z}$ & $\underset{\sim}{ \pm}$ & $\stackrel{\partial}{7}$ & $\stackrel{n}{=}$ \\
\hline
\end{tabular}


$\stackrel{\sim}{N}$

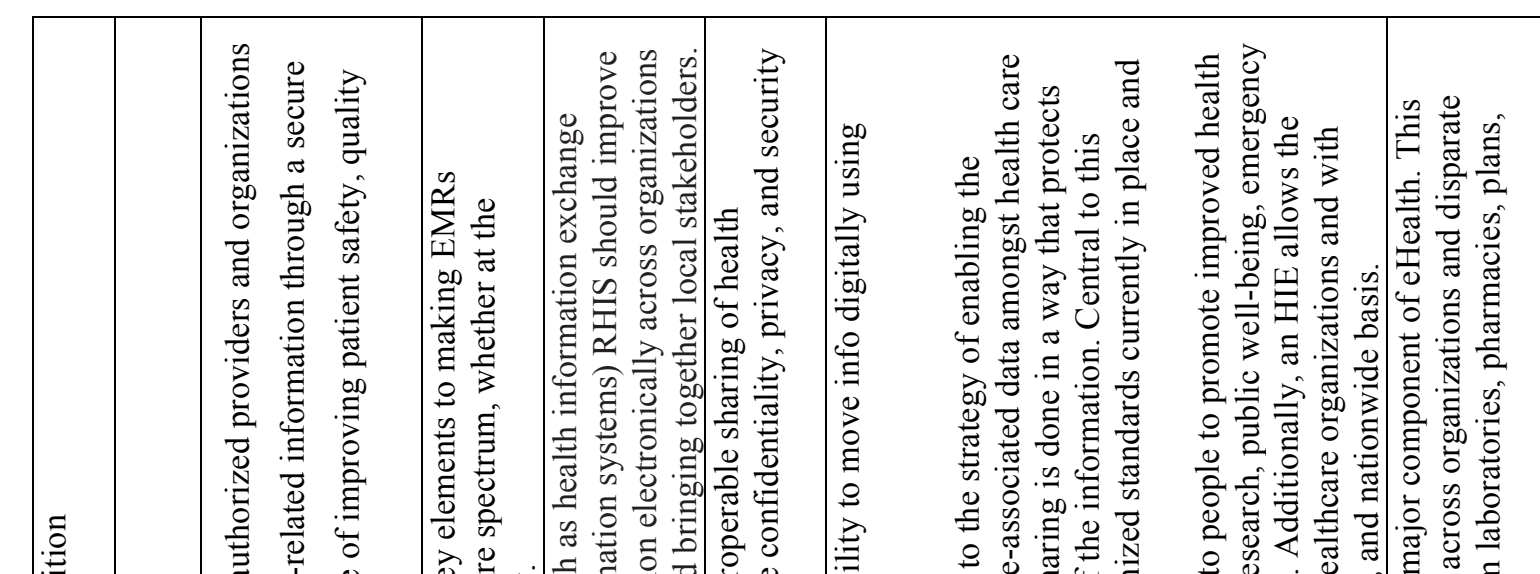

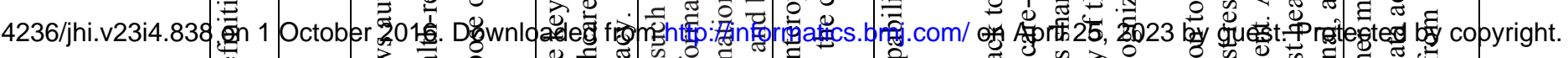

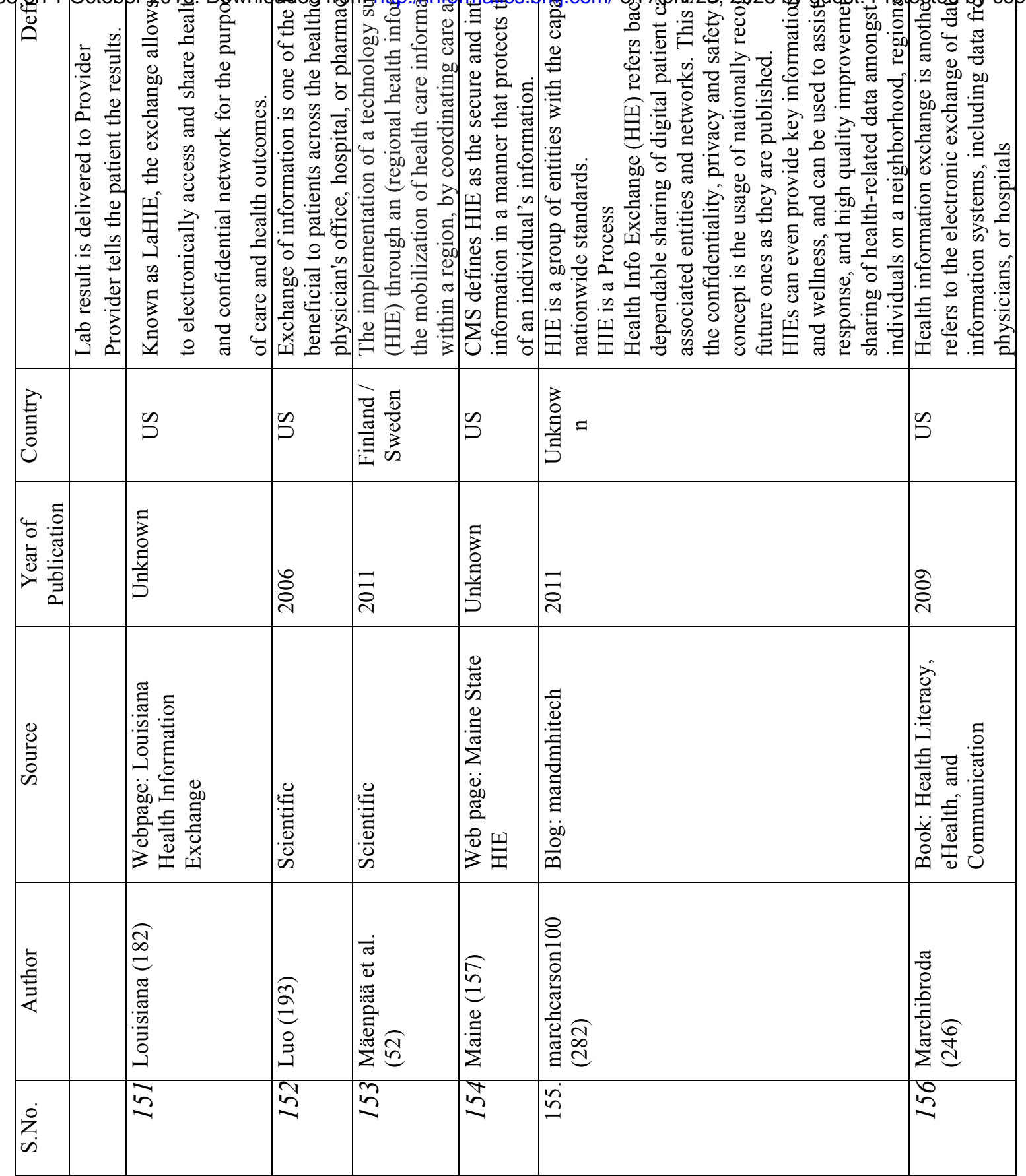


$\stackrel{\mathscr{N}}{N}$

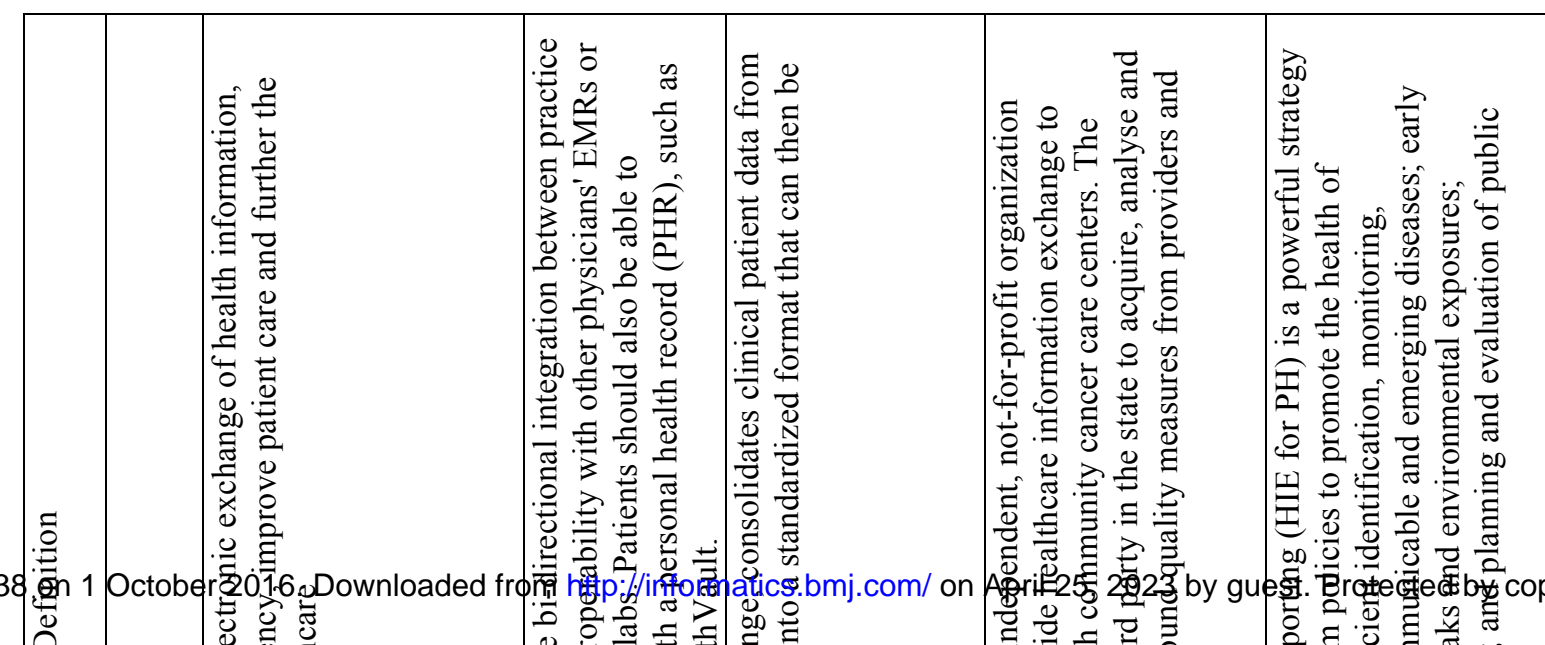

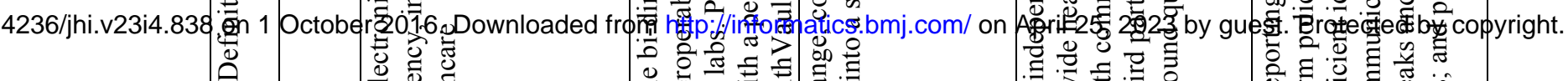

\begin{tabular}{|c|c|c|c|c|c|}
\hline صٌ & 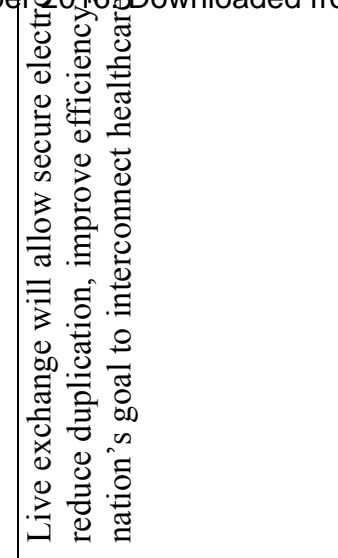 & 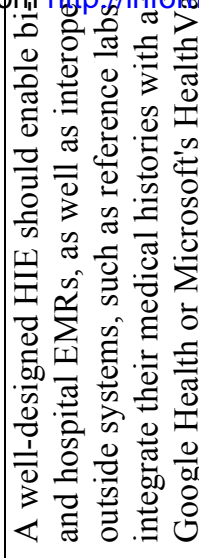 & 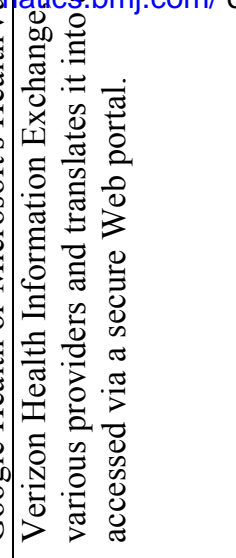 & 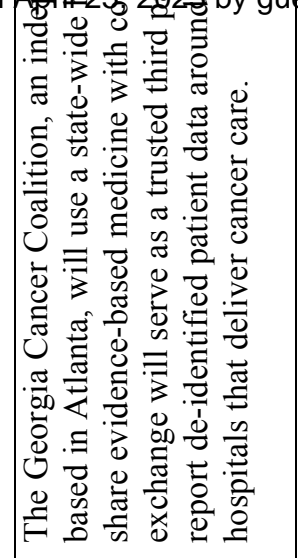 & 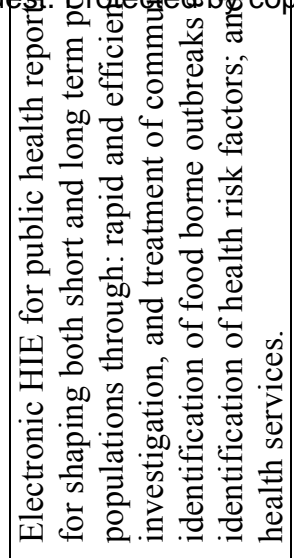 \\
\hline $\begin{array}{l}\overrightarrow{\mathrm{E}} \\
\mathrm{E} \\
\mathrm{O}\end{array}$ & $\mathscr{\Omega}$ & $\Omega$ & $\Omega$ & $\mathscr{\Omega}$ & $\Omega$ \\
\hline 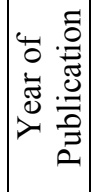 & ڤें & ஓे & $\stackrel{\circ}{\stackrel{2}{c}}$ & ڤे & $\frac{m}{\stackrel{\sim}{c}}$ \\
\hline 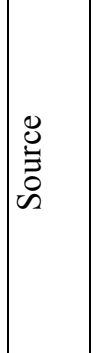 & 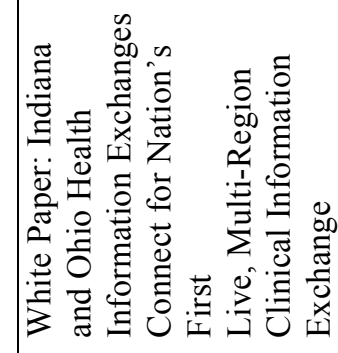 & . & 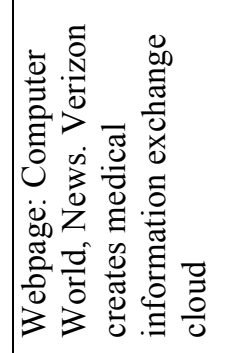 & 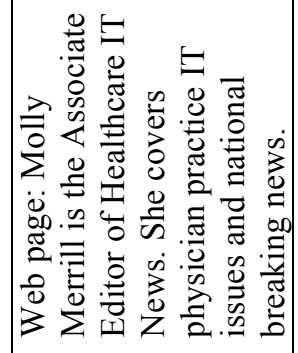 & 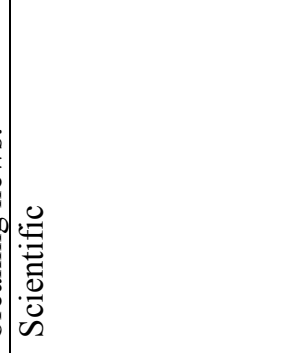 \\
\hline 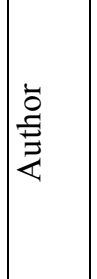 & 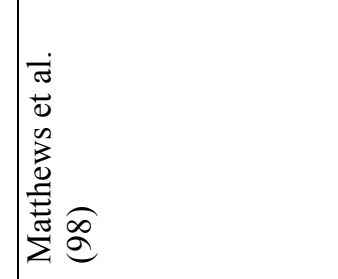 & 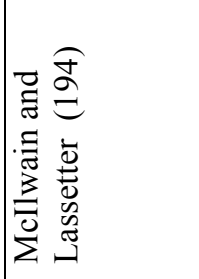 & $\stackrel{\widehat{\vartheta}}{\Xi}$ & 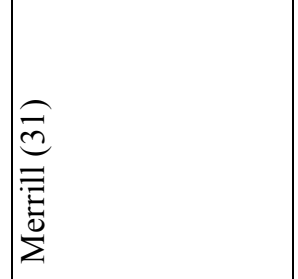 & 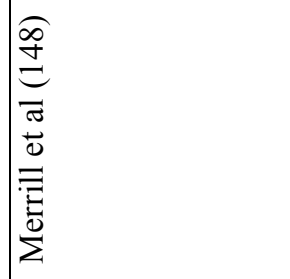 \\
\hline $\begin{array}{l}\dot{z} \\
\dot{z}\end{array}$ & $i$ & $\stackrel{\infty}{2}$ & $\triangleq$ & 8 & $\sqrt{2}$ \\
\hline
\end{tabular}


ล

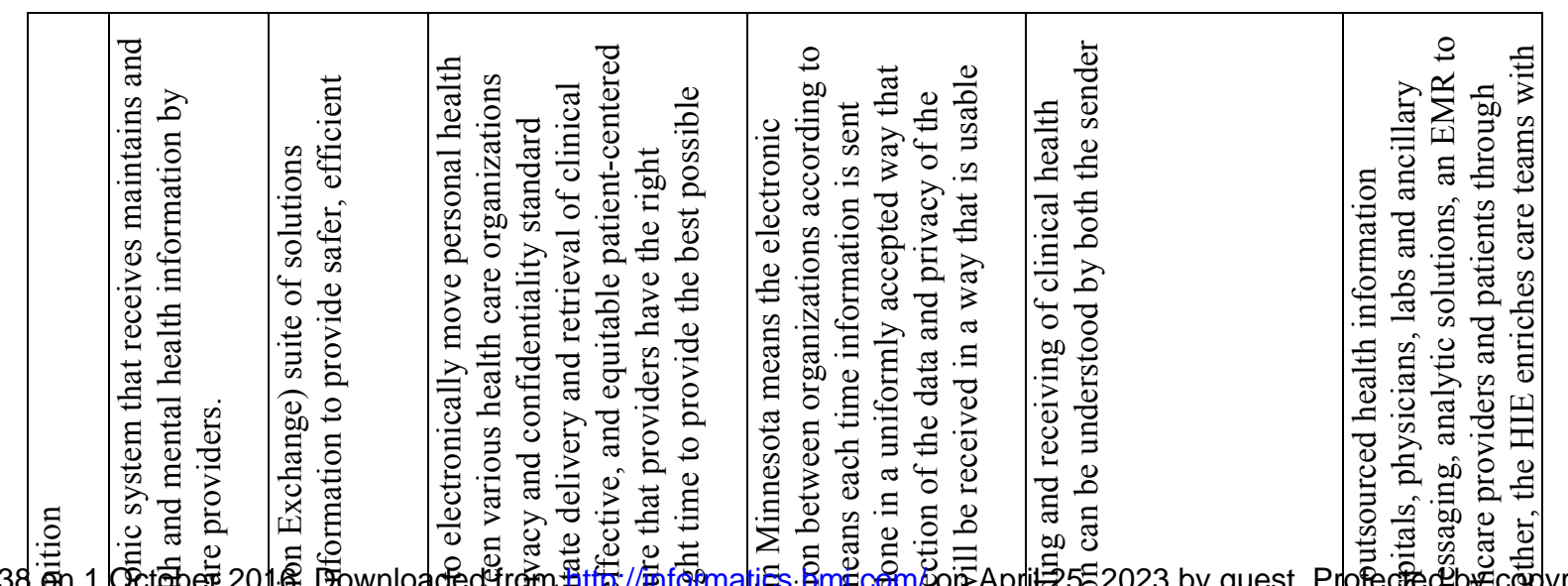

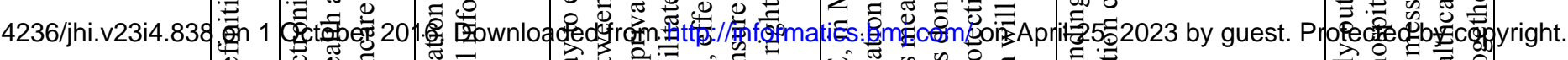

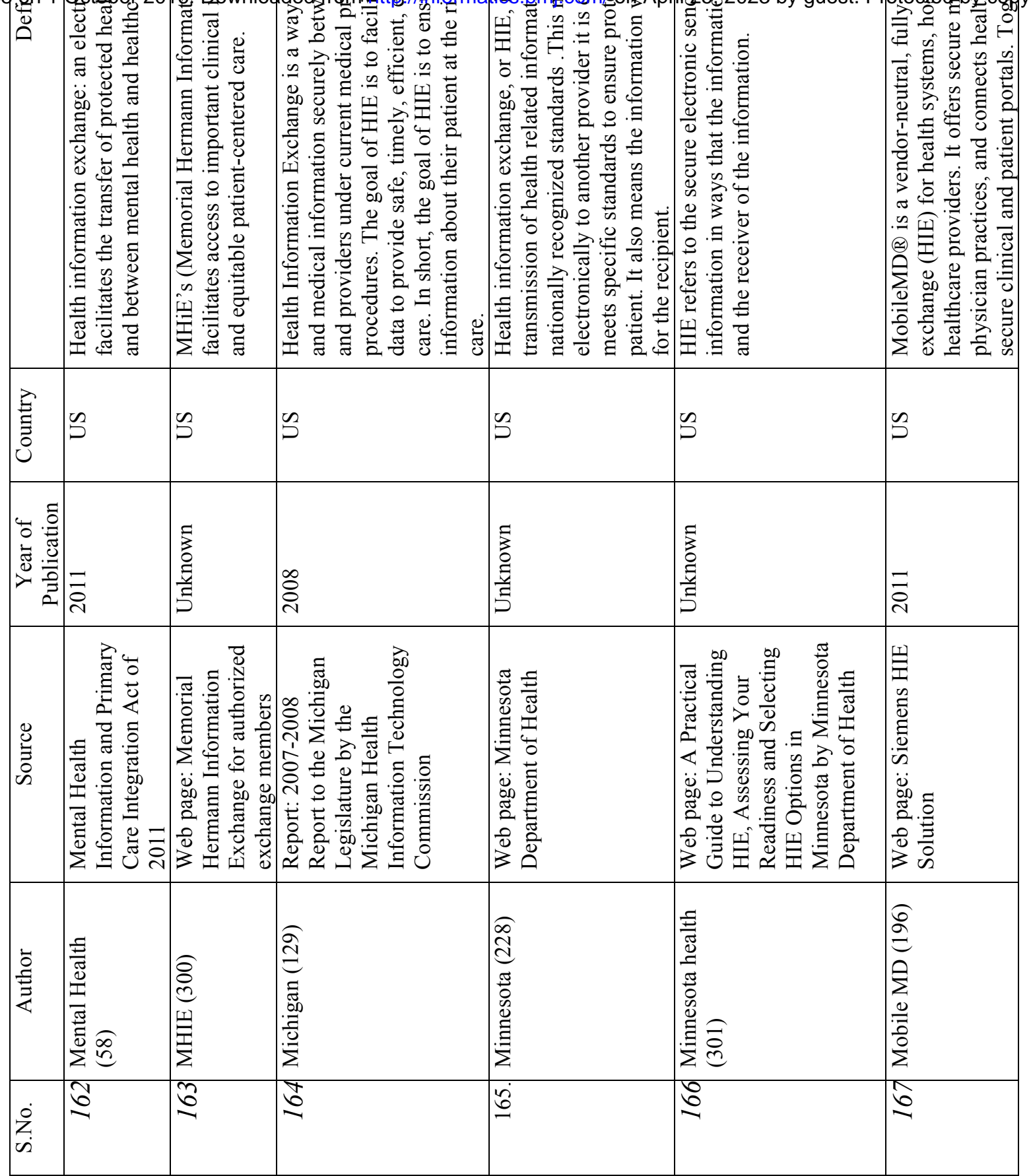


$\stackrel{\infty}{N}$

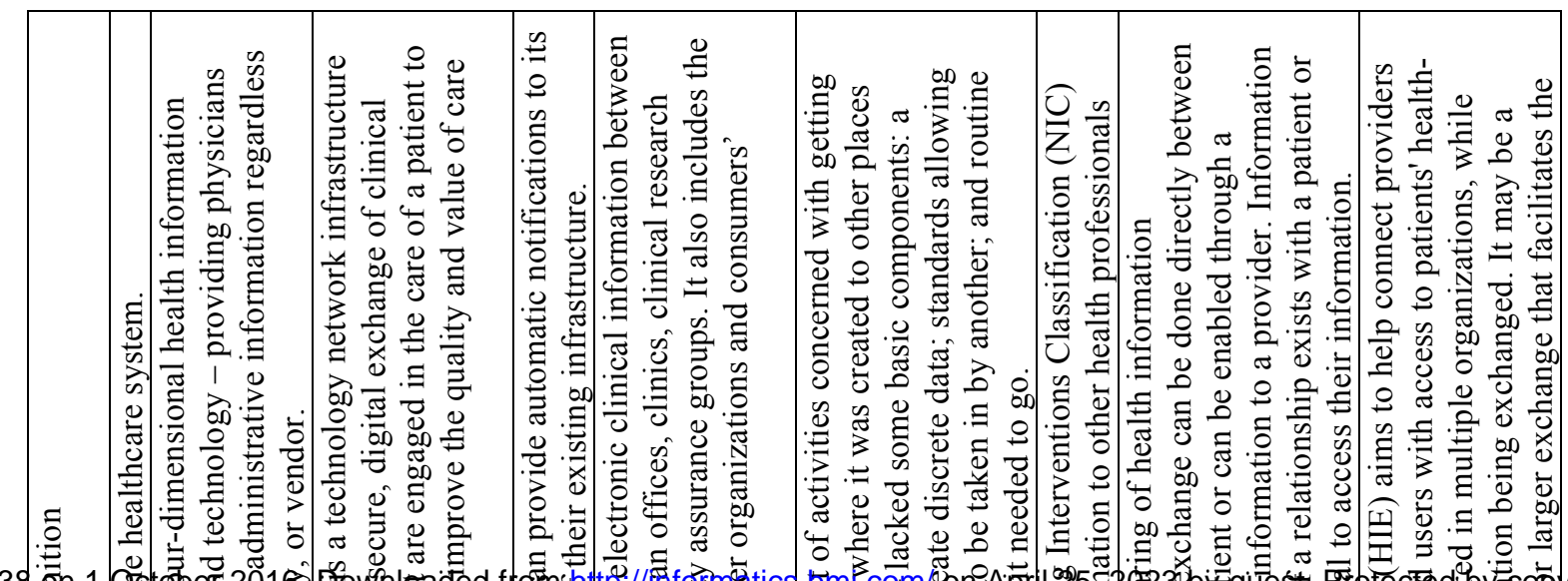

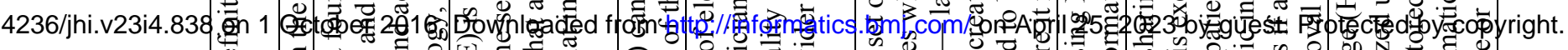

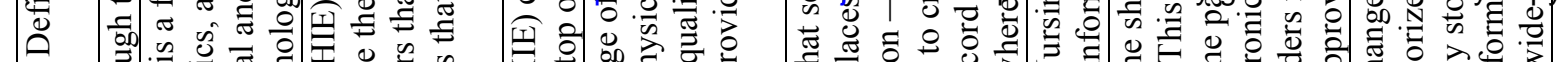

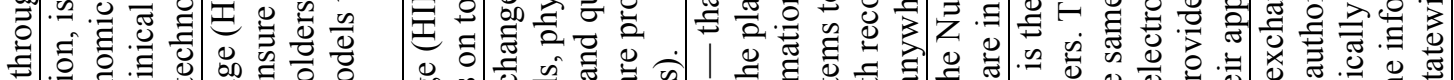

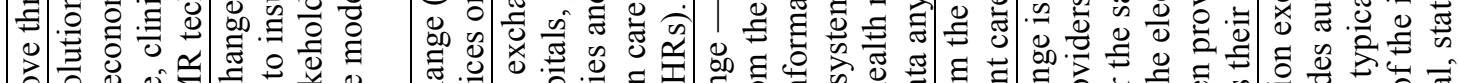

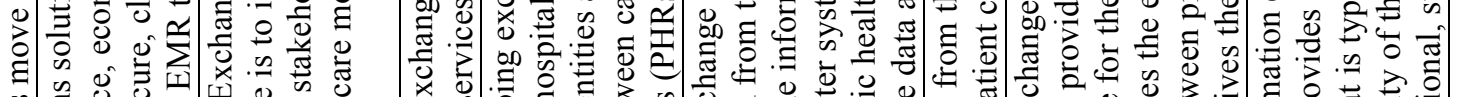

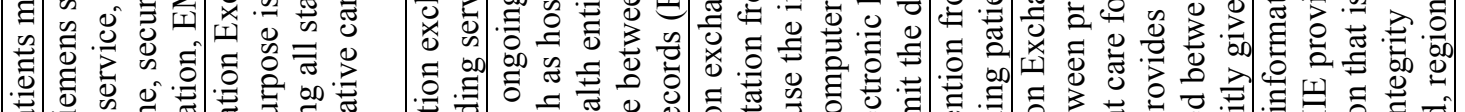

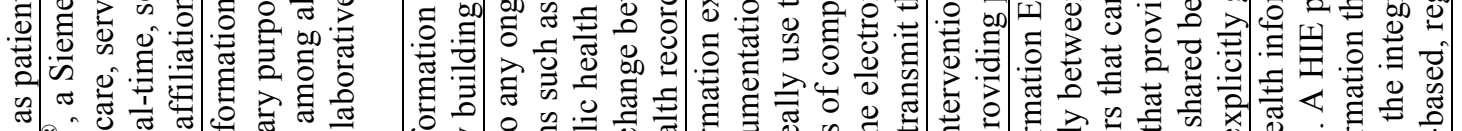

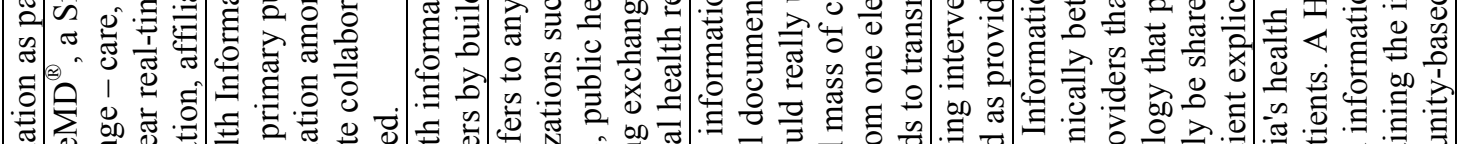

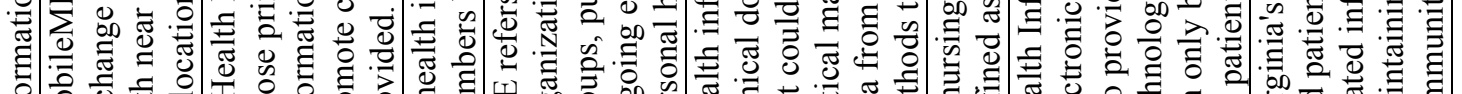

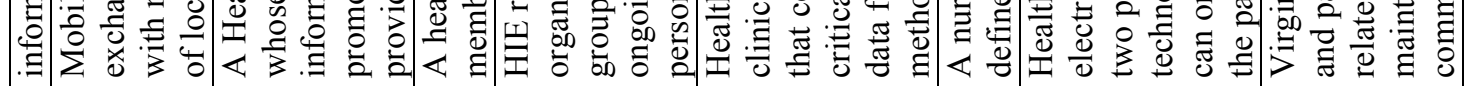

\begin{tabular}{|c|c|c|c|c|c|c|c|c|}
\hline 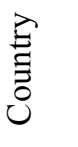 & $\tilde{\rho}$ & $\tilde{\Omega}$ & $\tilde{2}$ & $\begin{array}{l}\frac{3}{0} \\
0 \\
\frac{1}{3} \\
\frac{5}{5}\end{array}$ & $\tilde{s}$ & $\tilde{D}$ & $\tilde{s}$ & $\Omega$ \\
\hline 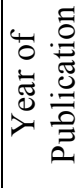 & 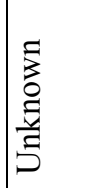 & $\overrightarrow{\vec{d}}$ & $\stackrel{\sim}{\stackrel{\sim}{\sim}}$ & 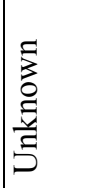 & 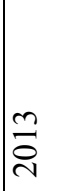 & ஓें & 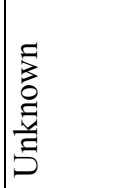 & 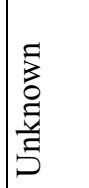 \\
\hline 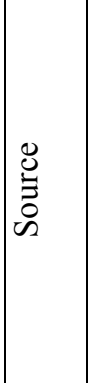 & 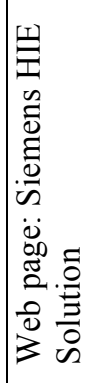 & 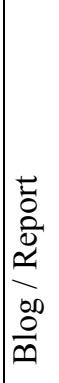 & 氖 & 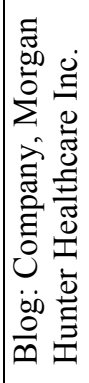 & 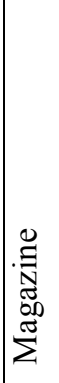 & 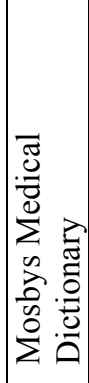 & 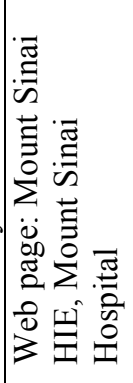 & 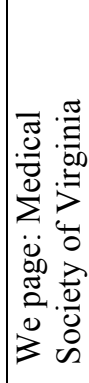 \\
\hline 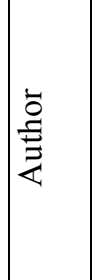 & 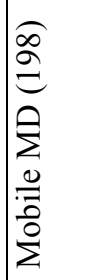 & 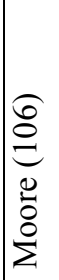 & 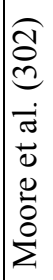 & 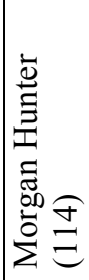 & 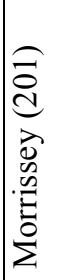 & 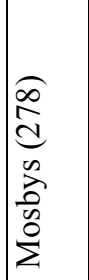 & 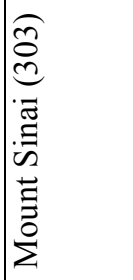 & $\begin{array}{l}\widehat{I} \\
\infty \\
z \\
\infty \\
\Sigma\end{array}$ \\
\hline $\begin{array}{l}\dot{z} \\
\dot{n}\end{array}$ & $\infty$ & $\stackrel{0}{-1}$ & $\cong$ & 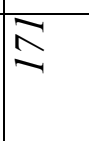 & $\stackrel{N}{=}$ & $\cong$ & $\stackrel{2}{\simeq}$ & 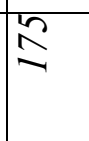 \\
\hline
\end{tabular}


ลิ

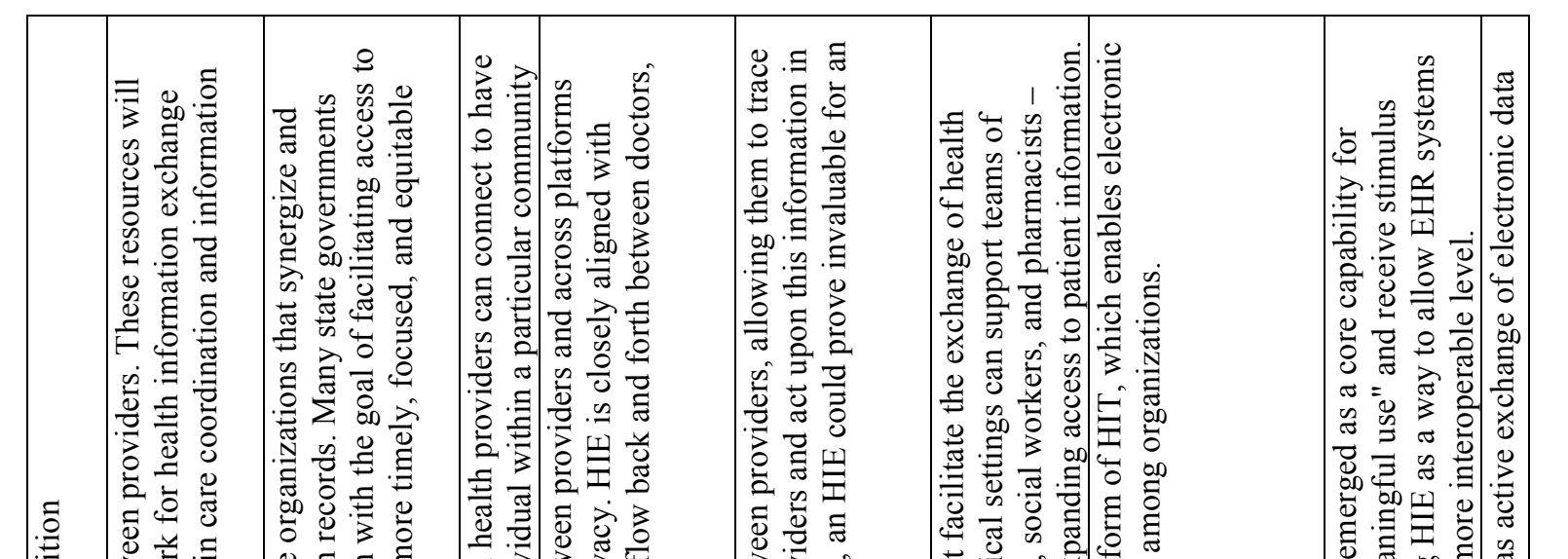

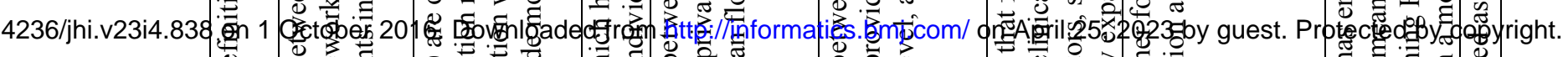

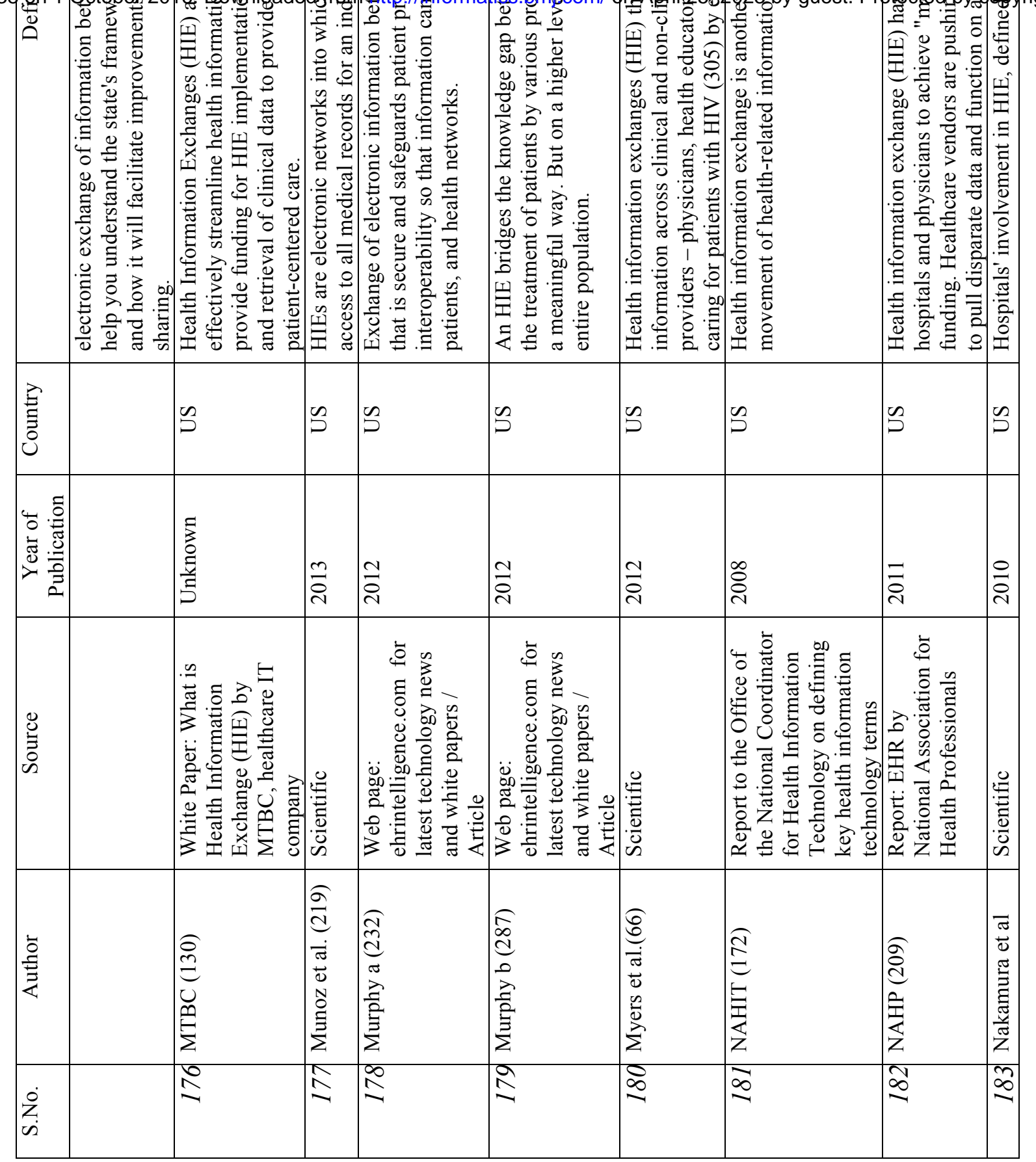


욧

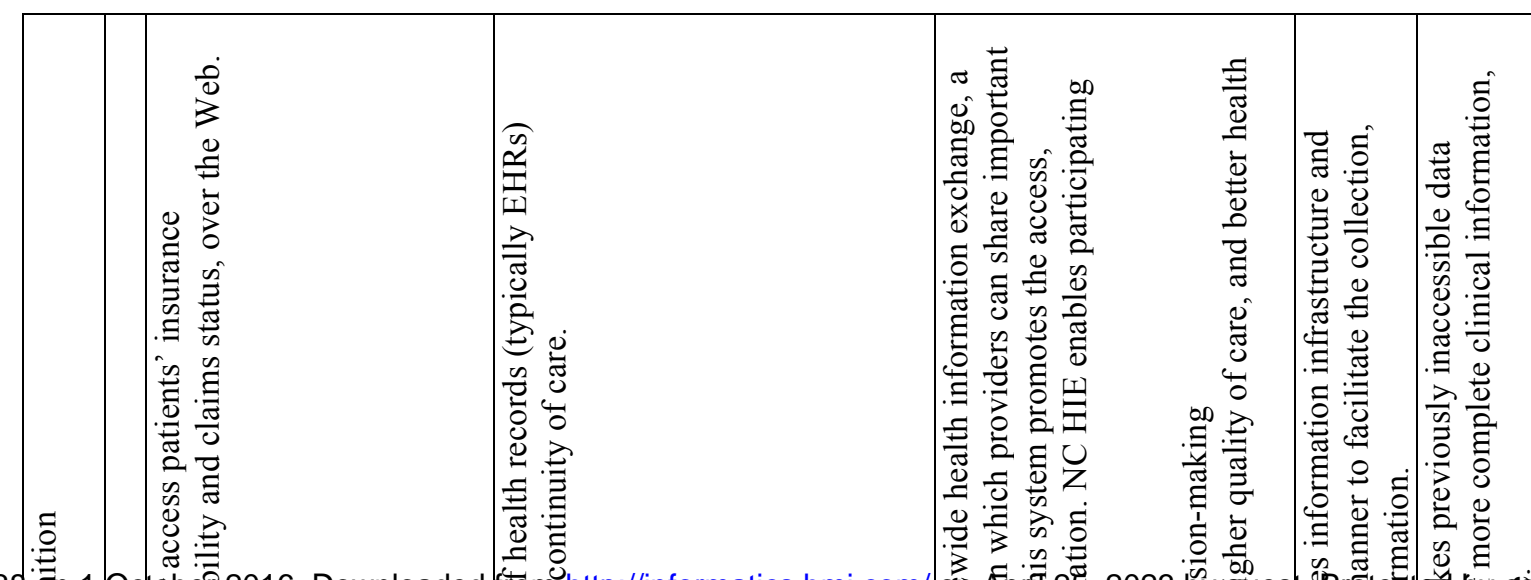

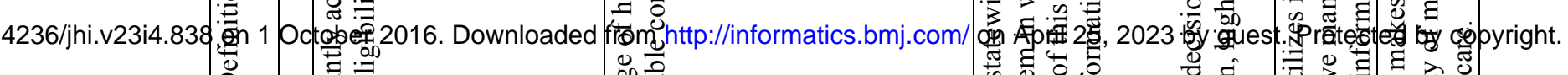

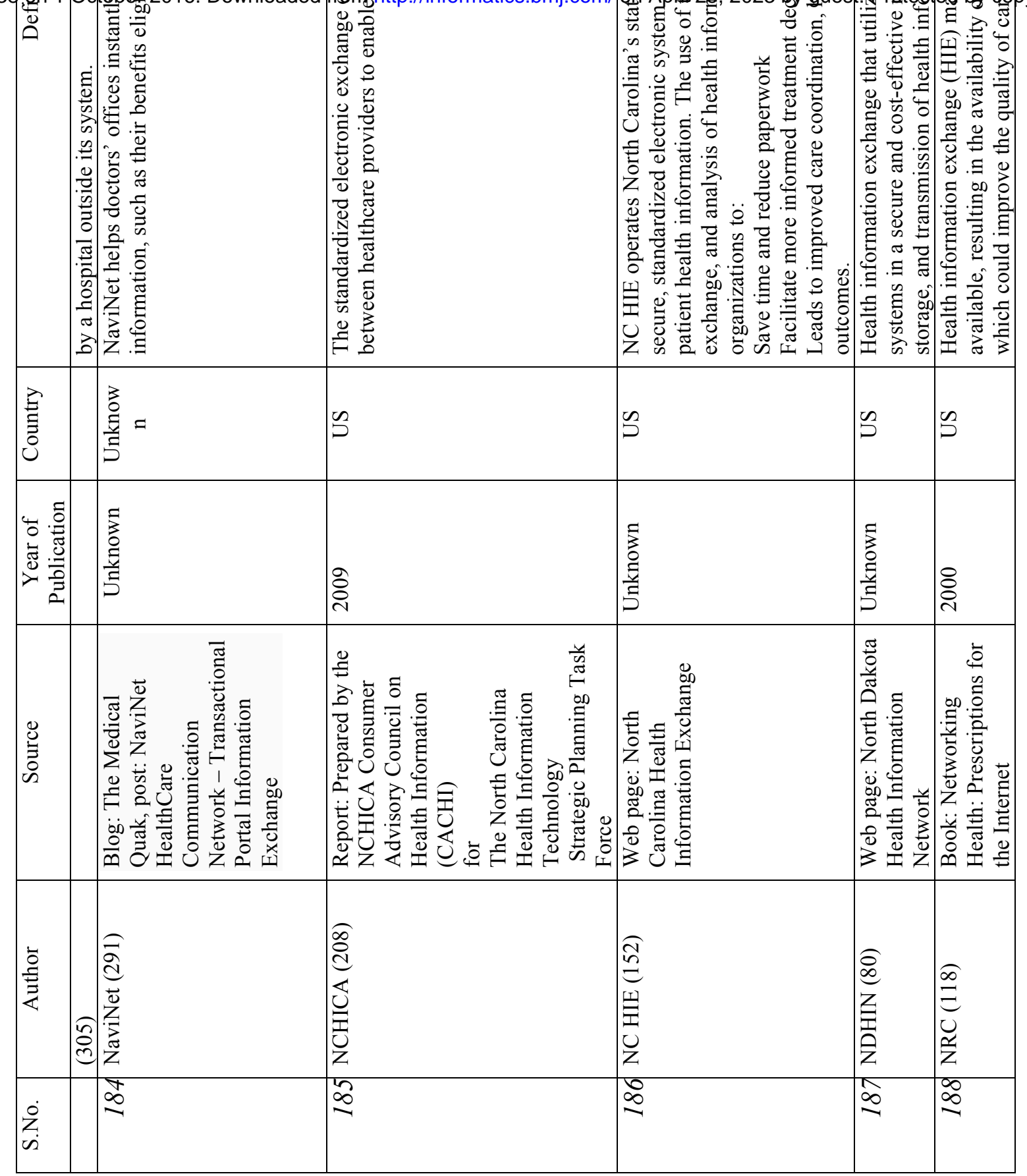


$\vec{m}$

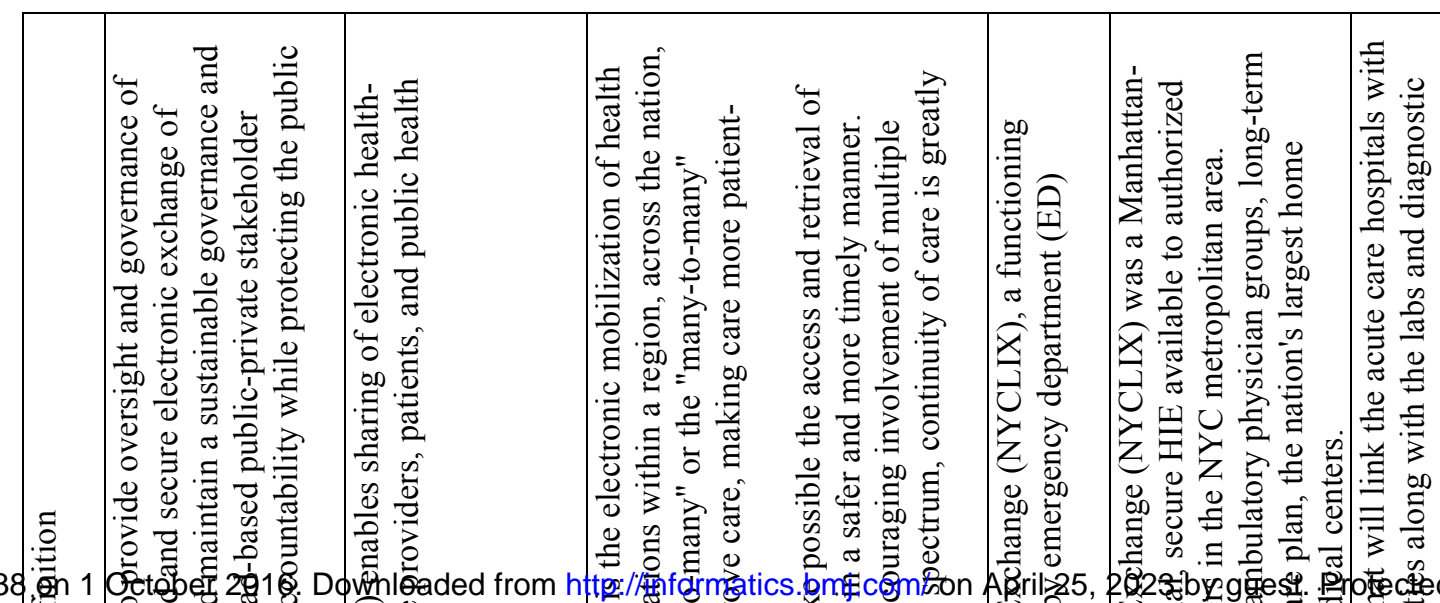

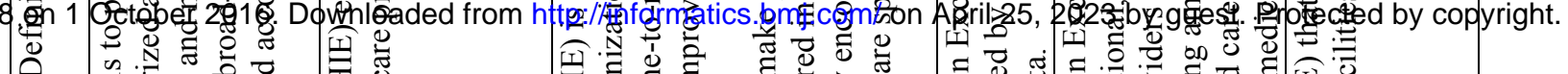

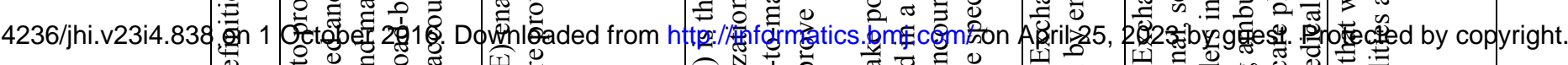

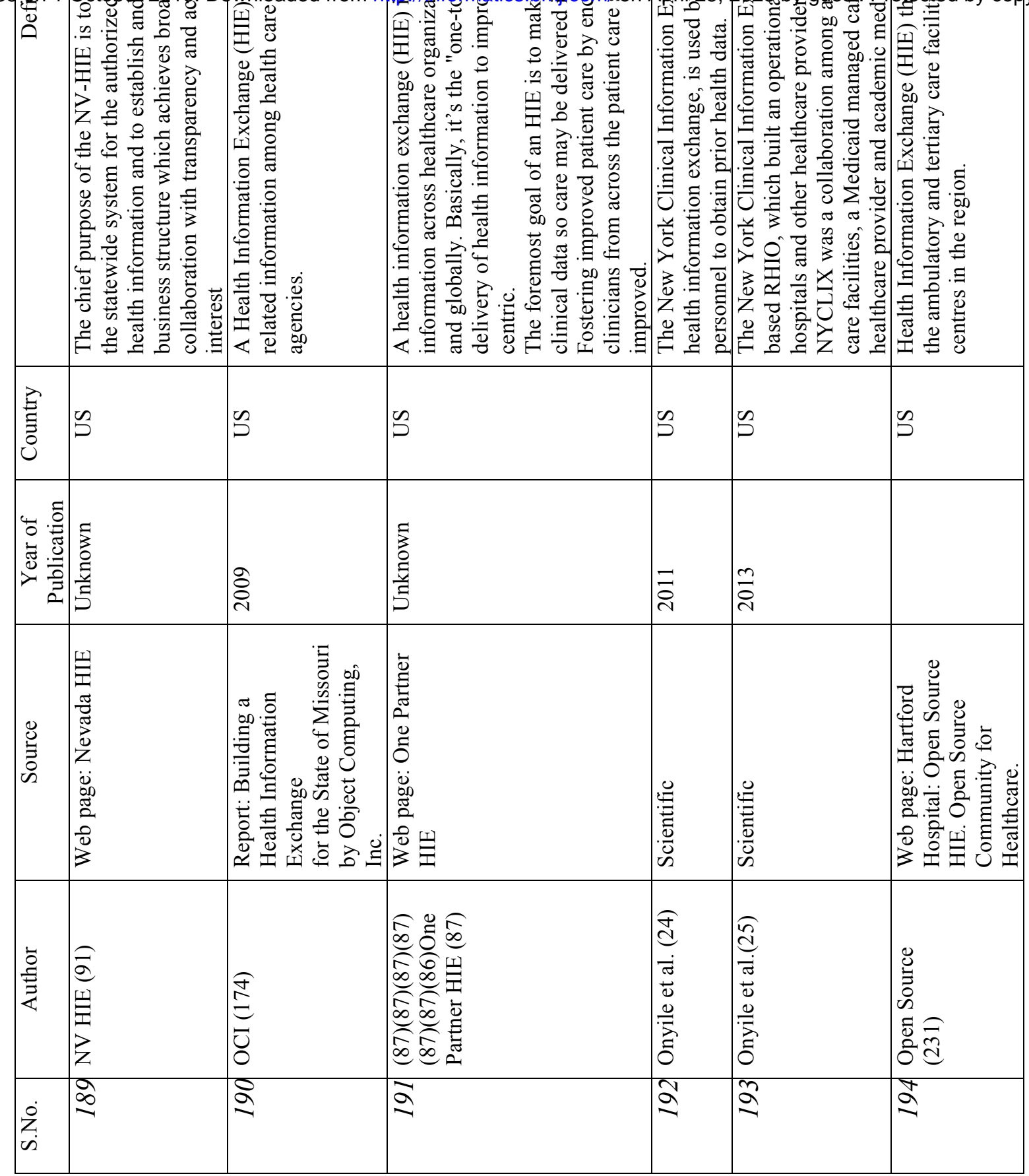


ก

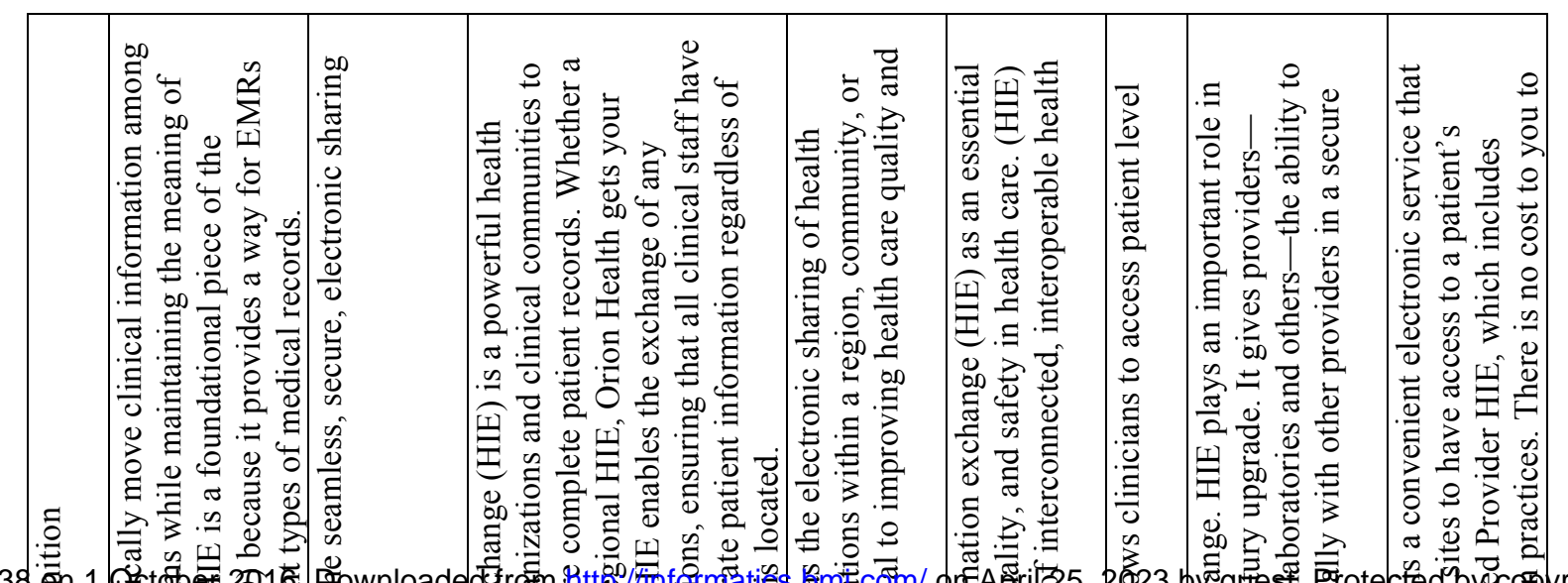

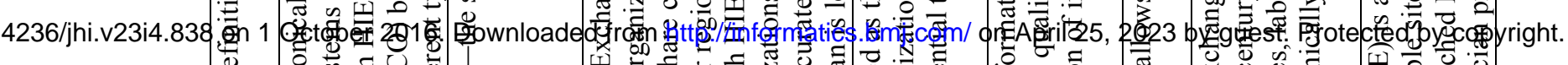

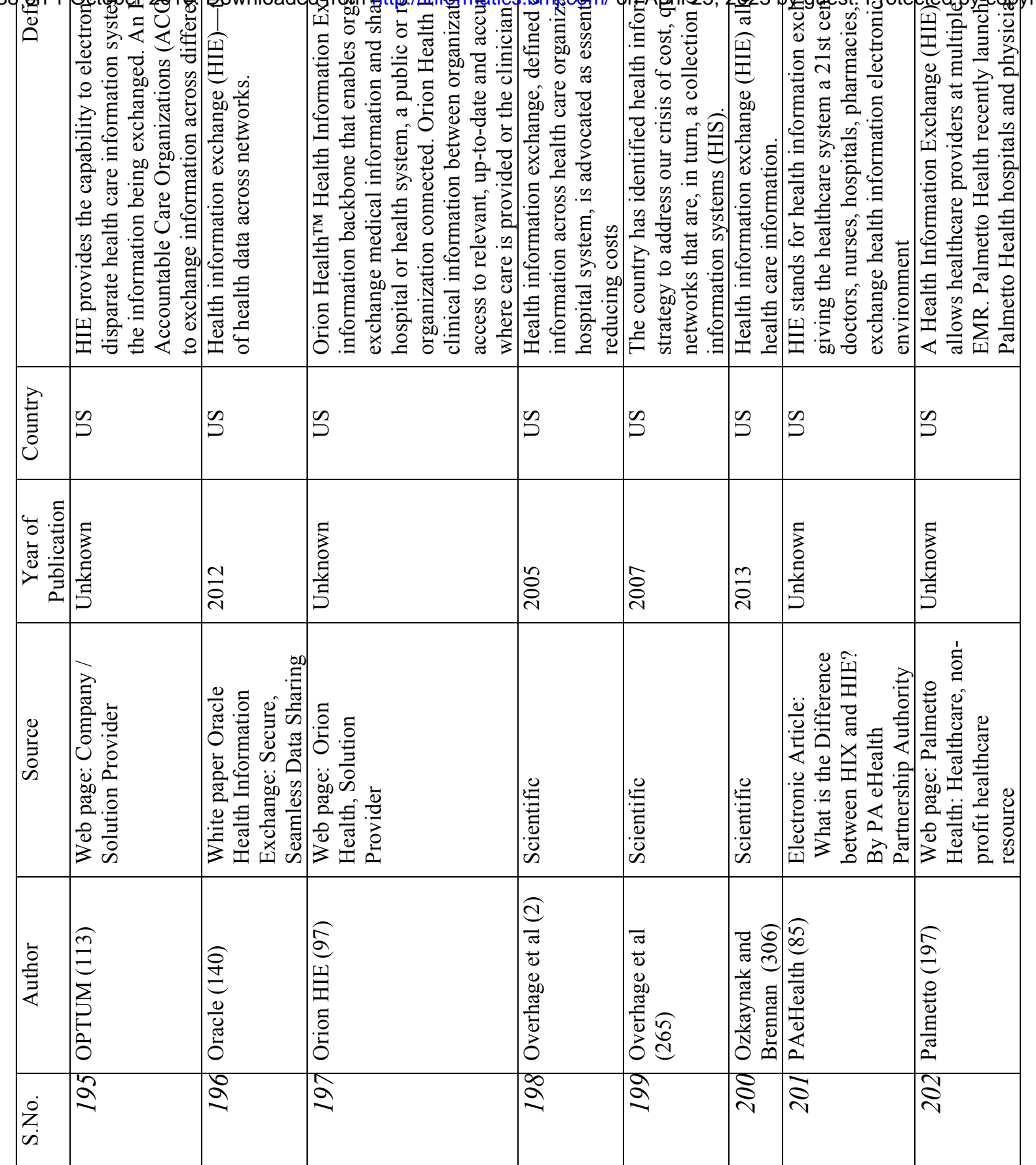


$\stackrel{m}{N}$

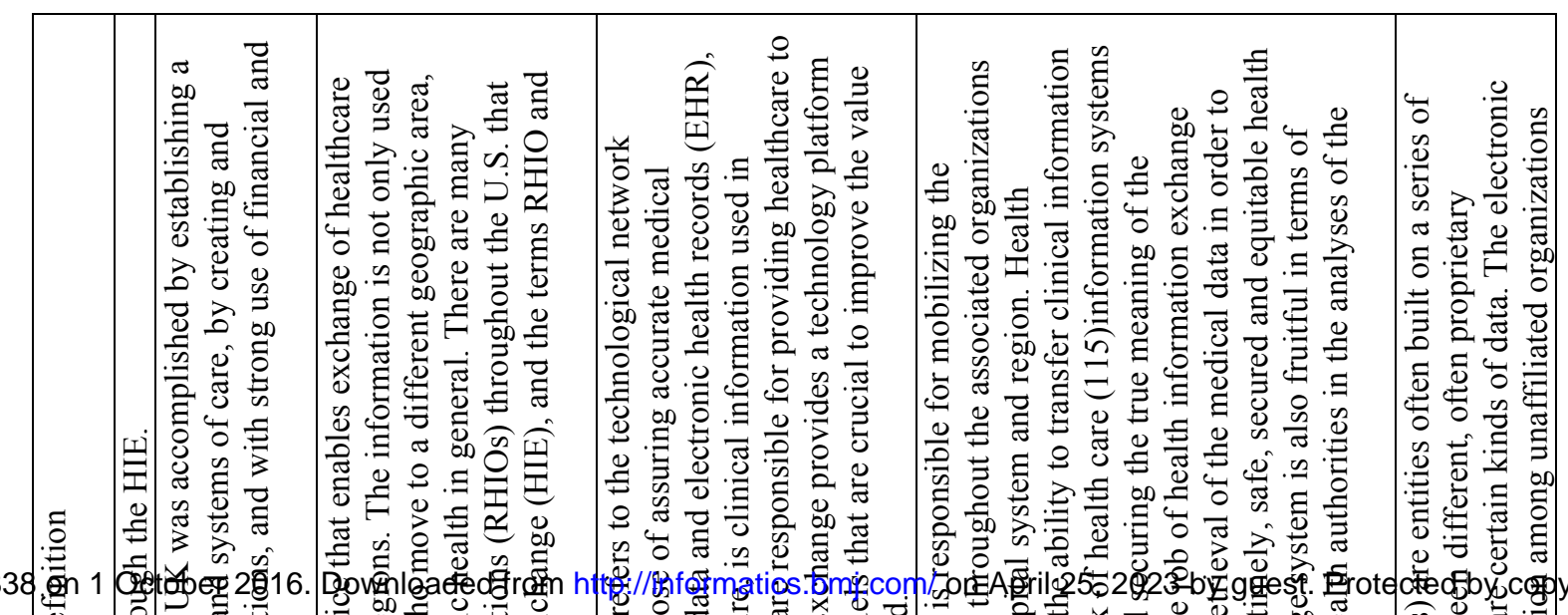

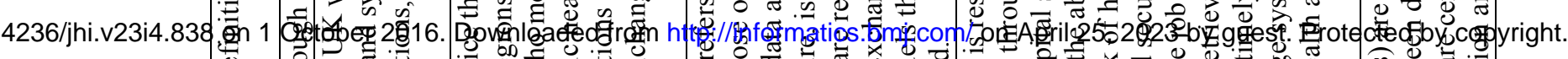

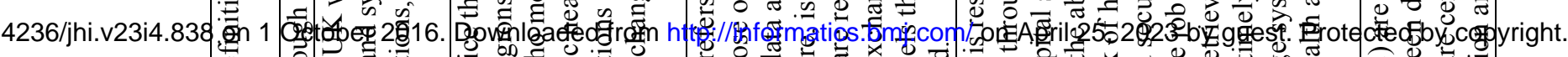

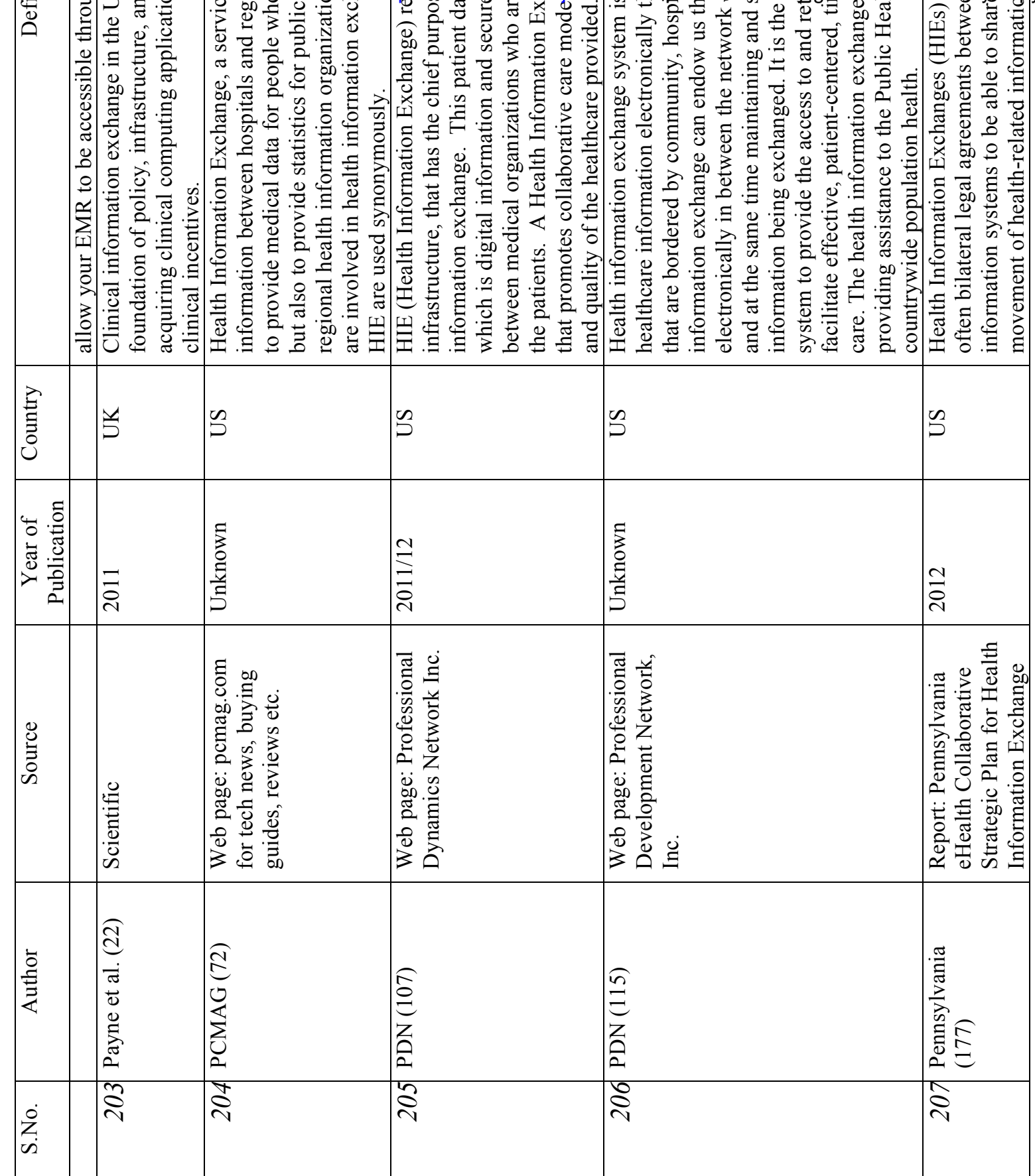


品

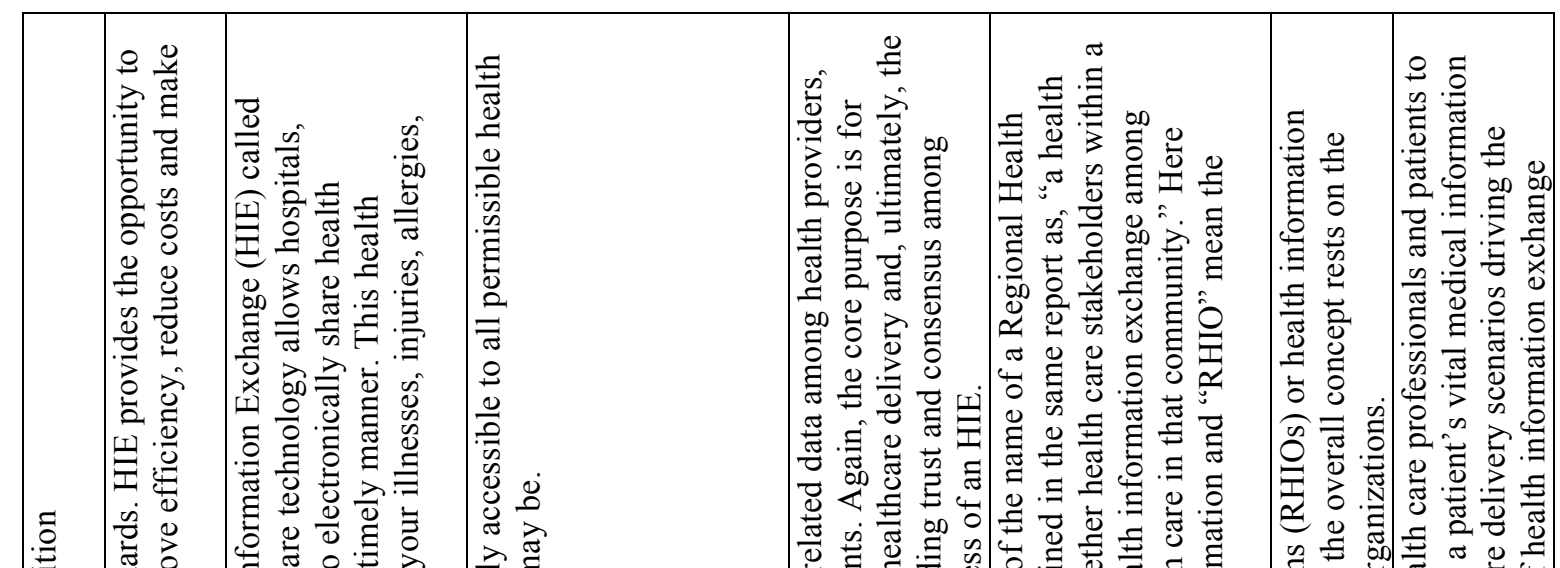

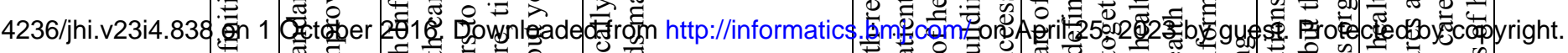

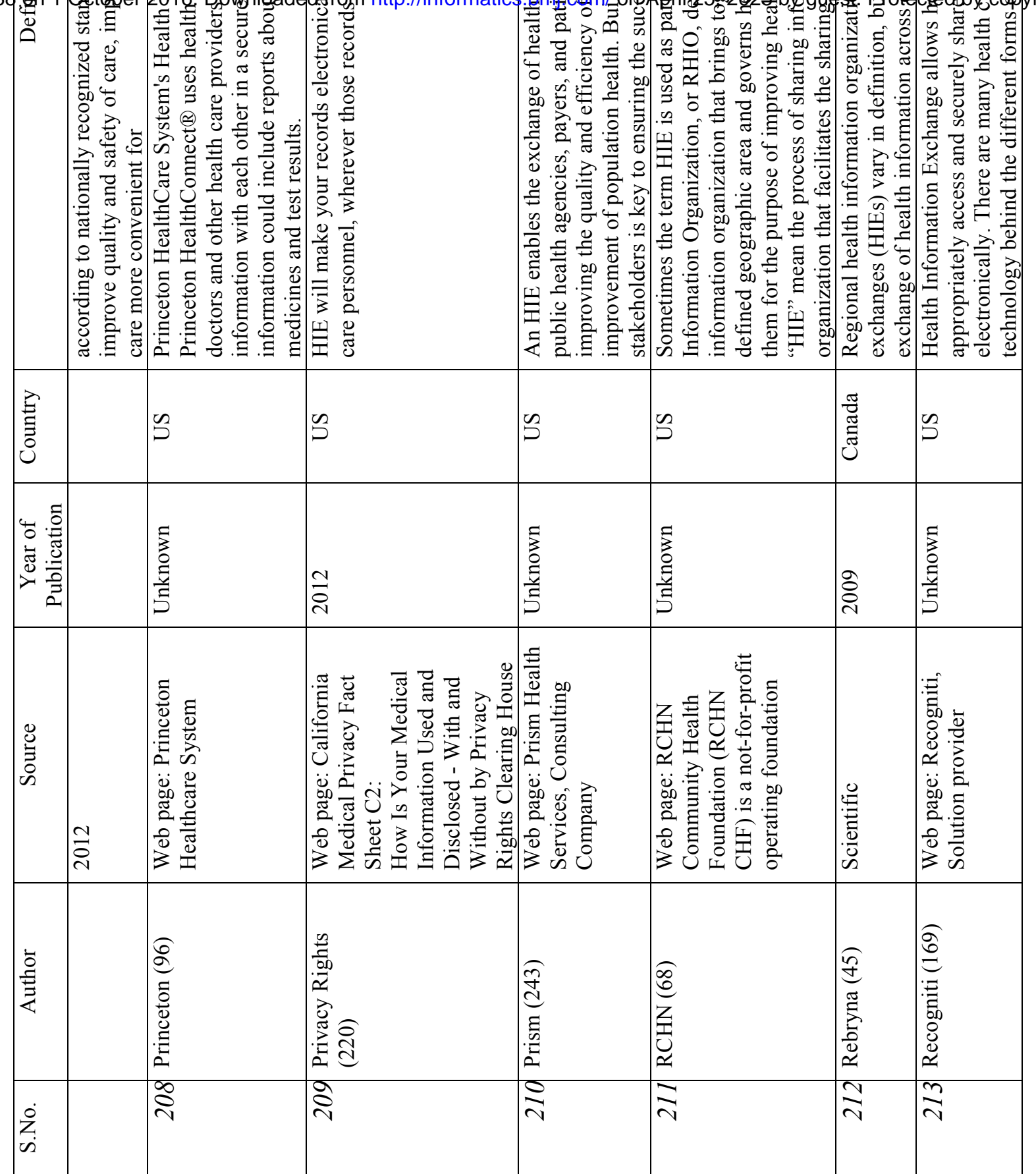


$\stackrel{\leftrightarrow}{\stackrel{n}{n}}$

\begin{tabular}{|c|c|c|c|c|c|c|c|c|c|}
\hline & 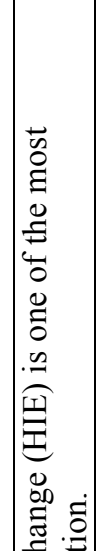 & 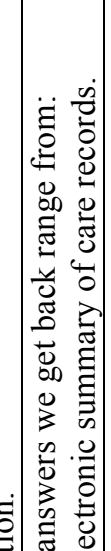 & 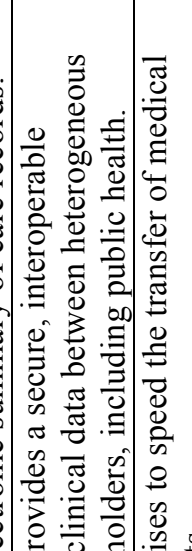 & 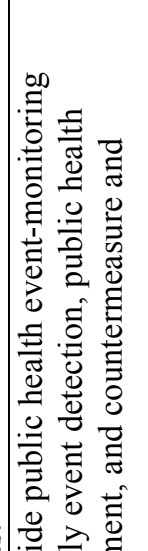 & 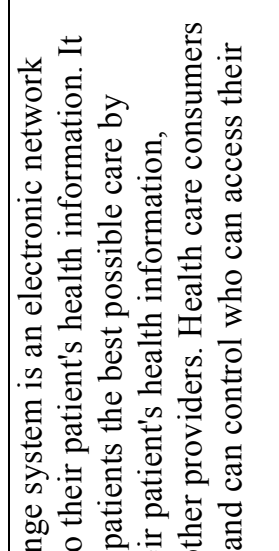 & 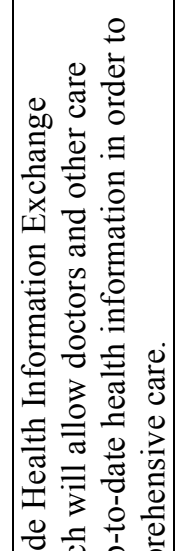 & 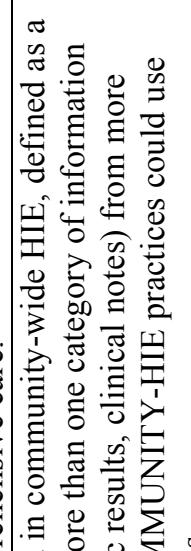 & 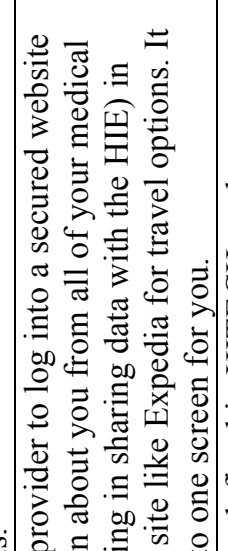 & 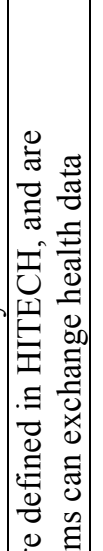 \\
\hline
\end{tabular}

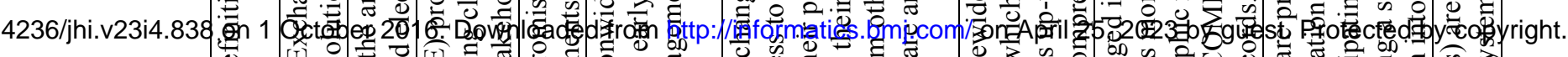

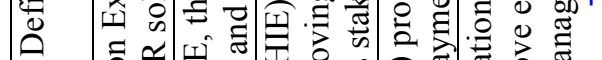
एँ .气

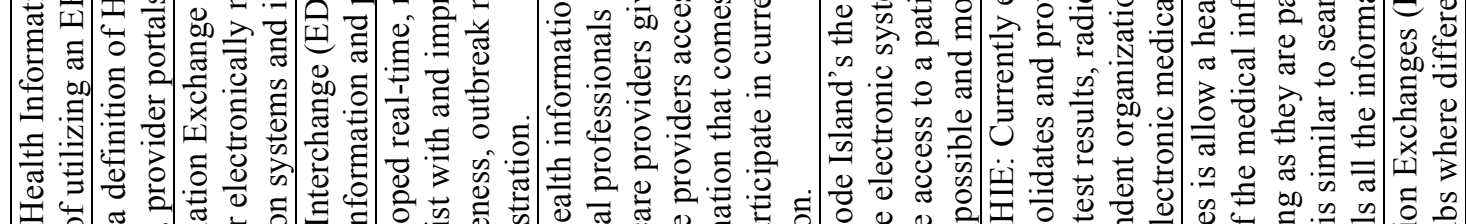

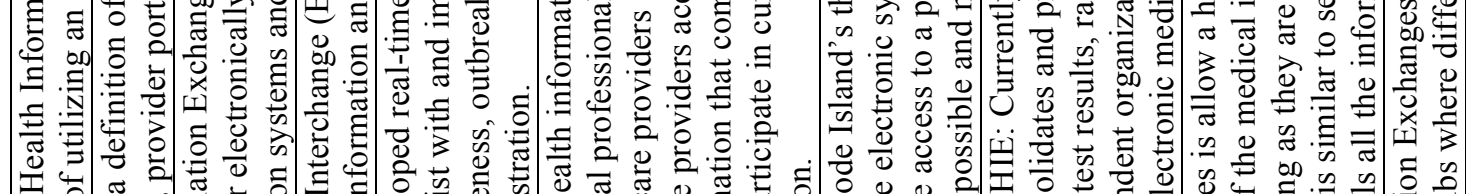

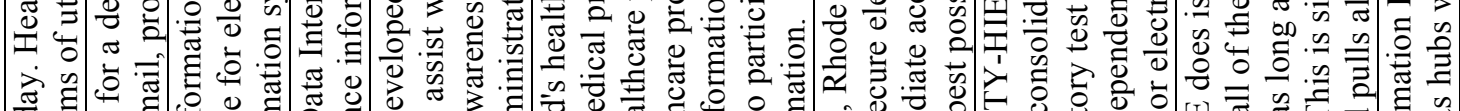

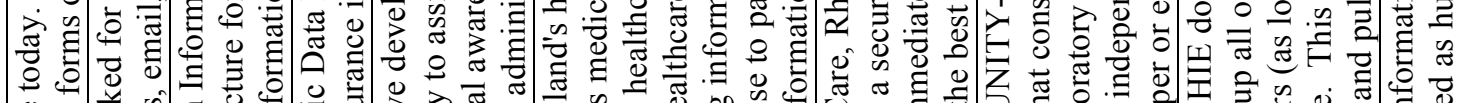

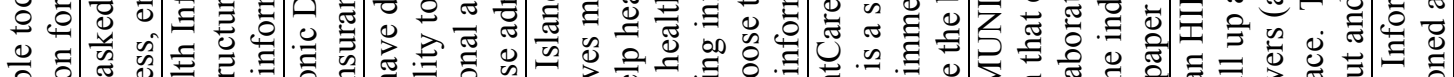
考 焉

\begin{tabular}{|c|c|c|c|c|c|c|c|c|c|}
\hline 兽 & $\tilde{s}$ & $\stackrel{\sim}{\Omega}$ & 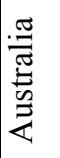 & $\stackrel{\sim}{\Omega}$ & $\mathscr{\sim}$ & $\tilde{\rho}$ & $\stackrel{\sim}{\Omega}$ & $\tilde{\Omega}$ & $\mathscr{\Omega}$ \\
\hline 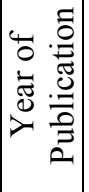 & 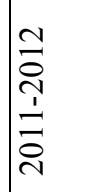 & $\stackrel{\stackrel{N}{\sim}}{\stackrel{\sim}{\sim}}$ & $\bar{\sigma}$ & $\stackrel{\circ}{\circ}$ & 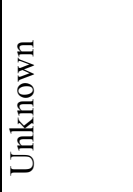 & 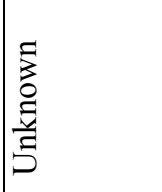 & 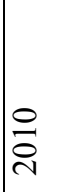 & $\frac{m}{\vec{N}}$ & $\frac{m}{\stackrel{D}{d}}$ \\
\hline 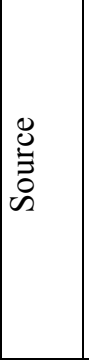 & 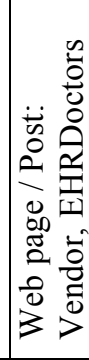 & 兽 & 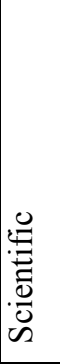 & 总 & 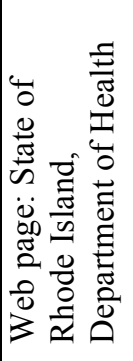 & 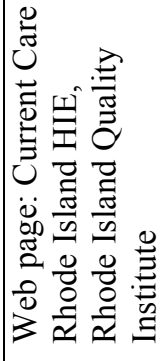 & 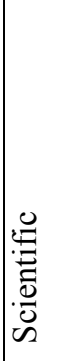 & 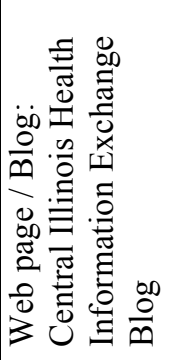 & 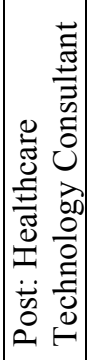 \\
\hline 矛 & 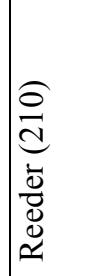 & 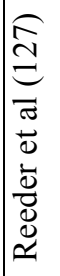 & 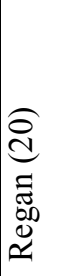 & 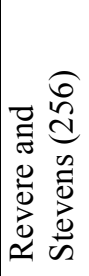 & 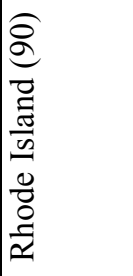 & 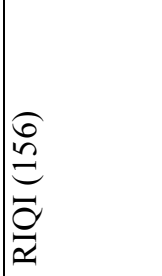 & 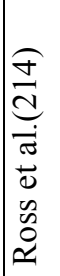 & 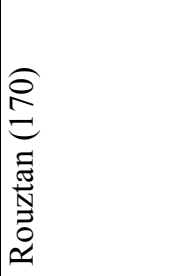 & 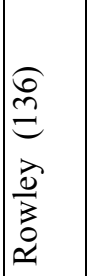 \\
\hline$z_{0}^{0}$ & $\frac{\nabla}{\approx}$ & $\lesssim$ & $\stackrel{\diamond}{\approx}$ & $\widehat{\nwarrow}$ & $\frac{\infty}{\approx}$ & $\frac{\partial}{\lambda}$ & $\stackrel{\curvearrowright}{\curvearrowright}$ & $\bar{\approx}$ & $\widetilde{\mho}$ \\
\hline
\end{tabular}


$\stackrel{\mathscr{N}}{n}$

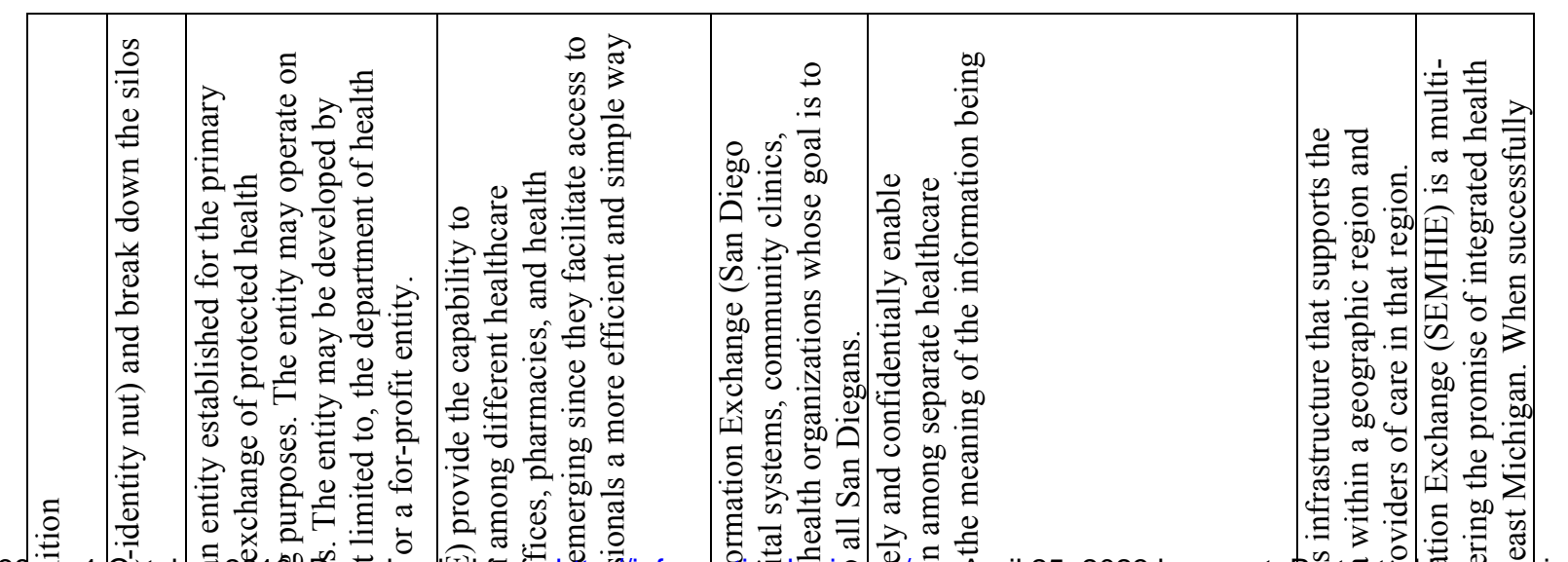

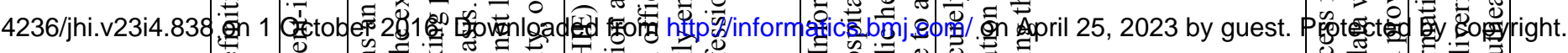

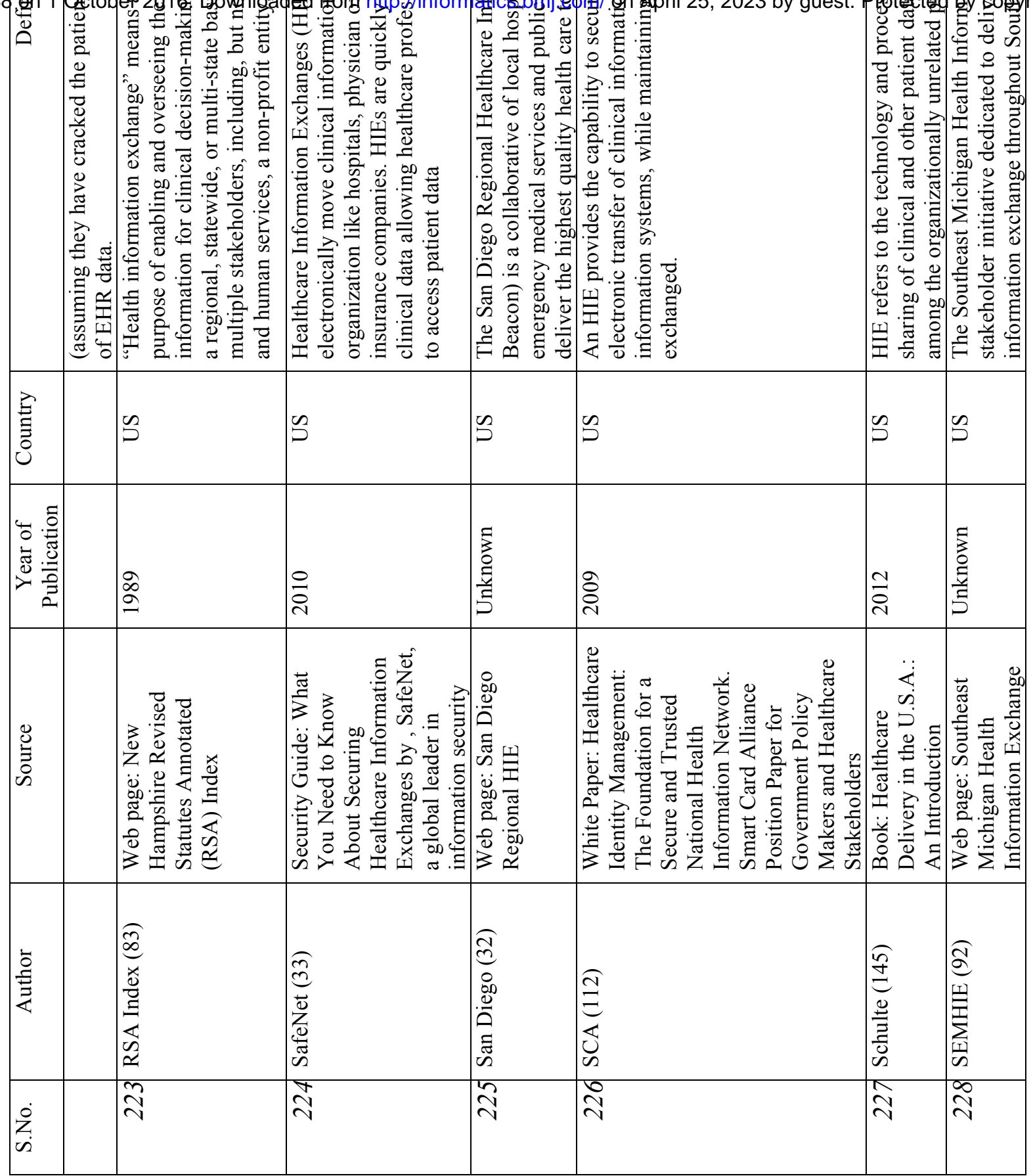


ल

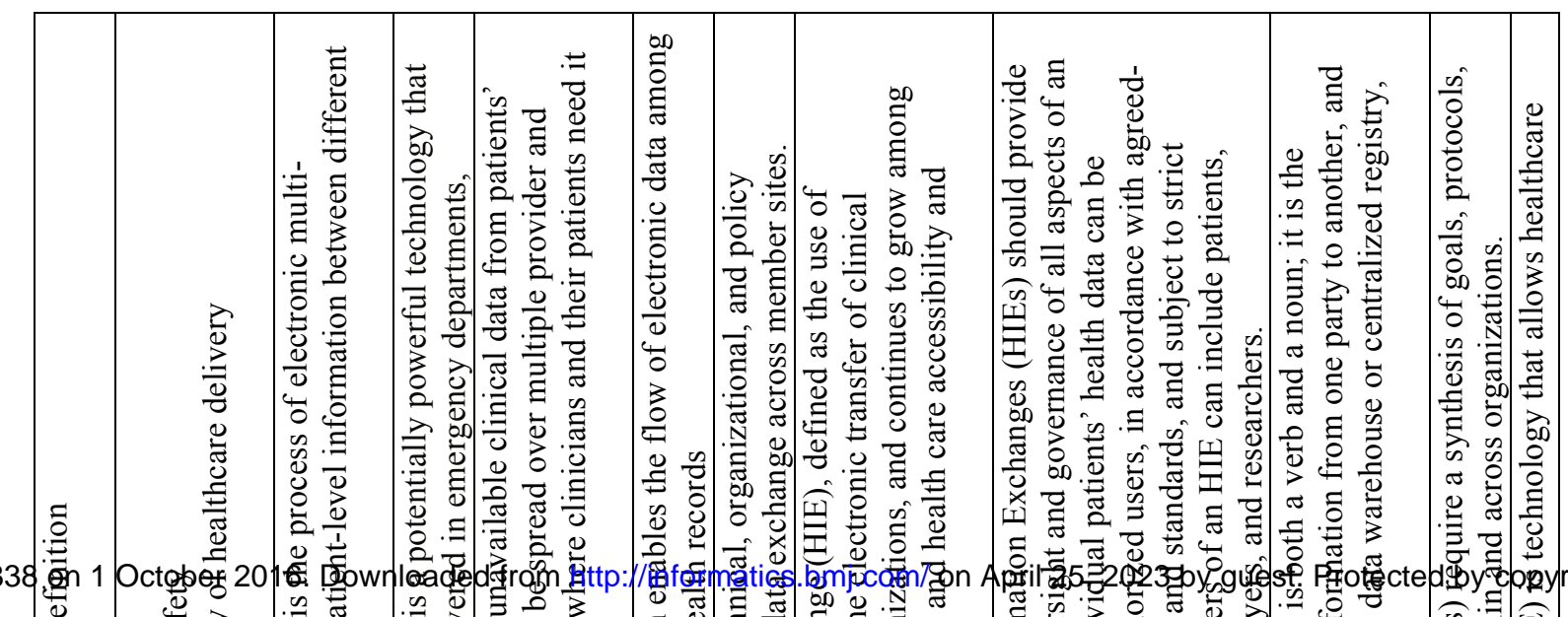

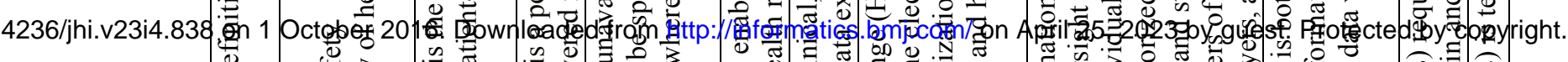

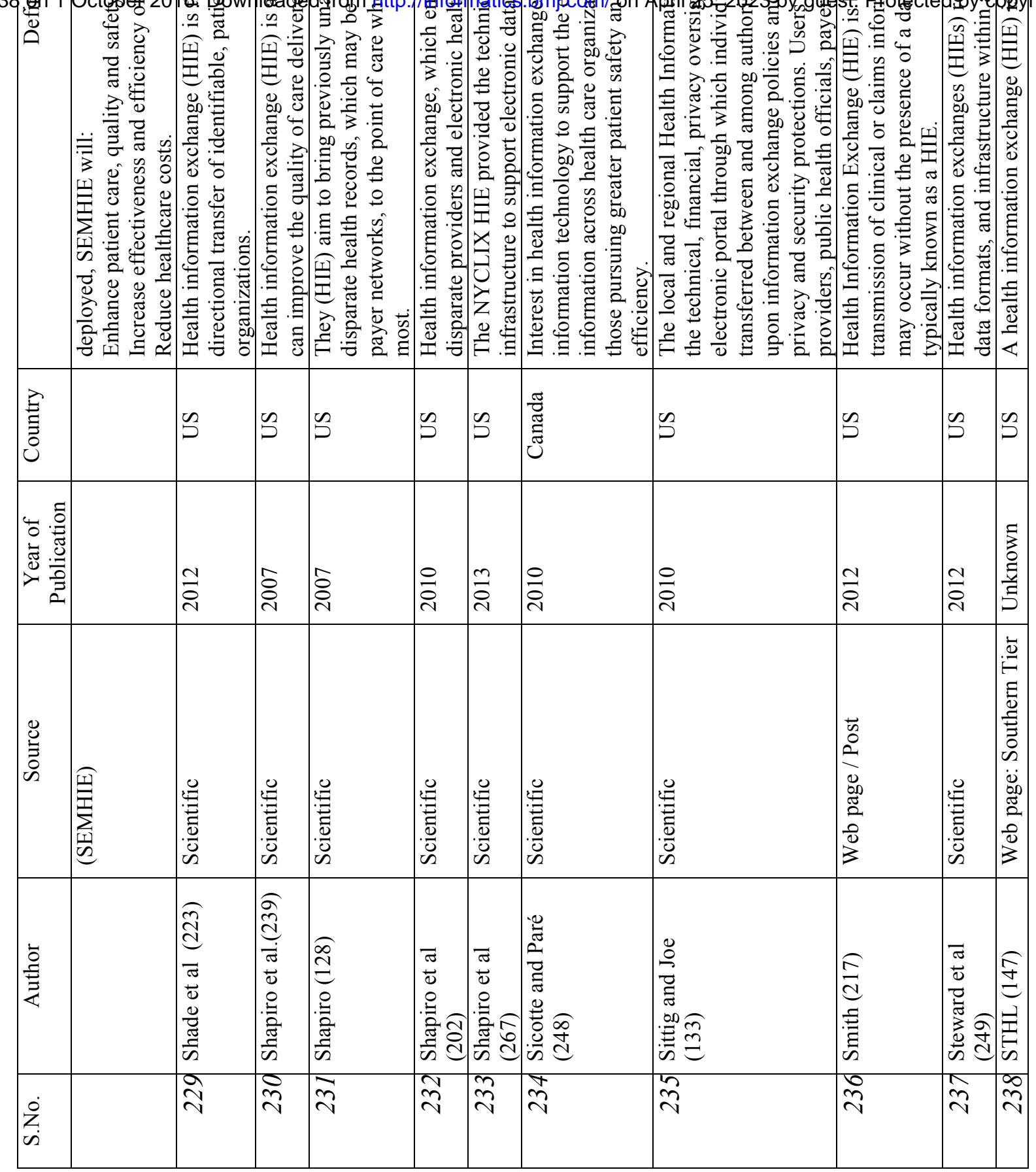


$\stackrel{\infty}{N}$

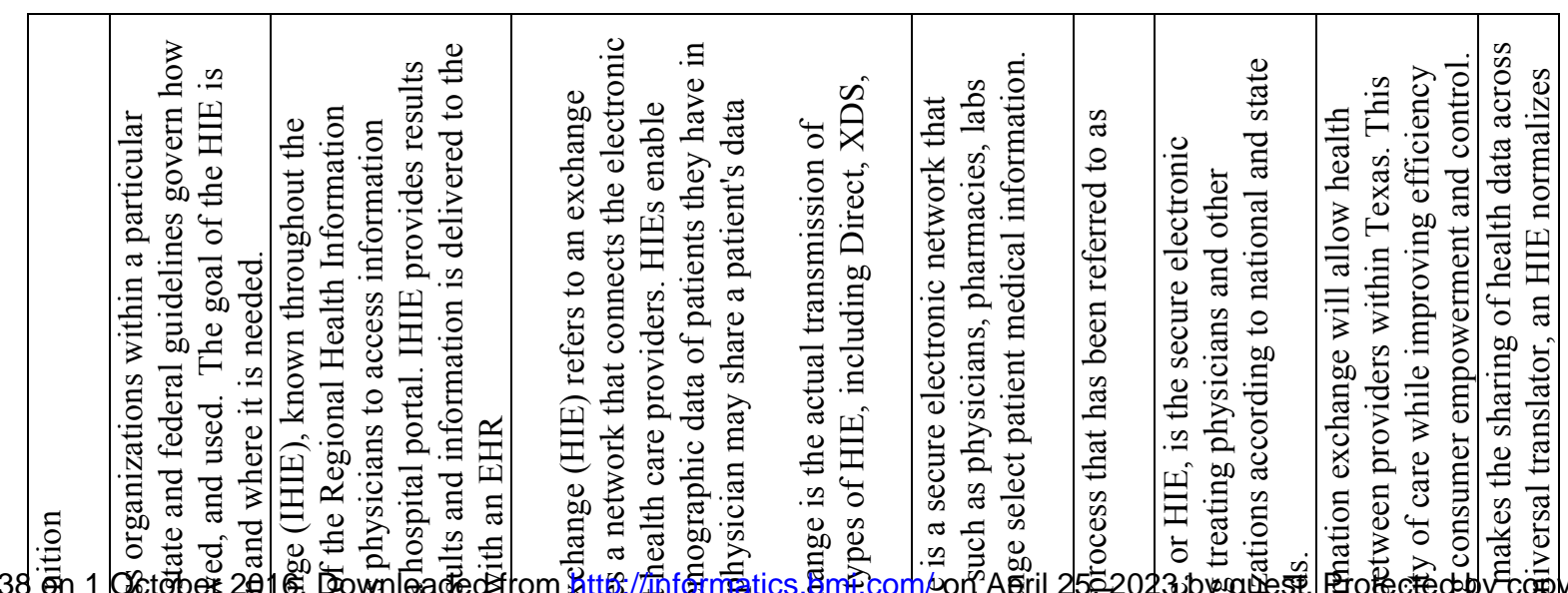

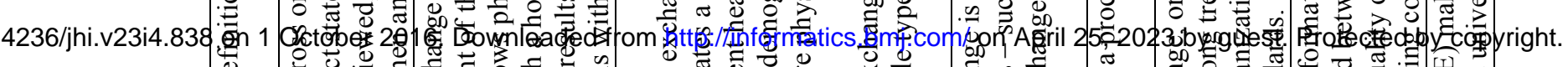

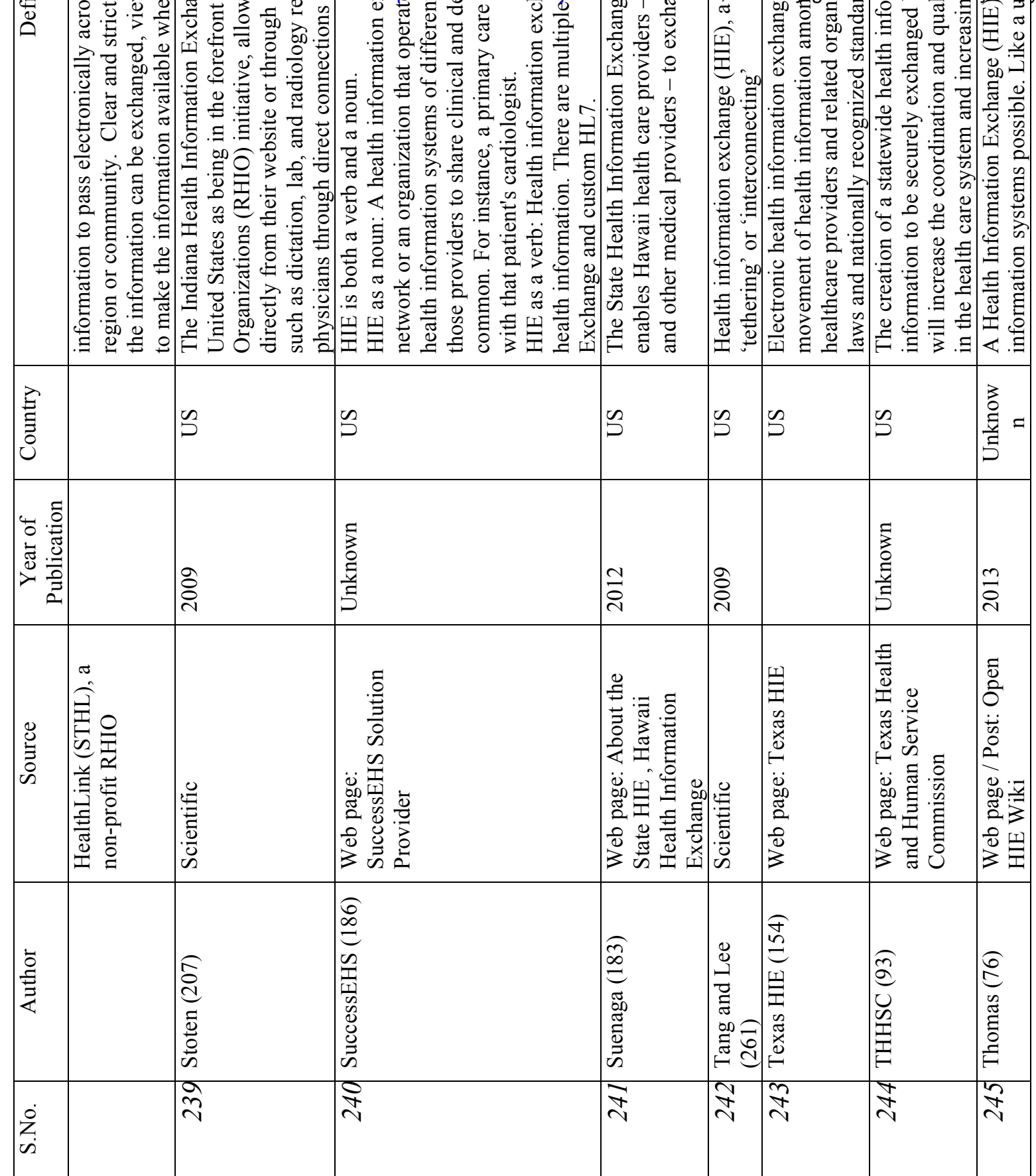


요

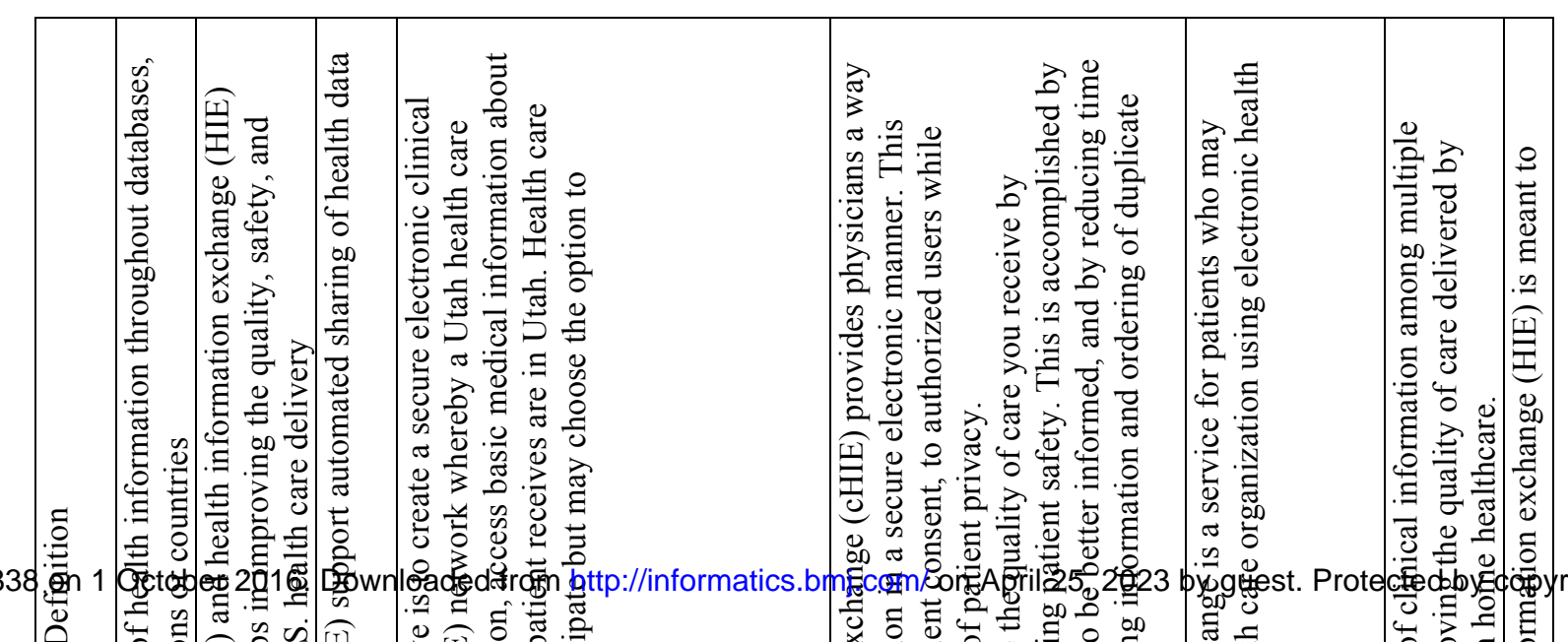

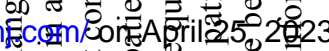

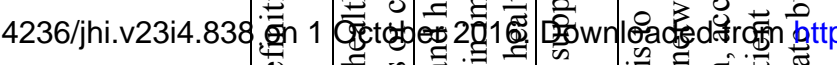

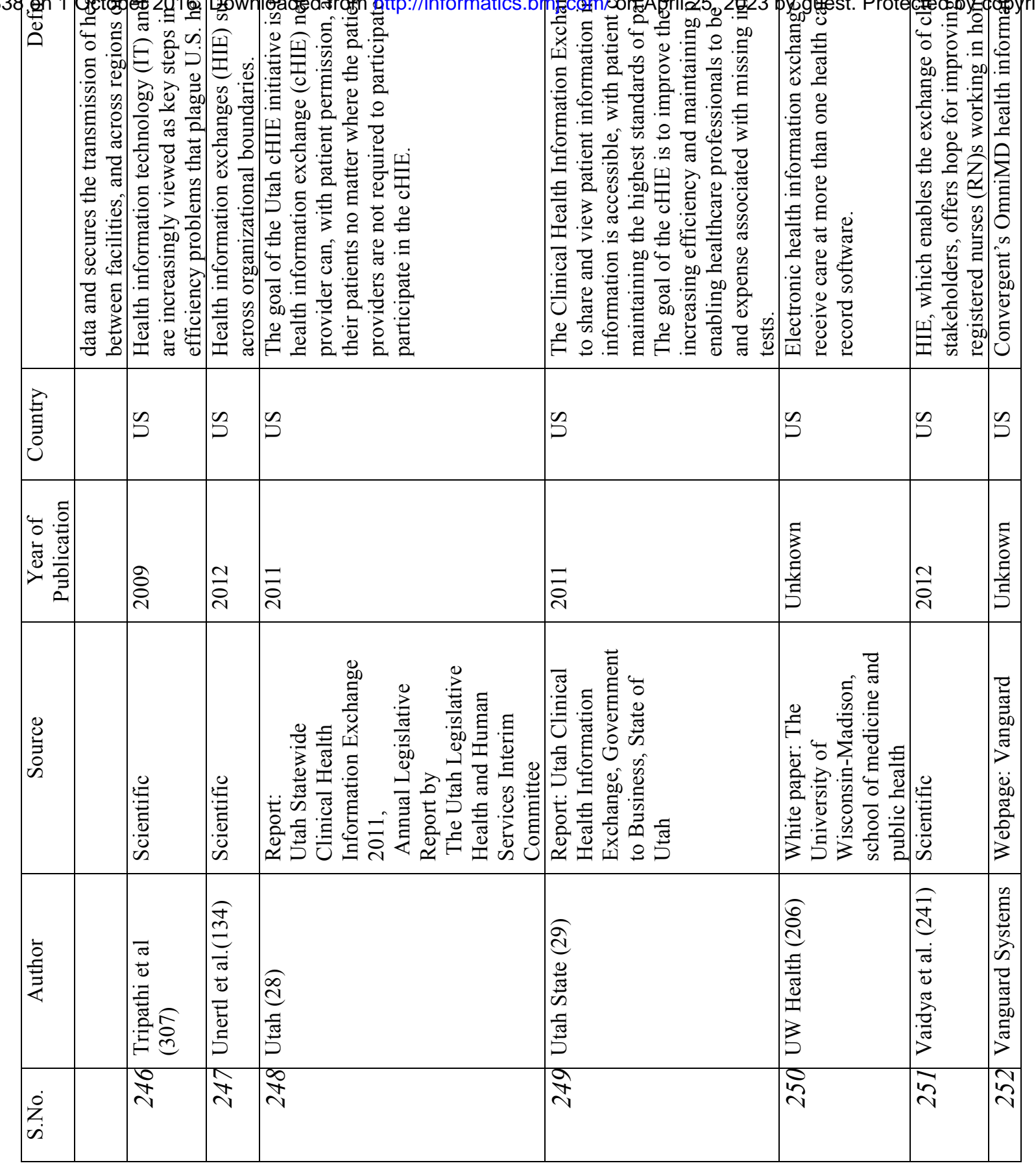


욤

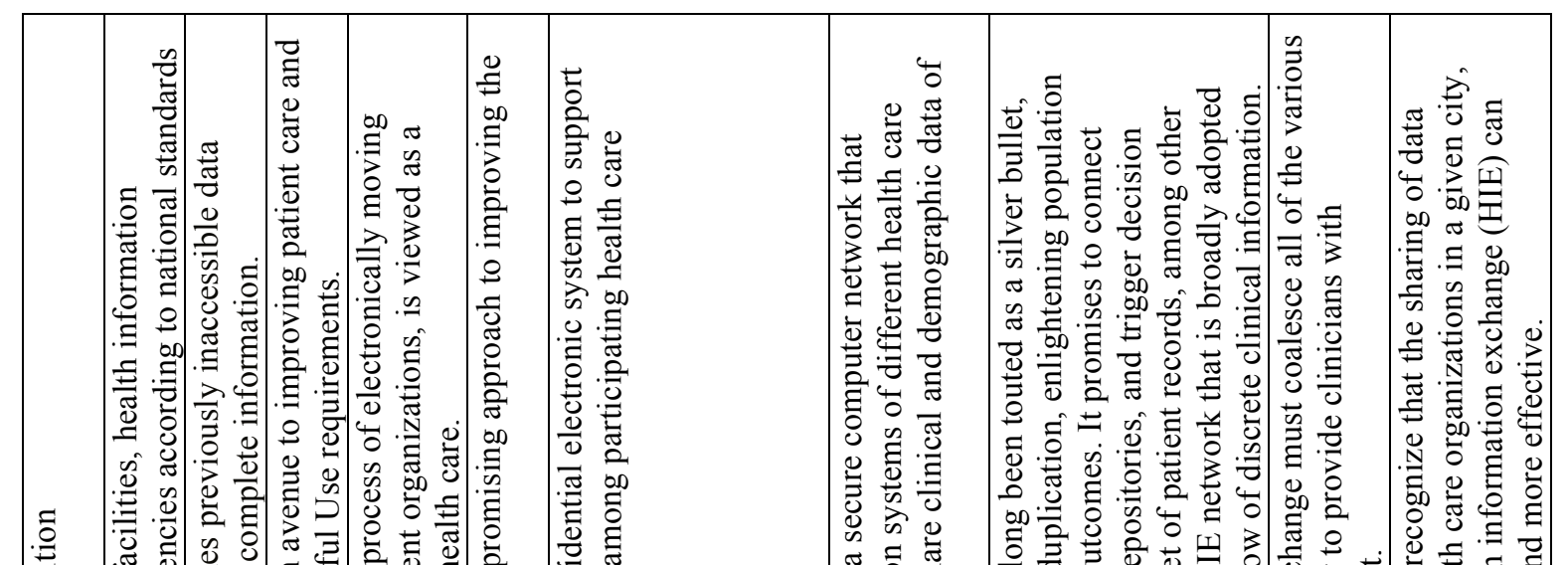

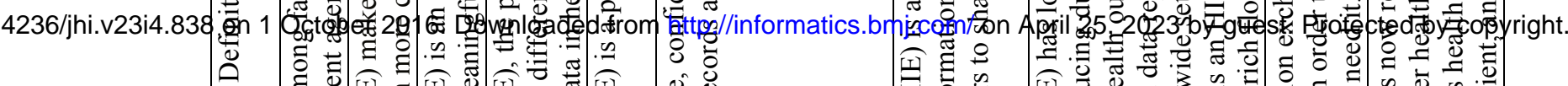

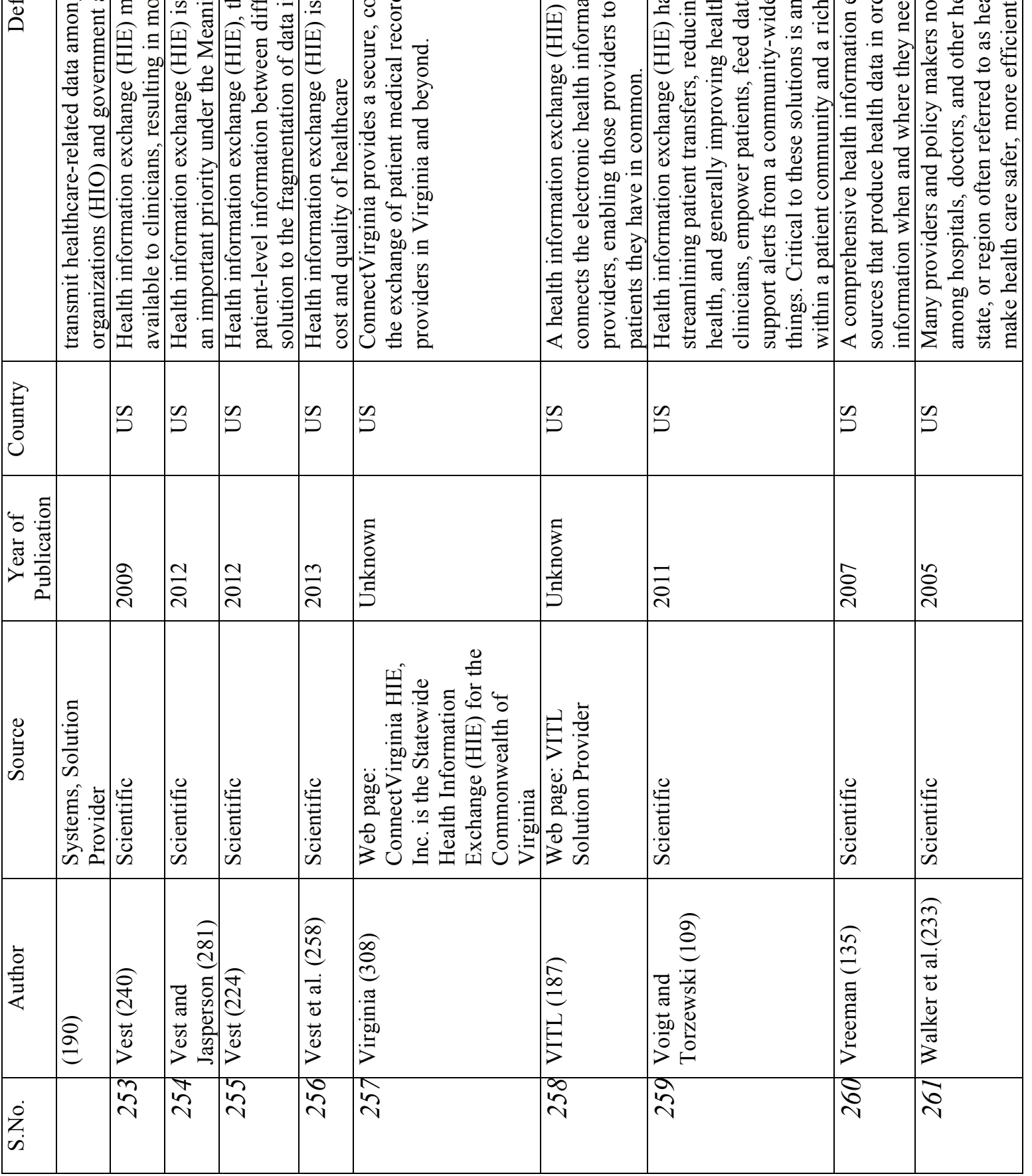


F

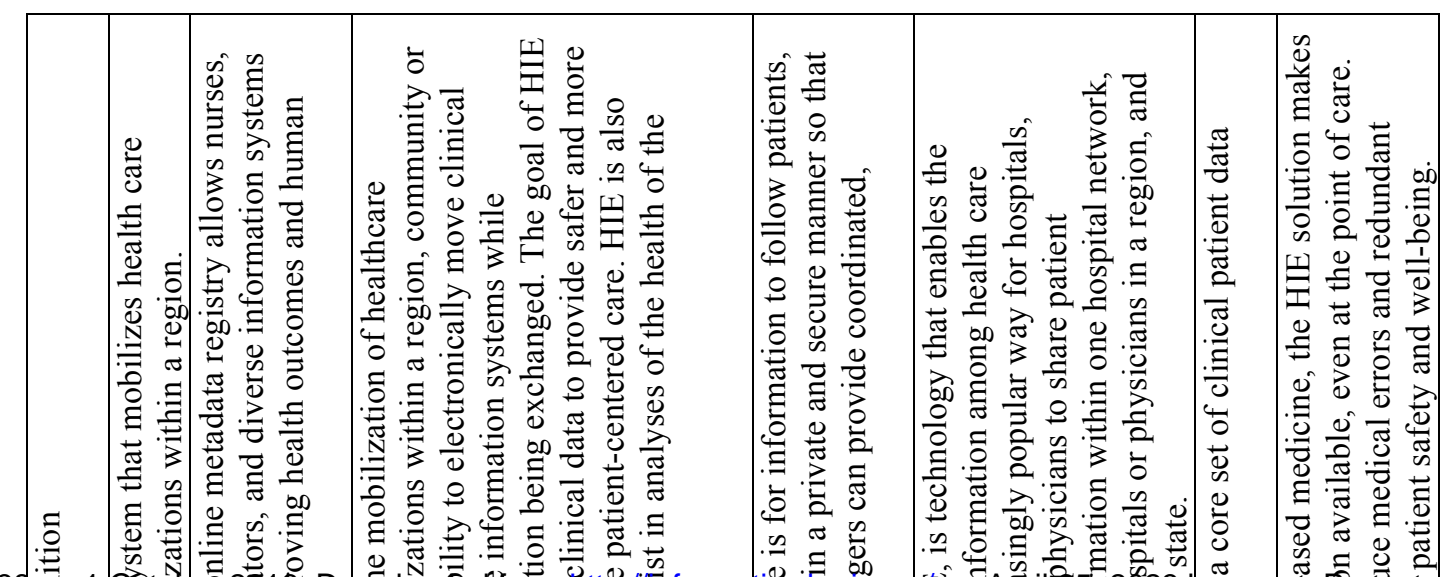

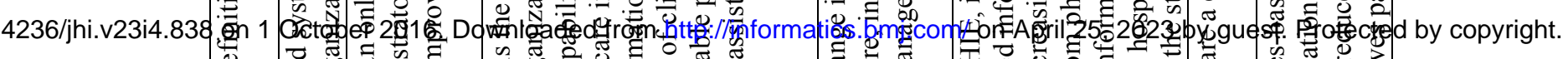

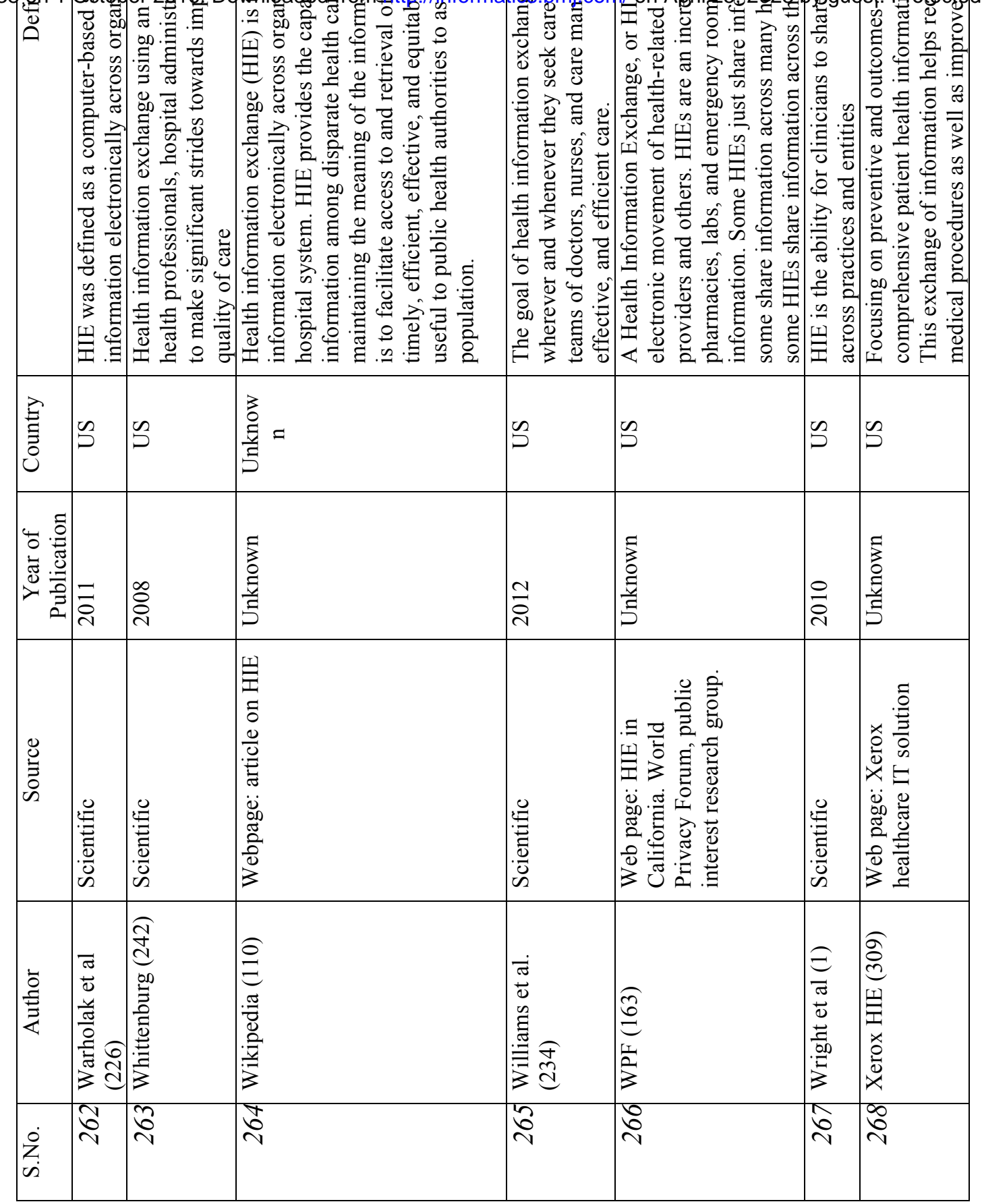


$\stackrel{+}{\sim}$

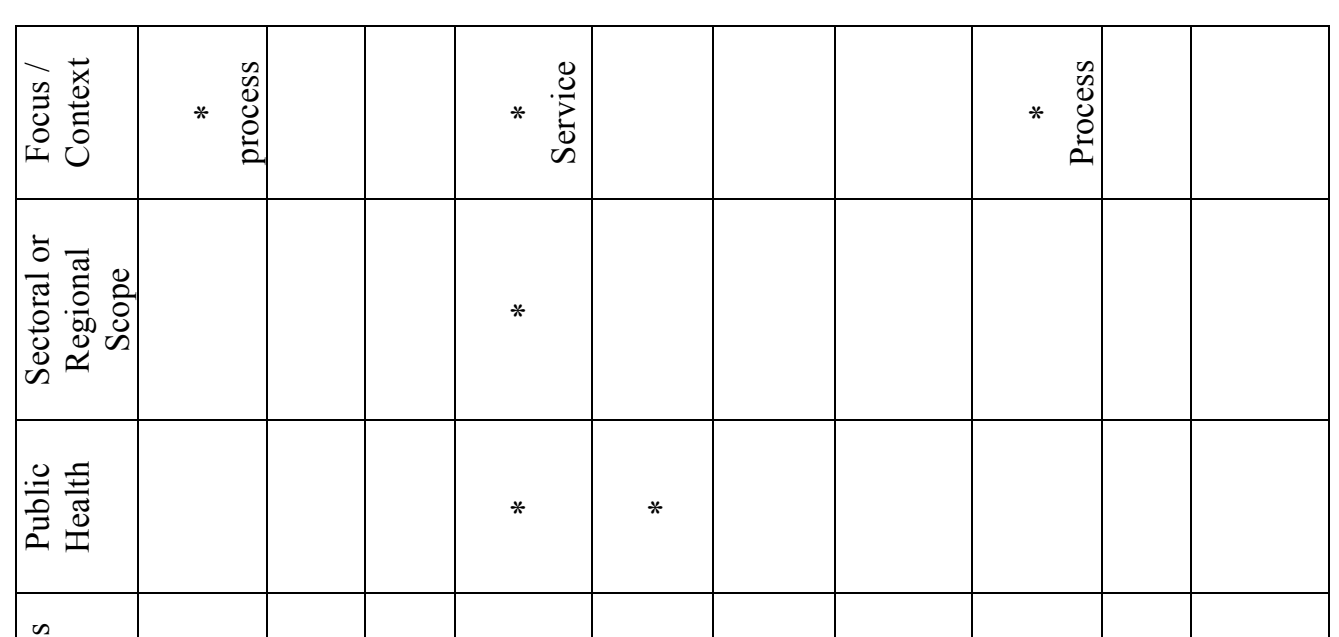

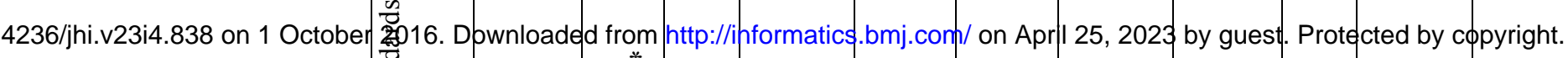

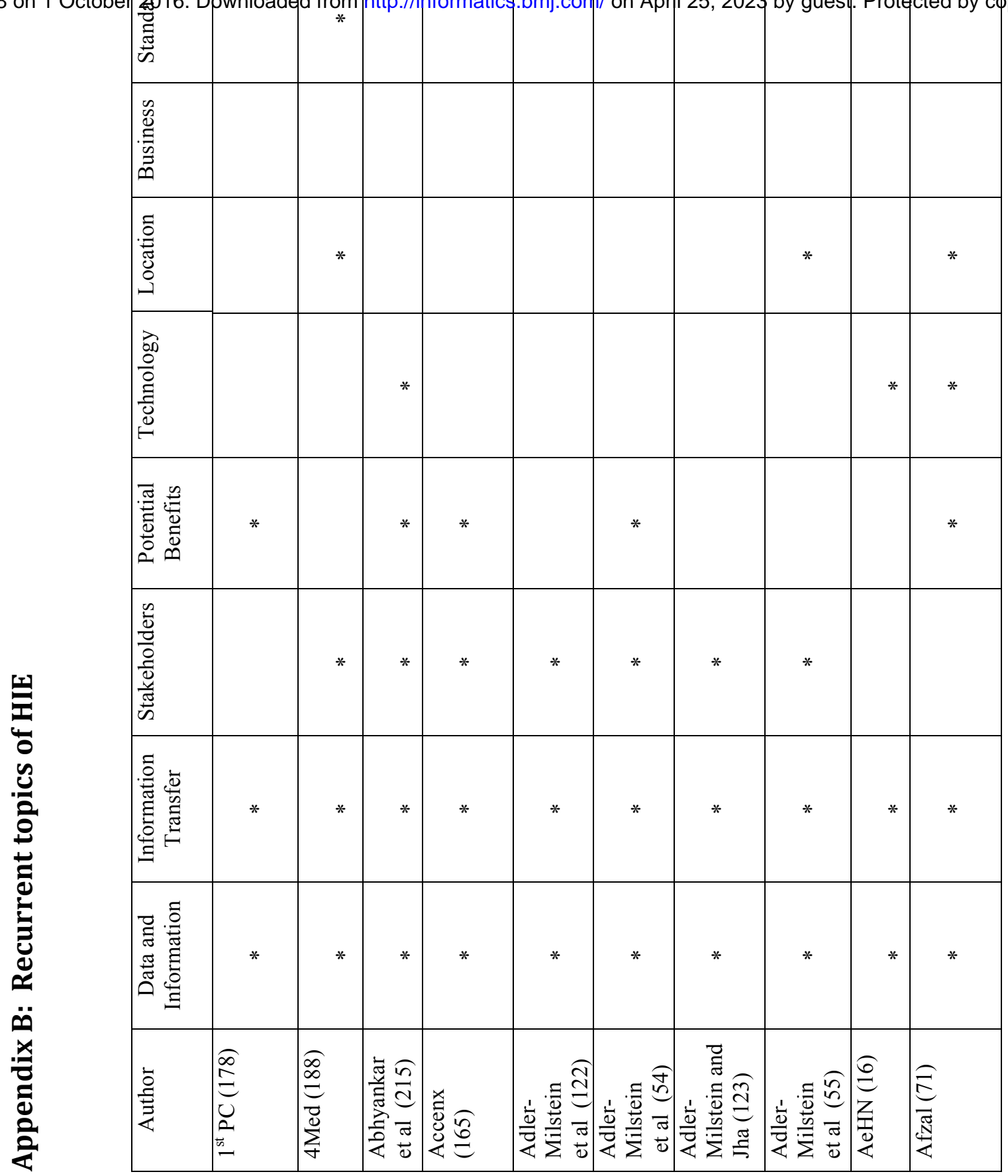



竎

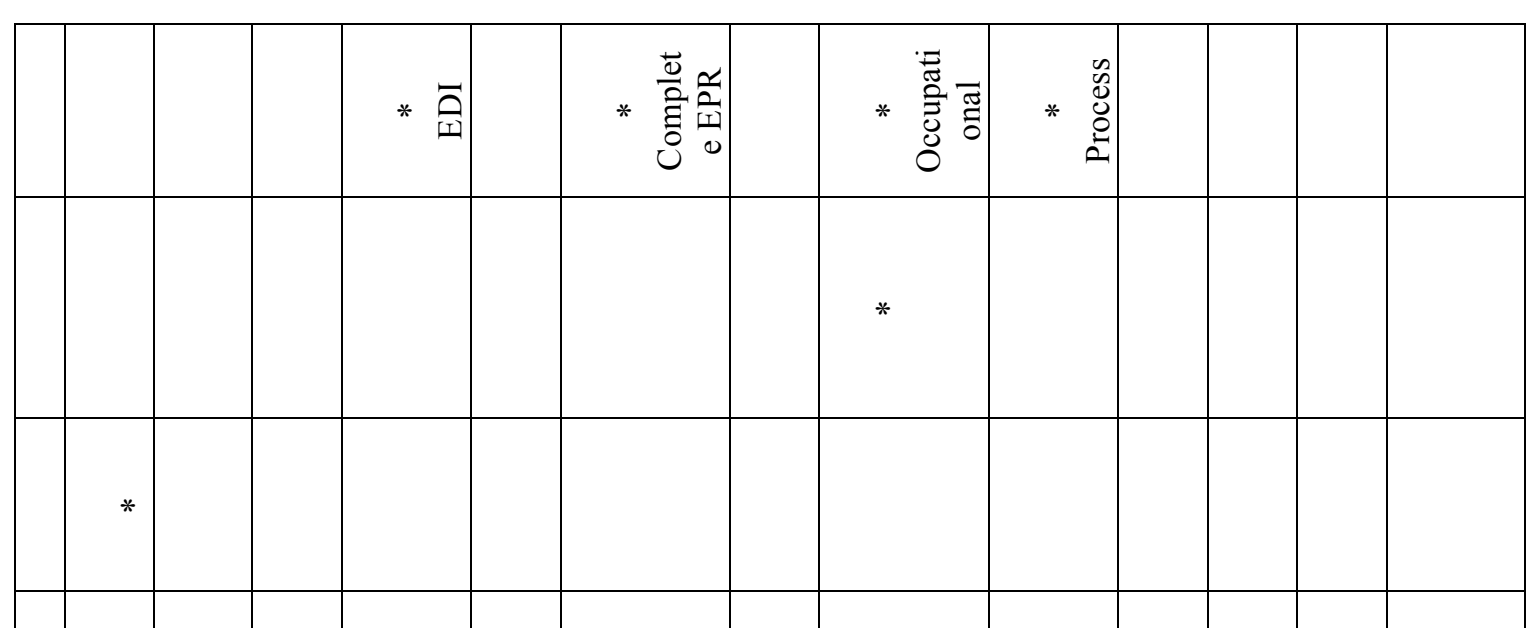

4236/jhi.v23i4.838 on 1 October 2016. Downloaded from http://informatics.bmj.com/ on April 25, 2023 by guest. Protected by copyright.

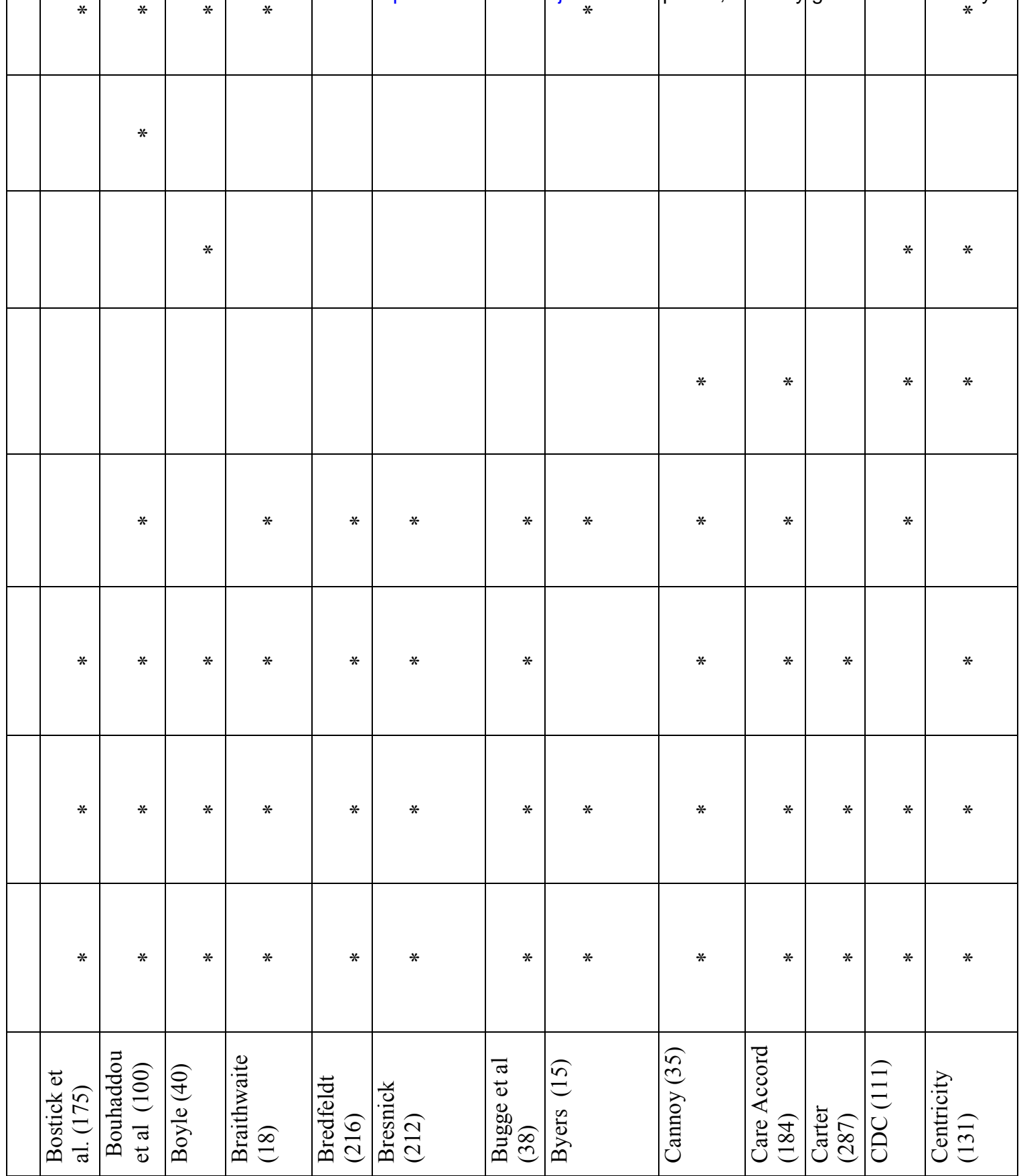


$\stackrel{\text { 舟 }}{2}$

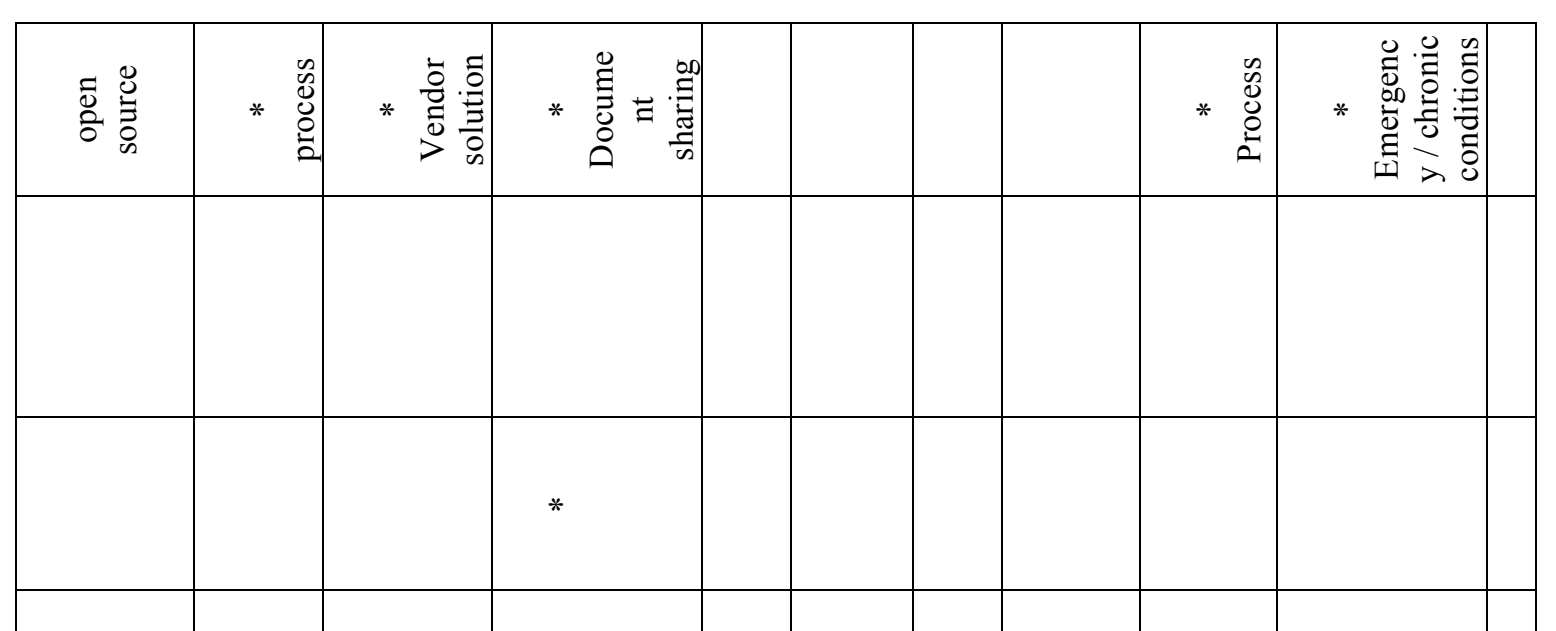

4236/jhi.v23i4.838 on 1 October 2016. Downloaded from http://informatics.bnj.com/ on April 25, 2023 by guest. Protected by copyright.

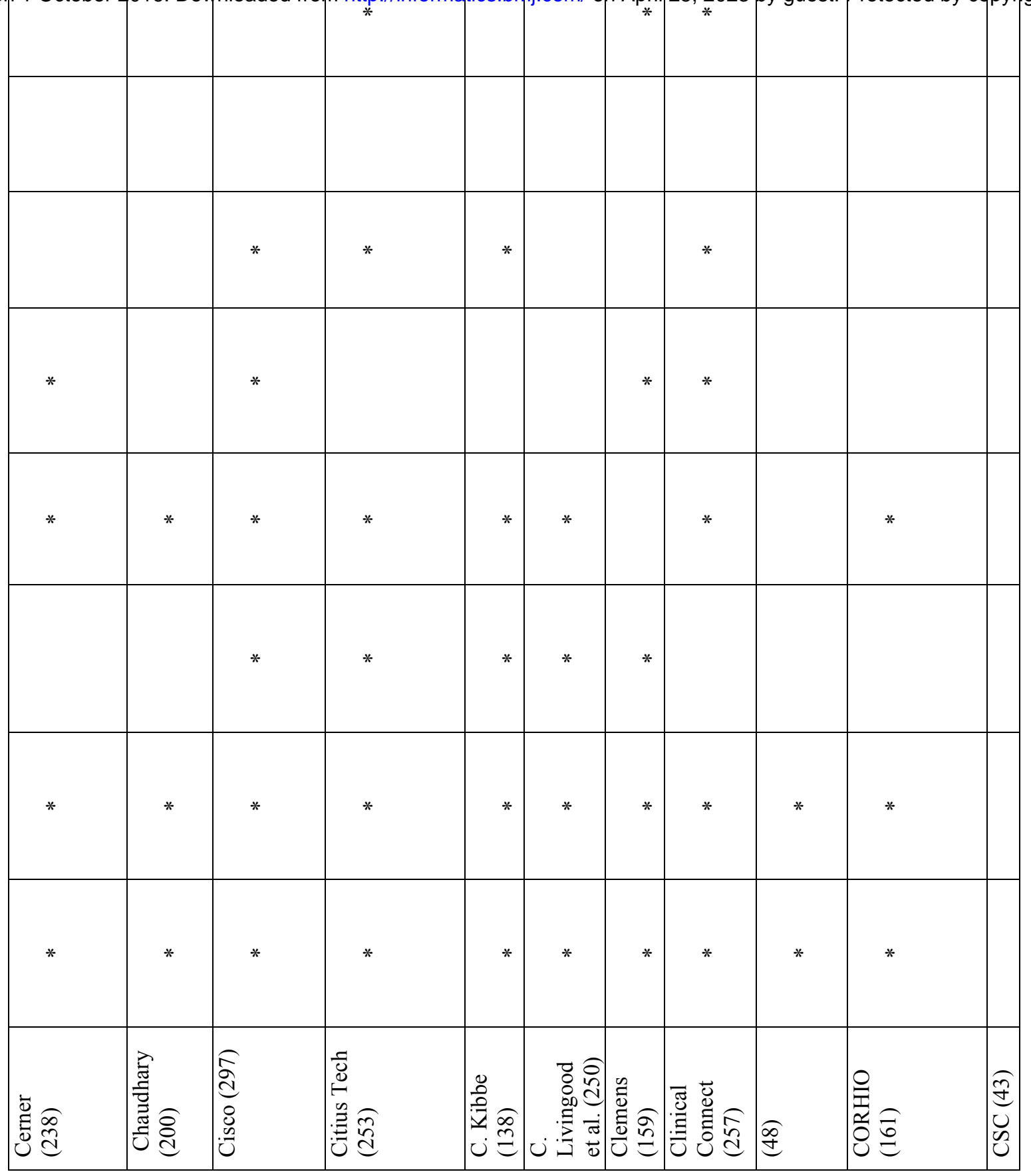


$\stackrel{0}{N}$

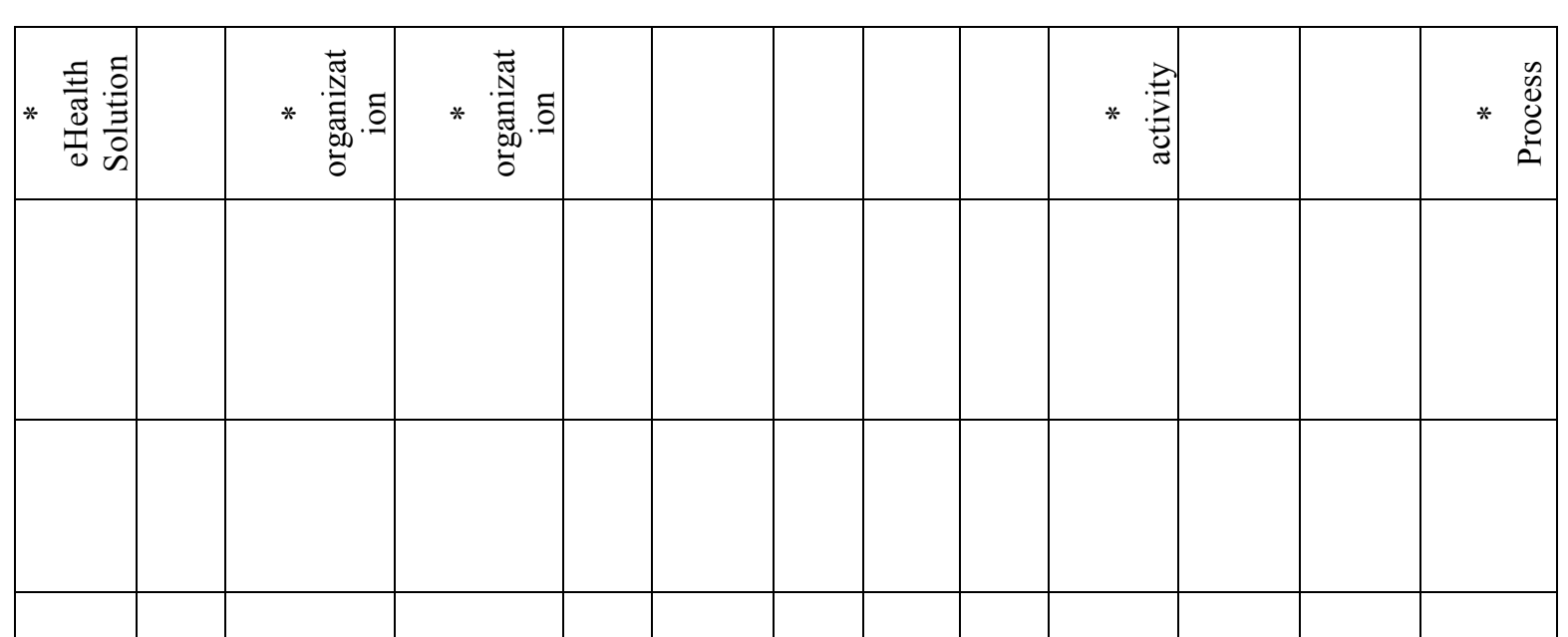

4236/jhi.v23i4.838 on 1 October 2016. Downloaded from http://informatics.bmj.com/ on April 25, 2023 by guest. Protected by copyright.

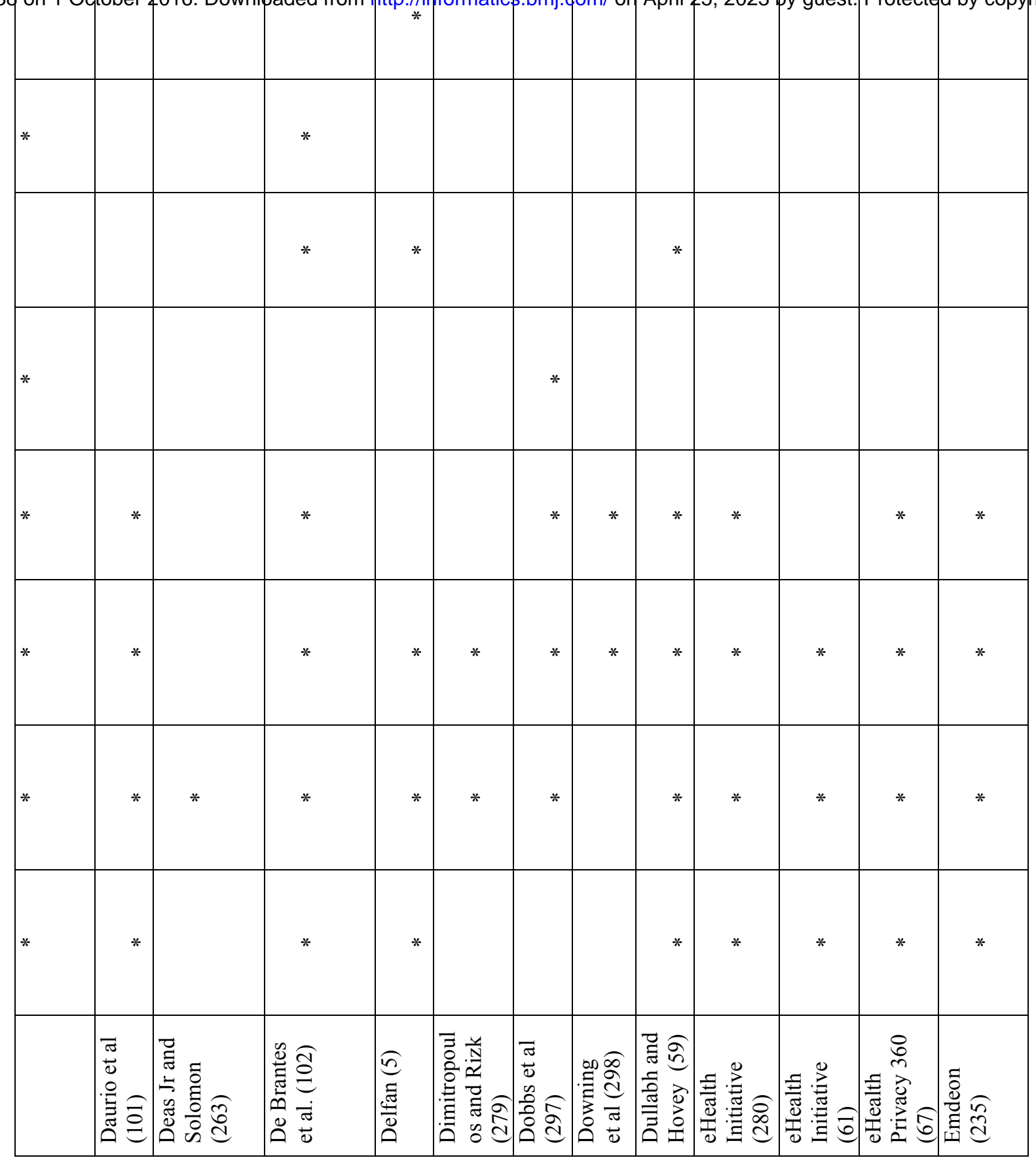


辛

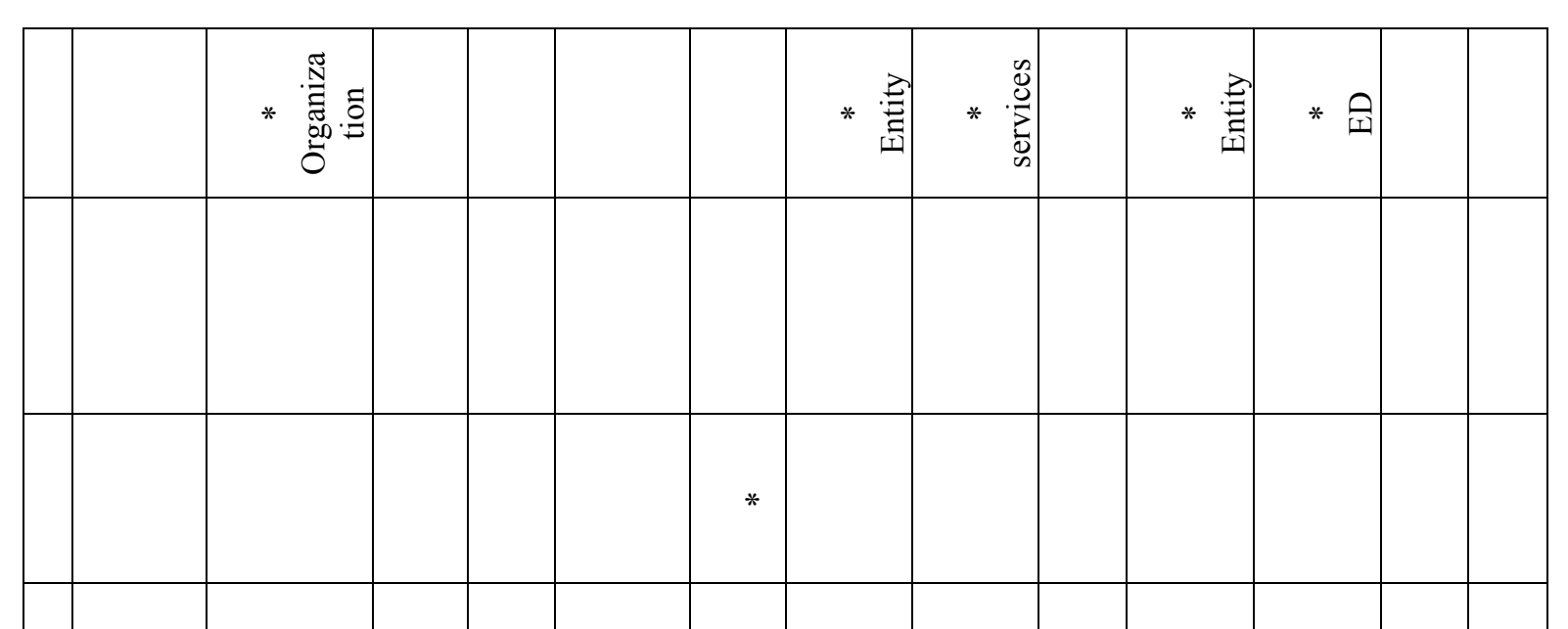

4236/jhi.v23i4.838 on 1 October 2016. Downloaded from http://informatics.bmj.com/ on April 25, 2023 by guest. Protected by copyright.

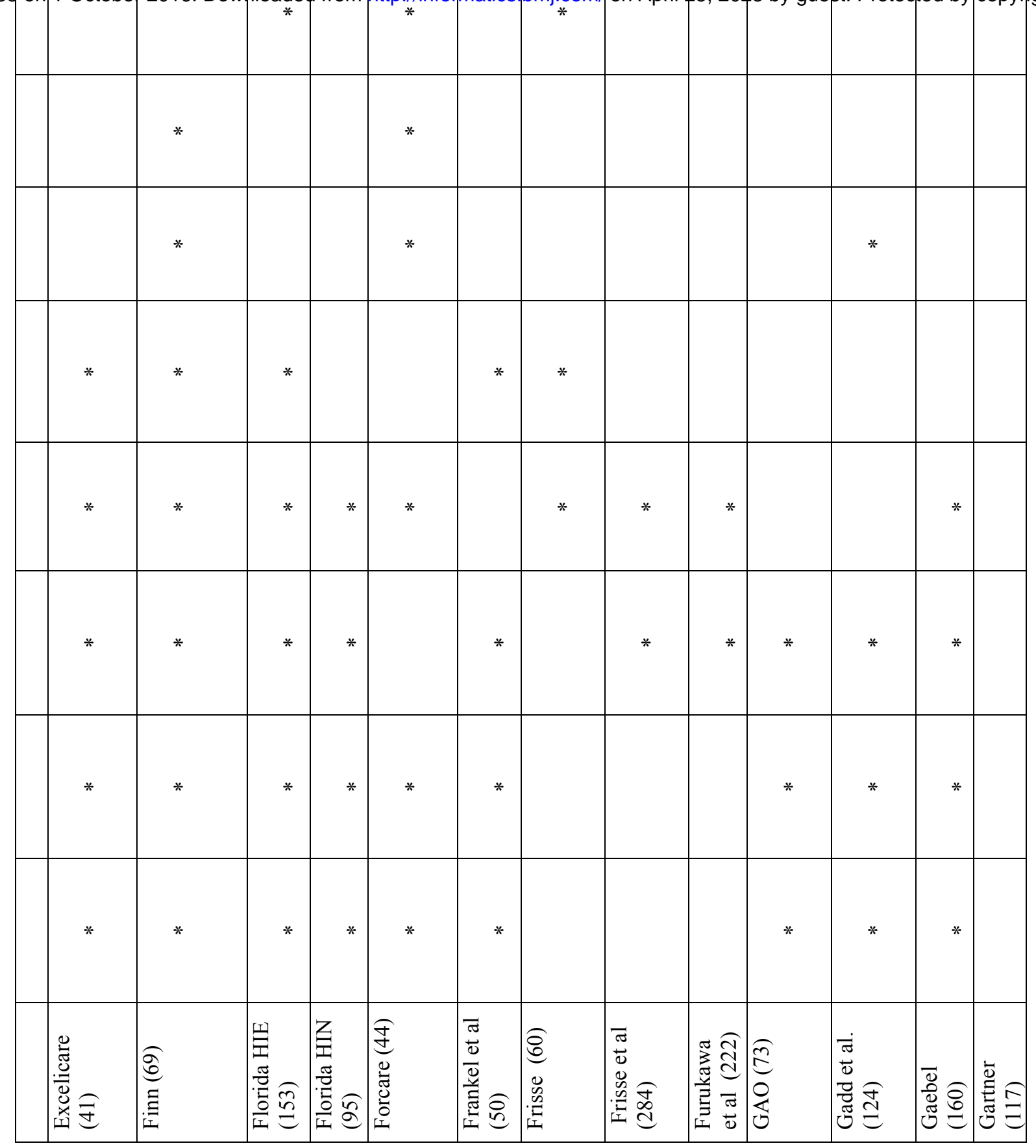


$\stackrel{\infty}{\wedge}$

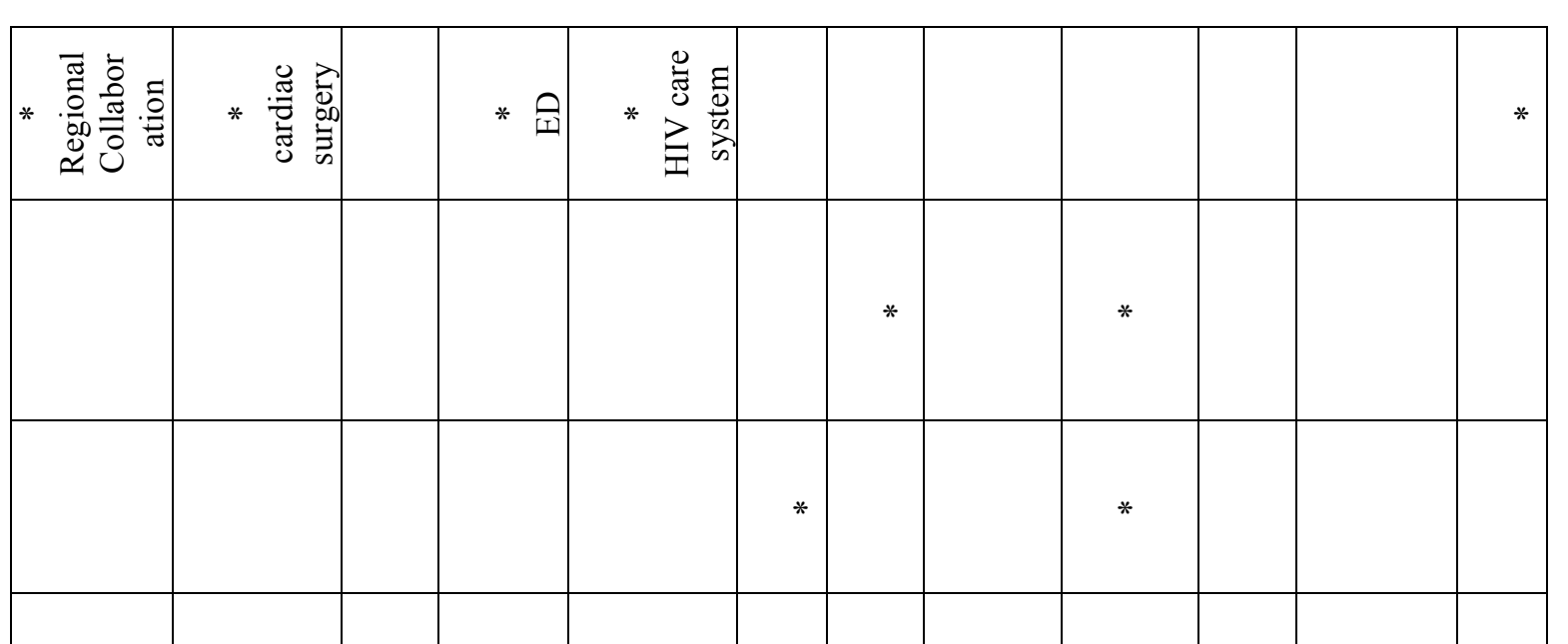

4236/jhi.v23i4.839 on 1 October 2016. Downloaded from http://informatics.bmj.com/ on April 25, 2023 by guest. Protected py copyright.

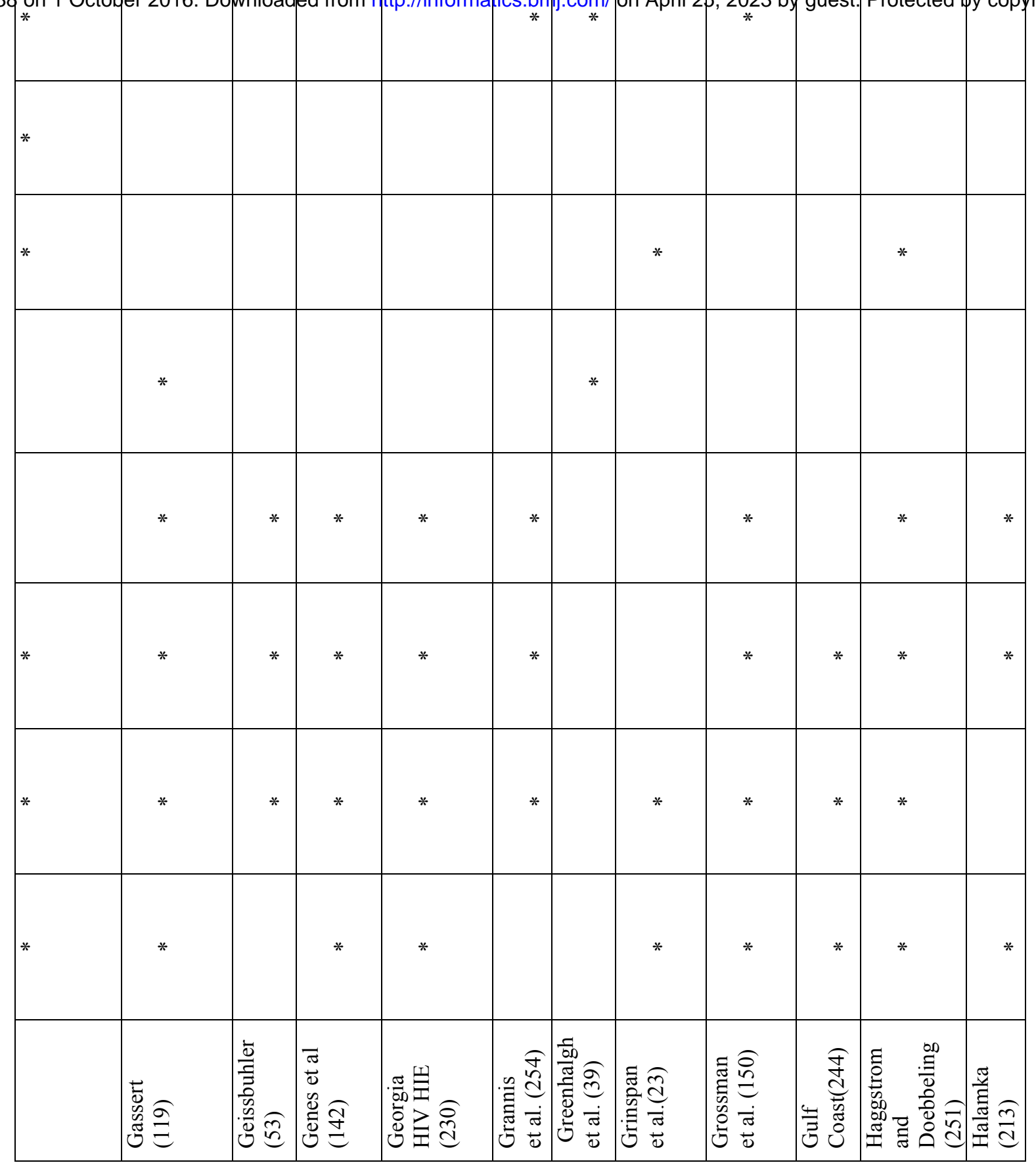


$\stackrel{g}{\wedge}$

4236/jhi.v23i4.838 on 1 Octфber 2016. Downloaded from http://informatics.bmj.com/ on April 25, 2023 by guest. Protected by copyright.

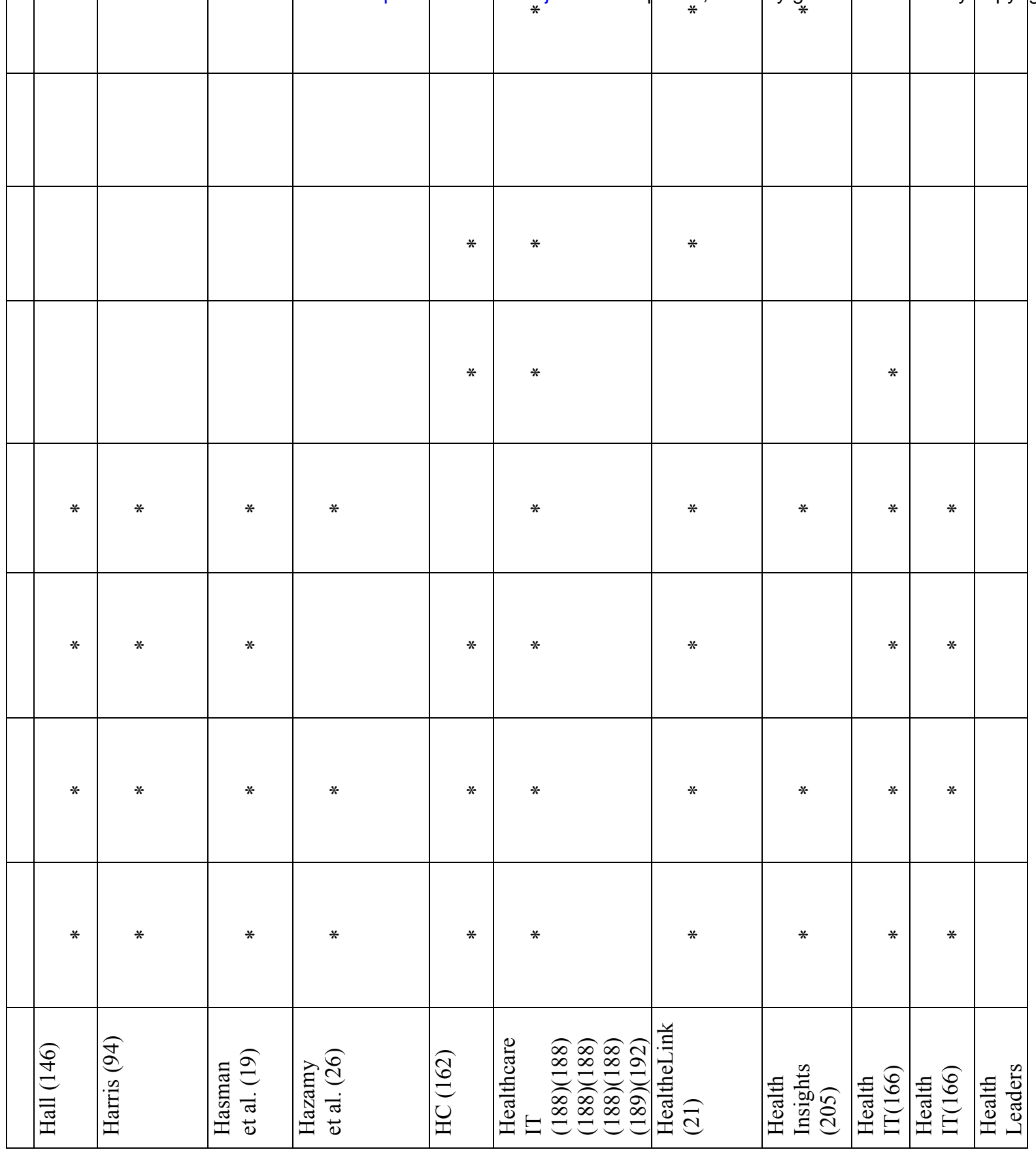


员

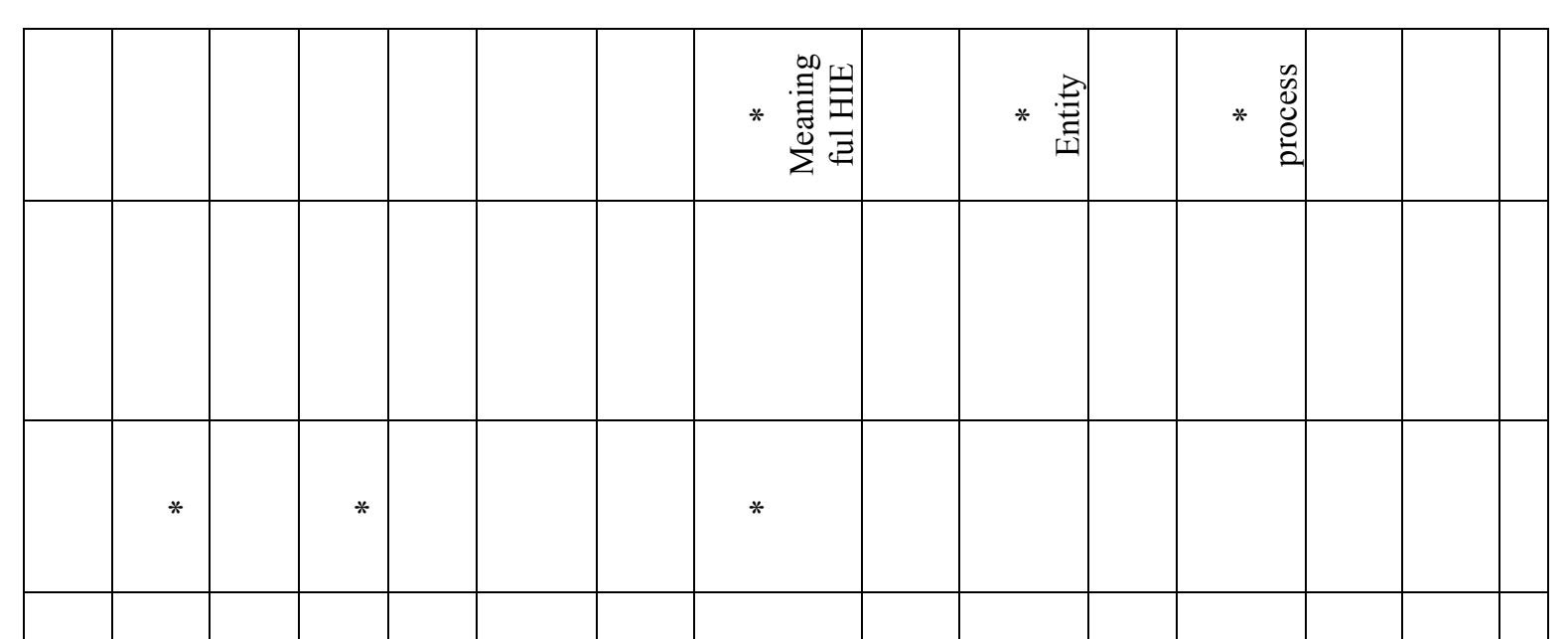

4236/jhi.v23i4.838 on 1 Dctober 2016. Downloaded from http://informatics.bmj.com/ on April 25, 2023 by guest. Protected by copyright.

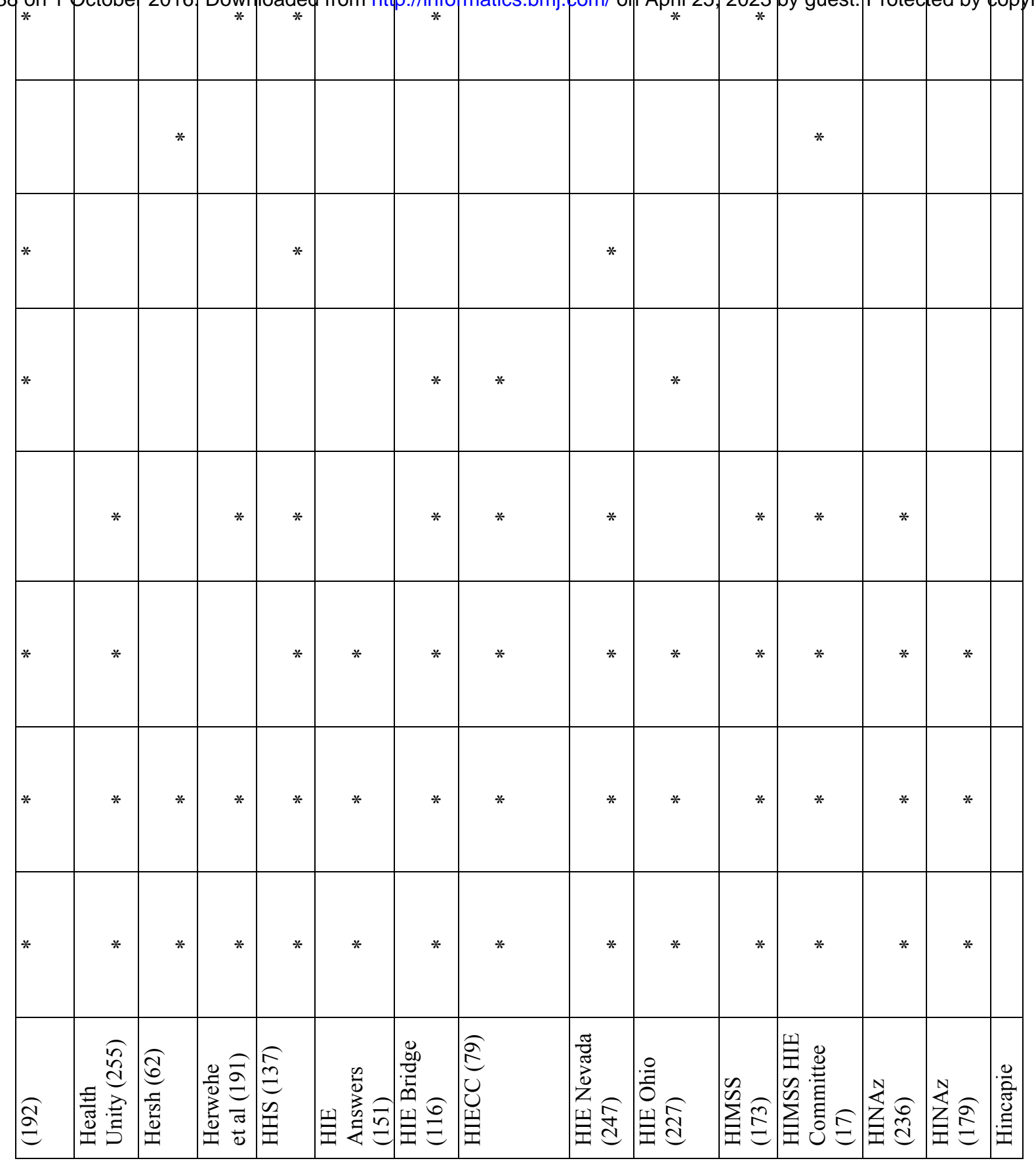


롯

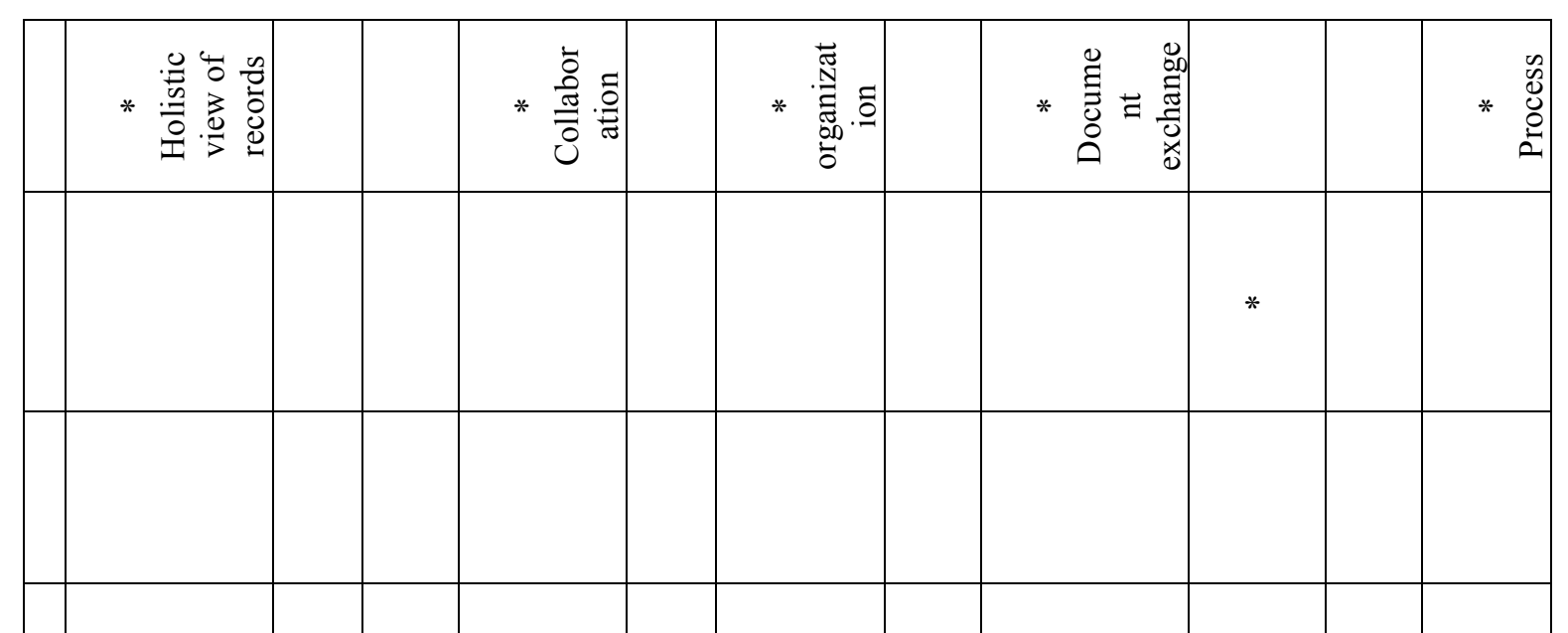

4236/jhi.v23i4.838 on 1 October 2016. Downloaded from http://informatics.bmj.com/ on April 25, 2023 by guest. Protected by copyright.

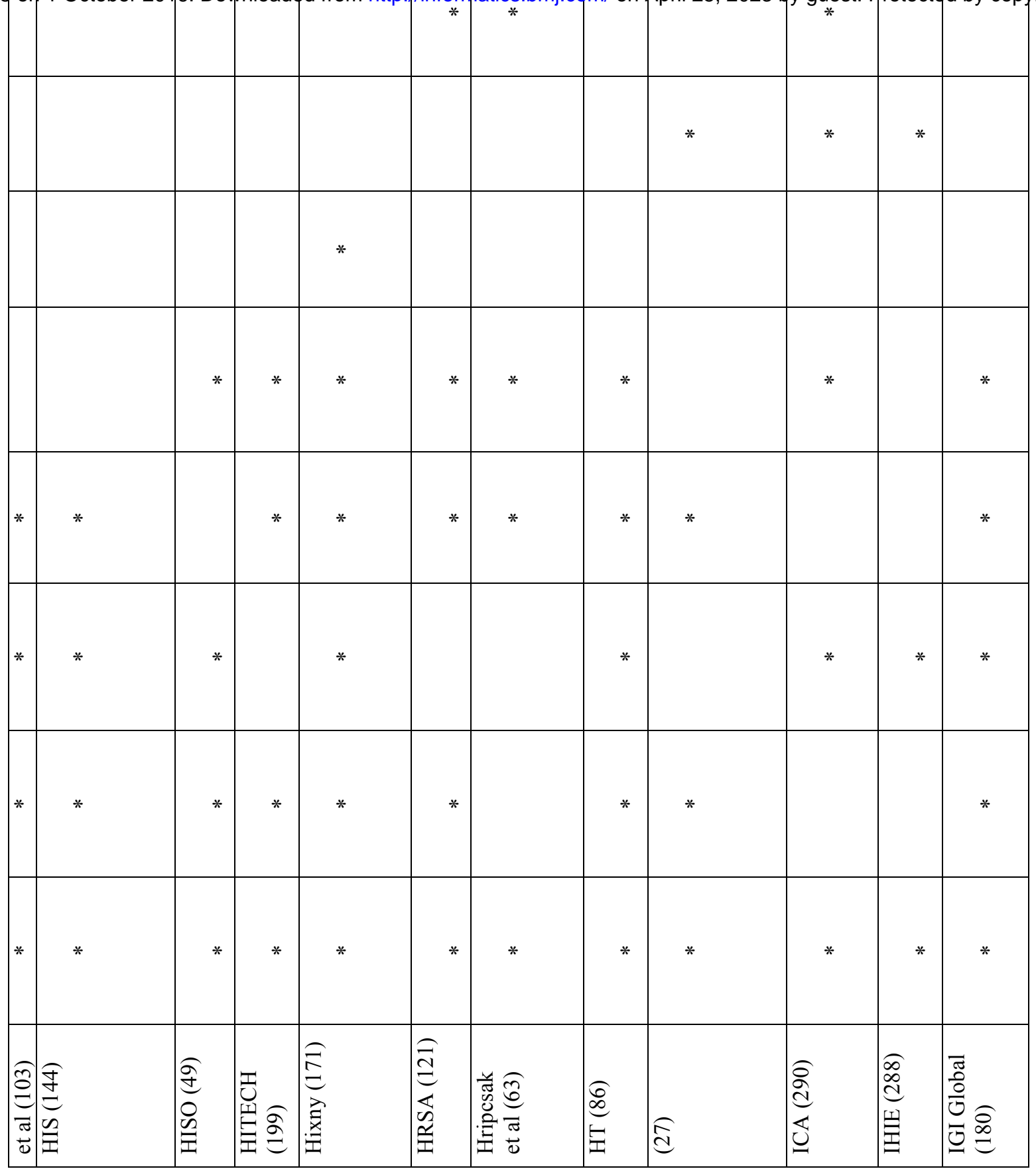


กิ

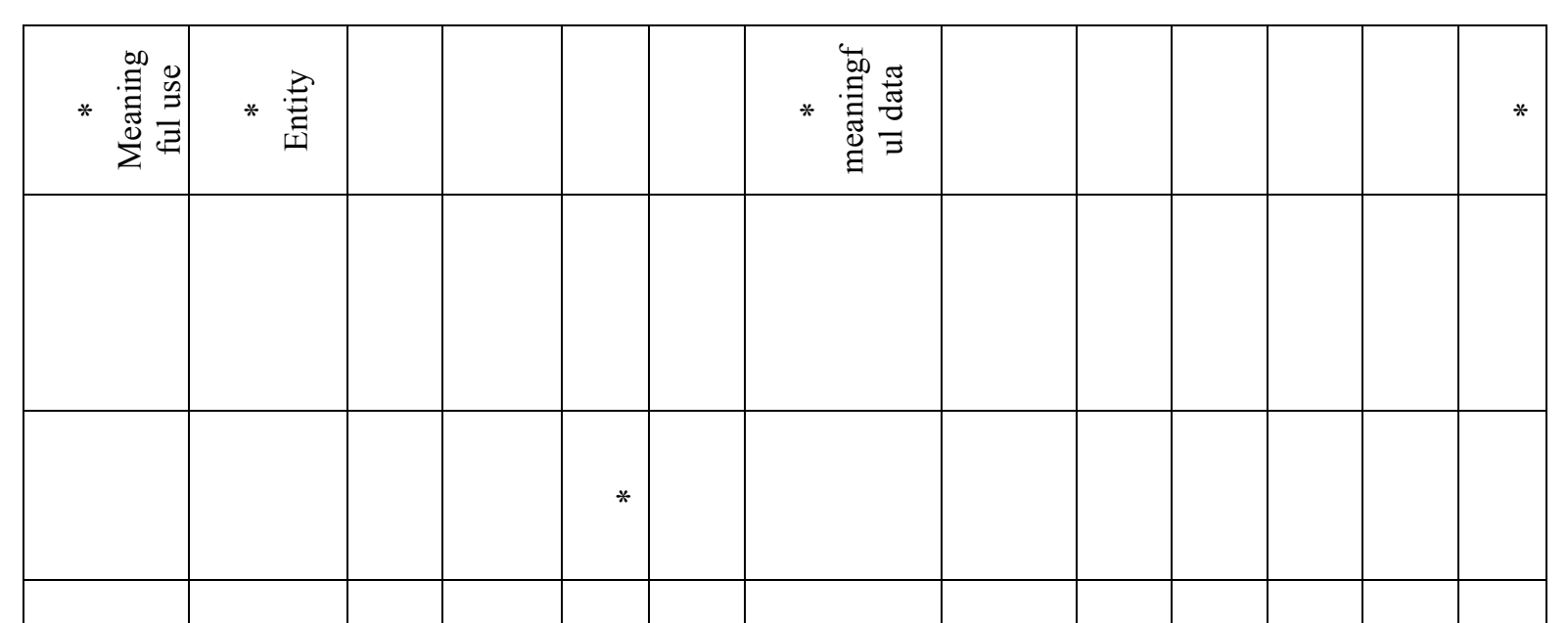

4236/jhi.v23i4.83 on 1 October 2016. Downloaded from http://informat/cs.bmj.com/ on April 25, 2023 by guest. Protected by copyright.

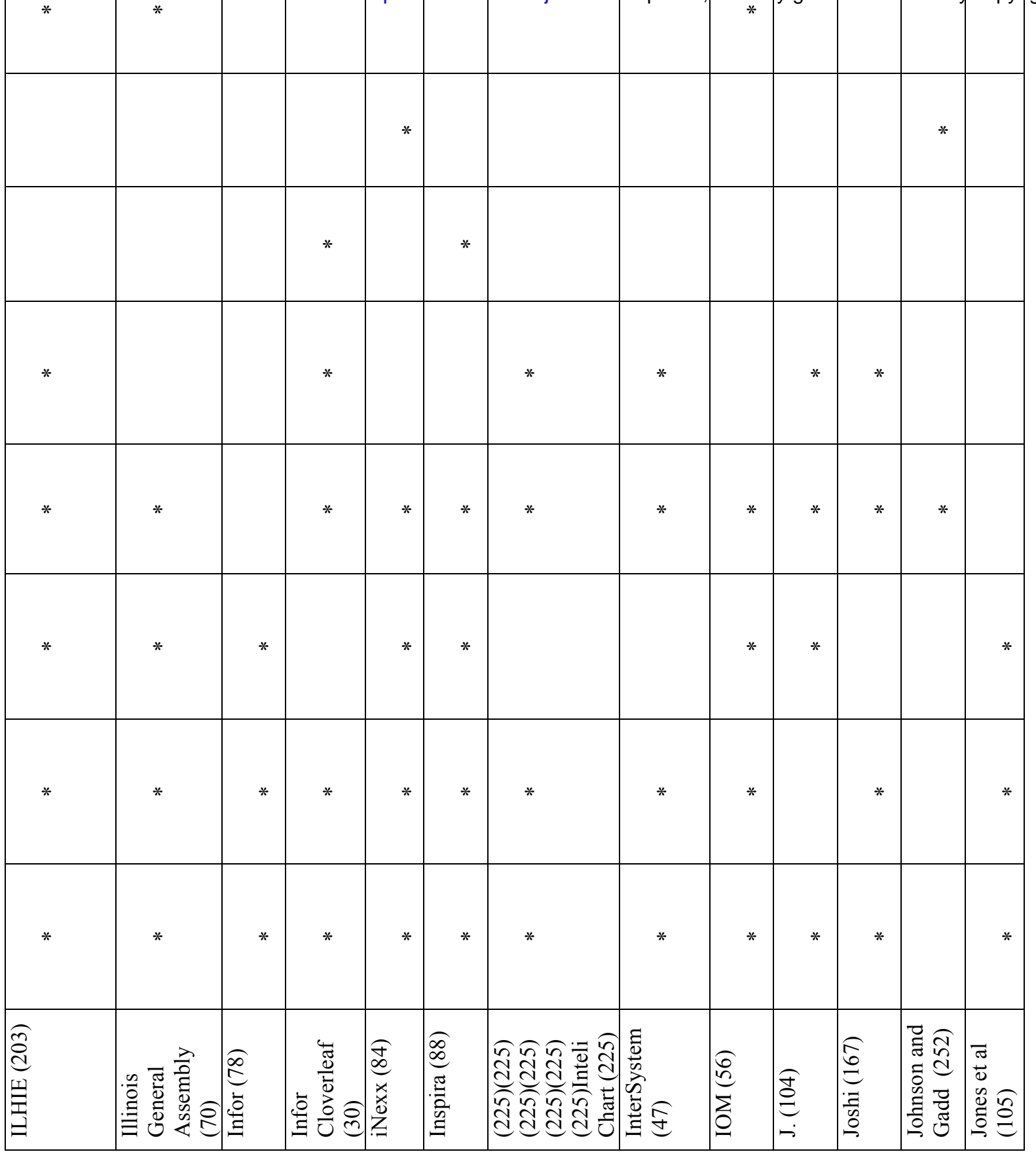


$\stackrel{n}{N}$

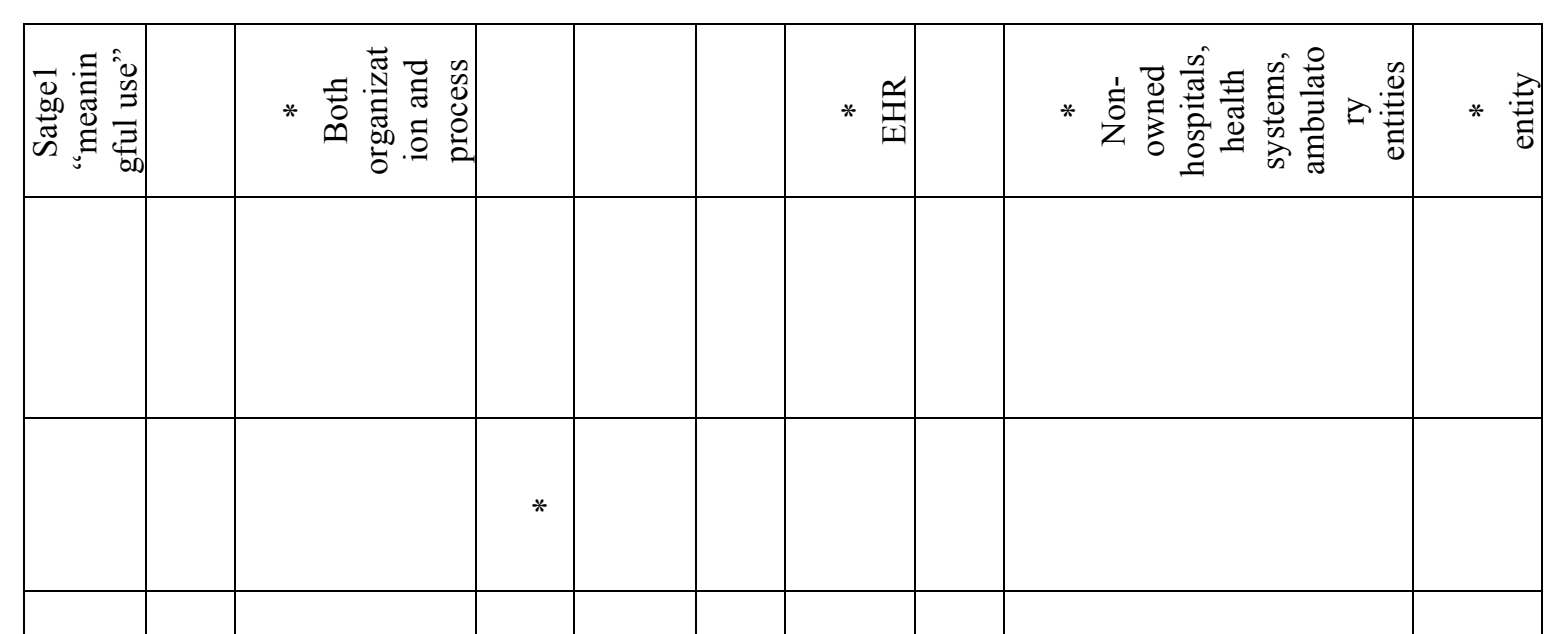

4236/jhi.v23i4.838 on 1 October 2016. Downloaded from http://informatics.bmj.com/ on April 25, 2023 by guest. Protected by copyright.

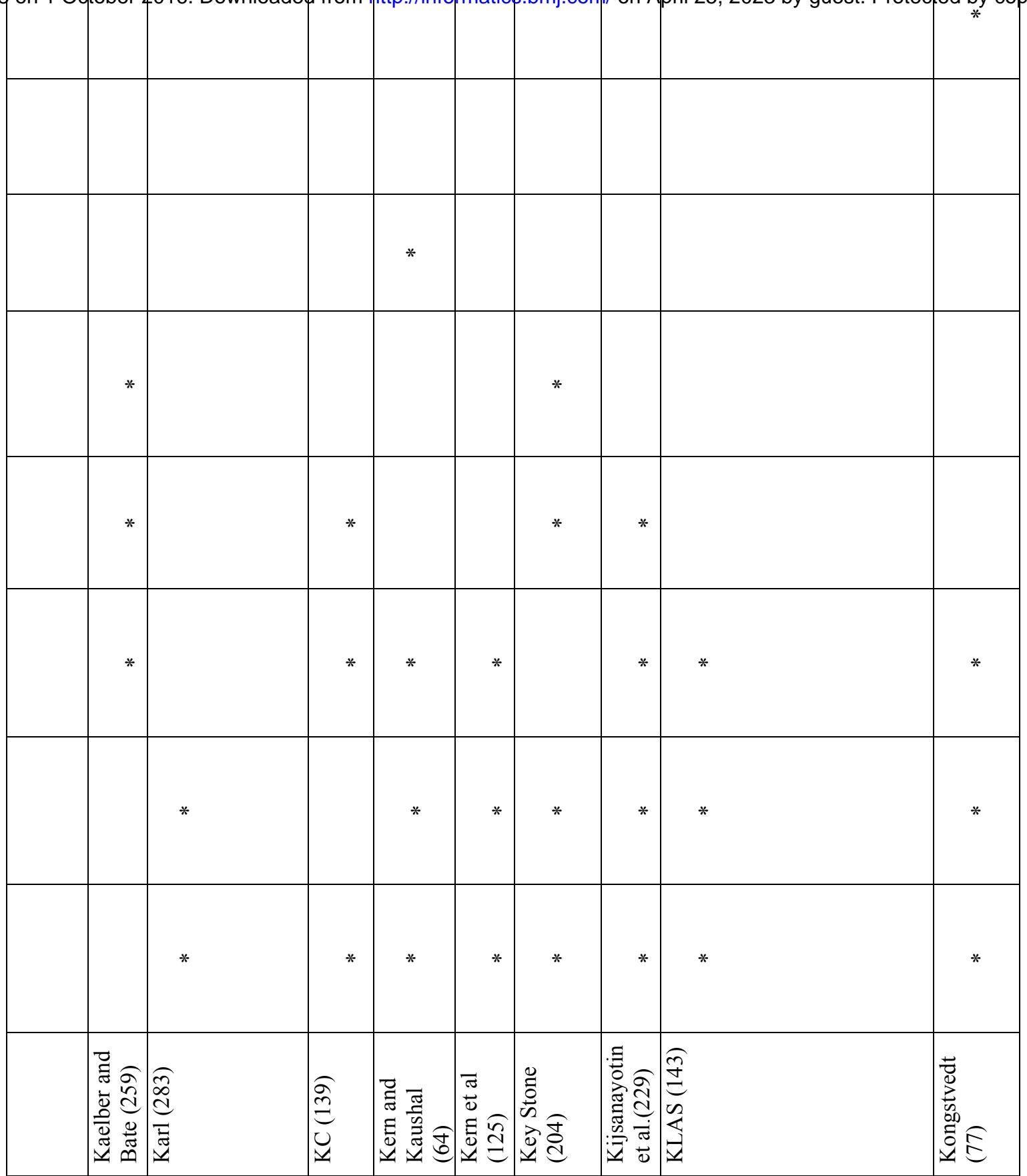




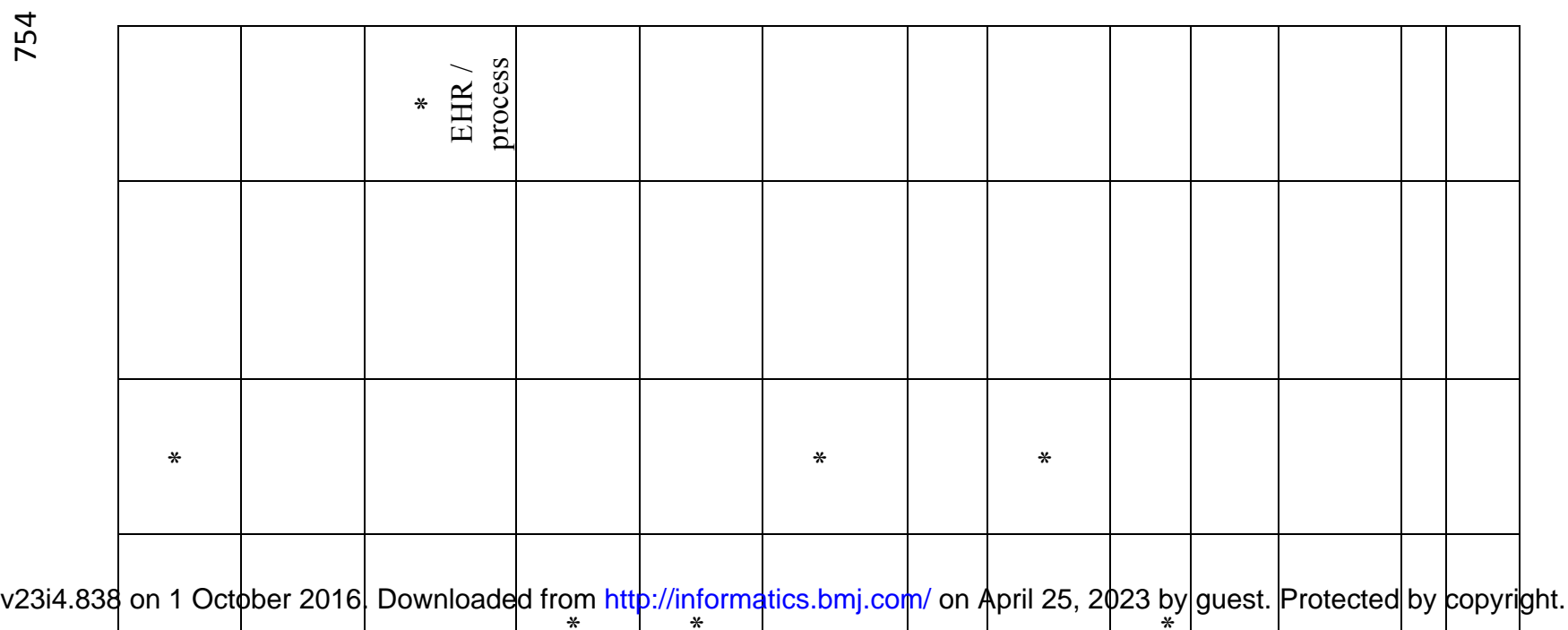

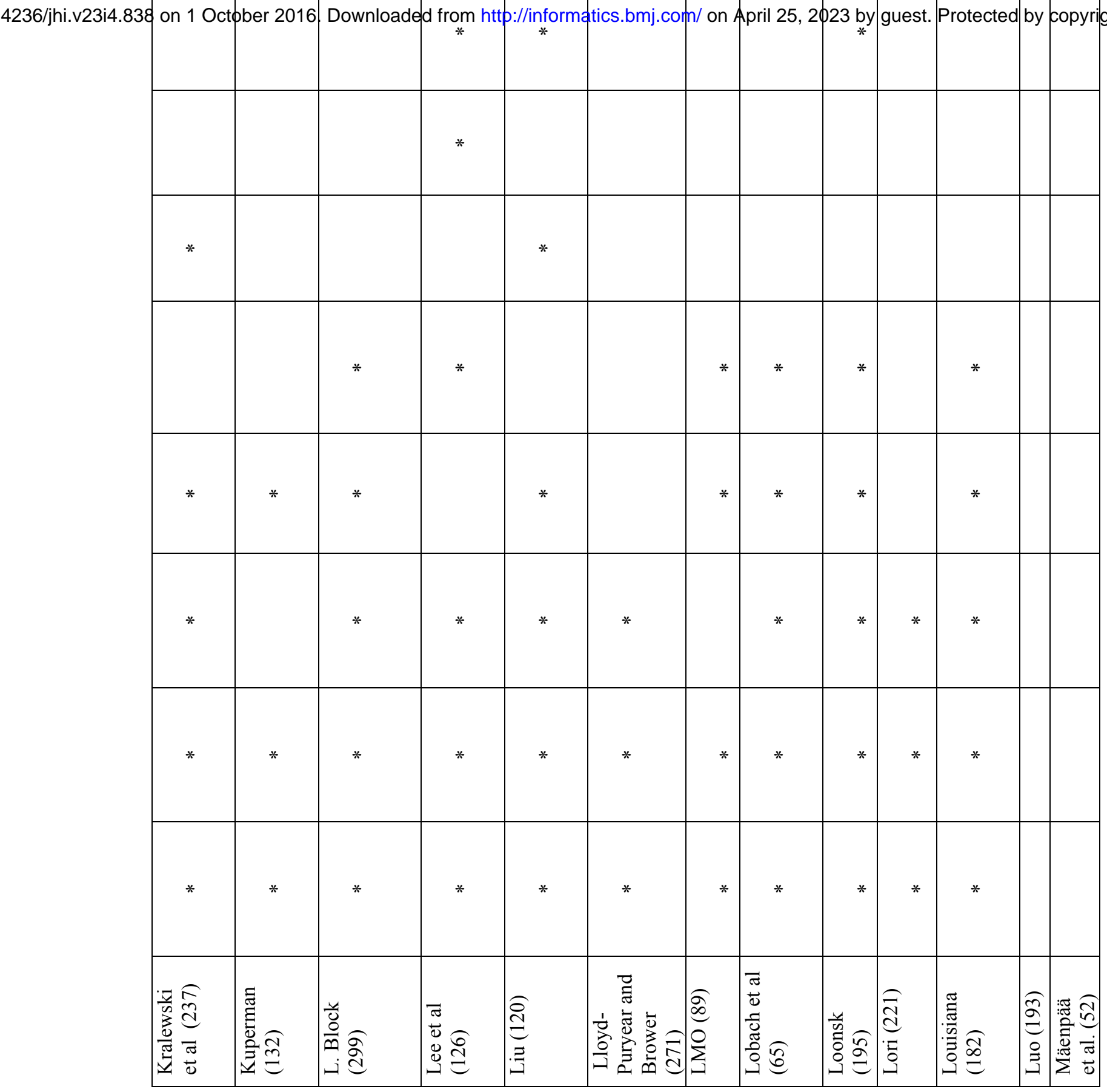


员

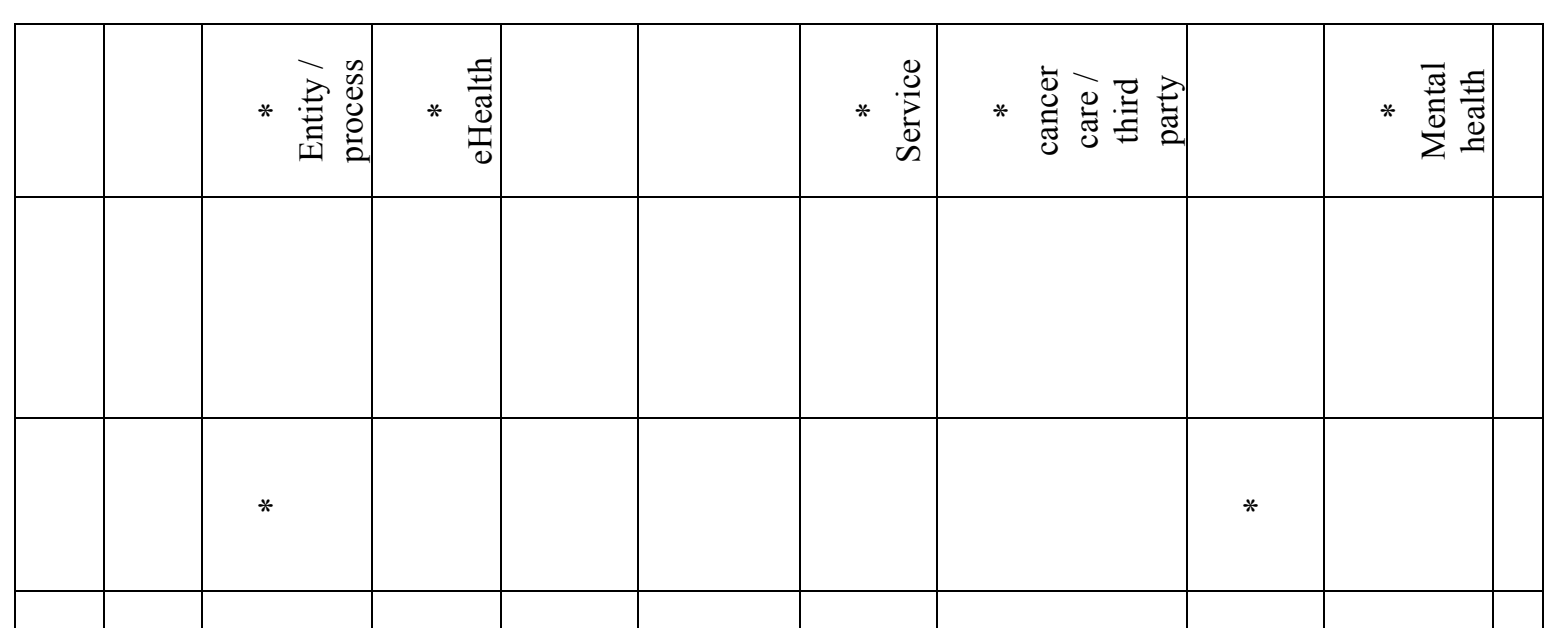

4236/jhi.v23i4.838 on 1 October 2016. Downloaded from http://nformatics.bmj.com/ on April 25, 2023 by guest. Protected by copyright.

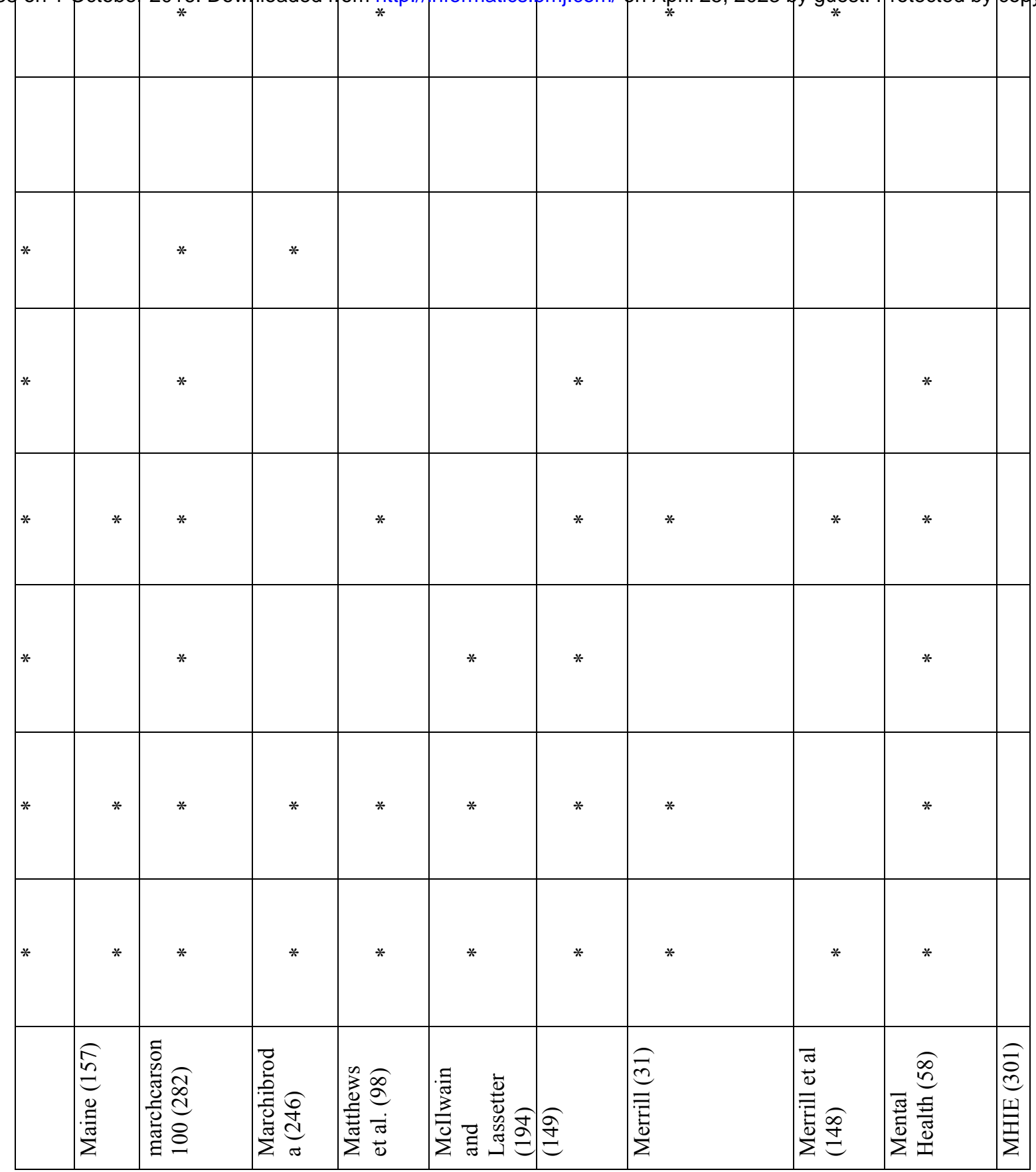



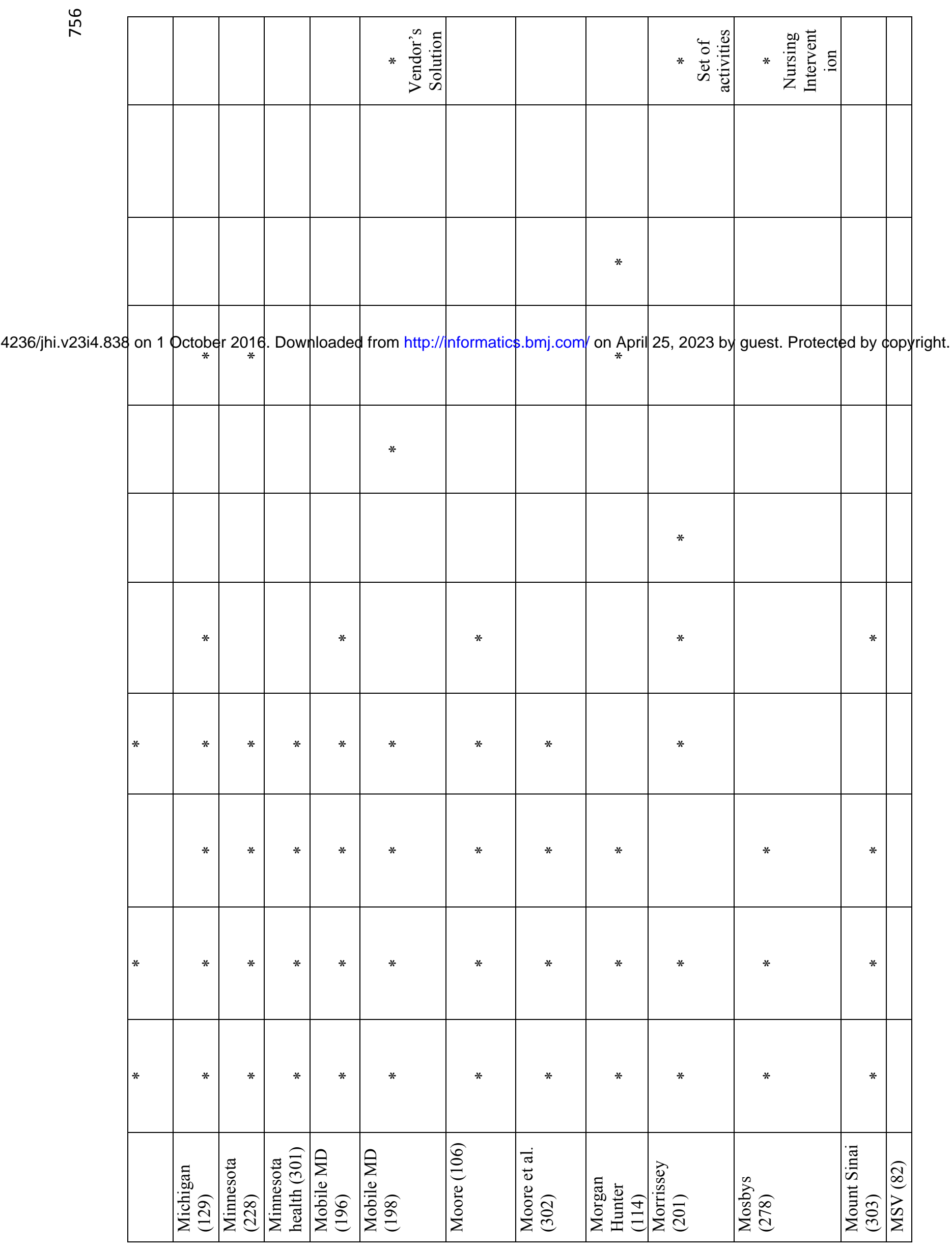


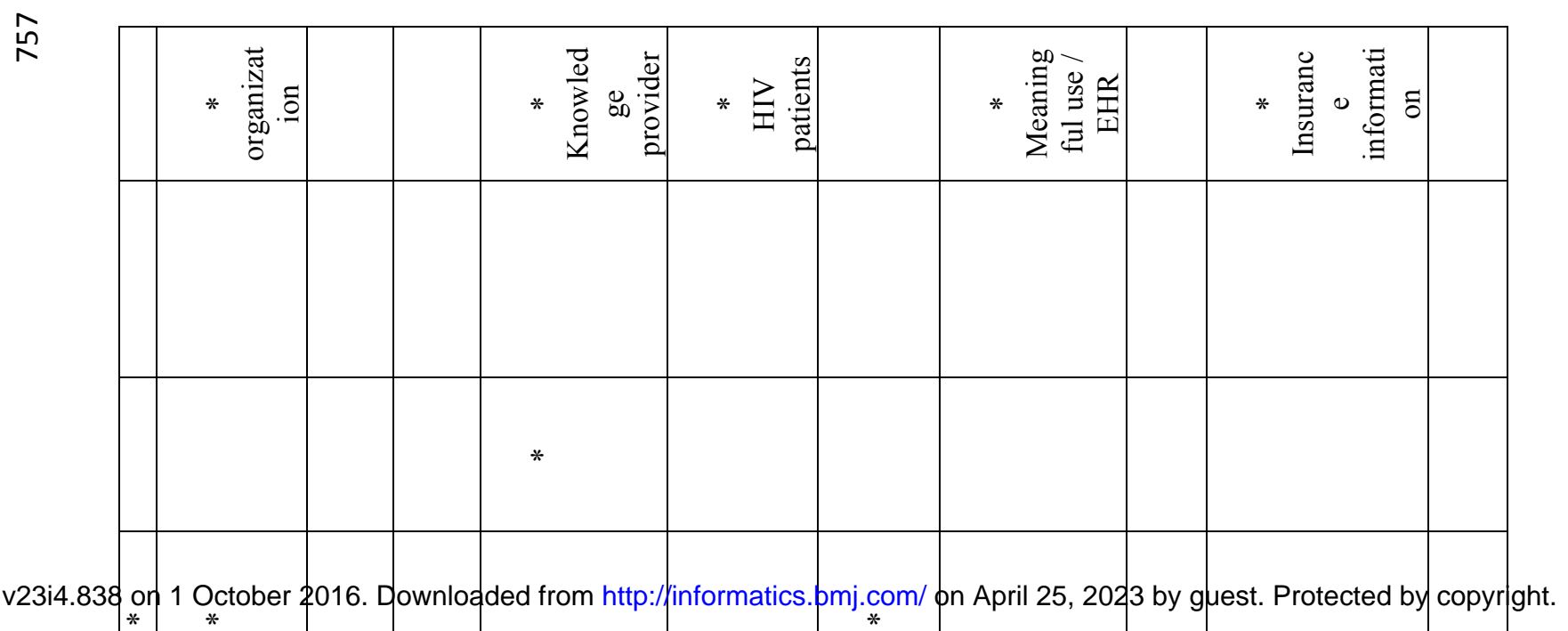

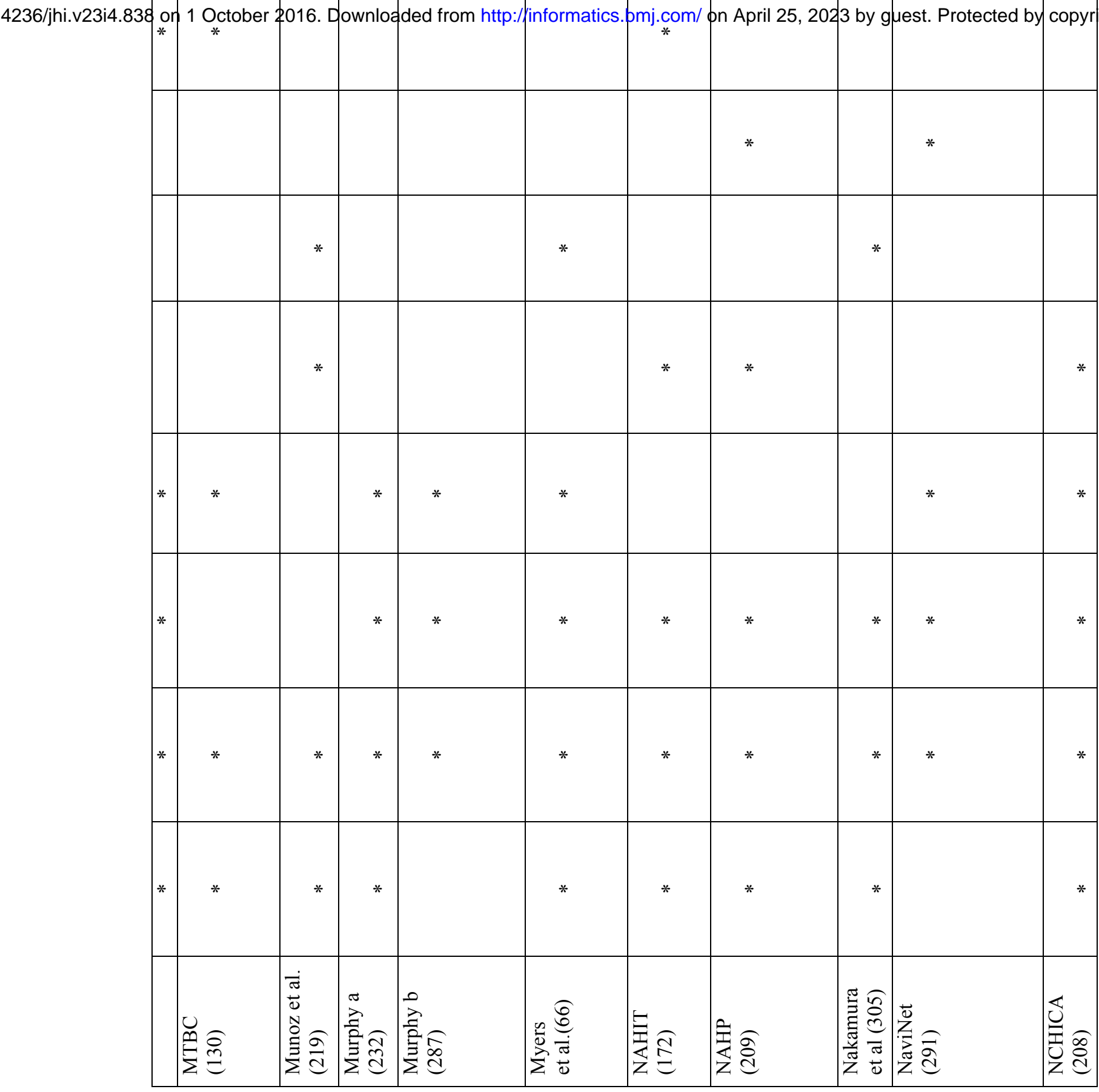


$\stackrel{\infty}{\stackrel{\infty}{N}}$

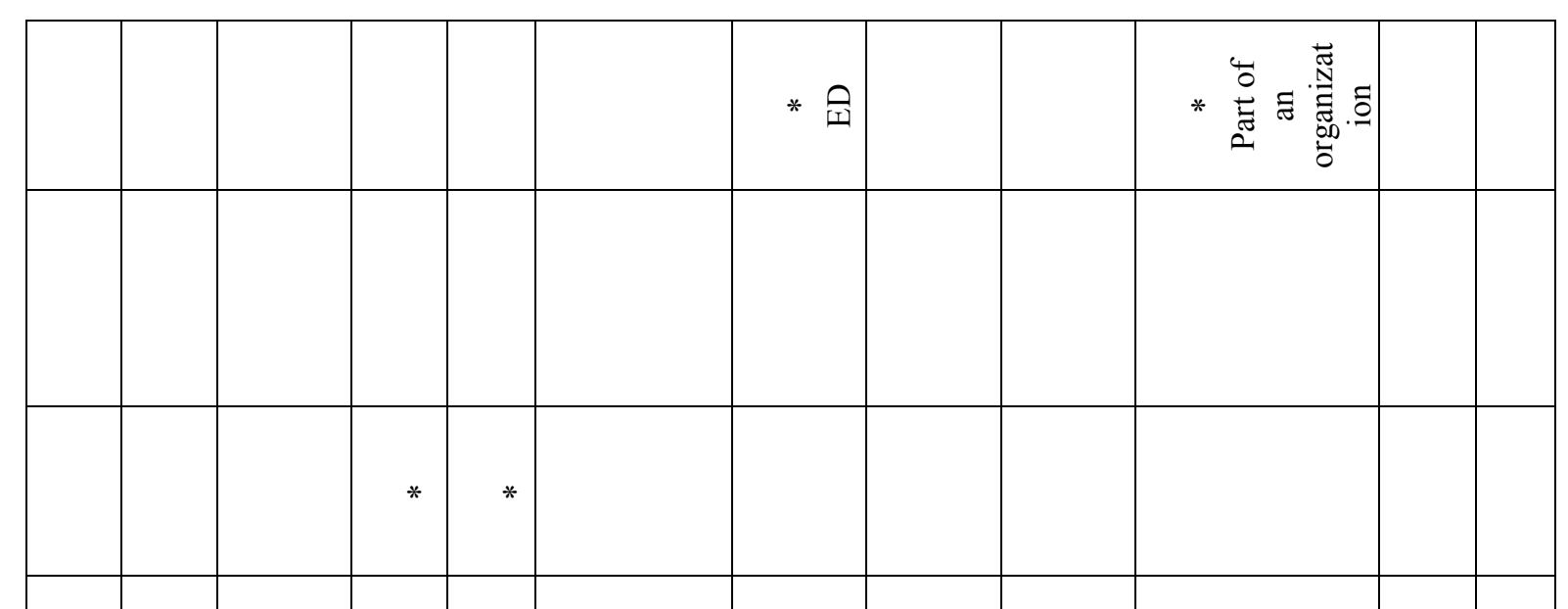

4236/jhi.v23i4.838 on 1 October 2016. Downloaded from http://informatics.bmj.com/ on April 25, 2023 by guest. Protected by copyright.

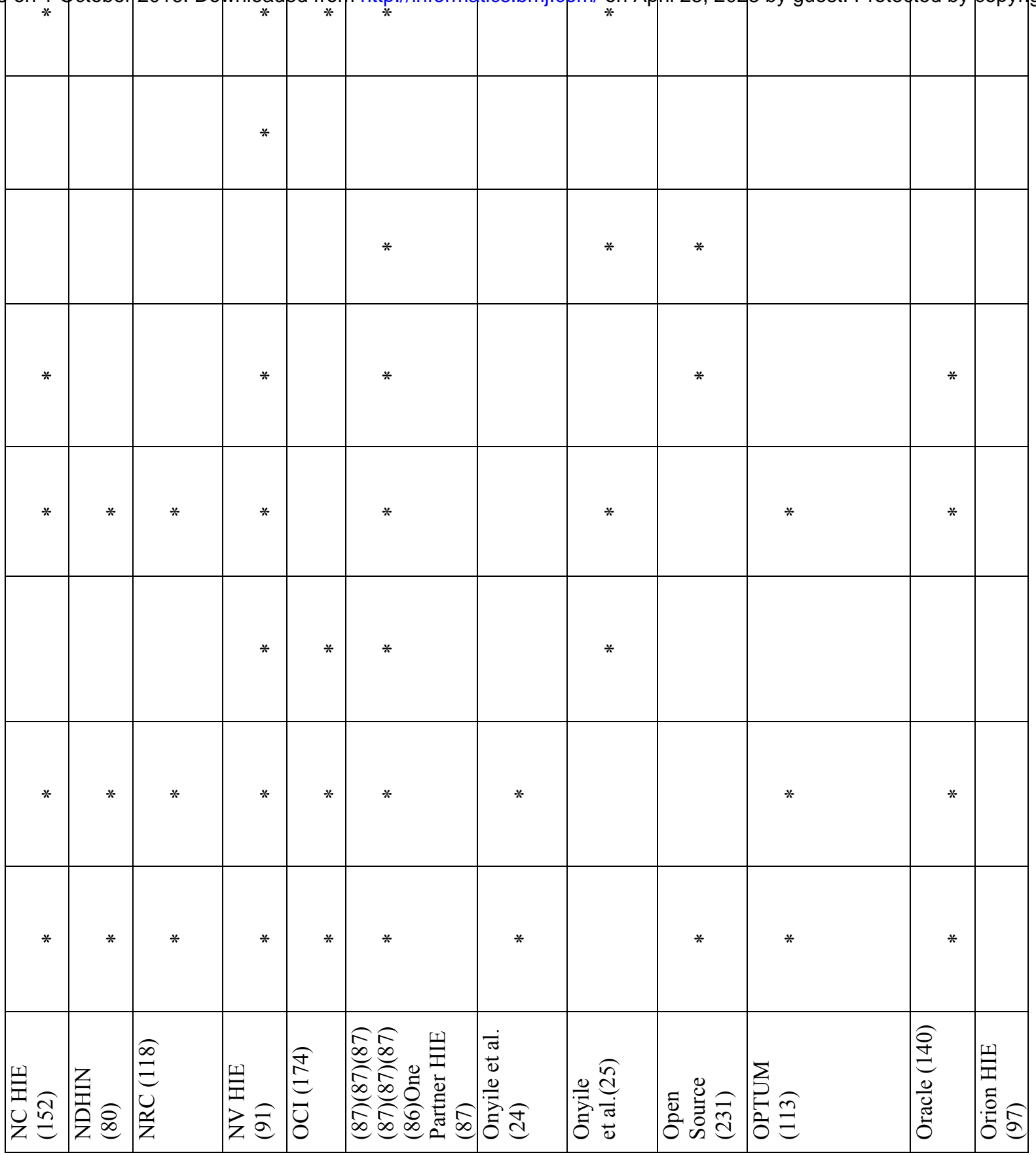


员

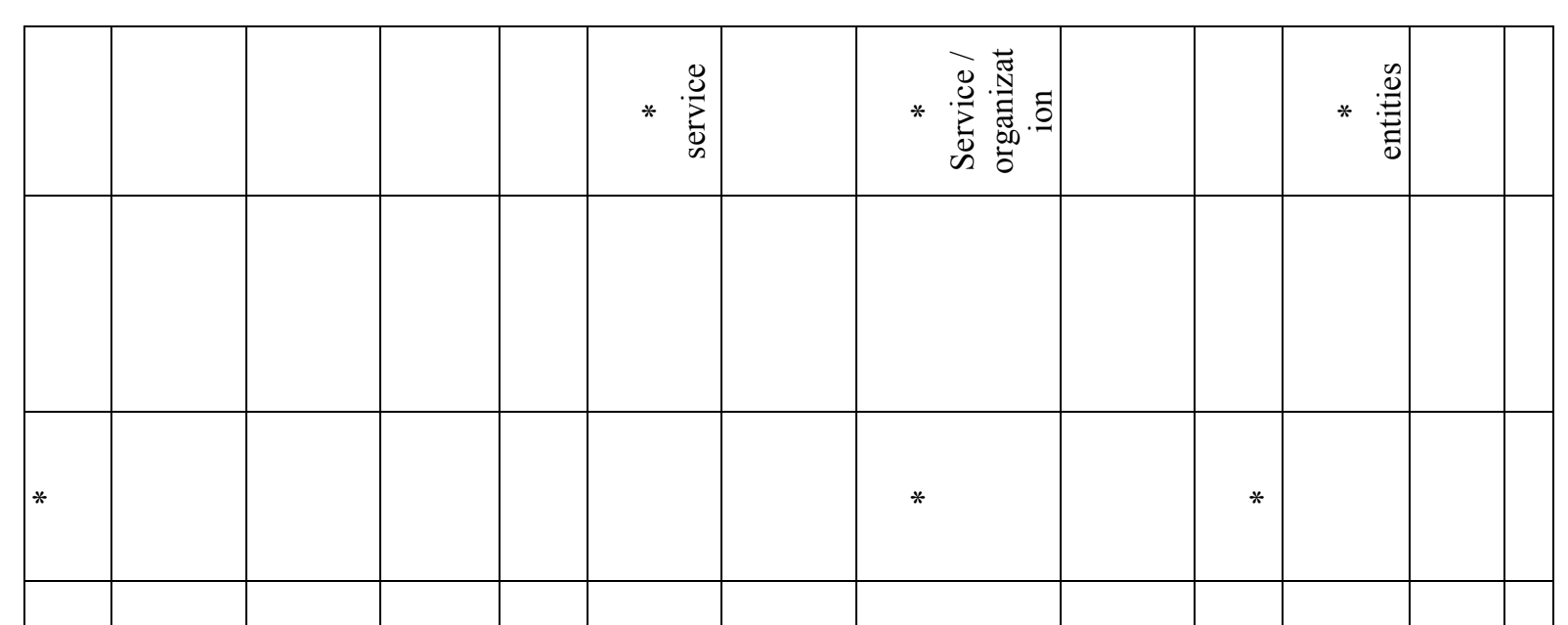

4236/jhi.v23i4.838 on 1 October 2016. Downloaded from http://informatics.bmj.com/ on April 25, 2023 by guest. Protected by copyright.

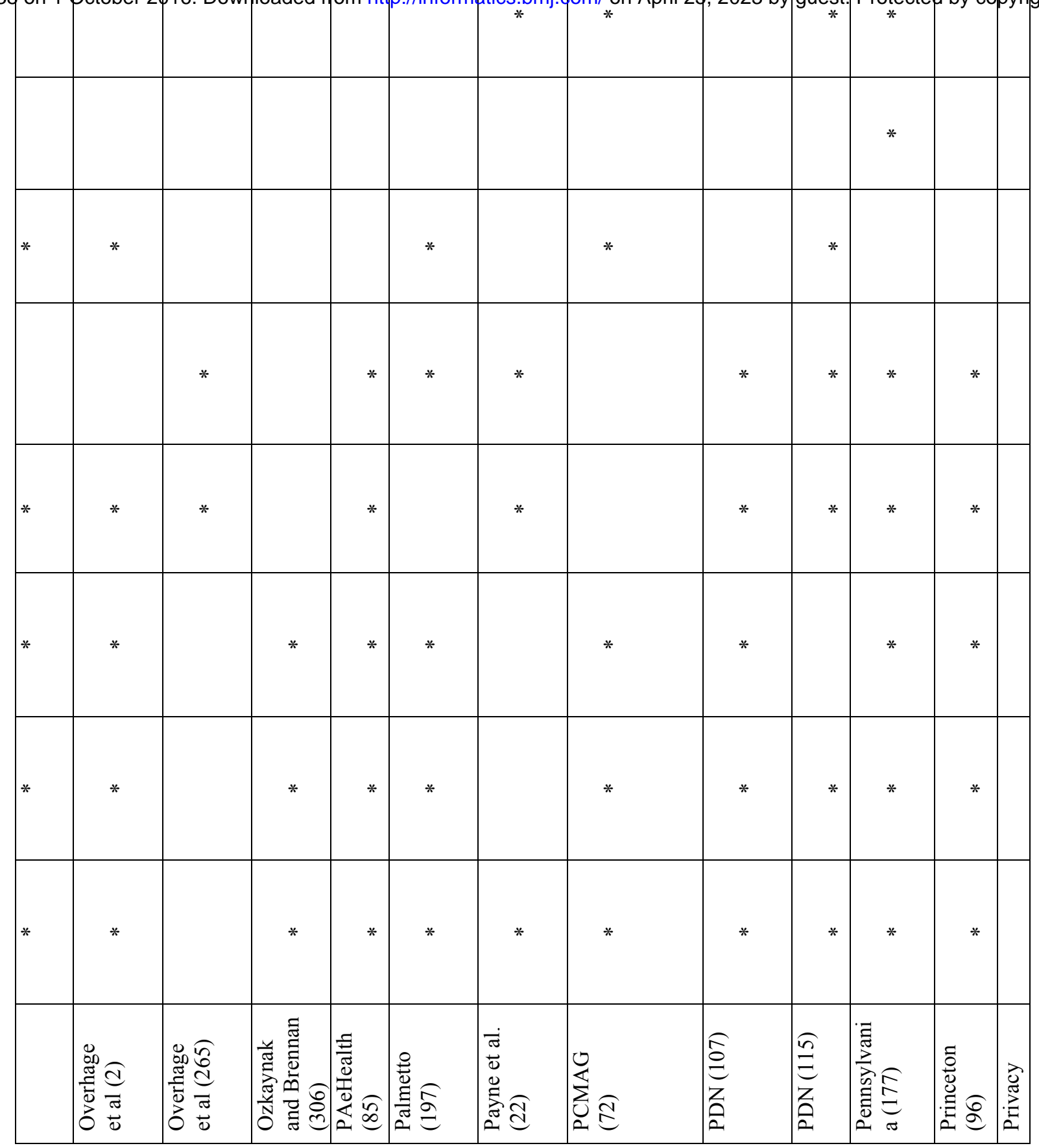



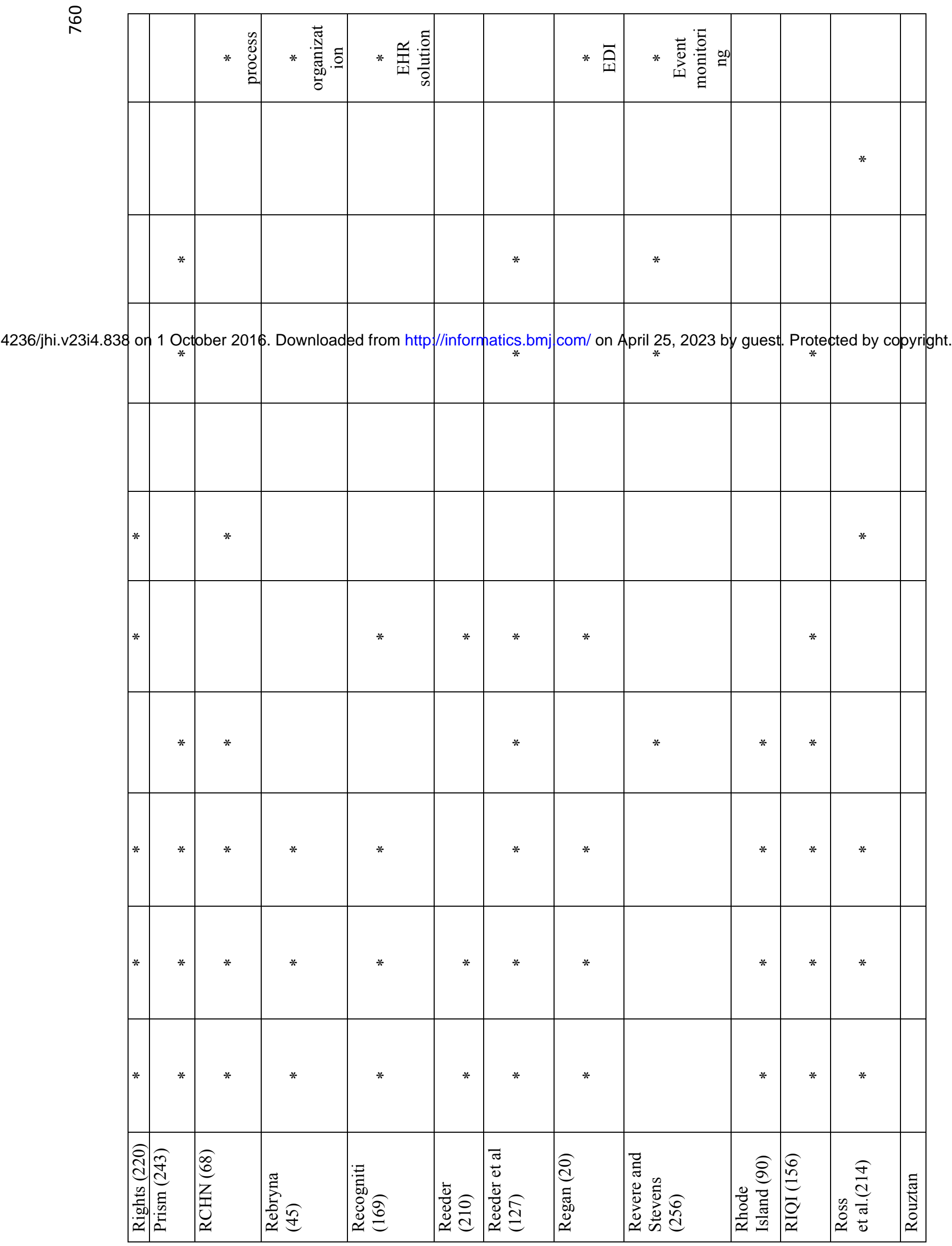


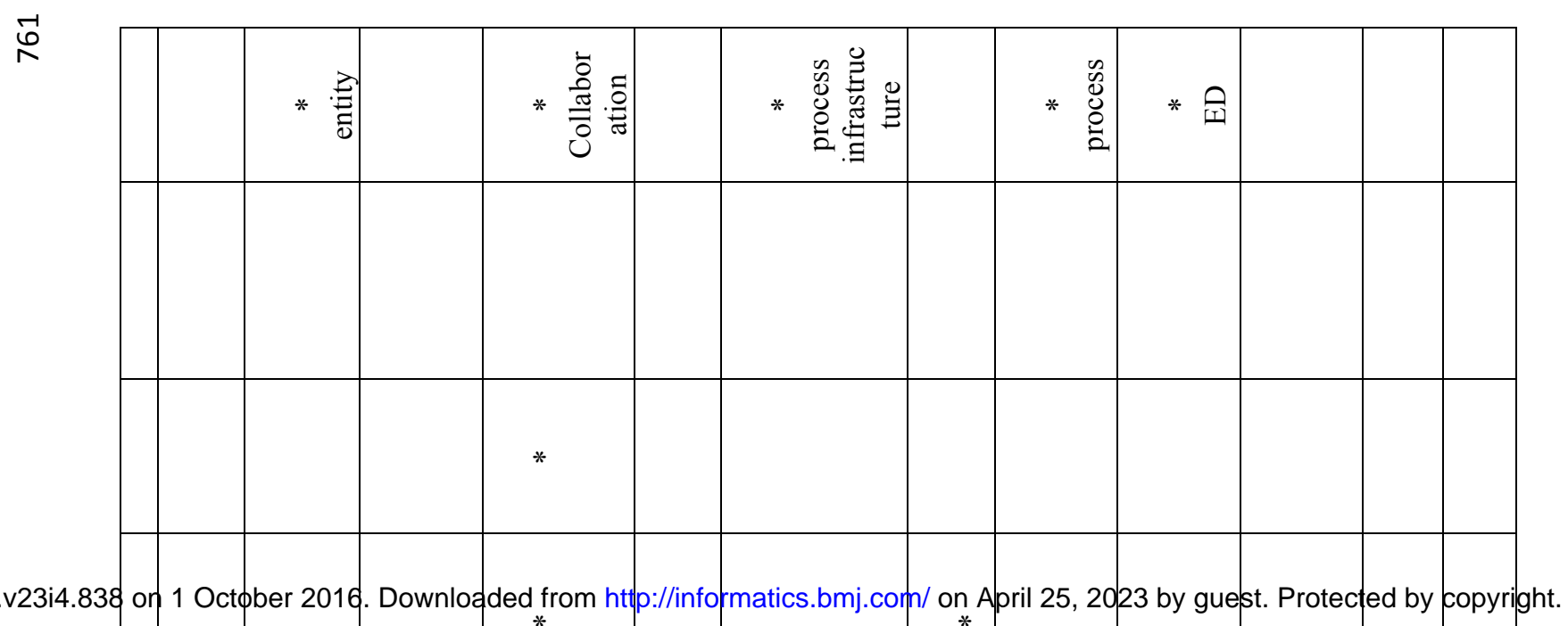

4236/jhi.v23i4.838 on 1 October 2016. Downloaded from http://informatics.bmj.com/ on April 25, 2023 by guest. Protected by copyright.

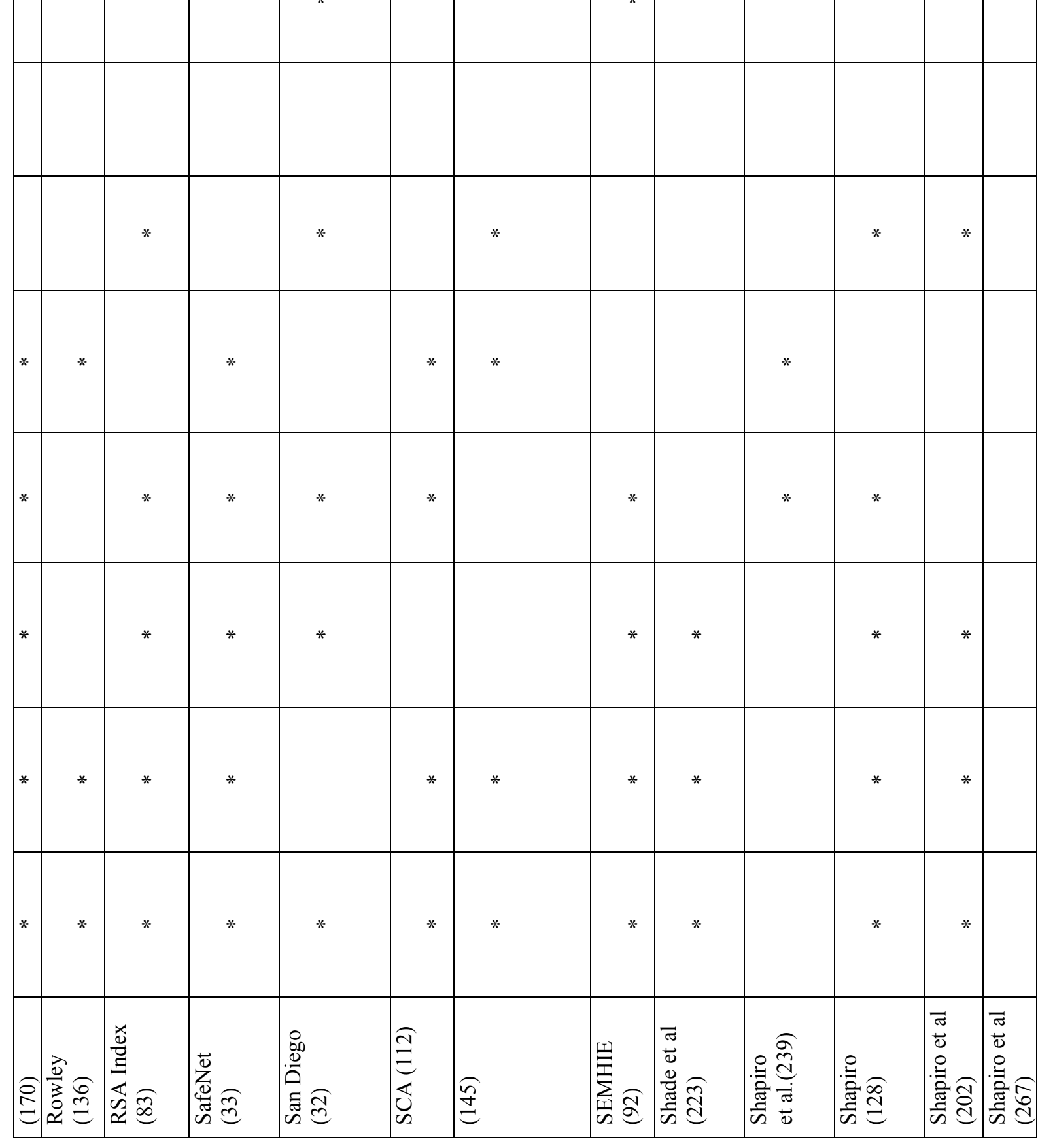


ำ

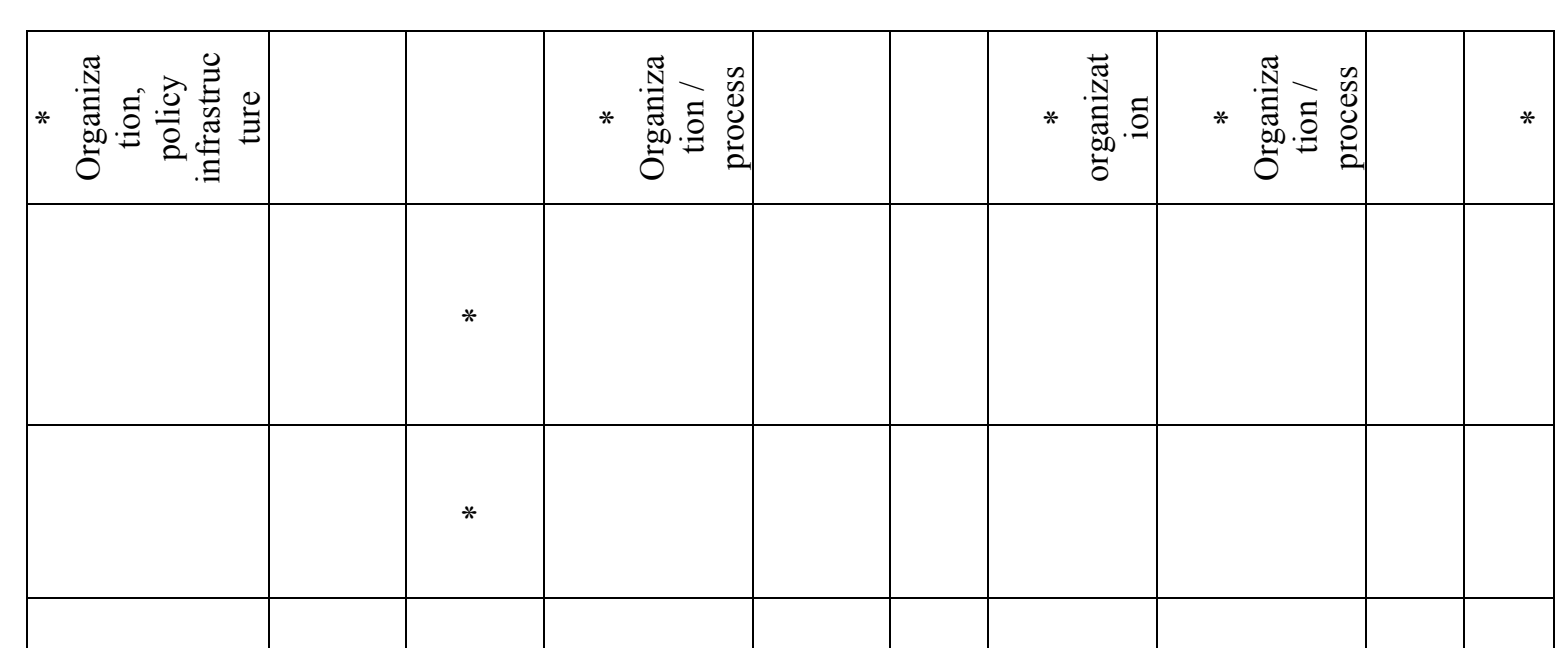

4236/jhi.v23i4.838 on 1 October 2016. Downlpaded from http://informatics.bmj.com/ on April 25, 2023 by guest. Protected by copyright.

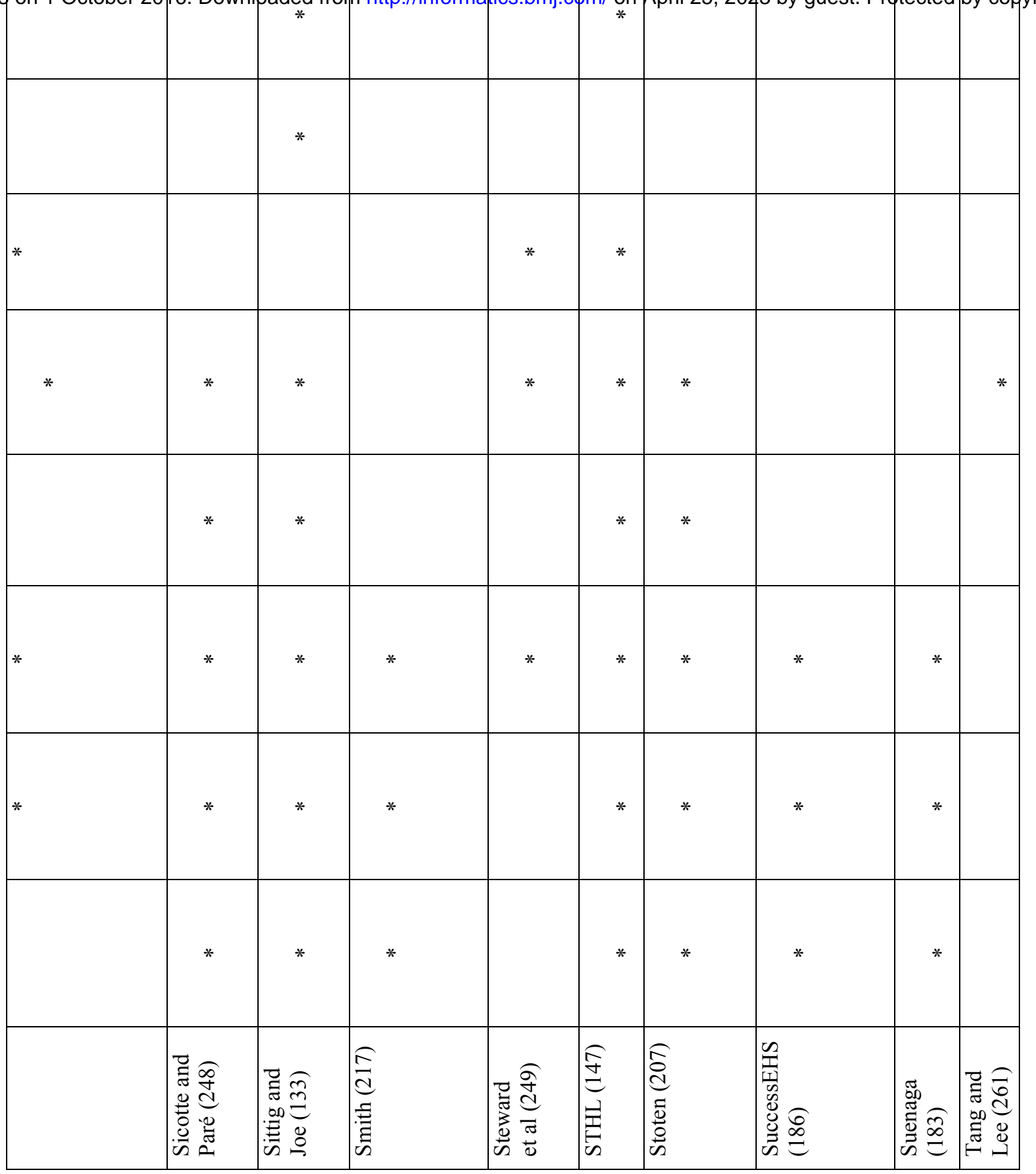


$\stackrel{m}{\circ}$

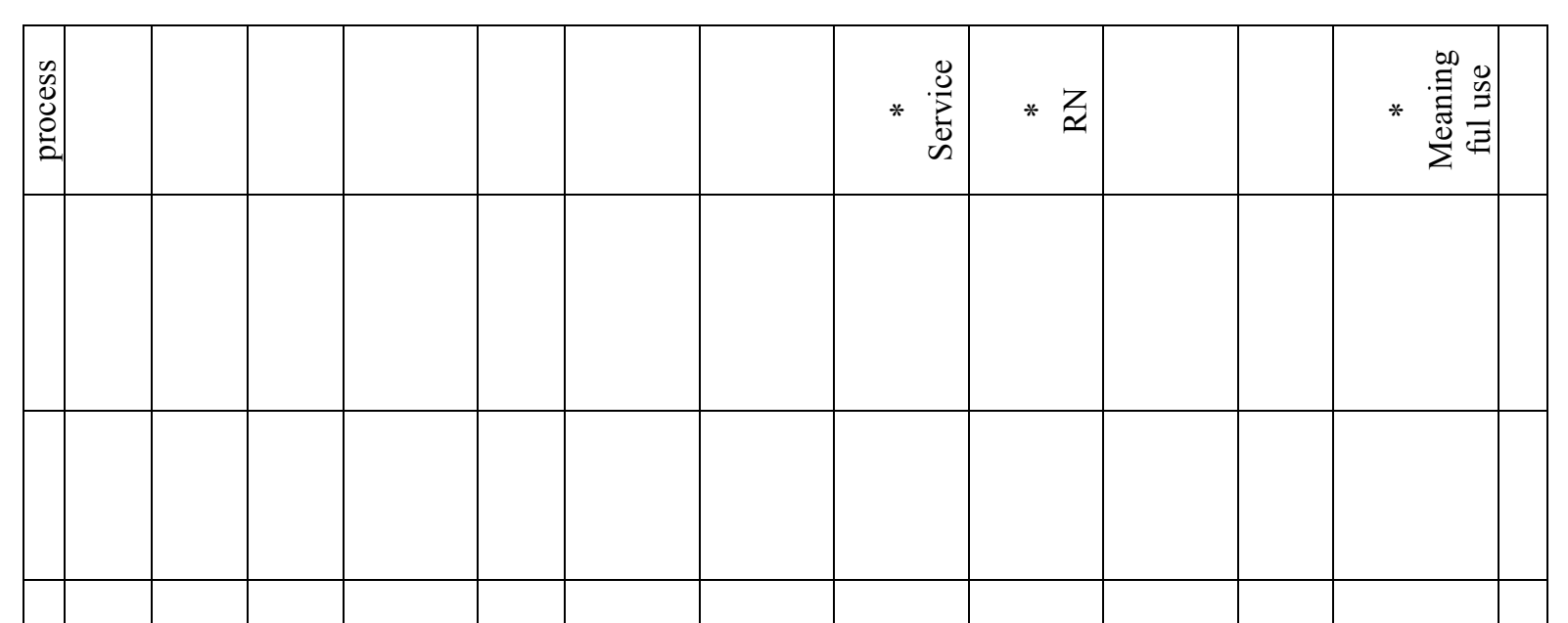

4236/jhi.v23i4.83@ on 1 October 2ф16. Downloaded from http://informatics.bm].com/ on April 25, 2 p23 by guest. Protected by copyright.

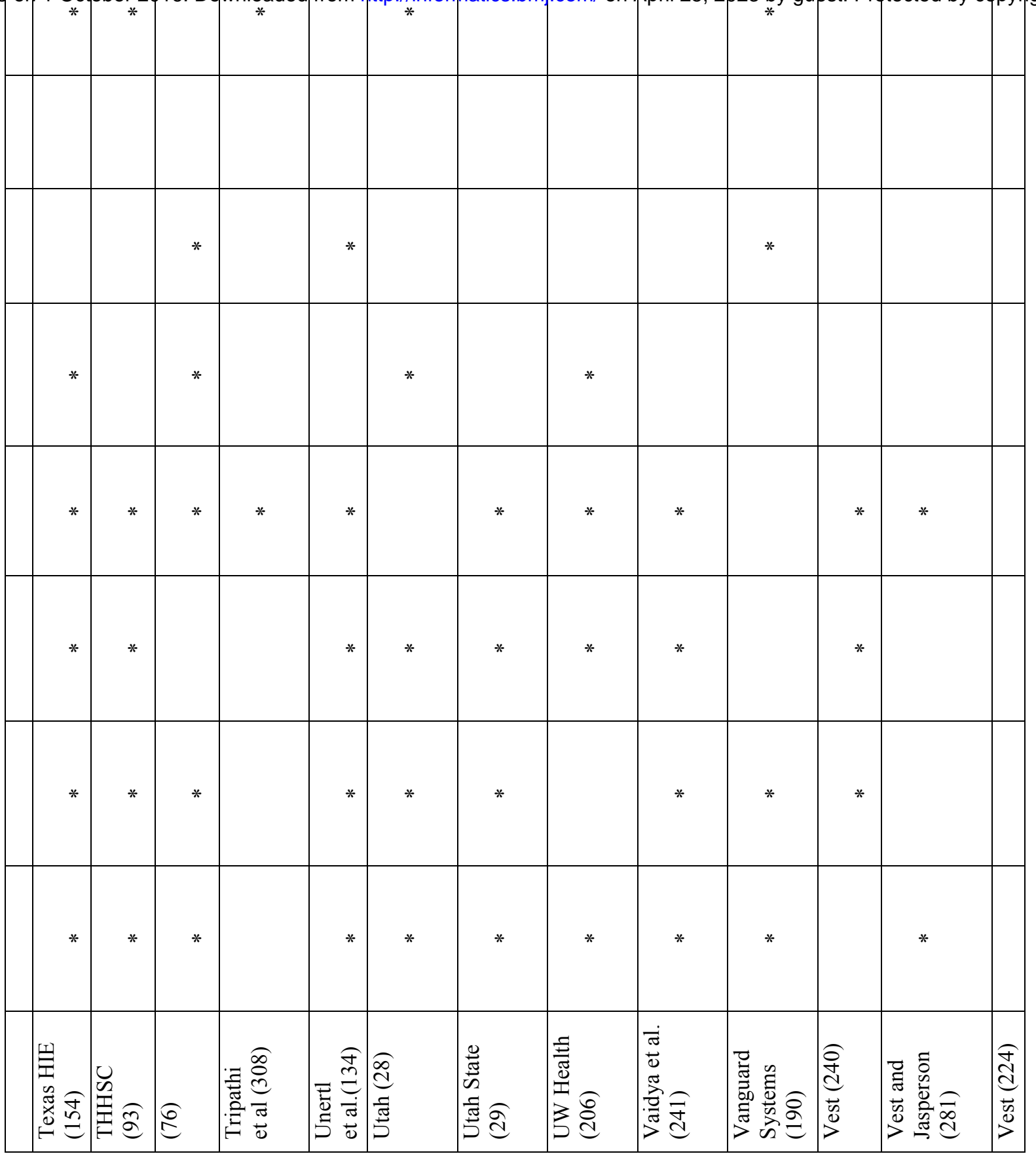


$\stackrel{\ddagger}{\circledR}$

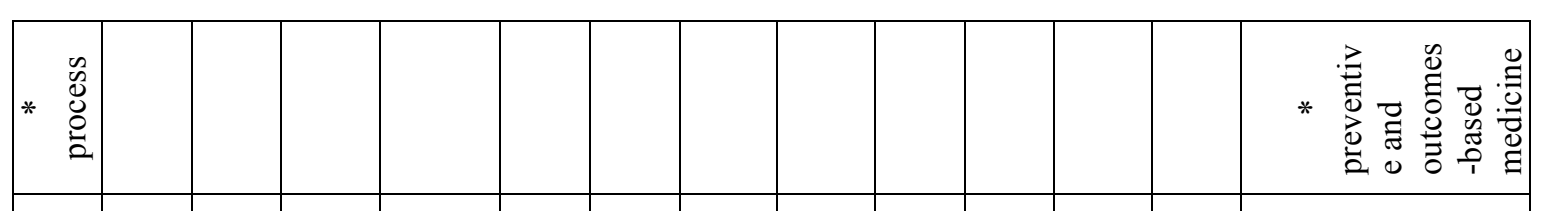

4236/jhi.v23i4.838 on 1 October 2016. Downloaded from http://informatic\$.bmj.com/ on April 25, $202 \beta$ by guest. Protected by copyright.

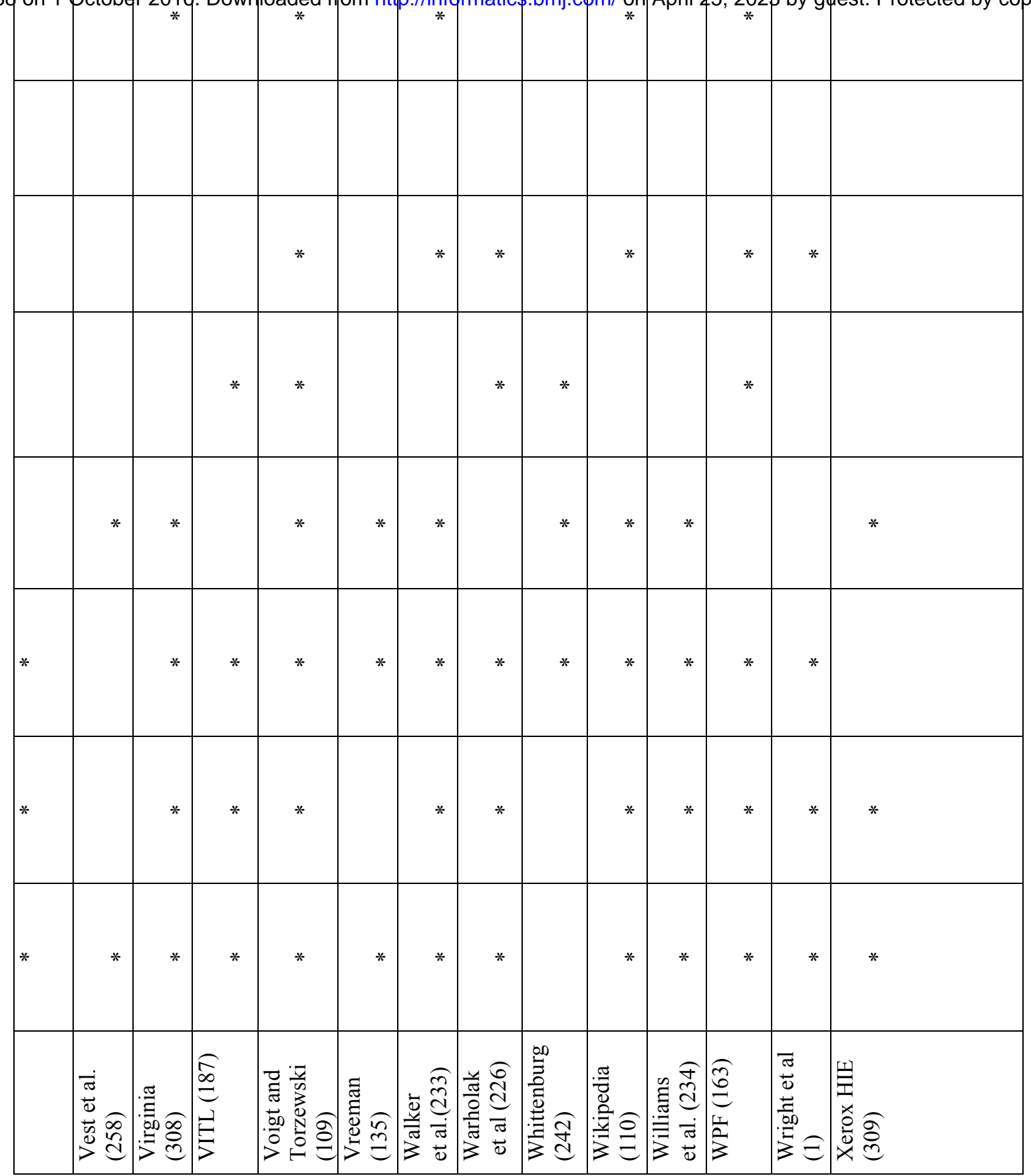

